\title{
Methylenespiro[2.3]hexanes via Nickel-Catalyzed Cyclopropanations with [1.1.1]Propellane
}

\author{
Songjie Yu, Adam Noble, Robin B. Bedford,* and Varinder K. Aggarwal* \\ School of Chemistry, University of Bristol, Cantock's Close, Bristol BS8 1TS, U.K.
}

\section{Contents}

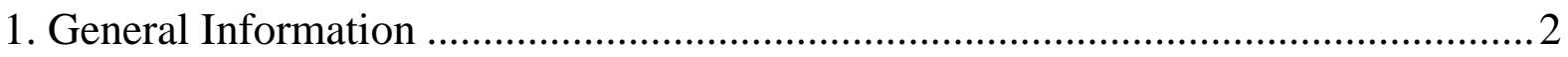

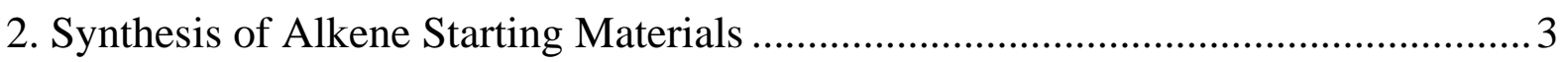

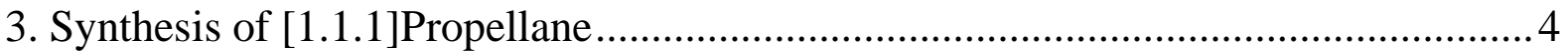

4. General Procedures and Product Characterization ....................................................5

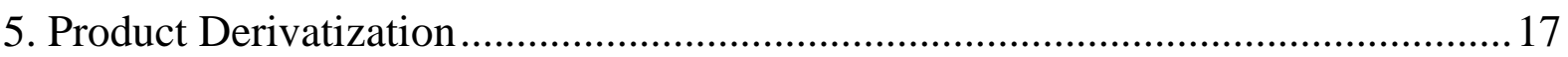

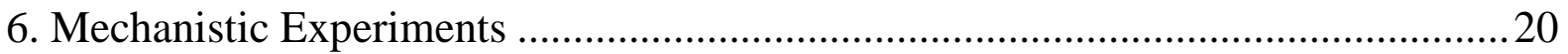

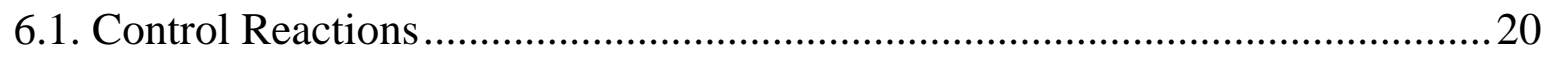

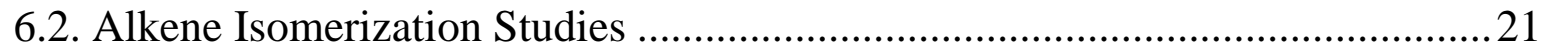

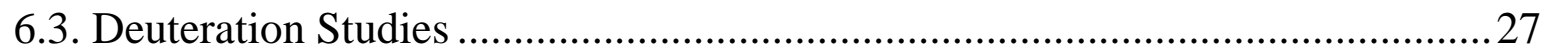

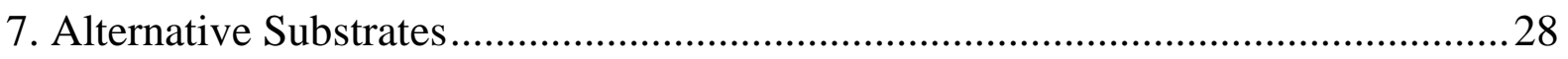

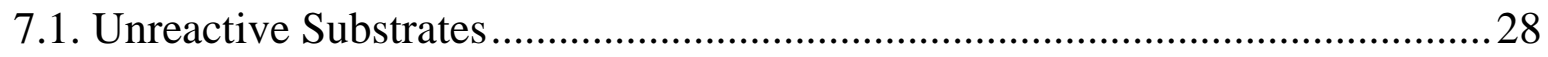

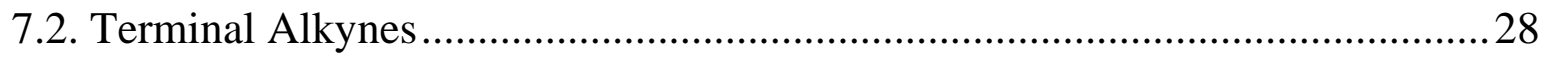

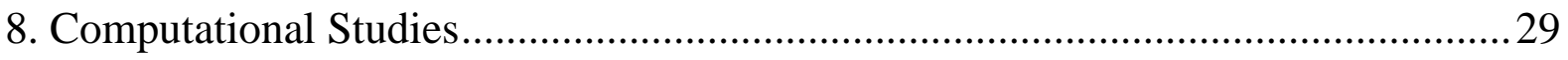

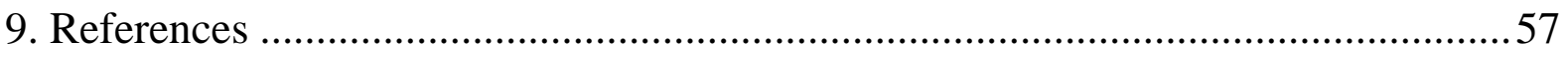

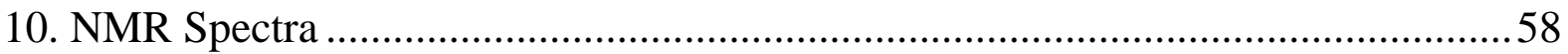




\section{General Information}

All dry solvents were commercially supplied or purified by passing the degassed solvents $\left(\mathrm{N}_{2}\right)$ through a column of activated alumina. All commercially available reagents were used as received unless otherwise stated. Styrene was filtered through silica gel prior to use. All reactions were carried out using Schlenk techniques or in an $\mathrm{N}_{2}$-filled glovebox. NMR Spectra $\left({ }^{1} \mathrm{H},{ }^{13} \mathrm{C},{ }^{19} \mathrm{~F}\right.$ and $\left.{ }^{11} \mathrm{~B}\right)$ were acquired on Jeol ECS $400 \mathrm{MHz}$ or Bruker $400 \mathrm{MHz}$ spectrometers in the solvents indicated. Chemical shifts $(\delta)$ are given in parts per million (ppm) and referenced to $\mathrm{CDCl}_{3}(7.26 \mathrm{ppm})$. High resolution mass spectra were obtained on Bruker Daltonics Apex IV by Electrospray Ionisation. IR spectra were recorded on a Perkin Elmer Spectrum One FT-IR as a thin film. Absorption maxima $\left(v_{\max }\right)$ are reported in wavenumbers $\left(\mathrm{cm}^{-1}\right)$. All reactions were followed by thin-layer chromatography (TLC) when practical, which were visualized under UV light or by staining with aqueous potassium permanganate, phosphomolybdic acid or $p$-anisaldehyde solutions. 


\section{Synthesis of Alkene Starting Materials}

$$
\mathrm{R} \curvearrowright \mathrm{O}+\mathrm{Ph}_{3} \mathrm{PMeBr} \underset{\mathrm{THF}}{\stackrel{\mathrm{LiO} \mathrm{Bu}^{\mathrm{B}}}{\longrightarrow}} \mathrm{R} \curvearrowright
$$

In a dry Schlenk flask, $\mathrm{Ph}_{3} \mathrm{PMeBr}(12 \mathrm{mmol})$ and $\mathrm{LiO}^{t} \mathrm{Bu}(15 \mathrm{mmol})$ were dissolved in THF $(20 \mathrm{~mL})$ and the mixture was stirred for $15 \mathrm{~min}$. Aldehyde $(10 \mathrm{mmol})$ was added and the mixture was stirred overnight at room temperature. Hexane $(50 \mathrm{~mL})$ was added and the mixture was filtered through celite. The filtrate was concentrated under reduced pressure and the residue was purified by silica gel flash column chromatography using pentane as eluent.

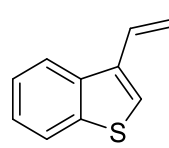

Colourless oil, 89\% yield. ${ }^{1} \mathrm{H}$ NMR (400 MHz, Chloroform- $d$ ) $\delta 7.94$ (dd, $J=7.6,1.3$ $\mathrm{Hz}, 1 \mathrm{H}), 7.91-7.84(\mathrm{~m}, 1 \mathrm{H}), 7.48(\mathrm{~s}, 1 \mathrm{H}), 7.40$ (dtd, $J=19.8,7.2,1.2 \mathrm{~Hz}, 2 \mathrm{H}), 7.00$ (ddd, $J=17.6,11.1,0.8 \mathrm{~Hz}, 1 \mathrm{H}), 5.83(\mathrm{dd}, J=17.6,1.3 \mathrm{~Hz}, 1 \mathrm{H}), 5.40(\mathrm{dd}, J=11.1,1.2$ $\mathrm{Hz}, 1 \mathrm{H}) .{ }^{13} \mathrm{C}$ NMR (100 MHz, Chloroform- $d$ ) $\delta 140.5,137.7,134.6,129.3,124.5,124.3$, $122.9,122.3,122.0,115.6$. All spectroscopic data are in agreement with literature reports. ${ }^{1}$

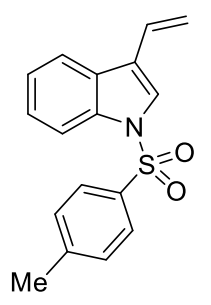

White solid, 93\% yield. ${ }^{1} \mathrm{H}$ NMR (400 MHz, Chloroform- $d$ ) $\delta 8.02(\mathrm{dt}, J=8.4,1.0 \mathrm{~Hz}$, $1 \mathrm{H}), 7.83-7.74(\mathrm{~m}, 3 \mathrm{H}), 7.63(\mathrm{~s}, 1 \mathrm{H}), 7.36(\mathrm{ddd}, J=8.4,7.3,1.3 \mathrm{~Hz}, 1 \mathrm{H}), 7.29(\mathrm{td}, J$ = 7.6, $1.2 \mathrm{~Hz}, 1 \mathrm{H}), 7.26-7.22(\mathrm{~m}, 2 \mathrm{H}), 6.79(\mathrm{ddd}, J=17.8,11.3,0.7 \mathrm{~Hz}, 1 \mathrm{H}), 5.82$ (dd, $J=17.8,1.2 \mathrm{~Hz}, 1 \mathrm{H}), 5.37(\mathrm{dd}, J=11.3,1.2 \mathrm{~Hz}, 1 \mathrm{H}), 2.36(\mathrm{~s}, 3 \mathrm{H}) .{ }^{13} \mathrm{C}$ NMR $(100$ MHz, Chloroform- $d$ ) $\delta$ 145.0, 135.5, 135.2, 129.9, 129.0, 127.6, 126.9, 124.9, 124.1, $123.5,121.0,120.4,115.3,113.7,21.6$. All spectroscopic data are in agreement with literature reports. ${ }^{2}$

Orange solid, 85\% yield. ${ }^{1} \mathrm{H}$ NMR (400 MHz, Chloroform- $d$ ) $\delta 6.48$ (dd, $J=17.5,10.7$ $\mathrm{Hz}, 1 \mathrm{H}), 5.37(\mathrm{dd}, J=17.5,1.6 \mathrm{~Hz}, 1 \mathrm{H}), 5.06(\mathrm{dd}, J=10.7,1.6 \mathrm{~Hz}, 1 \mathrm{H}), 4.39$ (t, $J=1.9$ $\mathrm{Hz}, 2 \mathrm{H}), 4.24$ (t, $J=1.9 \mathrm{~Hz}, 2 \mathrm{H}), 4.14(\mathrm{~s}, 5 \mathrm{H}) .{ }^{13} \mathrm{C}$ NMR $(100 \mathrm{MHz}$, Chloroform- $d$ ) $\delta$ 134.7, 111.1, 83.6, 69.3, 68.7, 66.7. All spectroscopic data are in agreement with literature reports. ${ }^{3}$

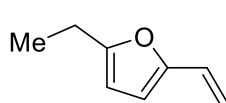

Colourless oil, 77\% yield. ${ }^{1} \mathrm{H}$ NMR (400 MHz, Chloroform- $d$ ) $\delta 6.46$ (dd, $J=17.5$, $11.3 \mathrm{~Hz}, 1 \mathrm{H}), 6.16(\mathrm{~d}, J=3.1 \mathrm{~Hz}, 1 \mathrm{H}), 5.98(\mathrm{~d}, J=3.0 \mathrm{~Hz}, 1 \mathrm{H}), 5.60(\mathrm{dd}, J=17.5$, $1.4 \mathrm{~Hz}, 1 \mathrm{H}), 5.08(\mathrm{dd}, J=11.2,1.4 \mathrm{~Hz}, 1 \mathrm{H}), 2.68(\mathrm{q}, J=7.6 \mathrm{~Hz}, 2 \mathrm{H}), 1.26(\mathrm{t}, J=7.6$ $\mathrm{Hz}, 3 \mathrm{H}) .{ }^{13} \mathrm{C}$ NMR (100 MHz, Chloroform- $d$ ) $\delta 157.8,151.7,125.3,110.6,109.0,105.8,21.6,12.2$.

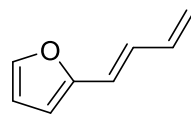

Yellow oil, $76 \%$ yield. ${ }^{1} \mathrm{H}$ NMR (400 MHz, Chloroform- $d$ ) $\delta 7.36(\mathrm{~d}, J=1.7 \mathrm{~Hz}, 1 \mathrm{H})$, $6.70(\mathrm{dd}, J=15.6,10.8 \mathrm{~Hz}, 1 \mathrm{H}), 6.50-6.33(\mathrm{~m}, 3 \mathrm{H}), 6.28(\mathrm{~d}, J=3.3 \mathrm{~Hz}, 1 \mathrm{H}), 5.39-$ $5.26(\mathrm{~m}, 1 \mathrm{H}), 5.16(\mathrm{~d}, J=10.1 \mathrm{~Hz}, 1 \mathrm{H}) .{ }^{13} \mathrm{C}$ NMR $(101 \mathrm{MHz}$, Chloroform- $d) \delta 153.0$, $142.2,136.7,128.2,120.4,117.8,111.6,108.5$. All spectroscopic data are in agreement with literature reports. ${ }^{4}$ 


\section{Synthesis of [1.1.1]Propellane}

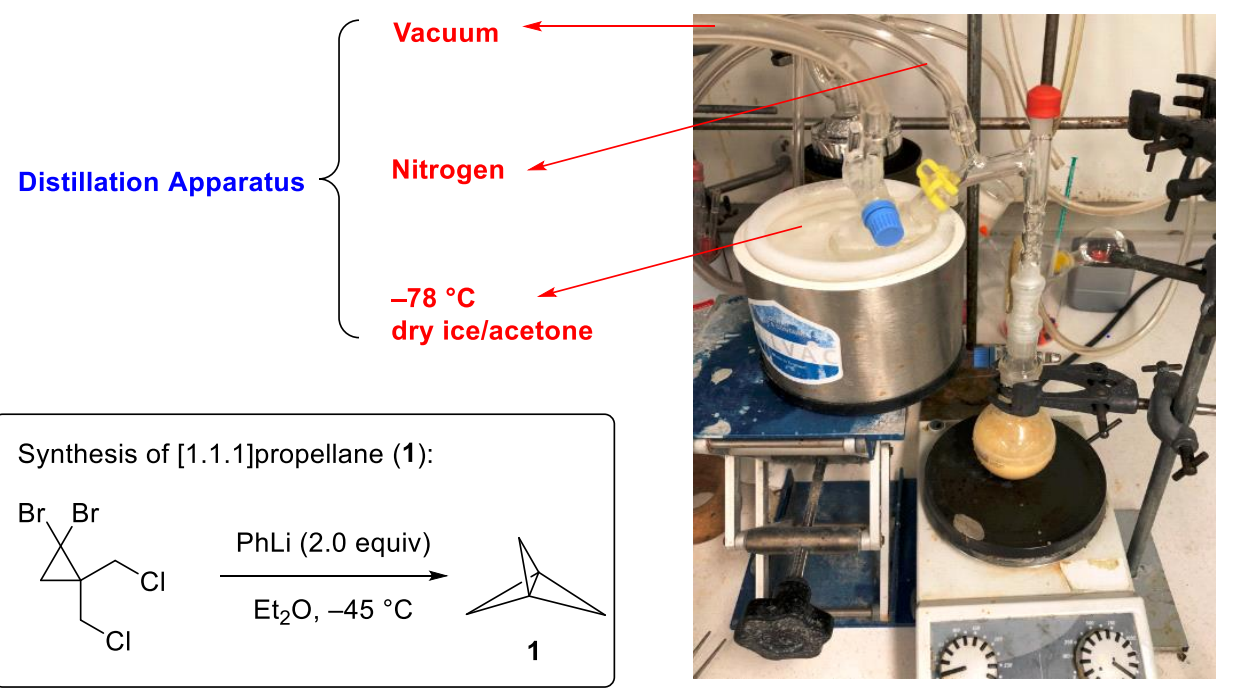

In a dry Schlenk flask 1,1-dibromo-2,2-bis(chloromethyl)cyclopropane (8.91 g, $30 \mathrm{mmol}$ ) was dissolved in $\mathrm{Et}_{2} \mathrm{O}(25 \mathrm{~mL})$ at $-45{ }^{\circ} \mathrm{C}$. Phenyllithium (1.9 M in dibutyl ether, $\left.31.5 \mathrm{~mL}, 60 \mathrm{mmol}\right)$ was added dropwise over $20 \mathrm{~min}$. After the addition was complete, the mixture was stirred at $-45^{\circ} \mathrm{C}$ for $10 \mathrm{~min}$ and then warmed to $0{ }^{\circ} \mathrm{C}$. After $2 \mathrm{~h}$ at this temperature, the [1.1.1]propellane solution was distilled under reduced pressure ( $\sim 40 \mathrm{mbar}$ ) at RT for $40 \mathrm{~min}$. A volume of approximately $25 \mathrm{~mL}$ of distillate was collected. An aliquot $(0.3 \mathrm{~mL})$ was analyzed by ${ }^{1} \mathrm{H} \mathrm{NMR}$ with $\mathrm{CH}_{2} \mathrm{Br}_{2}$ as an internal standard (see spectrum below). The average concentration of [1.1.1]propellane was 0.7-0.9 M. The solution was stored at $-25^{\circ} \mathrm{C}$ in a glovebox. 


\section{General Procedures and Product Characterization}

\section{General Procedure A:}

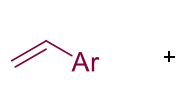

8

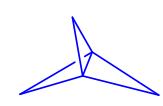

1
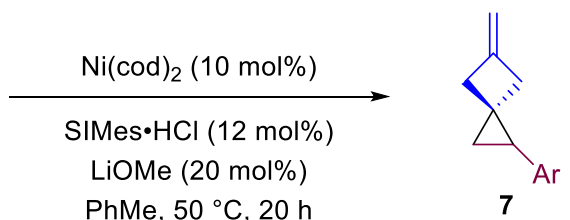

$\mathrm{Ni}(\operatorname{cod})_{2}(10 \mathrm{~mol} \%, 5.6 \mathrm{mg})$, SIMes $\cdot \mathrm{HCl}(12 \mathrm{~mol} \%, 8.3 \mathrm{mg})$ and LiOMe (20 mol\%, $\left.1.5 \mathrm{mg}\right)$ were dissolved in toluene $(1 \mathrm{~mL})$ in a pressure tube under an $\mathrm{N}_{2}$ atmosphere. The mixture was stirred for 15 $\mathrm{min}$ at RT before adding a solution of styrene-derivative $(0.20 \mathrm{mmol})$ and [1.1.1]propellane $(0.7-0.9$ $\mathrm{M}$ in $\left.\mathrm{Et}_{2} \mathrm{O}, 0.8 \mathrm{mmol}\right)$ in toluene $(2 \mathrm{~mL})$. The reaction mixture was heated to $50{ }^{\circ} \mathrm{C}$ and stirred for 20 h. After allowing to cool to RT, the solvent was removed under reduced pressure and the residue was purified by silica gel flash column chromatography using pentane as eluent.

\section{General Procedure B:}
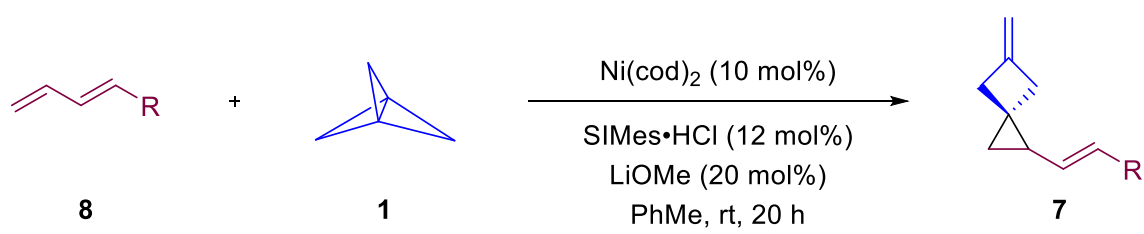

$\mathrm{Ni}(\operatorname{cod})_{2}(10 \mathrm{~mol} \%, 5.6 \mathrm{mg})$, SIMes $\cdot \mathrm{HCl}(12 \mathrm{~mol} \%, 8.3 \mathrm{mg})$ and LiOMe $(20 \mathrm{~mol} \%, 1.5 \mathrm{mg})$ were dissolved in toluene $(1 \mathrm{~mL})$ in a pressure tube under an $\mathrm{N}_{2}$ atmosphere. The mixture was stirred for 15 $\mathrm{min}$ at RT before adding a solution of diene/enyne $(0.20 \mathrm{mmol})$ and [1.1.1]propellane $(0.7-0.9 \mathrm{M}$ in $\left.\mathrm{Et}_{2} \mathrm{O}, 0.8 \mathrm{mmol}\right)$ in toluene $(2 \mathrm{~mL})$. The reaction mixture was stirred at $\mathrm{RT}$ for $20 \mathrm{~h}$ before concentrating under reduced pressure. The residue was purified by silica gel flash column chromatography using pentane as eluent.

\section{General Procedure C:}<smiles>[R]C=C([R])OC1(C)OC(C)(C)C1(C)C</smiles>

9
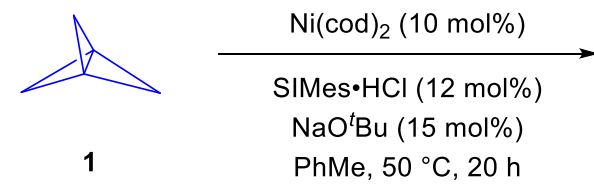

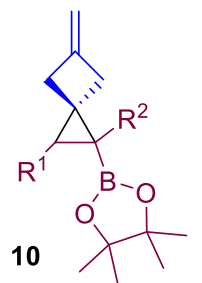

$\mathrm{Ni}(\operatorname{cod})_{2}(10 \mathrm{~mol} \%, 5.6 \mathrm{mg})$, SIMes $\cdot \mathrm{HCl}(12 \mathrm{~mol} \%, 8.3 \mathrm{mg})$ and $\mathrm{NaO}^{t} \mathrm{Bu}(15 \mathrm{~mol} \%, 2.9 \mathrm{mg})$ were dissolved in toluene $(1 \mathrm{~mL})$ in a pressure tube under an $\mathrm{N}_{2}$ atmosphere. The mixture was stirred for 15 $\mathrm{min}$ at RT before adding a solution of alkenylboronic ester $(0.20 \mathrm{mmol})$ and [1.1.1]propellane $(0.7-$ $0.9 \mathrm{M}$ in $\left.\mathrm{Et}_{2} \mathrm{O}, 0.8 \mathrm{mmol}\right)$ in toluene $(2 \mathrm{~mL})$. The reaction mixture was heated to $50{ }^{\circ} \mathrm{C}$ and stirred for $20 \mathrm{~h}$. After allowing to cool to RT, the solvent was removed under reduced pressure and the residue was purified by silica gel flash column chromatography using pentane as eluent. 


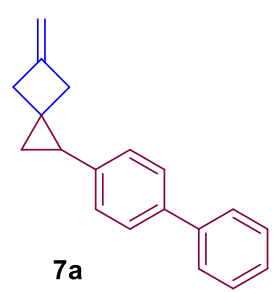

Prepared following General Procedure A, using Ni(cod) $)_{2}(10 \mathrm{~mol} \%, 5.6 \mathrm{mg})$, SIMes $\cdot \mathrm{HCl}(12 \mathrm{~mol} \%, 8.3 \mathrm{mg})$, LiOMe (20 mol\%, $1.5 \mathrm{mg})$, 4-vinylbiphenyl (0.20 $\mathrm{mmol}, 36 \mathrm{mg})$, and [1.1.1]propellane $(0.80 \mathrm{mmol})$ in toluene $(3 \mathrm{~mL})$. Purification by flash column chromatography with pentane as eluent $\left(\mathrm{R}_{\mathrm{f}}=0.3\right)$ gave $7 \mathbf{a}(43.8$ $\mathrm{mg}, 89 \%$ yield) as a colourless oil. IR (film) $v_{\max } / \mathrm{cm}^{-1}: 3057,3028,2987,2930$, 2902, 1676, 1612, 1488, 876, 762, 696. HRMS (APCI): Calcd. for $\mathrm{C}_{19} \mathrm{H}_{19}$ $\left([\mathrm{M}+\mathrm{H}]^{+}\right) \mathrm{m} / \mathrm{z}$ 247.1481, found $\mathrm{m} / \mathrm{z}$ 247.1477. ${ }^{1} \mathrm{H}$ NMR (400 MHz, Chloroform- $d$ ) $\delta 7.63-7.58(\mathrm{~m}, 2 \mathrm{H}), 7.53(\mathrm{~d}, J=8.1 \mathrm{~Hz}, 2 \mathrm{H}), 7.45(\mathrm{t}, J=7.7 \mathrm{~Hz}, 2 \mathrm{H}), 7.39-7.31(\mathrm{~m}, 1 \mathrm{H}), 7.07(\mathrm{~d}$, $J=8.1 \mathrm{~Hz}, 2 \mathrm{H}), 4.87(\mathrm{p}, J=2.5 \mathrm{~Hz}, 1 \mathrm{H}), 4.85(\mathrm{p}, J=2.5 \mathrm{~Hz}, 1 \mathrm{H}), 2.98(\mathrm{dq}, J=15.8,2.8 \mathrm{~Hz}, 1 \mathrm{H})$, $2.90(\mathrm{dq}, J=15.8,2.8 \mathrm{~Hz}, 1 \mathrm{H}), 2.79$ (dq, $J=16.0,2.7 \mathrm{~Hz}, 1 \mathrm{H}), 2.69$ (dq, $J=16.0,2.9 \mathrm{~Hz}, 1 \mathrm{H}), 2.05$ $(\mathrm{dd}, J=9.0,5.9 \mathrm{~Hz}, 1 \mathrm{H}), 1.24(\mathrm{dd}, J=8.9,5.4 \mathrm{~Hz}, 1 \mathrm{H}), 1.03(\mathrm{t}, J=5.7 \mathrm{~Hz}, 1 \mathrm{H}) .{ }^{13} \mathrm{C} \mathrm{NMR}(100 \mathrm{MHz}$, Chloroform- $d$ ) $\delta 144.5,141.1,140.2,138.2,128.7,127.04,126.97,126.9,126.8,106.4,40.7,36.2$, 27.5, 24.8, 19.9.

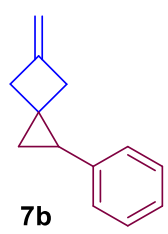

Prepared following General Procedure A, using Ni(cod $)_{2}(10 \mathrm{~mol} \%, 5.6 \mathrm{mg})$, SIMes $\cdot \mathrm{HCl}$ (12 mol\%, $8.3 \mathrm{mg})$, LiOMe (20 mol\%, $1.5 \mathrm{mg})$, styrene $(0.20 \mathrm{mmol}, 21 \mathrm{mg})$, and [1.1.1]propellane $(0.80 \mathrm{mmol})$ in toluene $(3 \mathrm{~mL})$. Purification by flash column chromatography with pentane as eluent gave $7 \mathbf{b}(27.5 \mathrm{mg}, 81 \%$ yield $)$ as a colourless oil. IR (film) $v_{\max } / \mathrm{cm}^{-1}: 3063,2988,2903,1677,1604,1498,876,769,697$. HRMS (APCI): Calcd. for $\mathrm{C}_{13} \mathrm{H}_{15}\left([\mathrm{M}+\mathrm{H}]^{+}\right) \mathrm{m} / \mathrm{z}$ 171.1168, found $\mathrm{m} / \mathrm{z}$ 171.1165. ${ }^{1} \mathrm{H}$ NMR (400 MHz, Chloroform- $d$ ) $\delta$ 7.30-7.26 (m, 2H), $7.18-7.12(\mathrm{~m}, 1 \mathrm{H}), 7.00-6.94(\mathrm{~m}, 2 \mathrm{H}), 4.83(\mathrm{p}, J=2.5 \mathrm{~Hz}, 1 \mathrm{H})$, $4.80(\mathrm{p}, J=2.5 \mathrm{~Hz}, 1 \mathrm{H}), 2.93(\mathrm{dq}, J=16.0,2.7 \mathrm{~Hz}, 1 \mathrm{H}), 2.85(\mathrm{dq}, J=15.9,2.7 \mathrm{~Hz}, 1 \mathrm{H}), 2.72(\mathrm{dq}, J=$ $16.1,2.7 \mathrm{~Hz}, 1 \mathrm{H}), 2.59(\mathrm{dq}, J=16.1,2.9 \mathrm{~Hz}, 1 \mathrm{H}), 1.98(\mathrm{dd}, J=8.9,5.9 \mathrm{~Hz}, 1 \mathrm{H}), 1.17(\mathrm{dd}, J=9.0,5.4$ $\mathrm{Hz}, 1 \mathrm{H}), 0.97(\mathrm{t}, J=5.7 \mathrm{~Hz}, 1 \mathrm{H}) .{ }^{13} \mathrm{C}$ NMR $(100 \mathrm{MHz}$, Chloroform- $d$ ) $\delta 144.6,141.0,128.2,126.7$, 125.4, 106.4, 40.7, 36.2, 27.8, 24.7, 19.7 .

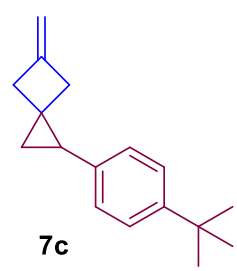

Prepared following General Procedure A, using $\mathrm{Ni}(\mathrm{cod})_{2}(10 \mathrm{~mol} \%$, $5.6 \mathrm{mg})$, SIMes $\cdot \mathrm{HCl}(12 \mathrm{~mol} \%, 8.3 \mathrm{mg})$, LiOMe $(20 \mathrm{~mol} \%$, $1.5 \mathrm{mg})$, 1-(tert-butyl)-4vinylbenzene $(0.20 \mathrm{mmol}, 32 \mathrm{mg})$, and [1.1.1]propellane $(0.80 \mathrm{mmol})$ in toluene $(3$ $\mathrm{mL}$ ). Purification by flash column chromatography with pentane as eluent gave 7c (33.0 mg, 73\% yield) as a colourless oil. IR (film) $v_{\max } / \mathrm{cm}^{-1}: 2962,2904,1677,1515$, 875, 826. HRMS (APCI): Calcd. for $\mathrm{C}_{17} \mathrm{H}_{23}\left([\mathrm{M}+\mathrm{H}]^{+}\right) \mathrm{m} / \mathrm{z}$ 227.1794, found $\mathrm{m} / \mathrm{z}$ 227.1792. ${ }^{1} \mathrm{H}$ NMR (400 MHz, Chloroform- $d$ ) $\delta 7.30(\mathrm{~d}, J=8.1 \mathrm{~Hz}, 2 \mathrm{H}), 6.91(\mathrm{~d}, J=8.0 \mathrm{~Hz}, 2 \mathrm{H})$, $4.86-4.81(\mathrm{~m}, 1 \mathrm{H}), 4.81-4.78(\mathrm{~m}, 1 \mathrm{H}), 2.92(\mathrm{dq}, J=15.8,2.4 \mathrm{~Hz}, 1 \mathrm{H}), 2.84(\mathrm{dq}, J=15.8,2.5 \mathrm{~Hz}$, $1 \mathrm{H}), 2.73(\mathrm{dq}, J=16.1,2.5 \mathrm{~Hz}, 1 \mathrm{H}), 2.65(\mathrm{dq}, J=16.1,2.5 \mathrm{~Hz}, 1 \mathrm{H}), 1.96(\mathrm{dd}, J=8.9,6.0 \mathrm{~Hz}, 1 \mathrm{H})$, $1.31(\mathrm{~s}, 9 \mathrm{H}), 1.16(\mathrm{dd}, J=8.8,5.5 \mathrm{~Hz}, 1 \mathrm{H}), 0.93(\mathrm{t}, J=5.6 \mathrm{~Hz}, 1 \mathrm{H}) .{ }^{13} \mathrm{C}$ NMR $(100 \mathrm{MHz}$, Chloroform- $d$ ) $\delta 148.1,144.8,138.0,126.3,125.1,106.3,40.7,36.3,34.4,31.5,27.4,24.4,19.9$.

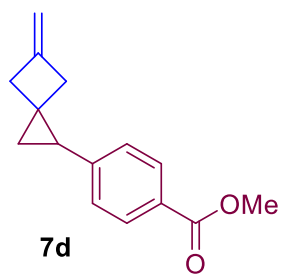

Prepared following General Procedure A, using Ni(cod) $)_{2}(10 \mathrm{~mol} \%, 5.6 \mathrm{mg})$, SIMes $\cdot \mathrm{HCl}$ (12 mol\%, $8.3 \mathrm{mg}$ ), LiOMe (20 mol\%, $1.5 \mathrm{mg})$, methyl 4vinylbenzoate $(0.20 \mathrm{mmol}, 33 \mathrm{mg})$, and [1.1.1]propellane $(0.80 \mathrm{mmol})$ in toluene ( $3 \mathrm{~mL}$ ). Purification by flash column chromatography with pentane/diethyl ether $(60 / 1)$ as eluent gave $\mathbf{7 d}(36.1 \mathrm{mg}, 79 \%$ yield) as a colourless oil. IR (film) $v_{\max } / \mathrm{cm}^{-1}$ : 3068, 2992, 2951, 2902, 1717, 1609, 1275, 1180, 1107, 777, 705. HRMS (ESI): Calcd. for $\mathrm{C}_{15} \mathrm{H}_{17} \mathrm{O}_{2}\left([\mathrm{M}+\mathrm{H}]^{+}\right) \mathrm{m} / \mathrm{z} 229.1223$, found $\mathrm{m} / \mathrm{z} 229.1224$. ${ }^{1} \mathrm{H}$ NMR (400 MHz, Chloroform- $d$ ) $\delta 7.96-7.90$ (m, 2H), $7.03-6.97$ (m, 2H), $4.83(\mathrm{p}, J=2.6 \mathrm{~Hz}$, $1 \mathrm{H}), 4.80(\mathrm{p}, J=2.4 \mathrm{~Hz}, 1 \mathrm{H}), 3.89(\mathrm{~s}, 3 \mathrm{H}), 2.95(\mathrm{dq}, J=16.0,2.6 \mathrm{~Hz}, 1 \mathrm{H}), 2.86(\mathrm{dq}, J=16.0,2.6 \mathrm{~Hz}$, 
$1 \mathrm{H}), 2.75(\mathrm{dq}, J=16.2,2.6 \mathrm{~Hz}, 1 \mathrm{H}), 2.56(\mathrm{dq}, J=16.1,2.8 \mathrm{~Hz}, 1 \mathrm{H}), 2.03(\mathrm{dd}, J=8.8,5.9 \mathrm{~Hz}, 1 \mathrm{H})$, $1.26(\mathrm{dd}, J=8.8,5.5 \mathrm{~Hz}, 1 \mathrm{H}), 1.05(\mathrm{t}, J=5.7 \mathrm{~Hz}, 1 \mathrm{H}) .{ }^{13} \mathrm{C}$ NMR $(100 \mathrm{MHz}$, Chloroform- $d) \delta 167.3$, 147.0, 144.0, 129.5, 127.2, 126.4, 106.7, 52.0, 40.7, 36.1, 28.1, 25.9, 20.7.

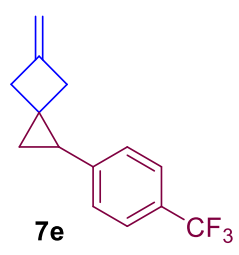

Prepared following General Procedure A, using Ni(cod) $)_{2}(10 \mathrm{~mol} \%, 5.6 \mathrm{mg})$, SIMes $\cdot \mathrm{HCl}$ (12 mol\%, $8.3 \mathrm{mg}$ ), LiOMe (20 mol\%, $1.5 \mathrm{mg}$ ), 1-(trifluoromethyl)-4vinylbenzene $(0.20 \mathrm{mmol}, 35 \mathrm{mg})$, and [1.1.1]propellane $(0.80 \mathrm{mmol})$ in toluene $(3$ $\mathrm{mL})$. Purification by flash column chromatography with pentane as eluent gave 7e (29 mg, 61\% yield) as a colourless oil. IR (film) $v_{\max } / \mathrm{cm}^{-1}: 3071,2989,2934,2906$, 1679, 1619, 1324, 1163, 1114, 1069, 879, 832. HRMS (APCI): Calcd. for $\mathrm{C}_{14} \mathrm{H}_{14} \mathrm{~F}_{3}$ $\left([\mathrm{M}+\mathrm{H}]^{+}\right) \mathrm{m} / \mathrm{z}$ 239.1042, found m/z 239.1038. ${ }^{1} \mathrm{H}$ NMR (400 MHz, Chloroform- $d$ ) $\delta 7.51(\mathrm{~d}, J=8.2$ $\mathrm{Hz}, 2 \mathrm{H}), 7.05(\mathrm{~d}, J=8.0 \mathrm{~Hz}, 2 \mathrm{H}), 4.85(\mathrm{p}, J=2.3 \mathrm{~Hz}, 1 \mathrm{H}), 4.82(\mathrm{p}, J=2.3 \mathrm{~Hz}, 1 \mathrm{H}), 3.00-2.91(\mathrm{~m}$, $1 \mathrm{H}), 2.91-2.84(\mathrm{~m}, 1 \mathrm{H}), 2.80-2.70(\mathrm{~m}, 1 \mathrm{H}), 2.61-2.51(\mathrm{~m}, 1 \mathrm{H}), 2.04(\mathrm{dd}, J=8.8,5.9 \mathrm{~Hz}, 1 \mathrm{H})$, $1.27(\mathrm{dd}, J=8.8,5.6 \mathrm{~Hz}, 1 \mathrm{H}), 1.03(\mathrm{t}, J=5.7 \mathrm{~Hz}, 1 \mathrm{H}) .{ }^{19} \mathrm{~F}$ NMR $(376 \mathrm{MHz}$, Chloroform- $d) \delta-62.1$. ${ }^{13} \mathrm{C}$ NMR (100 MHz, Chloroform- $d$ ) $\delta 145.5$ (q, $J=1.3 \mathrm{~Hz}$ ), 143.9, $127.6(\mathrm{q}, J=32.6 \mathrm{~Hz}), 126.8$, $125.0(\mathrm{q}, J=3.8 \mathrm{~Hz}), 124.5(\mathrm{q}, J=270.0 \mathrm{~Hz}), 106.8,40.6,36.1,27.7,25.6,20.4$.

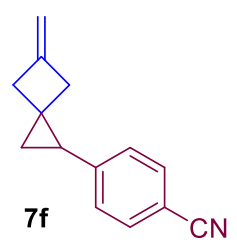

Prepared following General Procedure A, using Ni(cod) $)_{2}(10 \mathrm{~mol} \%$, $5.6 \mathrm{mg})$, SIMes $\cdot \mathrm{HCl}(12 \mathrm{~mol} \%, 8.3 \mathrm{mg})$, LiOMe (20 mol\%, $1.5 \mathrm{mg})$, 4-vinylbenzonitrile $(0.20$ $\mathrm{mmol}, 26 \mathrm{mg})$, and [1.1.1]propellane $(0.80 \mathrm{mmol})$ in toluene $(3 \mathrm{~mL})$. Purification by flash column chromatography with pentane/diethyl ether (30/1) as eluent gave 7f (33.9 mg, 87\% yield) as a colourless oil. IR (film) $v_{\max } / \mathrm{cm}^{-1}: 3072,2992,2934,2905$, 2225, 1677, 1607, 1508, 1178, 879, 830. HRMS (APCI): Calcd. for $\mathrm{C}_{14} \mathrm{H}_{14} \mathrm{~N}$ $\left([\mathrm{M}+\mathrm{H}]^{+}\right) \mathrm{m} / \mathrm{z}$ 196.1121, found $\mathrm{m} / \mathrm{z}$ 196.1120. ${ }^{1} \mathrm{H}$ NMR (400 MHz, Chloroform- $d$ ) $\delta 7.54(\mathrm{~d}, J=8.4$ $\mathrm{Hz}, 2 \mathrm{H}), 7.02(\mathrm{~d}, J=8.3 \mathrm{~Hz}, 2 \mathrm{H}), 4.84(\mathrm{p}, J=2.4 \mathrm{~Hz}, 1 \mathrm{H}), 4.82$ (p, $J=2.4 \mathrm{~Hz}, 1 \mathrm{H}), 3.00-2.91(\mathrm{~m}$, $1 \mathrm{H}), 2.91-2.84(\mathrm{~m}, 1 \mathrm{H}), 2.81-2.71(\mathrm{~m}, 1 \mathrm{H}), 2.60-2.50(\mathrm{~m}, 1 \mathrm{H}), 2.02(\mathrm{dd}, J=8.8,5.9 \mathrm{~Hz}, 1 \mathrm{H})$, $1.31(\mathrm{dd}, J=8.8,5.7 \mathrm{~Hz}, 1 \mathrm{H}), 1.04(\mathrm{t}, J=5.7 \mathrm{~Hz}, 1 \mathrm{H}) .{ }^{13} \mathrm{C}$ NMR $(100 \mathrm{MHz}$, Chloroform- $d$ ) $\delta 147.3$, 143.4, 132.0, 127.1, 119.4, 108.8, 107.0, 40.7, 36.0, 28.2, 26.4, 21.1.

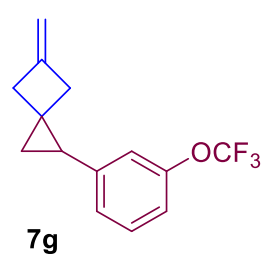

Prepared following General Procedure A, using Ni(cod) 2 (10 mol\%, $5.6 \mathrm{mg})$, SIMes $\cdot \mathrm{HCl}(12 \mathrm{~mol} \%, 8.3 \mathrm{mg})$, LiOMe (20 mol\%, $1.5 \mathrm{mg}), 1$-(trifluoromethoxy)3 -vinylbenzene $(0.20 \mathrm{mmol}, 38 \mathrm{mg})$, and [1.1.1]propellane $(0.80 \mathrm{mmol})$ in toluene (3 $\mathrm{mL})$. Purification by flash column chromatography with pentane as eluent gave $7 \mathrm{~g}$ (37 mg, 72\% yield) as a colourless oil. IR (film) $v_{\max } / \mathrm{cm}^{-1}: 3072,2991,2934$, 2907, 1613, 1256, 1216, 1161, 878, 700. HRMS (APCI): Calcd. for $\mathrm{C}_{14} \mathrm{H}_{14} \mathrm{~F}_{3} \mathrm{O}$ $\left([\mathrm{M}+\mathrm{H}]^{+}\right) \mathrm{m} / \mathrm{z}$ 255.0991, found m/z 255.0991. ${ }^{1} \mathrm{H}$ NMR $(400 \mathrm{MHz}$, Chloroform- $d$ ) $\delta 7.29(\mathrm{t}, J=8.0$ $\mathrm{Hz}, 1 \mathrm{H}), 7.06-7.00(\mathrm{~m}, 1 \mathrm{H}), 6.93(\mathrm{~d}, J=7.8 \mathrm{~Hz}, 1 \mathrm{H}), 6.80(\mathrm{~s}, 1 \mathrm{H}), 4.87(\mathrm{p}, J=2.6 \mathrm{~Hz}, 1 \mathrm{H}), 4.84$ (p, $J=2.5 \mathrm{~Hz}, 1 \mathrm{H}), 2.96(\mathrm{dq}, J=15.9,2.7 \mathrm{~Hz}, 1 \mathrm{H}), 2.88(\mathrm{dq}, J=15.9,2.6 \mathrm{~Hz}, 1 \mathrm{H}), 2.76(\mathrm{dq}, J=16.1$, $2.6 \mathrm{~Hz}, 1 \mathrm{H}), 2.60(\mathrm{dq}, J=16.1,2.8 \mathrm{~Hz}, 1 \mathrm{H}), 2.02(\mathrm{dd}, J=8.8,5.9 \mathrm{~Hz}, 1 \mathrm{H}), 1.25(\mathrm{dd}, J=8.9,5.6 \mathrm{~Hz}$, $1 \mathrm{H}), 0.99(\mathrm{t}, J=5.7 \mathrm{~Hz}, 1 \mathrm{H}) .{ }^{13} \mathrm{C}$ NMR $(100 \mathrm{MHz}$, Chloroform- $d) \delta 149.3(\mathrm{q}, J=1.9 \mathrm{~Hz}), 143.9$, 143.6, 129.3, 125.1, 120.5 (d, $J=254.7 \mathrm{~Hz}), 118.9,117.6,106.6,40.5,36.0,27.5,25.1,20.1 .{ }^{19} \mathrm{~F}$ NMR $\left(377 \mathrm{MHz}, \mathrm{CDCl}_{3}\right) \delta-57.7$. 


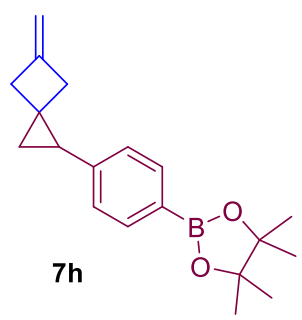

Prepared following General Procedure A, using Ni(cod) $)_{2}(10 \mathrm{~mol} \%, 5.6 \mathrm{mg})$, SIMes $\cdot \mathrm{HCl}(12 \mathrm{~mol} \%, 8.3 \mathrm{mg})$, LiOMe (20 mol\%, $1.5 \mathrm{mg}), 4,4,5,5$-tetramethyl2-(4-vinylphenyl)-1,3,2-dioxaborolane $\quad(0.20 \mathrm{mmol}, \quad 46 \mathrm{mg}), \quad$ and [1.1.1]propellane $(0.80 \mathrm{mmol})$ in toluene $(3 \mathrm{~mL})$. Purification by flash column chromatography with pentane/diethyl ether (50/1) as eluent gave $7 \mathbf{h}$ (28 $\mathrm{mg}$, $47 \%$ yield) as a colourless oil. IR (film) $v_{\max } / \mathrm{cm}^{-1}: 2978,2931,2904,1677,1610$, 1398, 1358, 1143, 1091, 859. HRMS (APCI): Calcd. for $\mathrm{C}_{19} \mathrm{H}_{26} \mathrm{O}_{3} \mathrm{~B}\left([\mathrm{M}+\mathrm{H}]^{+}\right)$ $\mathrm{m} / \mathrm{z}$ 297.2020, found $\mathrm{m} / \mathrm{z}$ 297.2017. ${ }^{1} \mathrm{H}$ NMR (400 MHz, Chloroform- $d$ ) $\delta 7.71(\mathrm{~d}, J=8.0 \mathrm{~Hz}, 2 \mathrm{H})$, $6.96(\mathrm{~d}, J=8.0 \mathrm{~Hz}, 2 \mathrm{H}), 4.81(\mathrm{p}, J=2.5 \mathrm{~Hz}, 1 \mathrm{H}), 4.78(\mathrm{p}, J=2.5 \mathrm{~Hz}, 1 \mathrm{H}), 2.93(\mathrm{dq}, J=15.9,2.9 \mathrm{~Hz}$, $1 \mathrm{H}), 2.85(\mathrm{dq}, J=15.9,2.8 \mathrm{~Hz}, 1 \mathrm{H}), 2.72(\mathrm{dq}, J=16.2,2.6 \mathrm{~Hz}, 1 \mathrm{H}), 2.57$ (dq, $J=16.0,2.5 \mathrm{~Hz}, 1 \mathrm{H})$, $1.99(\mathrm{dd}, J=8.8,6.0 \mathrm{~Hz}, 1 \mathrm{H}), 1.34(\mathrm{~s}, 12 \mathrm{H}), 1.19(\mathrm{dd}, J=8.9,5.4 \mathrm{~Hz}, 1 \mathrm{H}), 1.02(\mathrm{t}, J=5.7 \mathrm{~Hz}, 1 \mathrm{H})$. ${ }^{13} \mathrm{C}$ NMR (100 MHz, Chloroform- $d$ ) $\delta$ 144.5, 144.4, 134.6, 126.0, 106.3, 83.6, 40.7, 36.1, 28.1, 25.2, $24.9,19.9$, the signal for the carbon adjacent to boron could not be observed due to quadrupolar relaxation.

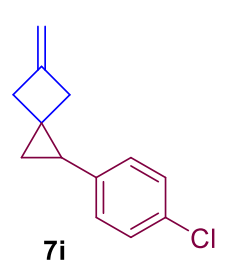

Prepared following General Procedure A, using $\mathrm{Ni}(\mathrm{cod})_{2}(10 \mathrm{~mol} \%$, $5.6 \mathrm{mg})$, SIMes $\cdot \mathrm{HCl}(12 \mathrm{~mol} \%, 8.3 \mathrm{mg})$, LiOMe (20 mol\%, $1.5 \mathrm{mg})$, 1-chloro-4-vinylbenzene $(0.20 \mathrm{mmol}, 28 \mathrm{mg})$, and [1.1.1]propellane $(0.80 \mathrm{mmol})$ in toluene $(3 \mathrm{~mL})$. Purification by flash column chromatography with pentane as eluent gave $7 \mathbf{i}$ (30.7 $\mathrm{mg}, 75 \%$ yield) as a colourless oil. IR (film) $v_{\max } / \mathrm{cm}^{-1}: 3063,2987,2938,2904,1677$, 1493, 1092, 1013, 878, 821. HRMS (APCI): Calcd. for $\mathrm{C}_{13} \mathrm{H}_{14} \mathrm{Cl}\left([\mathrm{M}+\mathrm{H}]^{+}\right) \mathrm{m} / \mathrm{z}$ 205.0779, found $\mathrm{m} / \mathrm{z}$ 205.0779. ${ }^{1} \mathrm{H}$ NMR (400 MHz, Chloroform- $d$ ) $\delta 7.26-7.21(\mathrm{~m}, 2 \mathrm{H}), 6.93-$ $7.87(\mathrm{~m}, 2 \mathrm{H}), 4.84(\mathrm{p}, J=2.3 \mathrm{~Hz}, 1 \mathrm{H}), 4.81(\mathrm{p}, J=2.5 \mathrm{~Hz}, 1 \mathrm{H}), 2.92(\mathrm{dq}, J=15.9,2.7 \mathrm{~Hz}, 1 \mathrm{H}), 2.85$ $(\mathrm{dq}, J=15.9,2.7 \mathrm{~Hz}, 1 \mathrm{H}), 2.70(\mathrm{dq}, J=16.1,2.6 \mathrm{~Hz}, 1 \mathrm{H}), 2.55(\mathrm{dq}, J=16.1,2.8 \mathrm{~Hz}, 1 \mathrm{H}), 1.95(\mathrm{dd}, J$ $=8.9,5.9 \mathrm{~Hz}, 1 \mathrm{H}), 1.19(\mathrm{dd}, J=8.9,5.5 \mathrm{~Hz}, 1 \mathrm{H}), 0.93(\mathrm{t}, J=5.7 \mathrm{~Hz}, 1 \mathrm{H}) .{ }^{13} \mathrm{C}$ NMR $(100 \mathrm{MHz}$, Chloroform- $d$ ) $\delta 144.2,139.5,131.0,128.2,128.0,106.6,40.6,36.0,27.2,24.8,19.8$.

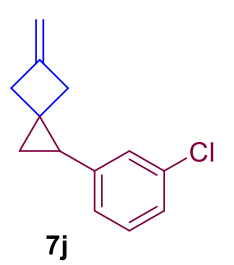

Prepared following General Procedure A, using $\mathrm{Ni}(\mathrm{cod})_{2}(10 \mathrm{~mol} \%$, $5.6 \mathrm{mg})$, SIMes $\cdot \mathrm{HCl}(12 \mathrm{~mol} \%, 8.3 \mathrm{mg})$, LiOMe (20 mol\%, $1.5 \mathrm{mg})$, 1-chloro-3-vinylbenzene $(0.20 \mathrm{mmol}, 28 \mathrm{mg})$, and [1.1.1]propellane $(0.80 \mathrm{mmol})$ in toluene $(3 \mathrm{~mL})$. Purification by flash column chromatography with pentane as eluent gave $\mathbf{7 j}$ (30 $\mathrm{mg}$, $74 \%$ yield) as a colourless oil. IR (film) $v_{\max } / \mathrm{cm}^{-1}: 3071,2989,2934,2904,1678$, 1598, 1569, 1479, 1082, 877, 778. HRMS (APCI): Calcd. for $\mathrm{C}_{13} \mathrm{H}_{14} \mathrm{Cl}\left([\mathrm{M}+\mathrm{H}]^{+}\right) \mathrm{m} / \mathrm{z}$ 205.0779, found $\mathrm{m} / \mathrm{z}$ 205.0776. ${ }^{1} \mathrm{H}$ NMR $(400 \mathrm{MHz}$, Chloroform- $d$ ) $\delta 7.19(\mathrm{t}, J=7.8 \mathrm{~Hz}, 1 \mathrm{H}), 7.13$ $(\mathrm{ddd}, J=8.0,2.0,1.2 \mathrm{~Hz}, 1 \mathrm{H}), 6.95(\mathrm{t}, J=1.8 \mathrm{~Hz}, 1 \mathrm{H}), 6.84(\mathrm{dt}, J=7.6,1.2 \mathrm{~Hz}, 1 \mathrm{H}), 4.84(\mathrm{p}, J=2.1$ $\mathrm{Hz}, 1 \mathrm{H}), 4.82(\mathrm{p}, J=2.1 \mathrm{~Hz}, 1 \mathrm{H}), 2.93(\mathrm{dq}, J=15.9,2.7 \mathrm{~Hz}, 1 \mathrm{H}), 2.89-2.82(\mathrm{~m}, 1 \mathrm{H}), 2.73(\mathrm{dq}, J=$ $16.1,2.6 \mathrm{~Hz}, 1 \mathrm{H}), 2.58(\mathrm{dq}, J=16.1,2.8 \mathrm{~Hz}, 1 \mathrm{H}), 1.96(\mathrm{dd}, J=8.8,5.9 \mathrm{~Hz}, 1 \mathrm{H}), 1.20(\mathrm{dd}, J=8.9,5.6$ $\mathrm{Hz}, 1 \mathrm{H}), 0.97(\mathrm{t}, J=5.7 \mathrm{~Hz}, 1 \mathrm{H}) .{ }^{13} \mathrm{C}$ NMR $(100 \mathrm{MHz}$, Chloroform- $d$ ) $\delta 144.1,143.3,134.1,129.4$, $126.8,125.5,124.9,106.7,40.6,36.1,27.5,25.1,19.9$.

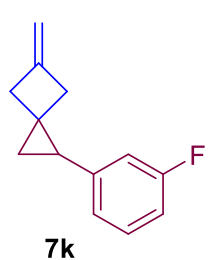

Prepared following General Procedure A, using $\mathrm{Ni}(\operatorname{cod})_{2}(10 \mathrm{~mol} \%, 5.6 \mathrm{mg})$, SIMes $\cdot \mathrm{HCl}(12 \mathrm{~mol} \%, 8.3 \mathrm{mg})$, LiOMe (20 mol\%, $1.5 \mathrm{mg})$, 1-fluoro-3-vinylbenzene $(0.20 \mathrm{mmol}, 25 \mathrm{mg})$, and [1.1.1]propellane $(0.80 \mathrm{mmol})$ in toluene $(3 \mathrm{~mL})$. Purification by flash column chromatography with pentane as eluent gave $7 \mathbf{k}(29 \mathrm{mg}$, $77 \%$ yield) as a colourless oil. IR (film) $v_{\max } / \mathrm{cm}^{-1}: 3072,2989,2937,2905,1678,1616$, 1587, 1488, 1143, 875, 781. HRMS (APCI): Calcd. for $\mathrm{C}_{13} \mathrm{H}_{14} \mathrm{~F}\left([\mathrm{M}+\mathrm{H}]^{+}\right) \mathrm{m} / \mathrm{z}$ 189.1074, found $\mathrm{m} / \mathrm{z} 189.1070 .{ }^{1} \mathrm{H}$ NMR (400 MHz, Chloroform- $d$ ) $\delta 7.25-7.18(\mathrm{~m}, 1 \mathrm{H}), 6.84(\mathrm{td}, J$ 
$=8.5,2.5 \mathrm{~Hz}, 1 \mathrm{H}), 6.77(\mathrm{~d}, J=7.8 \mathrm{~Hz}, 1 \mathrm{H}), 6.63(\mathrm{~d}, J=10.5 \mathrm{~Hz}, 1 \mathrm{H}), 4.87-4.79(\mathrm{~m}, 2 \mathrm{H}), 2.93(\mathrm{dq}$, $\mathrm{J}=15.9,2.5 \mathrm{~Hz}, 1 \mathrm{H}), 2.85(\mathrm{dq}, \mathrm{J}=16.0,2.5 \mathrm{~Hz}, 1 \mathrm{H}), 2.73(\mathrm{dq}, J=16.1,2.5 \mathrm{~Hz}, 1 \mathrm{H}), 2.61(\mathrm{dq}, J=$ $15.8,2.6 \mathrm{~Hz}, 1 \mathrm{H}), 1.98(\mathrm{dd}, J=8.8,6.0 \mathrm{~Hz}, 1 \mathrm{H}), 1.21(\mathrm{dd}, J=8.9,5.5 \mathrm{~Hz}, 1 \mathrm{H}), 0.96(\mathrm{t}, J=5.7 \mathrm{~Hz}$, 1H). ${ }^{13} \mathrm{C}$ NMR $(100 \mathrm{MHz}$, Chloroform- $d) \delta 163.0(\mathrm{~d}, J=243.3 \mathrm{~Hz}), 144.2,143.9(\mathrm{~d}, J=7.5 \mathrm{~Hz})$, $129.5(\mathrm{~d}, J=8.6 \mathrm{~Hz}), 122.5(\mathrm{~d}, J=2.7 \mathrm{~Hz}), 113.3(\mathrm{~d}, J=21.5 \mathrm{~Hz}), 112.2(\mathrm{~d}, J=21.1 \mathrm{~Hz}), 106.6,40.6$, 36.1, 27.6 (d, $J=2.2 \mathrm{~Hz}), 25.1,20.0 .{ }^{19} \mathrm{~F}$ NMR $(376 \mathrm{MHz}$, Chloroform- $d$ ) $\delta-113.9$.

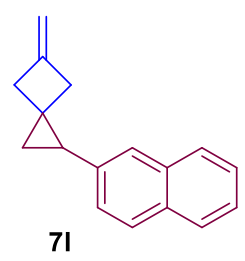

Prepared following General Procedure A, using Ni(cod) $)_{2}(10 \mathrm{~mol} \%, 5.6 \mathrm{mg})$, SIMes $\cdot \mathrm{HCl}$ (12 mol\%, $8.3 \mathrm{mg}$ ), LiOMe (20 mol\%, $1.5 \mathrm{mg})$, 2-vinylnaphthalene $(0.20 \mathrm{mmol}, 31 \mathrm{mg})$, and [1.1.1]propellane $(0.80 \mathrm{mmol})$ in toluene $(3 \mathrm{~mL})$. Purification by flash column chromatography with pentane as eluent gave 71 ( $37 \mathrm{mg}$, $84 \%$ yield) as a colourless oil. IR (film) $v_{\max } / \mathrm{cm}^{-1}: 3056,2985,2931,2903,1676$, 1633, 1599, 1508, 877, 814, 747. HRMS (APCI): Calcd. for $\mathrm{C}_{17} \mathrm{H}_{17}\left([\mathrm{M}+\mathrm{H}]^{+}\right) \mathrm{m} / \mathrm{z}$ 221.1325, found $\mathrm{m} / \mathrm{z}$ 221.1321. ${ }^{1} \mathrm{H}$ NMR (400 MHz, Chloroform- $d$ ) $\delta 7.81-7.72(\mathrm{~m}, 3 \mathrm{H}), 7.48-$ $7.36(\mathrm{~m}, 3 \mathrm{H}), 7.12(\mathrm{~d}, J=1.6 \mathrm{~Hz}, 1 \mathrm{H}), 4.84(\mathrm{p}, J=2.4 \mathrm{~Hz}, 1 \mathrm{H}), 4.79$ (p, $J=2.4 \mathrm{~Hz}, 1 \mathrm{H}), 2.98$ (dq, $J=$ 15.8, $2.6 \mathrm{~Hz}, 1 \mathrm{H}), 2.90(\mathrm{dq}, J=15.9,2.6 \mathrm{~Hz}, 1 \mathrm{H}), 2.75(\mathrm{dq}, J=16.2,2.7 \mathrm{~Hz}, 1 \mathrm{H}), 2.59$ (dq, $J=16.1$, $2.8 \mathrm{~Hz}, 1 \mathrm{H}), 2.15(\mathrm{dd}, J=8.8,6.0 \mathrm{~Hz}, 1 \mathrm{H}), 1.24(\mathrm{dd}, J=8.8,5.5 \mathrm{~Hz}, 1 \mathrm{H}), 1.13(\mathrm{t}, J=5.7 \mathrm{~Hz}, 1 \mathrm{H}) .{ }^{13} \mathrm{C}$ NMR (100 MHz, Chloroform- $d$ ) $\delta$ 144.6, 138.6, 133.6, 131.9, 127.7, 127.6, 127.4, 126.0, 125.9, 125.0, 124.7, 106.5, 40.8, 36.3, 28.0, 25.0, 19.6.

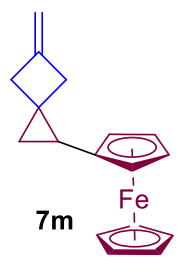

Prepared following General Procedure A, using Ni(cod $)_{2}(10 \mathrm{~mol} \%, 5.6 \mathrm{mg})$, SIMes $\cdot \mathrm{HCl}$ (12 mol\%, $8.3 \mathrm{mg}$ ), LiOMe (20 mol\%, $1.5 \mathrm{mg})$, 1-vinyl-ferrocene (0.20 mmol, $43 \mathrm{mg}$ ), and [1.1.1]propellane $(0.80 \mathrm{mmol})$ in toluene $(3 \mathrm{~mL})$. Purification by flash column chromatography with pentane as eluent gave $7 \mathbf{m}(25 \mathrm{mg}, 44 \%$ yield $)$ as a yellow oil. IR (film) $v_{\max } / \mathrm{cm}^{-1}: 3092,2986,2902,1676,1105,1000,876,816$. HRMS (Nanospray): Calcd. for $\mathrm{C}_{17} \mathrm{H}_{18} \mathrm{Fe}\left([\mathrm{M}]^{+}\right) \mathrm{m} / \mathrm{z}$ 278.0758, found $\mathrm{m} / \mathrm{z}$ 278.0762. ${ }^{1} \mathrm{H}$ NMR (400 MHz, Chloroform- $d$ ) $\delta 4.84-4.78(\mathrm{~m}, 2 \mathrm{H}), 4.13(\mathrm{~s}, 5 \mathrm{H}), 4.08-3.99(\mathrm{~m}, 3 \mathrm{H}), 3.77(\mathrm{dd}, J=2.2,1.1 \mathrm{~Hz}, 1 \mathrm{H})$, $2.86-2.69(\mathrm{~m}, 3 \mathrm{H}), 2.63(\mathrm{dq}, J=16.8,3.3,2.9 \mathrm{~Hz}, 1 \mathrm{H}), 1.64(\mathrm{dd}, J=9.0,5.8 \mathrm{~Hz}, 1 \mathrm{H}), 1.04(\mathrm{dd}, J=$ 9.1, 5.0 Hz, 1H), $0.61(\mathrm{t}, J=5.4 \mathrm{~Hz}, 1 \mathrm{H}) .{ }^{13} \mathrm{C}$ NMR $(100 \mathrm{MHz}$, Chloroform- $d$ ) $\delta$ 144.9, 106.2, 89.0, $68.5,67.9,67.1,66.6,65.7,40.4,36.5,24.6,22.4,19.9$.

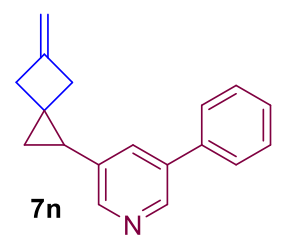

Prepared following General Procedure A, using Ni(cod) $)_{2}(10 \mathrm{~mol} \%, 5.6 \mathrm{mg})$, SIMes $\cdot \mathrm{HCl}$ (12 mol\%, $8.3 \mathrm{mg})$, LiOMe (20 mol\%, $1.5 \mathrm{mg})$, 3-phenyl-5vinylpyridine $(0.20 \mathrm{mmol}, 37 \mathrm{mg})$, and [1.1.1]propellane $(0.80 \mathrm{mmol})$ in toluene ( $3 \mathrm{~mL}$ ). Purification by flash column chromatography with pentane/diethyl ether (20/1) as eluent gave 7n (24 mg, 49\% yield) as a colourless oil. IR (film) $v_{\max } / \mathrm{cm}^{-}$ ${ }^{1}$ : 3060, 3034, 2988, 2903, 1677, 1593, 1418, 880, 762. HRMS (APCI): Calcd. for $\mathrm{C}_{18} \mathrm{H}_{18} \mathrm{~N}\left([\mathrm{M}+\mathrm{H}]^{+}\right)$ $\mathrm{m} / \mathrm{z}$ 248.1434, found m/z 248.1430. ${ }^{1} \mathrm{H}$ NMR (400 MHz, Chloroform- $d$ ) $\delta 8.63(\mathrm{~d}, J=2.1 \mathrm{~Hz}, 1 \mathrm{H})$, $8.32(\mathrm{~d}, J=2.1 \mathrm{~Hz}, 1 \mathrm{H}), 7.58-7.53(\mathrm{~m}, 2 \mathrm{H}), 7.51-7.44(\mathrm{~m}, 2 \mathrm{H}), 7.44-7.37(\mathrm{~m}, 1 \mathrm{H}), 7.34(\mathrm{t}, J=$ $2.1 \mathrm{~Hz}, 1 \mathrm{H}), 4.86(\mathrm{p}, J=2.3 \mathrm{~Hz}, 1 \mathrm{H}), 4.84(\mathrm{p}, J=2.6 \mathrm{~Hz}, 1 \mathrm{H}), 2.98(\mathrm{dq}, J=15.9,2.7 \mathrm{~Hz}, 1 \mathrm{H}), 2.90$ $(\mathrm{dq}, J=15.9,2.6 \mathrm{~Hz}, 1 \mathrm{H}), 2.78(\mathrm{dq}, J=16.0,2.6 \mathrm{~Hz}, 1 \mathrm{H}), 2.64(\mathrm{dq}, J=16.0,2.8 \mathrm{~Hz}, 1 \mathrm{H}), 2.05(\mathrm{dd}, J$ $=9.0,6.0 \mathrm{~Hz}, 1 \mathrm{H}), 1.30(\mathrm{dd}, J=8.9,5.6 \mathrm{~Hz}, 1 \mathrm{H}), 1.06(\mathrm{t}, J=5.7 \mathrm{~Hz}, 1 \mathrm{H}) .{ }^{1} \mathrm{H}$ NMR $(100 \mathrm{MHz}$, Chloroform- $d$ ) $\delta 147.7,145.5,143.6,138.2,136.6,136.1,131.9,129.1,128.1,127.3,107.0,40.6$, $36.1,25.2,25.2,19.8$. 


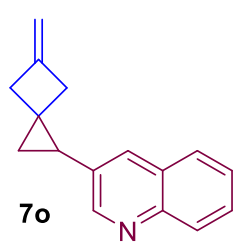

Prepared following General Procedure A, using $\mathrm{Ni}(\mathrm{cod})_{2}(10 \mathrm{~mol} \%$, $5.6 \mathrm{mg})$, SIMes $\cdot \mathrm{HCl}$ (12 mol\%, $8.3 \mathrm{mg})$, LiOMe (20 mol\%, $1.5 \mathrm{mg}), 3$-vinylquinoline $(0.20$ $\mathrm{mmol}, 31 \mathrm{mg})$, and [1.1.1]propellane $(0.80 \mathrm{mmol})$ in toluene $(3 \mathrm{~mL})$. Purification by flash column chromatography with pentane/diethyl ether (20/1) as eluent gave 7o (22 mg, 50\% yield) as a colourless oil. IR (film) $v_{\max } / \mathrm{cm}^{-1}: 3065,2987,2902,1677$, 1493, 879, 786, 751. HRMS (APCI): Calcd. for $\mathrm{C}_{16} \mathrm{H}_{16} \mathrm{~N}\left([\mathrm{M}+\mathrm{H}]^{+}\right) \mathrm{m} / \mathrm{z} 222.1277$, found $\mathrm{m} / \mathrm{z}$ 222.1275. ${ }^{1} \mathrm{H}$ NMR (400 MHz, Chloroform- $d$ ) $\delta 8.69(\mathrm{~d}, J=2.3 \mathrm{~Hz}, 1 \mathrm{H}), 8.06(\mathrm{~d}, J=8.4$ $\mathrm{Hz}, 1 \mathrm{H}), 7.73(\mathrm{~d}, J=8.1 \mathrm{~Hz}, 1 \mathrm{H}), 7.63(\mathrm{ddd}, J=8.4,6.9,1.4 \mathrm{~Hz}, 1 \mathrm{H}), 7.58$ (d, $J=2.1 \mathrm{~Hz}, 1 \mathrm{H}), 7.54-$ $7.48(\mathrm{~m}, 1 \mathrm{H}), 4.86(\mathrm{p}, J=2.5 \mathrm{~Hz}, 1 \mathrm{H}), 4.81$ (p, $J=2.5 \mathrm{~Hz}, 1 \mathrm{H}), 3.01$ (dq, $J=15.9,2.8 \mathrm{~Hz}, 1 \mathrm{H}), 2.92$ (dq, $J=16.0,2.6 \mathrm{~Hz}, 1 \mathrm{H}), 2.77$ (dq, $J=16.1,2.6 \mathrm{~Hz}, 1 \mathrm{H}), 2.57$ (dq, $J=15.9,2.7 \mathrm{~Hz}, 1 \mathrm{H}), 2.17$ (dd, $J$ $=8.9,5.9 \mathrm{~Hz}, 1 \mathrm{H}), 1.34(\mathrm{dd}, J=8.9,5.6 \mathrm{~Hz}, 1 \mathrm{H}), 1.15(\mathrm{t}, J=5.8 \mathrm{~Hz}, 1 \mathrm{H}) .{ }^{13} \mathrm{C} \mathrm{NMR}(100 \mathrm{MHz}$, Chloroform- $d$ ) $\delta 151.2,146.7,143.7,134.0,131.6,129.3,128.4,128.0,127.3,126.7,106.9,40.6$, $36.03,25.6,25.4,19.7$.

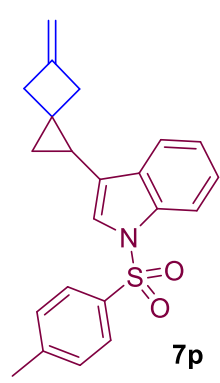

Prepared following General Procedure A, using $\mathrm{Ni}(\mathrm{cod})_{2}(10 \mathrm{~mol} \%$, $5.6 \mathrm{mg})$, SIMes $\cdot \mathrm{HCl}(12 \mathrm{~mol} \%, 8.3 \mathrm{mg})$, LiOMe (20 mol\%, $1.5 \mathrm{mg})$, 1-tosyl-3-vinyl-1Hindole $(0.20 \mathrm{mmol}, 60 \mathrm{mg})$, and [1.1.1]propellane $(0.80 \mathrm{mmol})$ in toluene $(3 \mathrm{~mL})$. Purification by flash column chromatography with toluene/hexane (1/2) as eluent gave 7p (61 mg, 84\% yield) as a colourless oil. IR (film) $v_{\max } / \mathrm{cm}^{-1}: 3066,2986,2903$, 1446, 1368, 1173, 1123, 747, 669. HRMS (APCI): Calcd. for $\mathrm{C}_{22} \mathrm{H}_{22} \mathrm{NO}_{2} \mathrm{~S}\left([\mathrm{M}+\mathrm{H}]^{+}\right)$ $\mathrm{m} / \mathrm{z}$ 364.1366, found m/z 364.1356. ${ }^{1} \mathrm{H}$ NMR (400 MHz, Chloroform- $d$ ) $\delta 7.98-7.94$ $(\mathrm{m}, 1 \mathrm{H}), 7.71-7.66(\mathrm{~m}, 2 \mathrm{H}), 7.59-7.54(\mathrm{~m}, 1 \mathrm{H}), 7.31(\mathrm{td}, J=8.3,1.2 \mathrm{~Hz}, 1 \mathrm{H}), 7.24$ $(\mathrm{td}, J=7.6,1.1 \mathrm{~Hz}, 1 \mathrm{H}), 7.17(\mathrm{~d}, J=8.0 \mathrm{~Hz}, 2 \mathrm{H}), 7.04(\mathrm{~d}, J=0.9 \mathrm{~Hz}, 1 \mathrm{H}), 4.83(\mathrm{p}, J$ $=2.5 \mathrm{~Hz}, 1 \mathrm{H}), 4.75(\mathrm{p}, J=2.5 \mathrm{~Hz}, 1 \mathrm{H}), 2.95(\mathrm{dq}, J=15.7,2.7 \mathrm{~Hz}, 1 \mathrm{H}), 2.89(\mathrm{dq}, J=15.8,2.7 \mathrm{~Hz}$, $1 \mathrm{H}), 2.53(\mathrm{dq}, J=16.2,2.7 \mathrm{~Hz}, 1 \mathrm{H}), 2.36-2.27(\mathrm{~m}, 4 \mathrm{H}), 1.92(\mathrm{dd}, J=8.6,6.1 \mathrm{~Hz}, 1 \mathrm{H}), 1.19(\mathrm{dd}, J=$ 8.8, $5.2 \mathrm{~Hz}, 1 \mathrm{H}), 0.94(\mathrm{t}, J=5.5 \mathrm{~Hz}, 1 \mathrm{H}) .{ }^{13} \mathrm{C}$ NMR (100 MHz, Chloroform- $d$ ) $\delta 144.8,144.2,135.4$, 135.2, 132.1, 129.8, 126.8, 124.9, 123.6, 123.3, 121.7, 119.7, 113.9, 106.7, 40.1, 36.2, 23.6, 21.6, 18.0, 17.9.

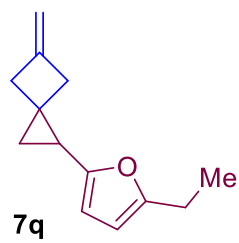

Prepared following General Procedure A, using $\mathrm{Ni}(\operatorname{cod})_{2}(10 \mathrm{~mol} \%, 5.6 \mathrm{mg})$, SIMes $\cdot \mathrm{HCl}(12 \mathrm{~mol} \%, 8.3 \mathrm{mg}$ ), LiOMe (20 mol\%, $1.5 \mathrm{mg}$ ), 2-ethyl-5-vinylfuran $(0.20 \mathrm{mmol}, 25 \mathrm{mg})$, and [1.1.1]propellane $(0.80 \mathrm{mmol})$ in toluene $(3 \mathrm{~mL})$. Purification by flash column chromatography with pentane as eluent gave $\mathbf{7 q}$ ( 28 $\mathrm{mg}, 73 \%$ yield) as a colourless oil. IR (film) $v_{\max } / \mathrm{cm}^{-1}: 3072,2973,2941,2906$, 1677, 1177, 1011, 877, 774. HRMS (APCI): Calcd. for $\mathrm{C}_{13} \mathrm{H}_{17} \mathrm{O}\left([\mathrm{M}+\mathrm{H}]^{+}\right) \mathrm{m} / \mathrm{z}$ 189.1274, found $\mathrm{m} / \mathrm{z}$ 189.1272. ${ }^{1} \mathrm{H}$ NMR (400 MHz, Chloroform- $d$ ) $\delta 5.88-5.82(\mathrm{~m}, 1 \mathrm{H}), 5.77(\mathrm{~d}, J$ $=3.0 \mathrm{~Hz}, 1 \mathrm{H}), 4.85-4.80(\mathrm{~m}, 2 \mathrm{H}), 2.92(\mathrm{dp}, J=15.8,2.4 \mathrm{~Hz}, 1 \mathrm{H}), 2.79(\mathrm{dp}, J=15.7,2.3 \mathrm{~Hz}, 1 \mathrm{H})$, $2.71-2.67(\mathrm{~m}, 2 \mathrm{H}), 2.58(\mathrm{qd}, J=7.5,1.0 \mathrm{~Hz}, 2 \mathrm{H}), 1.93(\mathrm{dd}, J=9.1,5.8 \mathrm{~Hz}, 1 \mathrm{H}), 1.20(\mathrm{t}, J=7.5 \mathrm{~Hz}$, $3 \mathrm{H}), 1.08(\mathrm{dd}, J=9.1,5.0 \mathrm{~Hz}, 1 \mathrm{H}), 0.95(\mathrm{t}, J=5.4 \mathrm{~Hz}, 1 \mathrm{H}) .{ }^{13} \mathrm{C}$ NMR $(100 \mathrm{MHz}$, Chloroform- $d$ ) $\delta$ 156.0, 153.6, 144.7, 106.4, 104.6, 104.3, 39.9, 36.9, 23.5, 21.5, 20.7, 18.0, 12.3.

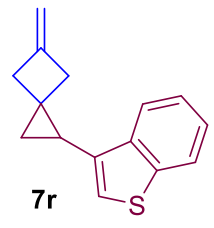

Prepared following General Procedure A, using Ni(cod) $)_{2}(10 \mathrm{~mol} \%$, $5.6 \mathrm{mg})$, SIMes $\cdot \mathrm{HCl}(12 \mathrm{~mol} \%, 8.3 \mathrm{mg})$, LiOMe (20 mol\%, $1.5 \mathrm{mg})$, 3-vinylbenzo[ $b]$ thiophene $(0.20 \mathrm{mmol}, 32 \mathrm{mg})$, and [1.1.1]propellane $(0.80 \mathrm{mmol})$ in toluene $(3 \mathrm{~mL})$. Purification by flash column chromatography with pentane as eluent gave $7 \mathbf{r}$ (44 mg, $91 \%$ yield) as a colourless oil. IR (film) $v_{\max } / \mathrm{cm}^{-1}: 3067,2987,2931,2902,1677,877$, 763, 732. HRMS (APCI): Calcd. for $\mathrm{C}_{15} \mathrm{H}_{15} \mathrm{~S}\left([\mathrm{M}+\mathrm{H}]^{+}\right) \mathrm{m} / \mathrm{z} 227.0889$, found $\mathrm{m} / \mathrm{z} 227.0884$. ${ }^{1} \mathrm{H}$ NMR 
(400 MHz, Chloroform- $d$ ) $\delta 7.90(\mathrm{~d}, J=8.0 \mathrm{~Hz}, 1 \mathrm{H}), 7.85(\mathrm{~d}, J=8.7 \mathrm{~Hz}, 1 \mathrm{H}), 7.42(\mathrm{t}, J=7.2 \mathrm{~Hz}, 1 \mathrm{H})$, $7.37(\mathrm{t}, J=7.8 \mathrm{~Hz}, 1 \mathrm{H}), 6.84(\mathrm{~s}, 1 \mathrm{H}), 4.86(\mathrm{p}, J=2.5 \mathrm{~Hz}, 1 \mathrm{H}), 4.77(\mathrm{p}, J=2.5 \mathrm{~Hz}, 1 \mathrm{H}), 3.06(\mathrm{dq}, J=$ $15.9,2.6 \mathrm{~Hz}, 1 \mathrm{H}), 2.96(\mathrm{dq}, J=15.9,2.6 \mathrm{~Hz}, 1 \mathrm{H}), 2.57(\mathrm{dq}, J=16.2,2.6 \mathrm{~Hz}, 1 \mathrm{H}), 2.40(\mathrm{dq}, J=16.1$, $2.7 \mathrm{~Hz}, 1 \mathrm{H}), 2.16(\mathrm{dd}, J=8.5,6.2 \mathrm{~Hz}, 1 \mathrm{H}), 1.24(\mathrm{dd}, J=8.7,5.2 \mathrm{~Hz}, 1 \mathrm{H}), 1.08(\mathrm{t}, J=5.6 \mathrm{~Hz}, 1 \mathrm{H}) .{ }^{13} \mathrm{C}$ NMR (100 MHz, Chloroform- $d$ ) $\delta$ 144.4, 140.4, 140.1, 136.3, 124.3, 124.0, 122.8, 122.0, 120.0, 106.4, $40.4,36.4,23.4,21.3,17.6$.

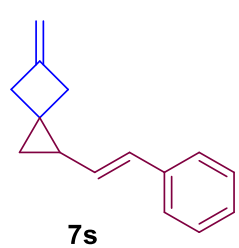

Prepared following General Procedure B, using Ni(cod) $)_{2}(10 \mathrm{~mol} \%, 5.6 \mathrm{mg})$, SIMes:HCl (12 mol\%, $8.3 \mathrm{mg})$, LiOMe (20 mol\%, $1.5 \mathrm{mg})$, (E)-buta-1,3-dien-1ylbenzene $(0.20 \mathrm{mmol}, 26 \mathrm{mg})$, and [1.1.1]propellane $(0.80 \mathrm{mmol})$ in toluene $(3$ $\mathrm{mL}$ ). Purification by flash column chromatography with pentane as eluent gave $\mathbf{7 s}$ (32 mg, 82\% yield) as a colourless oil. IR (film) $v_{\max } / \mathrm{cm}^{-1}: 3059,3024,2986,2903$, 1677, 1495, 954, 876, 748, 692. HRMS (APCI): Calcd. for $\mathrm{C}_{15} \mathrm{H}_{17}\left([\mathrm{M}+\mathrm{H}]^{+}\right) \mathrm{m} / \mathrm{z}$ 197.1325, found $\mathrm{m} / \mathrm{z}$ 197.1325. ${ }^{1} \mathrm{H}$ NMR (400 MHz, Chloroform- $d$ ) $\delta 7.26-7.15(\mathrm{~m}, 4 \mathrm{H}), 7.13-$ $7.06(\mathrm{~m}, 1 \mathrm{H}), 6.37(\mathrm{~d}, J=15.8 \mathrm{~Hz}, 1 \mathrm{H}), 5.64(\mathrm{dd}, J=15.8,9.1 \mathrm{~Hz}, 1 \mathrm{H}), 4.79-4.73(\mathrm{~m}, 2 \mathrm{H}), 2.89-$ $2.62(\mathrm{~m}, 4 \mathrm{H}), 1.55(\mathrm{td}, J=8.8,5.3 \mathrm{~Hz}, 1 \mathrm{H}), 0.97(\mathrm{dd}, J=8.6,5.1 \mathrm{~Hz}, 1 \mathrm{H}), 0.63(\mathrm{t}, J=5.2 \mathrm{~Hz}, 1 \mathrm{H})$. ${ }^{13} \mathrm{C}$ NMR (100 MHz, Chloroform- $d$ ) $\delta 145.0,137.8,131.8,128.6,128.5,126.6,125.6,106.4,40.5$, $36.4,26.0,23.6,20.2$.

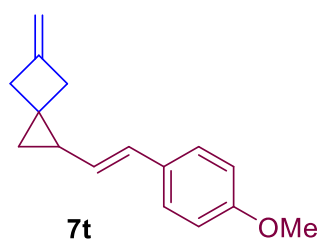

Prepared following General Procedure B, using Ni(cod $)_{2}(10 \mathrm{~mol} \%, 5.6 \mathrm{mg})$, SIMes $\cdot \mathrm{HCl}(12 \mathrm{~mol} \%, 8.3 \mathrm{mg})$, LiOMe (20 mol\%, $1.5 \mathrm{mg}),(E)-1$-(buta-1,3dien-1-yl)-4-methoxybenzene $(0.20 \mathrm{mmol}, 32 \mathrm{mg})$, and [1.1.1]propellane $(0.80$ $\mathrm{mmol})$ in toluene $(3 \mathrm{~mL})$. Purification by flash column chromatography with pentane/diethyl ether (60/1) as eluent gave $7 \mathbf{t}$ (32 $\mathrm{mg}, 71 \%$ yield) as a colourless oil. IR (film) $v_{\max } / \mathrm{cm}^{-1}: 3072,2983,2902,2831,1607,1509,1243$, 1174, 1034, 840. HRMS (APCI): Calcd. for $\mathrm{C}_{16} \mathrm{H}_{19} \mathrm{O}\left([\mathrm{M}+\mathrm{H}]^{+}\right) \mathrm{m} / \mathrm{z} 227.1430$, found $\mathrm{m} / \mathrm{z}$ 227.1430. ${ }^{1} \mathrm{H}$ NMR (400 MHz, Chloroform- $d$ ) $\delta 7.16(\mathrm{~d}, J=8.8 \mathrm{~Hz}, 2 \mathrm{H}), 6.74(\mathrm{~d}, J=8.8 \mathrm{~Hz}, 2 \mathrm{H}), 6.31(\mathrm{~d}, J=$ $15.8 \mathrm{~Hz}, 1 \mathrm{H}), 5.49(\mathrm{dd}, J=15.8,9.0 \mathrm{~Hz}, 1 \mathrm{H}), 4.79-4.71(\mathrm{~m}, 2 \mathrm{H}), 3.71(\mathrm{~s}, 3 \mathrm{H}), 2.87-2.77(\mathrm{~m}, 2 \mathrm{H})$, $2.72(\mathrm{dq}, J=15.9,2.6 \mathrm{~Hz}, 1 \mathrm{H}), 2.64(\mathrm{dq}, J=15.9,2.6 \mathrm{~Hz}, 1 \mathrm{H}), 1.52(\mathrm{td}, J=8.8,5.4 \mathrm{~Hz}, 1 \mathrm{H}), 0.93$ $(\mathrm{dd}, J=8.6,5.1 \mathrm{~Hz}, 1 \mathrm{H}), 0.59$ (t, $J=5.2 \mathrm{~Hz}, 1 \mathrm{H}) .{ }^{13} \mathrm{C}$ NMR $(100 \mathrm{MHz}$, Chloroform- $d$ ) $\delta 158.5,145.1$, $130.7,129.5,128.0,126.7,113.9,106.3,55.3,40.5,36.4,25.9,23.3,20.0$.

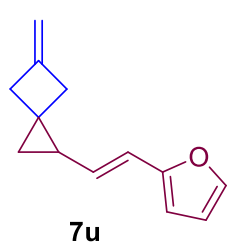

Prepared following General Procedure B, using $\mathrm{Ni}(\operatorname{cod})_{2}(10 \mathrm{~mol} \%$, $5.6 \mathrm{mg})$, SIMes $\cdot \mathrm{HCl}(12 \mathrm{~mol} \%, 8.3 \mathrm{mg}$ ), LiOMe (20 mol\%, $1.5 \mathrm{mg})$, (E)-2-(buta-1,3-dien-1yl)furan $(0.20 \mathrm{mmol}, 24 \mathrm{mg})$, and [1.1.1]propellane $(0.80 \mathrm{mmol})$ in toluene $(3 \mathrm{~mL})$. Purification by flash column chromatography with pentane as eluent gave $7 \mathbf{u}(26 \mathrm{mg}$, $70 \%$ yield) as a colourless oil. IR (film) $v_{\max } / \mathrm{cm}^{-1}: 2987,2930,2904,1677,1152$, 1012, 951, 883, 729. HRMS (APCI): Calcd. for $\mathrm{C}_{13} \mathrm{H}_{15} \mathrm{O}\left([\mathrm{M}+\mathrm{H}]^{+}\right) \mathrm{m} / \mathrm{z}$ 187.1117, found $\mathrm{m} / \mathrm{z}$ 187.1117. ${ }^{1} \mathrm{H}$ NMR (400 MHz, Chloroform- $d$ ) $\delta 7.28(\mathrm{~d}, J=1.0 \mathrm{~Hz}, 1 \mathrm{H}), 6.34(\mathrm{dd}, J=3.3$, $1.8 \mathrm{~Hz}, 1 \mathrm{H}), 6.26(\mathrm{~d}, J=15.8 \mathrm{~Hz}, 1 \mathrm{H}), 6.09(\mathrm{~d}, J=3.3 \mathrm{~Hz}, 1 \mathrm{H}), 5.68(\mathrm{dd}, J=15.8,9.3 \mathrm{~Hz}, 1 \mathrm{H}), 4.87$ $-4.81(\mathrm{~m}, 2 \mathrm{H}), 2.96-2.84(\mathrm{~m}, 2 \mathrm{H}), 2.81(\mathrm{dq}, J=15.8,2.9 \mathrm{~Hz}, 1 \mathrm{H}), 2.73(\mathrm{dq}, J=16.0,2.8 \mathrm{~Hz}, 1 \mathrm{H})$, $1.58(\mathrm{dt}, J=9.0,4.5 \mathrm{~Hz}, 1 \mathrm{H}), 1.04(\mathrm{dd}, J=8.6,5.1 \mathrm{~Hz}, 1 \mathrm{H}), 0.69(\mathrm{t}, J=5.2 \mathrm{~Hz}, 1 \mathrm{H}) .{ }^{13} \mathrm{C}$ NMR $(100$ MHz, Chloroform- $d$ ) $\delta 153.3,144.9,141.0,130.8,117.2,111.1,106.4,105.5,40.5,36.4,25.9,23.8$, 20.4 . 


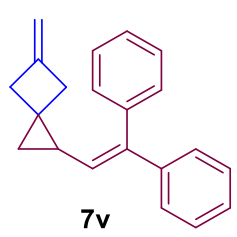

Prepared following General Procedure B, using Ni(cod) $)_{2}(10 \mathrm{~mol} \%$, $5.6 \mathrm{mg})$, SIMes $\cdot \mathrm{HCl}$ (12 mol\%, $8.3 \mathrm{mg}$ ), LiOMe (20 mol\%, $1.5 \mathrm{mg}$ ), buta-1,3-diene-1,1diyldibenzene $(0.20 \mathrm{mmol}, 42 \mathrm{mg})$, and [1.1.1]propellane $(0.80 \mathrm{mmol})$ in toluene (3 $\mathrm{mL})$. Purification by flash column chromatography with pentane as eluent gave $\mathbf{7 v}$ (42 mg, 76\% yield) as a colourless oil. IR (film) $v_{\max } / \mathrm{cm}^{-1}: 3056,3023,2985,2902$, 1677, 1494, 1444, 877, 763. HRMS (APCI): Calcd. for $\mathrm{C}_{21} \mathrm{H}_{21}\left([\mathrm{M}+\mathrm{H}]^{+}\right) \mathrm{m} / \mathrm{z}$ 273.1638, found $\mathrm{m} / \mathrm{z}$ 273.1636. ${ }^{1} \mathrm{H}$ NMR (400 MHz, Chloroform- $d$ ) $\delta 7.44-7.39(\mathrm{~m}, 2 \mathrm{H}), 7.38-$ $7.22(\mathrm{~m}, 8 \mathrm{H}), 5.46(\mathrm{~d}, J=9.8 \mathrm{~Hz}, 1 \mathrm{H}), 4.95-4.86(\mathrm{~m}, 2 \mathrm{H}), 3.06(\mathrm{dq}, J=16.6,2.9 \mathrm{~Hz}, 1 \mathrm{H}), 2.91-$ $2.72(\mathrm{~m}, 3 \mathrm{H}), 1.59(\mathrm{td}, J=9.0,5.4 \mathrm{~Hz}, 1 \mathrm{H}), 1.04(\mathrm{dd}, J=8.6,4.9 \mathrm{~Hz}, 1 \mathrm{H}), 0.74(\mathrm{t}, J=5.2 \mathrm{~Hz}, 1 \mathrm{H})$. ${ }^{13} \mathrm{C}$ NMR (100 MHz, Chloroform- $d$ ) $\delta 145.2,142.9,141.2,140.4,131.2,130.5,128.08,128.07,127.3$, $126.9,126.7,106.4,40.2,36.6,24.1,23.8,21.4$.

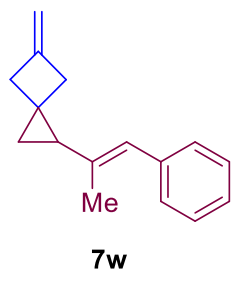

Prepared following General Procedure B, using Ni(cod) $)_{2}(10 \mathrm{~mol} \%, 5.6 \mathrm{mg})$, SIMes $\cdot \mathrm{HCl}(12 \mathrm{~mol} \%, 8.3 \mathrm{mg})$, LiOMe (20 mol\%, $1.5 \mathrm{mg}),(E)$-(2-methylbuta-1,3dien-1-yl)benzene $(0.20 \mathrm{mmol}, 29 \mathrm{mg})$, and [1.1.1]propellane $(0.80 \mathrm{mmol})$ in toluene ( $3 \mathrm{~mL}$ ). Purification by flash column chromatography with pentane as eluent gave 7w $\left(26 \mathrm{mg}, 62 \%\right.$ yield) as a colourless oil. IR (film) $v_{\max } / \mathrm{cm}^{-1}: 3059,2987,2933$, 2903, 1677, 1443, 876, 735, 698. HRMS (APCI): Calcd. for $\mathrm{C}_{16} \mathrm{H}_{19}\left([\mathrm{M}+\mathrm{H}]^{+}\right) \mathrm{m} / \mathrm{z}$ 211.1481, found $\mathrm{m} / \mathrm{z}$ 211.1481. ${ }^{1} \mathrm{H}$ NMR (400 MHz, Chloroform- $d$ ) $\delta 7.34$ (t, $J=7.5 \mathrm{~Hz}, 2 \mathrm{H}$ ), 7.26 (d, $J=7.2 \mathrm{~Hz}, 2 \mathrm{H}), 7.20(\mathrm{t}, J=7.3 \mathrm{~Hz}, 1 \mathrm{H}), 6.17(\mathrm{~s}, 1 \mathrm{H}), 4.91-4.84(\mathrm{~m}, 2 \mathrm{H}), 2.93(\mathrm{dq}, J=15.6,2.4 \mathrm{~Hz}$, $1 \mathrm{H}), 2.88-2.77(\mathrm{~m}, 2 \mathrm{H}), 2.76-2.68(\mathrm{~m}, 1 \mathrm{H}), 1.88(\mathrm{~d}, J=1.0 \mathrm{~Hz}, 3 \mathrm{H}), 1.59(\mathrm{dd}, J=8.2,6.4 \mathrm{~Hz}, 1 \mathrm{H})$, $1.01(\mathrm{t}, J=5.7 \mathrm{~Hz}, 1 \mathrm{H}), 0.92(\mathrm{dd}, J=8.6,5.4 \mathrm{~Hz}, 1 \mathrm{H}) .{ }^{13} \mathrm{C}$ NMR $(100 \mathrm{MHz}$, Chloroform- $d$ ) $\delta 145.1$, $138.5,137.5,128.9,128.0,125.8,123.9,106.1,40.9,36.4,31.5,23.3,18.3,16.6$.

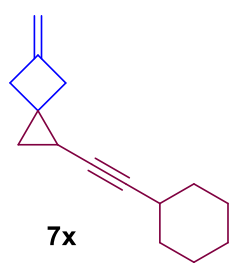

Prepared following General Procedure B, using $\mathrm{Ni}(\mathrm{cod})_{2}$ (10 mol\%, $\left.5.6 \mathrm{mg}\right)$, SIMes $\cdot \mathrm{HCl}$ (12 mol\%, $8.3 \mathrm{mg})$, LiOMe (20 mol\%, $1.5 \mathrm{mg})$, but-3-en-1-yn-1ylcyclohexane $(0.20 \mathrm{mmol}, 27 \mathrm{mg})$, and [1.1.1]propellane $(0.80 \mathrm{mmol})$ in toluene $(3$ $\mathrm{mL})$. Purification by flash column chromatography with pentane as eluent gave $\mathbf{7 x}$ (21 mg, 52\% yield) as a colourless oil. IR (film) $v_{\max } / \mathrm{cm}^{-1}: 3069,2989,2929,2850$, 1682, 1447, 874. HRMS (APCI): Calcd. for $\mathrm{C}_{15} \mathrm{H}_{21}\left([\mathrm{M}+\mathrm{H}]^{+}\right) \mathrm{m} / \mathrm{z}$ 201.1638, found m/z 201.1637. ${ }^{1} \mathrm{H}$ NMR (400 MHz, Chloroform- $d$ ) $\delta 1 \mathrm{H}$ NMR (400 MHz, Chloroform-d) $\delta 4.87$ (p, $J$ $=2.5 \mathrm{~Hz}, 1 \mathrm{H}), 4.84(\mathrm{p}, J=2.5 \mathrm{~Hz}, 1 \mathrm{H}), 3.00(\mathrm{dp}, J=17.7,2.3 \mathrm{~Hz}, 1 \mathrm{H}), 2.79-2.75(\mathrm{~m}, 2 \mathrm{H}), 2.71(\mathrm{dp}$, $J=15.1,2.1 \mathrm{~Hz}, 1 \mathrm{H}), 2.39-2.27(\mathrm{~m}, 1 \mathrm{H}), 1.80-1.63(\mathrm{~m}, 4 \mathrm{H}), 1.52-1.45(\mathrm{~m}, 1 \mathrm{H}), 1.44-1.21(\mathrm{~m}$, $6 \mathrm{H}), 0.94(\mathrm{dd}, J=8.8,4.7 \mathrm{~Hz}, 1 \mathrm{H}), 0.65(\mathrm{t}, J=5.1 \mathrm{~Hz}, 1 \mathrm{H}) \cdot{ }^{13} \mathrm{C}$ NMR $(100 \mathrm{MHz}$, Chloroform- $d$ ) $\delta$ $144.4,106.5,83.4,80.4,39.1,36.9,33.32,33.27,29.2,26.0,24.9,22.6,20.5,12.2$.

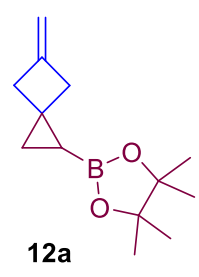

Prepared following General Procedure C, using $\mathrm{Ni}(\operatorname{cod})_{2}$ (10 mol\%, $\left.5.6 \mathrm{mg}\right)$, SIMes $\cdot \mathrm{HCl}(12 \mathrm{~mol} \%, 8.3 \mathrm{mg}), \mathrm{NaO}^{t} \mathrm{Bu}(15 \mathrm{~mol} \%, 2.9 \mathrm{mg})$, 4,4,5,5-tetramethyl-2vinyl-1,3,2-dioxaborolane $(0.20 \mathrm{mmol}, 31 \mathrm{mg})$, and [1.1.1]propellane $(0.80 \mathrm{mmol})$ in toluene ( $3 \mathrm{~mL})$. Purification by flash column chromatography with hexane/toluene $(1 / 3)$ as eluent gave 12a (30 mg, 68\% yield) as a colourless oil. IR (film) $v_{\max } / \mathrm{cm}^{-1}: 3073$, 2978, 2934, 2909, 1673, 1410, 1317, 1143, 861. HRMS (ESI): Calcd. for $\mathrm{C}_{13} \mathrm{H}_{22} \mathrm{BO}_{2}$ $\left([\mathrm{M}+\mathrm{H}]^{+}\right) \mathrm{m} / \mathrm{z}$ 221.1707, found $\mathrm{m} / \mathrm{z}$ 221.1705. ${ }^{1} \mathrm{H}$ NMR (400 MHz, Chloroform- $d$ ) $\delta$ $4.84-4.78(\mathrm{~m}, 2 \mathrm{H}), 2.95-2.84(\mathrm{~m}, 2 \mathrm{H}), 2.83-2.74(\mathrm{~m}, 2 \mathrm{H}), 1.23(\mathrm{~s}, 6 \mathrm{H}), 1.21(\mathrm{~s}, 6 \mathrm{H}), 0.87-0.76$ $(\mathrm{m}, 2 \mathrm{H}), 0.02(\mathrm{dd}, J=9.8,7.0 \mathrm{~Hz}, 1 \mathrm{H}) .{ }^{13} \mathrm{C}$ NMR (100 MHz, Chloroform- $d$ ) $\delta$ 145.6, 105.8, 83.0, $41.1,39.2,25.3,24.6,22.6,18.0$, the signal for the carbon adjacent to boron could not be observed due to quadrupolar relaxation. 


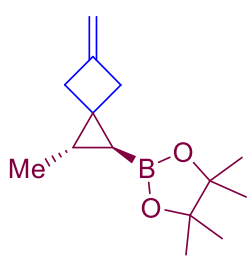

trans-12b

Prepared following General Procedure $\mathrm{C}$, using $\mathrm{Ni}(\operatorname{cod})_{2}(10 \mathrm{~mol} \%, 5.6 \mathrm{mg})$, SIMes $\cdot \mathrm{HCl}(12 \mathrm{~mol} \%, 8.3 \mathrm{mg}), \mathrm{NaO}^{t} \mathrm{Bu}(15 \mathrm{~mol} \%, 2.9 \mathrm{mg}),(E)-4,4,5,5-$ tetramethyl-2-(prop-1-en-1-yl)-1,3,2-dioxaborolane $(0.20 \mathrm{mmol}, 34 \mathrm{mg})$, and [1.1.1]propellane $(0.80 \mathrm{mmol})$ in toluene $(3 \mathrm{~mL})$. Purification by flash column chromatography with hexane/toluene (3/1) as eluent gave trans-12b $(23 \mathrm{mg}, 48 \%$ yield, >95:5 trans/cis) as a colourless oil. IR (film) $v_{\max } / \mathrm{cm}^{-1}: 3077,2978,2926$, 2909, 1679, 1440, 1361, 1316, 1144, 873, 856. HRMS (ESI): Calcd. for $\mathrm{C}_{14} \mathrm{H}_{24} \mathrm{BO}_{2}$ $\left([\mathrm{M}+\mathrm{H}]^{+}\right) \mathrm{m} / \mathrm{z}$ 235.1864, found m/z 235.1862. ${ }^{1} \mathrm{H}$ NMR (400 MHz, Chloroform- $d$ ) $\delta 4.84-4.78(\mathrm{~m}$, $2 \mathrm{H}), 2.91-2.78(\mathrm{~m}, 2 \mathrm{H}), 2.80-2.63(\mathrm{~m}, 2 \mathrm{H}), 1.23(\mathrm{~s}, 6 \mathrm{H}), 1.21(\mathrm{~s}, 6 \mathrm{H}), 1.05-0.99(\mathrm{~m}, 4 \mathrm{H}),-0.43$ $(\mathrm{d}, J=5.3 \mathrm{~Hz}, 1 \mathrm{H}) .{ }^{13} \mathrm{C}$ NMR $(100 \mathrm{MHz}$, Chloroform- $d$ ) $\delta 146.1,105.6,82.8,39.3,36.4,27.3,25.2$, 24.6, 22.5, 16.1, the signal for the carbon adjacent to boron could not be observed due to quadrupolar relaxation. ${ }^{11} \mathrm{~B}$ NMR (128 MHz, Chloroform- $d$ ) $\delta 33.8$. The trans/cis ratio was determined by GC/MS to be >95:5.

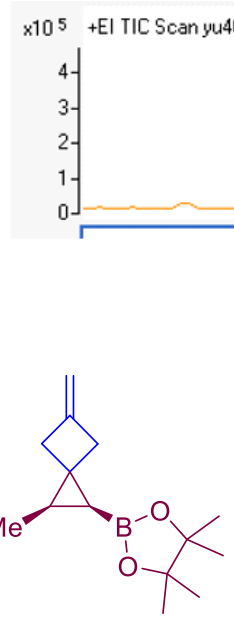

cis-12b

Prepared following General Procedure C, using Ni(cod) $2(10 \mathrm{~mol} \%, 5.6 \mathrm{mg})$, SIMes $\cdot \mathrm{HCl}(12 \mathrm{~mol} \%, 8.3 \mathrm{mg}), \mathrm{NaO}^{t} \mathrm{Bu}(15 \mathrm{~mol} \%, 2.9 \mathrm{mg}),(Z)-4,4,5,5-$ tetramethyl-2-(prop-1-en-1-yl)-1,3,2-dioxaborolane $(0.20 \mathrm{mmol}, 34 \mathrm{mg})$, and [1.1.1]propellane $(0.80 \mathrm{mmol})$ in toluene $(3 \mathrm{~mL})$. Purification by flash column chromatography with hexane/toluene (3/1) as eluent gave cis-12b $(17 \mathrm{mg}, 36 \%$ yield, 68:32 cis/trans) as a colourless oil. IR (film) $v_{\max } / \mathrm{cm}^{-1}: 2975,2921,1370$, 1316, 1144, 882. HRMS (APCI): Calcd. for $\mathrm{C}_{14} \mathrm{H}_{24} \mathrm{BO}_{2}\left([\mathrm{M}+\mathrm{H}]^{+}\right) \mathrm{m} / \mathrm{z}$ 235.1864, found $\mathrm{m} / \mathrm{z}$ 235.1860. ${ }^{1} \mathrm{H}$ NMR (400 MHz, Chloroform- $d$ ) $\delta 4.82(\mathrm{p}, J=2.4 \mathrm{~Hz}, 1 \mathrm{H}), 4.79(\mathrm{p}, J=2.5$ $\mathrm{Hz}, 1 \mathrm{H}), 2.89-2.70(\mathrm{~m}, 4 \mathrm{H}), 1.22(\mathrm{~s}, 6 \mathrm{H}), 1.21(\mathrm{~s}, 6 \mathrm{H}), 1.15-1.09(\mathrm{~m}, 1 \mathrm{H}), 1.06(\mathrm{~d}, J=5.7 \mathrm{~Hz}, 3 \mathrm{H})$, $0.04(\mathrm{~d}, J=9.4 \mathrm{~Hz}, 1 \mathrm{H}) .{ }^{13} \mathrm{C}$ NMR $(100 \mathrm{MHz}$, Chloroform- $d$ ) $\delta$ 146.2, 105.5, 82.6, 41.3, 34.7, 26.9, 25.0, 24.9, 22.8, 12.3, the signal for the carbon adjacent to boron could not be observed due to quadrupolar relaxation. ${ }^{11} \mathrm{~B}$ NMR $(128 \mathrm{MHz}$, Chloroform- $d) \delta 32.6$. The trans/cis ratio was determined by GC/MS to be 32:68.

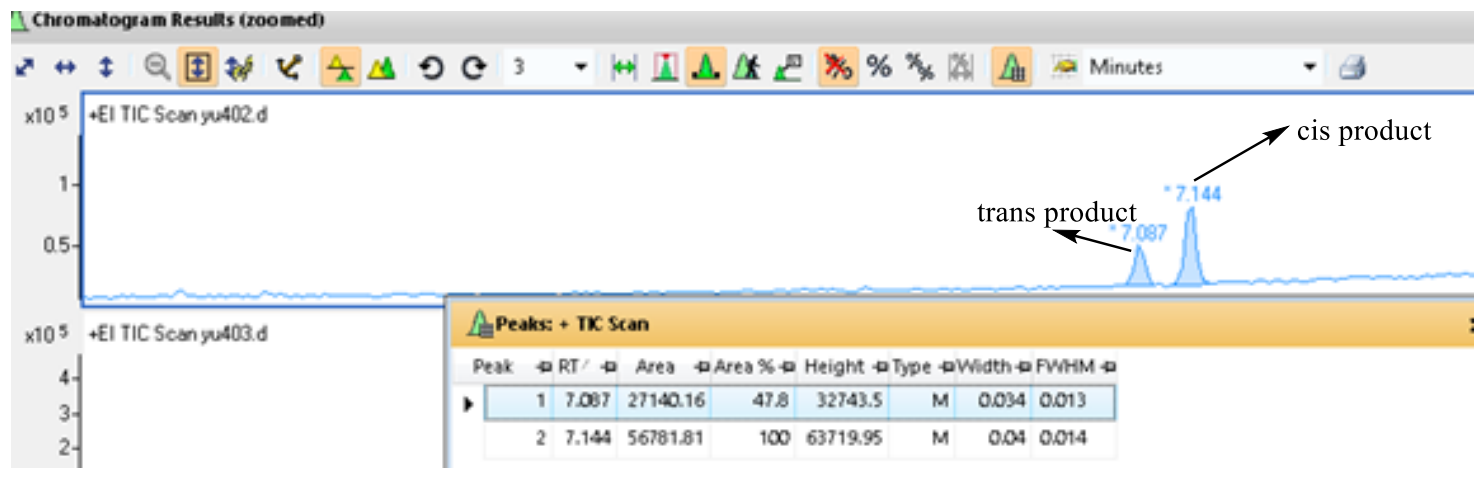




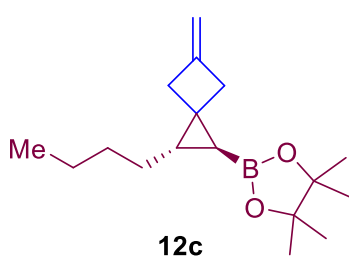

Prepared following General Procedure C, using Ni(cod $)_{2}(10 \mathrm{~mol} \%, 5.6 \mathrm{mg})$, SIMes $\cdot \mathrm{HCl}(12 \mathrm{~mol} \%, 8.3 \mathrm{mg}), \mathrm{NaO}^{t} \mathrm{Bu}(15 \mathrm{~mol} \%, 2.9 \mathrm{mg}),(E)-2-($ hex-1en-1-yl)-4,4,5,5-tetramethyl-1,3,2-dioxaborolane (0.20 mmol, $42 \mathrm{mg})$, and [1.1.1]propellane $(0.80 \mathrm{mmol})$ in toluene $(3 \mathrm{~mL})$. Purification by flash column chromatography with hexane/toluene (3/1) as eluent gave 12c (31 $\mathrm{mg}, 55 \%$ yield, $>95: 5$ trans $/$ cis ) as a colourless oil. IR (film) $\nu_{\max } / \mathrm{cm}^{-1}: 2980$, 2924, 2858, 1674, 1455, 1370, 1316, 1144, 874, 857. HRMS (APCI): Calcd. for $\mathrm{C}_{17} \mathrm{H}_{30} \mathrm{BO}_{2}\left([\mathrm{M}+\mathrm{H}]^{+}\right)$ $\mathrm{m} / \mathrm{z}$ 277.2333, found $\mathrm{m} / \mathrm{z}$ 277.2331. ${ }^{1} \mathrm{H}$ NMR (400 MHz, Chloroform- $d$ ) $\delta 4.84-4.75(\mathrm{~m}, 2 \mathrm{H}), 2.91-$ $2.65(\mathrm{~m}, 4 \mathrm{H}), 1.44-1.24(\mathrm{~m}, 6 \mathrm{H}), 1.23(\mathrm{~s}, 6 \mathrm{H}), 1.21(\mathrm{~s}, 6 \mathrm{H}), 0.99(\mathrm{q}, J=6.6 \mathrm{~Hz}, 1 \mathrm{H}), 0.89(\mathrm{t}, J=7.1$ $\mathrm{Hz}, 3 \mathrm{H}),-0.39(\mathrm{~d}, J=6.3 \mathrm{~Hz}, 1 \mathrm{H}) .{ }^{13} \mathrm{C}$ NMR $(100 \mathrm{MHz}$, Chloroform- $d$ ) $\delta 146.1,105.5,82.8,39.6$, $36.9,31.8,31.3,28.6,27.2,25.2,24.6,22.6,14.2$, the signal for the carbon adjacent to boron could not be observed due to quadrupolar relaxation. ${ }^{11} \mathrm{~B}$ NMR $(128 \mathrm{MHz}$, Chloroform- $d) \delta 32.9$.

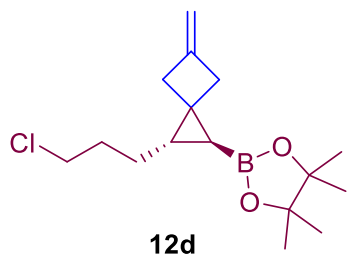

Prepared following General Procedure C, using $\mathrm{Ni}(\mathrm{cod})_{2}(10 \mathrm{~mol} \%, 5.6 \mathrm{mg})$, SIMes $\cdot \mathrm{HCl}(12 \mathrm{~mol} \%, 8.3 \mathrm{mg}), \mathrm{NaO}^{t} \mathrm{Bu}(15 \mathrm{~mol} \%, 2.9 \mathrm{mg}),(E)-2-(5-$ chloropent-1-en-1-yl)-4,4,5,5-tetramethyl-1,3,2-dioxaborolane $(0.20 \mathrm{mmol}$, $46 \mathrm{mg})$, and [1.1.1]propellane $(0.80 \mathrm{mmol})$ in toluene $(3 \mathrm{~mL})$. Purification by flash column chromatography with hexane/toluene (3/1) as eluent gave 12d (35 mg, 59\% yield, >95:5 trans/cis) as a colourless oil. IR (film) $v_{\max } / \mathrm{cm}^{-1}$ : 2978, 2930, 2911, 1677, 1448, 1389, 1370, 1317, 1143, 876, 857. HRMS (APCI): Calcd. for $\mathrm{C}_{16} \mathrm{H}_{27} \mathrm{BClO}_{2}\left([\mathrm{M}+\mathrm{H}]^{+}\right) \mathrm{m} / \mathrm{z}$ 297.1787, found $\mathrm{m} / \mathrm{z}$ 297.1778. ${ }^{1} \mathrm{H}$ NMR (400 MHz, Chloroform- $d$ ) $\delta$ $4.85-4.78(\mathrm{~m}, 2 \mathrm{H}), 3.56(\mathrm{t}, J=6.7 \mathrm{~Hz}, 2 \mathrm{H}), 2.93-2.68(\mathrm{~m}, 4 \mathrm{H}), 1.88(\mathrm{p}, J=7.1 \mathrm{~Hz}, 2 \mathrm{H}), 1.51-$ $1.39(\mathrm{~m}, 1 \mathrm{H}), 1.35-1.27(\mathrm{~m}, 1 \mathrm{H}), 1.23(\mathrm{~s}, 6 \mathrm{H}), 1.21(\mathrm{~s}, 6 \mathrm{H}), 1.00(\mathrm{dd}, J=13.1,6.3 \mathrm{~Hz}, 1 \mathrm{H}),-0.34$ $(\mathrm{d}, J=6.3 \mathrm{~Hz}, 1 \mathrm{H}) .{ }^{13} \mathrm{C}$ NMR $(100 \mathrm{MHz}$, Chloroform- $d$ ) $\delta 145.5,105.8,82.9,44.9,39.5,36.8,32.6$, $28.8,27.5,27.2,25.2,24.6$, the signal for the carbon adjacent to boron could not be observed due to quadrupolar relaxation. ${ }^{11} \mathrm{~B}$ NMR $(128 \mathrm{MHz}$, Chloroform- $d$ ) $\delta 32.7$.

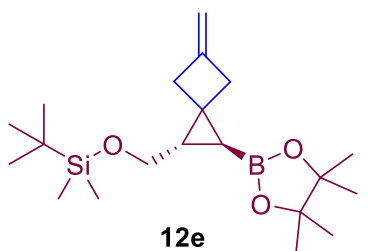

Prepared following General Procedure C, using Ni(cod $)_{2}(10 \mathrm{~mol} \%, 5.6 \mathrm{mg})$, SIMes $\cdot \mathrm{HCl}(12 \mathrm{~mol} \%, 8.3 \mathrm{mg}), \mathrm{NaO}^{t} \mathrm{Bu}(15 \mathrm{~mol} \%, 2.9 \mathrm{mg}),(E)$-tert-butyl dimethyl((3-(4,4,5,5-tetramethyl-1,3,2-dioxaborolan-2-yl)allyl)oxy)silane $(0.20 \mathrm{mmol}, 60 \mathrm{mg})$, and [1.1.1]propellane $(0.80 \mathrm{mmol})$ in toluene $(3 \mathrm{~mL})$. Purification by flash column chromatography with hexane/toluene $(2 / 1)$ as eluent gave 12e (21 mg, 28\% yield, >95:5 trans/cis) as a colourless oil. IR (film) $v_{\max } / \mathrm{cm}^{-1}: 2978,2933,2911,1380,1317,1144,874,855$. HRMS (ESI): Calcd. for $\mathrm{C}_{20} \mathrm{H}_{41} \mathrm{BNO}_{3}$ $\left(\left[\mathrm{M}+\mathrm{NH}_{4}\right]^{+}\right) \mathrm{m} / \mathrm{z}$ 382.2943, found $\mathrm{m} / \mathrm{z}$ 382.2944. ${ }^{1} \mathrm{H}$ NMR (400 MHz, Chloroform- $d$ ) $\delta 4.85-4.75$ $(\mathrm{m}, 2 \mathrm{H}), 3.64(\mathrm{dd}, J=10.9,5.8 \mathrm{~Hz}, 1 \mathrm{H}), 3.40(\mathrm{dd}, J=10.9,7.4 \mathrm{~Hz}, 1 \mathrm{H}), 2.95(\mathrm{dq}, J=15.9,2.7 \mathrm{~Hz}$, $1 \mathrm{H}), 2.89-2.78(\mathrm{~m}, 2 \mathrm{H}), 2.72(\mathrm{dq}, J=16.0,2.7 \mathrm{~Hz}, 1 \mathrm{H}), 1.31(\mathrm{dt}, J=7.4,6.1 \mathrm{~Hz}, 1 \mathrm{H}), 1.22(\mathrm{~s}, 6 \mathrm{H})$, $1.21(\mathrm{~s}, 6 \mathrm{H}), 0.88(\mathrm{~s}, 9 \mathrm{H}), 0.06(\mathrm{~s}, 3 \mathrm{H}), 0.04(\mathrm{~s}, 3 \mathrm{H}),-0.16(\mathrm{~d}, J=6.3 \mathrm{~Hz}, 1 \mathrm{H}) .{ }^{13} \mathrm{C}$ NMR $(100 \mathrm{MHz}$, Chloroform- $d$ ) $\delta 145.8,105.6,83.0,65.0,39.5,37.1,29.7,26.6,25.9,25.2,24.6,18.2,-5.0,-5.2$, the signal for the carbon adjacent to boron could not be observed due to quadrupolar relaxation. ${ }^{11} \mathrm{~B}$ NMR (128 MHz, Chloroform- $d$ ) $\delta 32.7$.

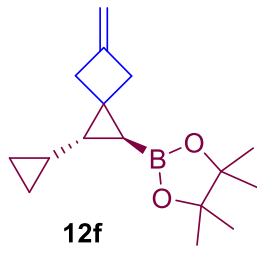

Prepared following General Procedure C, using $\mathrm{Ni}(\operatorname{cod})_{2}(10 \mathrm{~mol} \%, 5.6 \mathrm{mg})$, SIMes $\cdot \mathrm{HCl}$ (12 mol\%, $8.3 \mathrm{mg}), \mathrm{NaO}^{t} \mathrm{Bu}(15 \mathrm{~mol} \%, 2.9 \mathrm{mg}),(E)-2-(2-$ cyclopropylvinyl)-4,4,5,5-tetramethyl-1,3,2-dioxaborolane $(0.20 \mathrm{mmol}, 40 \mathrm{mg})$, and [1.1.1]propellane $(0.80 \mathrm{mmol})$ in toluene $(3 \mathrm{~mL})$. Purification by flash column chromatography with hexane/toluene (3/1) as eluent gave $\mathbf{1 2 f}$ (17 $\mathrm{mg}, 31 \%$ yield, 
$>95: 5$ trans $/$ cis) as a colourless oil. IR (film) $v_{\max } / \mathrm{cm}^{-1}: 2978,2923,1406,1308,1144,874,854$. HRMS (APCI): Calcd. for $\mathrm{C}_{16} \mathrm{H}_{26} \mathrm{BO}_{2}\left([\mathrm{M}+\mathrm{H}]^{+}\right) \mathrm{m} / \mathrm{z}$ 261.2020, found $\mathrm{m} / \mathrm{z}$ 261.2013. ${ }^{1} \mathrm{H}$ NMR (400 MHz, Chloroform- $d$ ) $\delta 4.84-4.78(\mathrm{~m}, 2 \mathrm{H}), 2.89-2.69(\mathrm{~m}, 4 \mathrm{H}), 1.21(\mathrm{~s}, 6 \mathrm{H}), 1.20(\mathrm{~s}, 6 \mathrm{H}), 0.92(\mathrm{t}, J$ $=6.2 \mathrm{~Hz}, 1 \mathrm{H}), 0.58-0.44(\mathrm{~m}, 2 \mathrm{H}), 0.37-0.29(\mathrm{~m}, 1 \mathrm{H}), 0.17-0.10(\mathrm{~m}, 1 \mathrm{H}), 0.09-0.03(\mathrm{~m}, 1 \mathrm{H}),-$ $0.32(\mathrm{~d}, J=6.3 \mathrm{~Hz}, 1 \mathrm{H}) .{ }^{13} \mathrm{C}$ NMR $\left(100 \mathrm{MHz}, \mathrm{CDCl}_{3}\right) \delta 146.3,105.6,82.9,39.7,37.4,31.4,27.2$, $25.2,24.6,10.9,3.9,2.6$, the signal for the carbon adjacent to boron could not be observed due to quadrupolar relaxation. ${ }^{11} \mathrm{~B} \mathrm{NMR}\left(128 \mathrm{MHz}, \mathrm{CDCl}_{3}\right) \delta 33.0$.

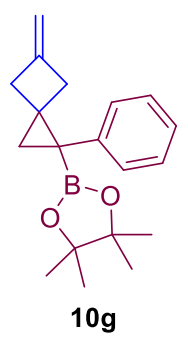

Prepared following General Procedure C, using Ni(cod $)_{2}(10 \mathrm{~mol} \%, 5.6 \mathrm{mg})$, SIMes $\cdot \mathrm{HCl}$ (12 mol\%, $8.3 \mathrm{mg}$ ), NaO${ }^{t} \mathrm{Bu}(15 \mathrm{~mol} \%, 2.9 \mathrm{mg}$ ), 4,4,5,5-tetramethyl-2-(1-phenylvinyl)1,3,2-dioxaborolane $(0.20 \mathrm{mmol}, 46 \mathrm{mg})$, and [1.1.1]propellane $(0.80 \mathrm{mmol})$ in toluene (3 mL). Purification by flash column chromatography with hexane/toluene $(2 / 1)$ as eluent gave $12 \mathrm{~g}$ (42 mg, 71\% yield) as a colourless oil. IR (film) $v_{\max } / \mathrm{cm}^{-1}: 3058,2979$, 2906, 1677, 1380, 1307, 1142, 853, 699. HRMS (APCI): Calcd. for $\mathrm{C}_{19} \mathrm{H}_{26} \mathrm{BO}_{2}$ $\left([\mathrm{M}+\mathrm{H}]^{+}\right) \mathrm{m} / \mathrm{z}$ 297.2020, found $\mathrm{m} / \mathrm{z}$ 297.2018. ${ }^{1} \mathrm{H}$ NMR (400 MHz, Chloroform- $d$ ) $\delta$ $7.28-7.17(\mathrm{~m}, 4 \mathrm{H}), 7.12(\mathrm{td}, J=6.9,1.6 \mathrm{~Hz}, 1 \mathrm{H}), 4.81(\mathrm{p}, J=2.4 \mathrm{~Hz}, 1 \mathrm{H}), 4.75(\mathrm{p}, J=$ $2.5 \mathrm{~Hz}, 1 \mathrm{H}), 3.07$ (dq, $J=16.5,3.0 \mathrm{~Hz}, 1 \mathrm{H}), 2.89-2.79(\mathrm{~m}, 1 \mathrm{H}), 2.49$ (dq, $J=16.4,3.0 \mathrm{~Hz}, 1 \mathrm{H}), 2.32$ $-2.22(\mathrm{~m}, 1 \mathrm{H}), 1.39(\mathrm{~d}, J=4.3 \mathrm{~Hz}, 1 \mathrm{H}), 1.28(\mathrm{~d}, J=4.3 \mathrm{~Hz}, 1 \mathrm{H}), 1.22(\mathrm{~s}, 6 \mathrm{H}), 1.18(\mathrm{~s}, 6 \mathrm{H}) .{ }^{13} \mathrm{C}$ NMR $(100 \mathrm{MHz}$, Chloroform- $d$ ) $\delta 145.1,141.7,129.4,127.8,125.2,105.7,83.4,40.2,37.5,29.0,24.92$, $24.88,23.1$, the signal for the carbon adjacent to boron could not be observed due to quadrupolar relaxation. ${ }^{11} \mathrm{~B}$ NMR $(128 \mathrm{MHz}$, Chloroform- $d$ ) $\delta 32.8$.

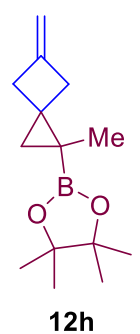

Prepared following General Procedure C, using Ni(cod $)_{2}(10 \mathrm{~mol} \%, 5.6 \mathrm{mg})$, SIMes $\cdot \mathrm{HCl}$ (12 mol\%, $8.3 \mathrm{mg}$ ), $\mathrm{NaO}^{t} \mathrm{Bu}(15 \mathrm{~mol} \%, 2.9 \mathrm{mg}$ ), 4,4,5,5-tetramethyl-2-(prop-1-en-2-yl)1,3,2-dioxaborolane $(0.20 \mathrm{mmol}, 34 \mathrm{mg})$, and [1.1.1]propellane $(0.80 \mathrm{mmol})$ in toluene $(3$ $\mathrm{mL})$. Purification by flash column chromatography with hexane/toluene $(2 / 1)$ as eluent gave 12h (30 mg, 64\% yield) as a colourless oil. IR (film) $v_{\max } / \mathrm{cm}^{-1}: 2979,2905,1677$, 1462, 1401, 1304, 1145, 1133, 874, 855, 690. HRMS (APCI): Calcd. for $\mathrm{C}_{14} \mathrm{H}_{24} \mathrm{BO}_{2}$ $\left([\mathrm{M}+\mathrm{H}]^{+}\right) \mathrm{m} / \mathrm{z} 235.1864$, found $\mathrm{m} / \mathrm{z}$ 235.1863. ${ }^{1} \mathrm{H}$ NMR (400 MHz, Chloroform- $d$ ) $\delta 4.85-$ $4.78(\mathrm{~m}, 2 \mathrm{H}), 2.95(\mathrm{dq}, J=16.0,3.0 \mathrm{~Hz}, 1 \mathrm{H}), 2.87(\mathrm{dq}, J=16.3,3.0 \mathrm{~Hz}, 1 \mathrm{H}), 2.71(\mathrm{dq}, J=$ 16.3, $2.9 \mathrm{~Hz}, 1 \mathrm{H}), 2.57(\mathrm{dq}, J=16.0,2.9 \mathrm{~Hz}, 1 \mathrm{H}), 1.22(\mathrm{~s}, 6 \mathrm{H}), 1.20(\mathrm{~s}, 6 \mathrm{H}), 0.96(\mathrm{~d}, J=3.7 \mathrm{~Hz}, 1 \mathrm{H})$, $0.95(\mathrm{~s}, 3 \mathrm{H}), 0.32(\mathrm{~d}, J=3.7 \mathrm{~Hz}, 1 \mathrm{H}) .{ }^{13} \mathrm{C}$ NMR $(100 \mathrm{MHz}$, Chloroform- $d$ ) $\delta$ 145.7, 105.6, 83.0, 39.7, $36.5,26.7,25.4,25.2,24.6,17.3$, the signal for the carbon adjacent to boron could not be observed due to quadrupolar relaxation. ${ }^{11} \mathrm{~B}$ NMR $(128 \mathrm{MHz}$, Chloroform- $d$ ) $\delta 33.4$.

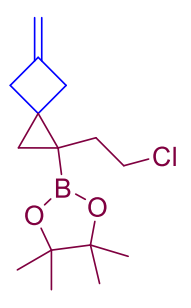

Prepared following General Procedure C, using $\mathrm{Ni}(\operatorname{cod})_{2}(10 \mathrm{~mol} \%$, $5.6 \mathrm{mg})$, SIMes $\cdot \mathrm{HCl}(12 \mathrm{~mol} \%, 8.3 \mathrm{mg}), \mathrm{NaO}^{t} \mathrm{Bu}(15 \mathrm{~mol} \%, 2.9 \mathrm{mg}), 2$-(4-chlorobut-1-en-2-yl)4,4,5,5-tetramethyl-1,3,2-dioxaborolane $(0.20 \mathrm{mmol}, 44 \mathrm{mg})$, and [1.1.1]propellane $(0.80 \mathrm{mmol})$ in toluene $(3 \mathrm{~mL})$. Purification by flash column chromatography with hexane/toluene (2/1) as eluent gave $\mathbf{1 2 i}$ ( $32 \mathrm{mg}, 57 \%$ yield) as a colourless oil. IR (film) $v_{\max } / \mathrm{cm}^{-1}:$ 2979, 2929, 2903, 1406, 1308, 1143, 877, 856. HRMS (APCI): Calcd. for $\mathrm{C}_{15} \mathrm{H}_{25} \mathrm{BClO}_{2}\left([\mathrm{M}+\mathrm{H}]^{+}\right) \mathrm{m} / \mathrm{z}$ 283.1631, found $\mathrm{m} / \mathrm{z}$ 283.1622. ${ }^{1} \mathrm{H}$ NMR $(400 \mathrm{MHz}$, Chloroform- $d$ ) $\delta 4.86-4.79(\mathrm{~m}, 2 \mathrm{H}), 3.66(\mathrm{td}, J=10.2,5.7 \mathrm{~Hz}, 1 \mathrm{H}), 3.58(\mathrm{td}, J=10.1$, $5.8 \mathrm{~Hz}, 1 \mathrm{H}), 2.97(\mathrm{dq}, J=15.9,3.0 \mathrm{~Hz}, 1 \mathrm{H}), 2.87(\mathrm{dq}, J=16.3,3.0 \mathrm{~Hz}, 1 \mathrm{H}), 2.76-2.67(\mathrm{~m}, 1 \mathrm{H}), 2.62$ $(\mathrm{dq}, J=16.1,3.4 \mathrm{~Hz}, 1 \mathrm{H}), 2.06(\mathrm{ddd}, J=13.4,10.0,5.7 \mathrm{~Hz}, 1 \mathrm{H}), 1.26-1.19(\mathrm{~m}, 1 \mathrm{H}), 1.21(\mathrm{~s}, 6 \mathrm{H})$, $1.19(\mathrm{~s}, 6 \mathrm{H}), 1.03(\mathrm{~d}, J=4.0 \mathrm{~Hz}, 1 \mathrm{H}), 0.48(\mathrm{~d}, J=4.0 \mathrm{~Hz}, 1 \mathrm{H}) .{ }^{13} \mathrm{C}$ NMR $(100 \mathrm{MHz}$, Chloroform- $d) \delta$ 

$\delta 32.9$.

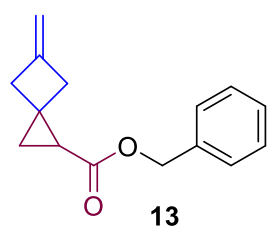

Prepared following General Procedure B, using Ni(cod) $)_{2}(10 \mathrm{~mol} \%$, $5.6 \mathrm{mg})$, SIMes $\cdot \mathrm{HCl}(12 \mathrm{~mol} \%, 8.3 \mathrm{mg})$, LiOMe $(20 \mathrm{~mol} \%, 1.5 \mathrm{mg})$, benzyl acrylate $(0.20$ $\mathrm{mmol}, 33 \mathrm{mg})$, and [1.1.1]propellane $(0.80 \mathrm{mmol})$ in toluene $(3 \mathrm{~mL})$. Purification by flash column chromatography with toluene as eluent gave $\mathbf{1 3}(18 \mathrm{mg}, 40 \%$ yield) as a colourless oil. IR (film) $v_{\max } / \mathrm{cm}^{-1}: 3069,2987,2910,1721,1397,1313$, 1168, 1142, 879, 698. HRMS (APCI): Calcd. for $\mathrm{C}_{15} \mathrm{H}_{17} \mathrm{O}_{2}\left([\mathrm{M}+\mathrm{H}]^{+}\right) \mathrm{m} / \mathrm{z}$ 229.1223, found $\mathrm{m} / \mathrm{z}$ 229.1219. ${ }^{1} \mathrm{H}$ NMR $(400 \mathrm{MHz}$, Chloroform- $d$ ) $\delta 7.38-7.30(\mathrm{~m}, 5 \mathrm{H}), 5.14(\mathrm{~d}, J$ $=12.5 \mathrm{~Hz}, 1 \mathrm{H}), 5.10(\mathrm{~d}, J=12.3 \mathrm{~Hz}, 1 \mathrm{H}), 4.89-4.81(\mathrm{~m}, 2 \mathrm{H}), 2.98-2.75(\mathrm{~m}, 4 \mathrm{H}), 1.75(\mathrm{dd}, J=8.5$, $5.4 \mathrm{~Hz}, 1 \mathrm{H}), 1.29(\mathrm{t}, J=5.1 \mathrm{~Hz}, 1 \mathrm{H}), 1.13(\mathrm{dd}, J=8.5,4.7 \mathrm{~Hz}, 1 \mathrm{H}) .{ }^{13} \mathrm{C}$ NMR $\left(100 \mathrm{MHz}, \mathrm{CDCl}_{3}\right) \delta$ $172.6,143.6,136.2,128.5,128.2,128.2,106.8,66.2,39.7,37.6,26.3,24.7,20.5$. 


\section{Product Derivatization}

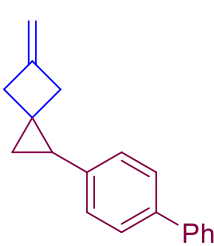

$7 a$

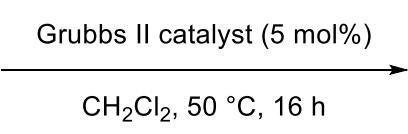

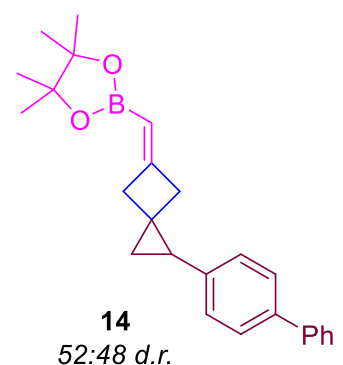

To a flame-dried pressure tube was added a solution of Grubb's $2^{\text {nd }}$ generation catalyst $(5 \mathrm{~mol} \%, 6.4$ $\mathrm{mg}$ ) in $\mathrm{CH}_{2} \mathrm{Cl}_{2}(3 \mathrm{~mL})$, followed by (E)-4,4,5,5-tetramethyl-2-(prop-1-en-1-yl)-1,3,2-dioxaborolane $(0.15 \mathrm{mmol}, 25.2 \mathrm{mg})$ and 1-([1,1'-biphenyl]-4-yl)-5-methylenespiro[2.3]hexane (7a, $0.15 \mathrm{mmol}, 36.9$ $\mathrm{mg}$ ). The solution was refluxed at $50{ }^{\circ} \mathrm{C}$ for $16 \mathrm{~h}$. The mixture was then concentrated under reduced pressure and the residue was purified by silica gel flash column chromatography with petane/diethyl ether (30/1) as eluent to provide $\mathbf{1 4}$ in $69 \%$ yield as a colourless oil. The diastereomeric ratio was determined by ${ }^{1} \mathrm{H}$ NMR to be 52:48. IR (film) $v_{\max } / \mathrm{cm}^{-1}: 2977,2901,1669,1361,1317,1242,1142$, 848, 763, 697. HRMS (APCI): Calcd. for $\mathrm{C}_{25} \mathrm{H}_{30} \mathrm{O}_{2} \mathrm{~B}\left([\mathrm{M}+\mathrm{H}]^{+}\right) \mathrm{m} / \mathrm{z} 373.2333$, found $\mathrm{m} / \mathrm{z}$ 373.2334. ${ }^{1} \mathrm{H}$ NMR (400 MHz, Chloroform- $d$ ) $\delta(52: 48$ mixture of diastereomers) $7.63-7.56(\mathrm{~m}, 2 \mathrm{H}), 7.56-$ $7.49(\mathrm{~m}, 2 \mathrm{H}), 7.47-7.40(\mathrm{~m}, 2 \mathrm{H}), 7.36-7.30(\mathrm{~m}, 1 \mathrm{H}), 7.11-7.01(\mathrm{~m}, 2 \mathrm{H}), 5.25(\mathrm{p}, J=2.4 \mathrm{~Hz}$, $0.52 \mathrm{H}), 5.22(\mathrm{p}, J=2.4 \mathrm{~Hz}, 0.48 \mathrm{H}), 3.21-2.70(\mathrm{~m}, 4 \mathrm{H}), 2.09-1.99(\mathrm{~m}, 1 \mathrm{H}), 1.26+1.23+1.21$ (diastereomeric peaks, $3 \times \mathrm{s}, 12 \mathrm{H}), 1.03(\mathrm{t}, J=5.4 \mathrm{~Hz}, 0.52 \mathrm{H}), 1.02(\mathrm{t}, J=5.4 \mathrm{~Hz}, 0.48 \mathrm{H}) .{ }^{13} \mathrm{C} \mathrm{NMR}$ $(100 \mathrm{MHz}$, Chloroform- $d$ ) $\delta$ (mixture of diastereomers) Major: 163.3, 141.1, 140.3, 138.1, 128.72 , 127.1, 126.93, 126.90, 126.82, 82.6, 42.9, 38.4, 27.4, 25.0, 24.89, 24.86, 24.9, 20.0. Minor: 163.5, 141.06, 140.2, 138.2, 128.70, 127.0, 126.91, 126.79, 43.3, 38.7, 24.92, 24.82, 19.7 (four signals are missing due to overlap). The signal for the carbon adjacent to boron could not be observed due to quadrupolar relaxation. ${ }^{11} \mathrm{~B}$ NMR $(128 \mathrm{MHz}$, Chloroform- $d) \delta 29.5$.

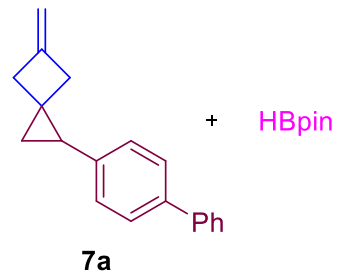

$7 a$

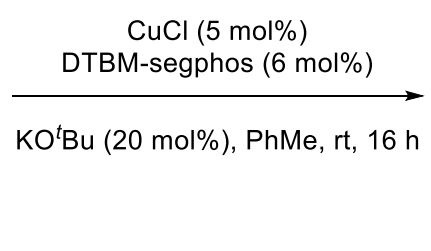

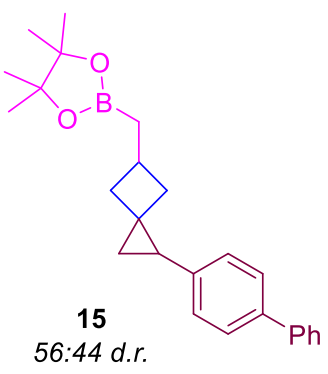

A mixture of $\mathrm{CuCl}(5 \mathrm{~mol} \%, 0.74 \mathrm{mg})$, DTBM-Segphos (6 mol\%, $10.6 \mathrm{mg})$ and $\mathrm{KO}^{t} \mathrm{Bu}(20 \mathrm{~mol} \%, 3.5$ $\mathrm{mg})$ in toluene $(2 \mathrm{~mL})$ was stirred for $5 \mathrm{~min}$ in a Schlenk tube under an $\mathrm{N}_{2}$ atmosphere. HBpin (1.2 equiv, $0.18 \mathrm{mmol}, 23.0 \mathrm{mg})$ was added to the reaction mixture and stirred for $15 \mathrm{~min}$ at rt. 1-([1,1'biphenyl]-4-yl)-5-methylenespiro[2.3] hexane (7a, $0.15 \mathrm{mmol}, 36.9 \mathrm{mg}$ ) was added and the mixture was stirred at $\mathrm{rt}$ for $12 \mathrm{~h}$. The reaction mixture was purified directly by silica gel flash column chromatography using pentane/diethyl ether (30/1) as eluent to provide $\mathbf{1 5}$ in $89 \%$ yield as a colourless oil. The diastereomeric ratio was determined by ${ }^{1} \mathrm{H}$ NMR to be $56: 44$. IR (film) $v_{\max } / \mathrm{cm}^{-1}$ : 3028, 2977, 2918, 2847, 1487, 1368, 1313, 1143, 846, 763, 699. HRMS (APCI): Calcd. for $\mathrm{C}_{25} \mathrm{H}_{32} \mathrm{O}_{2} \mathrm{~B}\left([\mathrm{M}+\mathrm{H}]^{+}\right) \mathrm{m} / \mathrm{z}$ 375.2490, found $\mathrm{m} / \mathrm{z}$ 375.2490. ${ }^{1} \mathrm{H}$ NMR (400 MHz, Chloroform- $d$ ) $\delta$ (56:44 mixture of diastereomers) $7.62-7.57(\mathrm{~m}, 2 \mathrm{H}), 7.53-7.48(\mathrm{~m}, 2 \mathrm{H}), 7.46-7.40(\mathrm{~m}, 2 \mathrm{H}), 7.35$ $-7.29(\mathrm{~m}, 1 \mathrm{H}), 7.07-7.02(\mathrm{~m}, 2 \mathrm{H}), 2.74-2.62(\mathrm{~m}, 0.56 \mathrm{H}), 2.55-2.44(\mathrm{~m}, 0.44 \mathrm{H}), 2.41-2.09(\mathrm{~m}$, $2 \mathrm{H}), 2.01-1.81(\mathrm{~m}, 2 \mathrm{H}), 1.79-1.68(\mathrm{~m}, 1 \mathrm{H}), 1.24+1.22$ (diastereomeric peaks, $2 \times \mathrm{s}, 12 \mathrm{H}), 1.14-$ 
$1.06(\mathrm{~m}, 1 \mathrm{H}), 1.12(\mathrm{~d}, J=7.5,0.88 \mathrm{H}), 1.00(\mathrm{~d}, J=7.8 \mathrm{~Hz}, 1.12 \mathrm{H}), 0.92(\mathrm{t}, J=5.5 \mathrm{~Hz}, 0.56 \mathrm{H}), 0.87(\mathrm{t}$, $J=5.6 \mathrm{~Hz}, 0.44 \mathrm{H}) .{ }^{13} \mathrm{C}$ NMR $(100 \mathrm{MHz}$, Chloroform- $d$ ) $\delta$ (mixture of diastereomers) Major: 141.24 , $140.88,137.77,128.68,127.2$, 126.89, 126.82, 126.69, 82.86, 39.75, 35.49, 27.21, 26.58, 25.62, 24.82, 24.79, 20.97; Minor: 141.20, 140.89, 137.83, 128.69, 126.85, 126.64, 82.89, 39.91, 34.33, $27.62,26.81,25.68,24.85,20.11$ (three signals are missing due to overlap). The signal for the carbon adjacent to boron could not be observed due to quadrupolar relaxation. ${ }^{11} \mathrm{~B} \mathrm{NMR}(128 \mathrm{MHz}$, Chloroform- $d$ ) $\delta 33.6$.
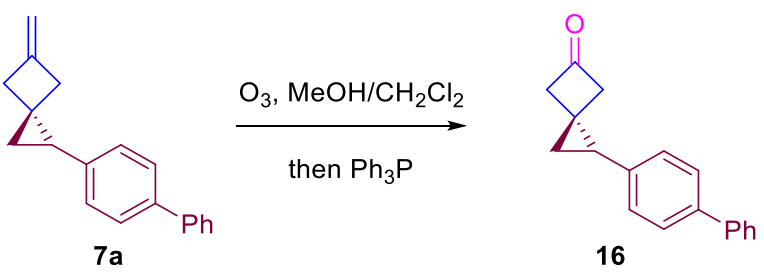

1-([1,1'-Biphenyl]-4-yl)-5-methylenespiro[2.3]hexane (7a, $0.15 \mathrm{mmol}, 36.9 \mathrm{mg})$ was dissolved in $\mathrm{MeOH} / \mathrm{CH}_{2} \mathrm{Cl}_{2}(1: 5,3 \mathrm{~mL})$ and cooled to $-78{ }^{\circ} \mathrm{C}$. Ozone from an ozone generator was bubbled through the solution while stirring for $10 \mathrm{~min}$ with sudan red as an indicator until the red colour disappeared. $\mathrm{Ph}_{3} \mathrm{P}(1.5$ equiv, $59.0 \mathrm{mg}$ ) was added and the mixture was stirred for $2 \mathrm{~h}$ at $\mathrm{rt}$. The reaction mixture was washed with $\mathrm{H}_{2} \mathrm{O}$ and dried over $\mathrm{Na}_{2} \mathrm{SO}_{4}$, filtered and concentrated under reduced pressure. Purification by silica gel flash column chromatography using pentane/diethyl ether (20/1) as eluent provided 16 in $91 \%$ yield as a white solid. HRMS (APCI): Calcd. for $\mathrm{C}_{18} \mathrm{H}_{17} \mathrm{O}$ $\left([\mathrm{M}+\mathrm{H}]^{+}\right) \mathrm{m} / \mathrm{z}$ 249.1274, found $\mathrm{m} / \mathrm{z}$ 249.1267. IR (film) $v_{\max } / \mathrm{cm}^{-1}: 3028,2931,2903,1677,1488,876$, 761, 697. ${ }^{1} \mathrm{H}$ NMR (400 MHz, Chloroform- $d$ ) $\delta 7.63-7.52(\mathrm{~m}, 4 \mathrm{H}), 7.48-7.41(\mathrm{~m}, 2 \mathrm{H}), 7.38-7.32$ $(\mathrm{m}, 1 \mathrm{H}), 7.13-7.06(\mathrm{~m}, 2 \mathrm{H}), 3.32(\mathrm{dt}, J=18.2,2.8 \mathrm{~Hz}, 1 \mathrm{H}), 3.23(\mathrm{dt}, J=18.1,2.8 \mathrm{~Hz}, 1 \mathrm{H}), 3.08$ (dt, $J=18.5,2.9 \mathrm{~Hz}, 1 \mathrm{H}), 2.94(\mathrm{dt}, J=18.2,2.7 \mathrm{~Hz}, 1 \mathrm{H}), 2.33(\mathrm{dd}, J=8.9,6.0 \mathrm{~Hz}, 1 \mathrm{H}), 1.46(\mathrm{dd}, J=8.9$, $5.6 \mathrm{~Hz}, 1 \mathrm{H}), 1.27(\mathrm{t}, J=5.8 \mathrm{~Hz}, 1 \mathrm{H}) .{ }^{13} \mathrm{C}$ NMR (100 MHz, Chloroform- $d$ ) $\delta$ 206.0, 140.8, 139.0, $138.9,128.8,127.2,127.1,126.9,54.9,50.8,26.8,19.9,18.4$.
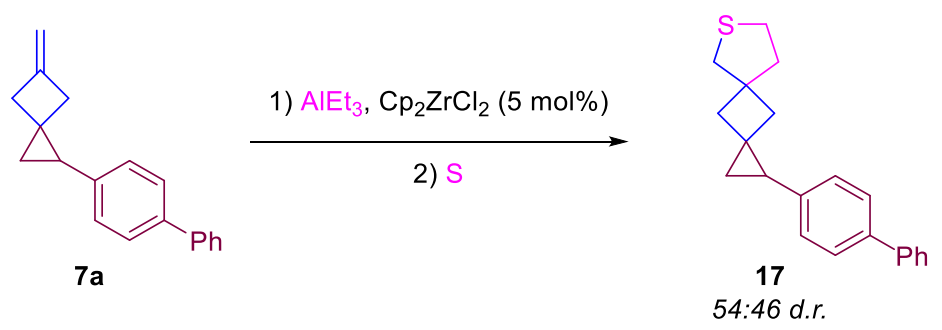

1-([1,1'-Biphenyl]-4-yl)-5-methylenespiro[2.3]hexane (7a, $0.15 \mathrm{mmol}, 36.9 \mathrm{mg}), \mathrm{Cp}_{2} \mathrm{ZrCl}_{2}$ (5 mol\%, $3 \mathrm{mg})$, and $\mathrm{Et}_{3} \mathrm{Al}(0.18 \mathrm{mmol}, 1 \mathrm{M}$ in hexane) were dissolved in hexane $(2 \mathrm{~mL})$ in a pressure tube under an $\mathrm{N}_{2}$ atmosphere at $0{ }^{\circ} \mathrm{C}$. The reaction was allowed to warm to $\mathrm{rt}$ and stirred for $4 \mathrm{~h}$ before cooling to $0{ }^{\circ} \mathrm{C}$. Sulfur powder $(0.36 \mathrm{mmol}, 11.5 \mathrm{mg})$ in toluene $(3 \mathrm{~mL})$ was added before heating the reaction to reflux for $8 \mathrm{~h}$. After allowing to cool to RT, the reaction was quenched with $7-10 \%$ aq. $\mathrm{HCl}$ and extracted with hexane, dried over $\mathrm{Na}_{2} \mathrm{SO}_{4}$, filtered and concentrated under reduced pressure. Purification by silica gel flash column chromatography using pentane/diethyl ether (100/1) as eluent provided 17 in $43 \%$ yield as a colourless oil. The diastereomeric ratio was determined by ${ }^{1} \mathrm{H}$ NMR to be 54:46. IR (film) $v_{\max } / \mathrm{cm}^{-1}: 3028,2915,2848,1487,833,763,732,697$. HRMS (APCI): Calcd. for $\mathrm{C}_{21} \mathrm{H}_{23} \mathrm{~S}\left([\mathrm{M}+\mathrm{H}]^{+}\right) \mathrm{m} / \mathrm{z} 307.1515$, found $\mathrm{m} / \mathrm{z}$ 307.1514. ${ }^{1} \mathrm{H}$ NMR $(400 \mathrm{MHz}$, Chloroform- $d$ ) $\delta(54: 46$ mixture of diastereomers) $7.62-7.55(\mathrm{~m}, 2 \mathrm{H}), 7.53-7.48(\mathrm{~m}, 2 \mathrm{H}), 7.46-7.39(\mathrm{~m}, 2 \mathrm{H}), 7.35-7.29$ $(\mathrm{m}, 1 \mathrm{H}), 7.06-7.00(\mathrm{~m}, 2 \mathrm{H}), 2.92(\mathrm{~s}, 0.92 \mathrm{H}), 2.85(\mathrm{t}, J=6.7 \mathrm{~Hz}, 1.08 \mathrm{H}), 2.81-2.69(\mathrm{~m}, 2 \mathrm{H}), 2.27-$ 
$2.10(\mathrm{~m}, 2 \mathrm{H}), 2.10-1.81(\mathrm{~m}, 5 \mathrm{H}), 1.17(\mathrm{dd}, J=9.0,5.4, \mathrm{~Hz}, 0.46 \mathrm{H}), 1.17(\mathrm{dd}, J=9.0,5.3, \mathrm{~Hz}$, $0.54 \mathrm{H}), 0.98(\mathrm{t}, J=5.6 \mathrm{~Hz}, 1 \mathrm{H}) .{ }^{13} \mathrm{C}$ NMR $(100 \mathrm{MHz}$, Chloroform- $d$ ) $\delta$ (mixture of diastereomers) Major: $141.04,140.04,138.18,128.70,126.94,126.92$, 126.87, 126.83, 45.21, 42.81, 42.00, 40.93, 36.11, 29.15, 27.45, 23.77, 20.22. Minor: 141.00, 140.10, 138.12, 128.71, 126.96, 126.91, 126.79, $45.29,43.07,41.71,40.81,35.96,29.05,27.56,23.70,20.08$ (one signal is missing due to overlap).

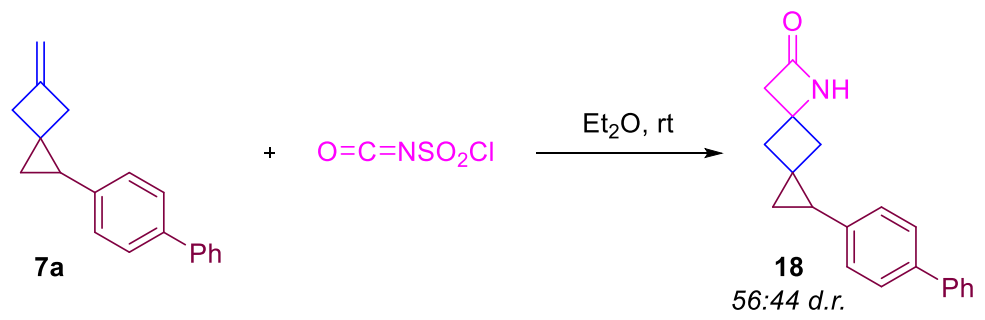

A solution of 1-([1,1'-biphenyl]-4-yl)-5-methylenespiro[2.3]hexane (7a, $0.12 \mathrm{mmol}, 30.0 \mathrm{mg})$ in $\mathrm{Et}_{2} \mathrm{O}$ $(2 \mathrm{~mL})$ at $\mathrm{rt}$ was treated with chlorosulfonyl isocyanate $(0.12 \mathrm{mmol}, 17.0 \mathrm{mg})$. The mixture was stirred at $\mathrm{rt}$ for $3 \mathrm{~h}$. The reaction mixture was then poured into an ice-cold buffer solution $(10 \mathrm{~mL})$ containing $\mathrm{Na}_{2} \mathrm{SO}_{3}(0.2 \mathrm{mmol}, 25.2 \mathrm{mg})$ and $\mathrm{Na}_{2} \mathrm{HPO}_{4}(0.2 \mathrm{mmol}, 28.4 \mathrm{mg})$. The mixture was stirred at rt overnight and then extracted with $\mathrm{CH}_{2} \mathrm{Cl}_{2}$. The combined organic layers were washed with brine, dried over $\mathrm{Na}_{2} \mathrm{SO}_{4}$, filtered and concentrated under reduced pressure. Purification by silica gel flash column chromatography with diethyl ether as eluent provided $\mathbf{1 8}$ in $41 \%$ yield as a white solid. The diastereomeric ratio was determined by ${ }^{1} \mathrm{H}$ NMR to be 56:44. HRMS (APCI): Calcd. for $\mathrm{C}_{20} \mathrm{H}_{20} \mathrm{ON}$ $\left([\mathrm{M}+\mathrm{H}]^{+}\right) \mathrm{m} / \mathrm{z}$ 290.1539, found $\mathrm{m} / \mathrm{z}$ 290.1529. ${ }^{1} \mathrm{H}$ NMR (400 MHz, Chloroform- $d$ ) $\delta(56: 44$ mixture of diastereomers) $7.61-7.55(\mathrm{~m}, 2 \mathrm{H}), 7.54-7.49(\mathrm{~m}, 2 \mathrm{H}), 7.46-7.40(\mathrm{~m}, 2 \mathrm{H}), 7.36-7.30(\mathrm{~m}, 1 \mathrm{H})$, $7.07-7.00$ (m, 2H), 6.12 (br. s, 0.56H), 5.99 (br. s, 0.44H), $3.17-3.09$ (m, 0.88H), 3.02 (dd, $J=$ $14.7,1.7 \mathrm{~Hz}, 0.56 \mathrm{H}), 2.88(\mathrm{dd}, J=14.7,1.7 \mathrm{~Hz}, 0.56 \mathrm{H}), 2.78-2.67(\mathrm{~m}, 1 \mathrm{H}), 2.54-2.41(\mathrm{~m}, 2 \mathrm{H})$, $2.40-2.35(\mathrm{~m}, 0.44 \mathrm{H}), 2.20-2.14(\mathrm{~m}, 0.56 \mathrm{H}), 2.05(\mathrm{dd}, J=8.9,6.0 \mathrm{~Hz}, 0.56 \mathrm{H}), 1.99(\mathrm{dd}, J=9.0$, $5.9 \mathrm{~Hz}, 0.44 \mathrm{H}), 1.23(\mathrm{dd}, J=9.0,5.3 \mathrm{~Hz}, 0.44 \mathrm{H}), 1.16(\mathrm{dd}, J=8.9,5.7 \mathrm{~Hz}, 0.56 \mathrm{H}), 1.04(\mathrm{t}, J=5.6 \mathrm{~Hz}$, $0.44 \mathrm{H}), 1.01(\mathrm{t}, J=5.8 \mathrm{~Hz}, 0.56 \mathrm{H}) .{ }^{13} \mathrm{C}$ NMR $(100 \mathrm{MHz}$, Chloroform- $d$ ) $\delta$ (mixture of diastereomers) Major: 166.9,0 140.84, 139.15, 138.61, 128.75, 127.18, 126.98, 126.90, 126.89, 52.52, 50.51, 43.19, 37.90, 27.29, 21.69, 17.06. Minor: 166.88, 140.86, 139.40, 138.52, 127.09, 127.08, 126.96, 126.79, $52.00,51.06,43.03,39.19,25.90,21.46,19.87$ (one signal is missing due to overlap). 


\section{Mechanistic Experiments}

\subsection{Control Reactions}

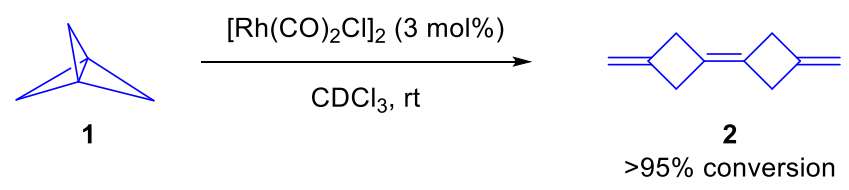

$\left[\mathrm{Rh}(\mathrm{CO})_{2} \mathrm{Cl}\right]_{2}(3 \mathrm{~mol} \%, 4.6 \mathrm{mg})$ and [1.1.1]propellane $(1,0.4 \mathrm{mmol})$ were dissolved in $\mathrm{CDCl}_{3}(0.5$ $\mathrm{mL}$ ) in a J-Young NMR tube under an $\mathrm{N}_{2}$ atmosphere. The mixture was analysed by ${ }^{1} \mathrm{H}$ NMR immediately with 1,3,5-trimethoxybenzene as an internal standard. The ${ }^{1} \mathrm{H}$ NMR showed complete consumption of [1.1.1]propellane and bis(3-methylenecyclobutylidene (2) as the major product.

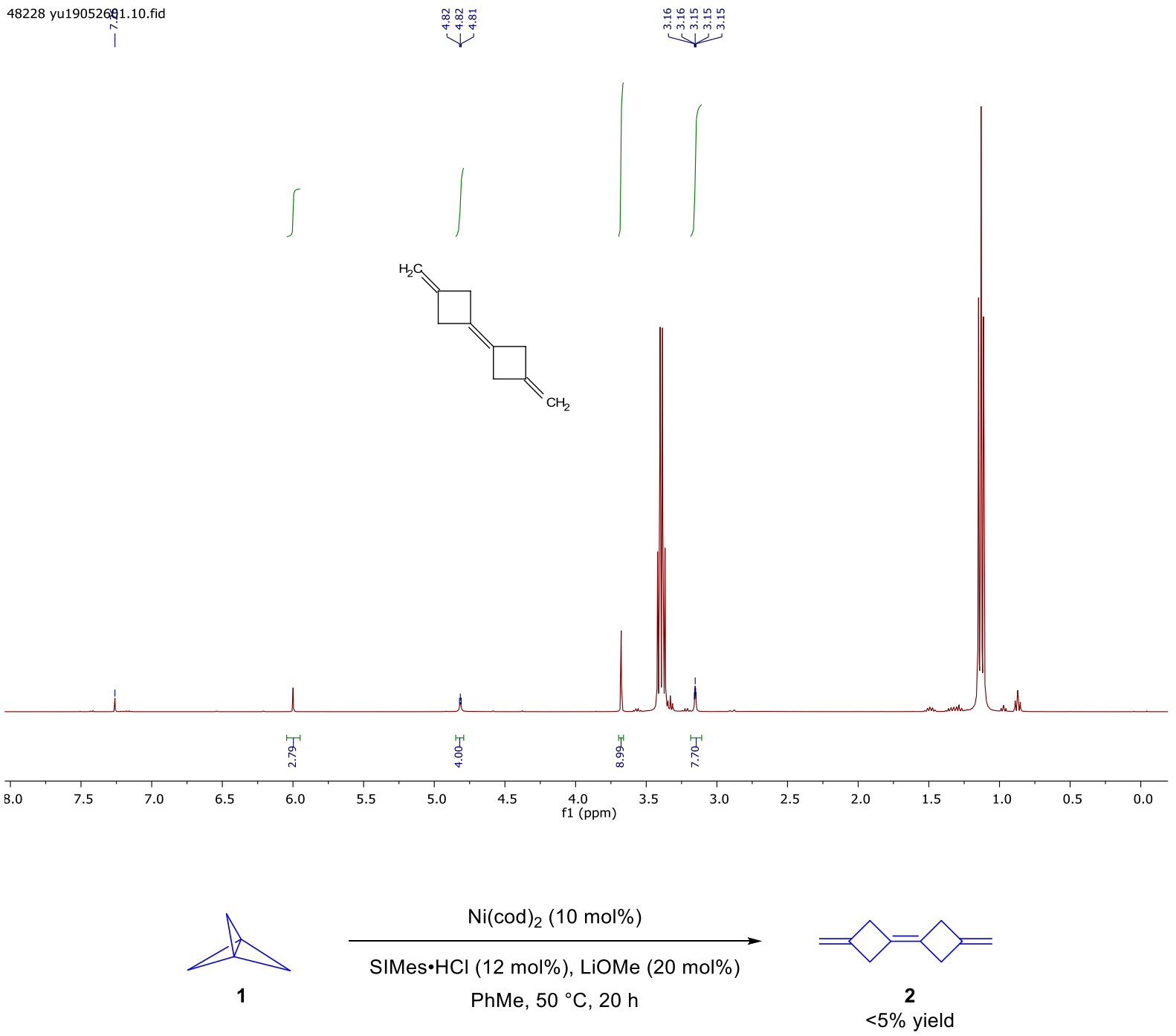

$\mathrm{Ni}(\mathrm{cod})_{2}(10 \mathrm{~mol} \%, 5.6 \mathrm{mg})$, SIMes $\cdot \mathrm{HCl}(12 \mathrm{~mol} \%, 8.3 \mathrm{mg})$ and LiOMe $(20 \mathrm{~mol} \%, 1.5 \mathrm{mg})$ were dissolved in toluene $(2 \mathrm{~mL})$ in a pressure tube under an $\mathrm{N}_{2}$ atmosphere. The mixture was heated to $50{ }^{\circ} \mathrm{C}$ and stirred for $20 \mathrm{~h}$ before allowing to cool to RT. The yield of dimer $\mathbf{2}$ was determined by GC to be $<5 \%$. 


\subsection{Alkene Isomerization Studies}

\section{(E)-4-(Vinyl-2-d $)$-1,1'-biphenyl [(E)-19]}
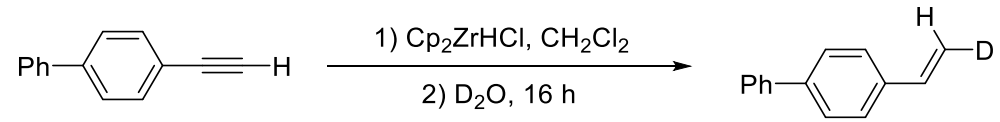

$(E)-19$

4-Ethynylbiphenyl $(890 \mathrm{mg}, 5.0 \mathrm{mmol})$ and $\mathrm{Cp}_{2} \mathrm{ZrHCl}(1.42 \mathrm{~g}, 5.5 \mathrm{mmol})$ were added to a dried flask under an $\mathrm{N}_{2}$ atmosphere. Dry $\mathrm{CH}_{2} \mathrm{Cl}_{2}(12 \mathrm{~mL})$ was added and the resulting mixture was stirred at RT for $45 \mathrm{~min}$ before the addition of $\mathrm{D}_{2} \mathrm{O}(99.9 \%-\mathrm{d}, 1.3 \mathrm{~mL})$. The mixture was stirred at RT for $16 \mathrm{~h}$ before concentrating under reduced pressure. Purification by silica gel flash column chromatography gave $(\boldsymbol{E})-19$ as a white solid in $79 \%$ yield. Analysis of the product by ${ }^{1} \mathrm{H}$ NMR showed $96 \%$ deuterium incorporation.

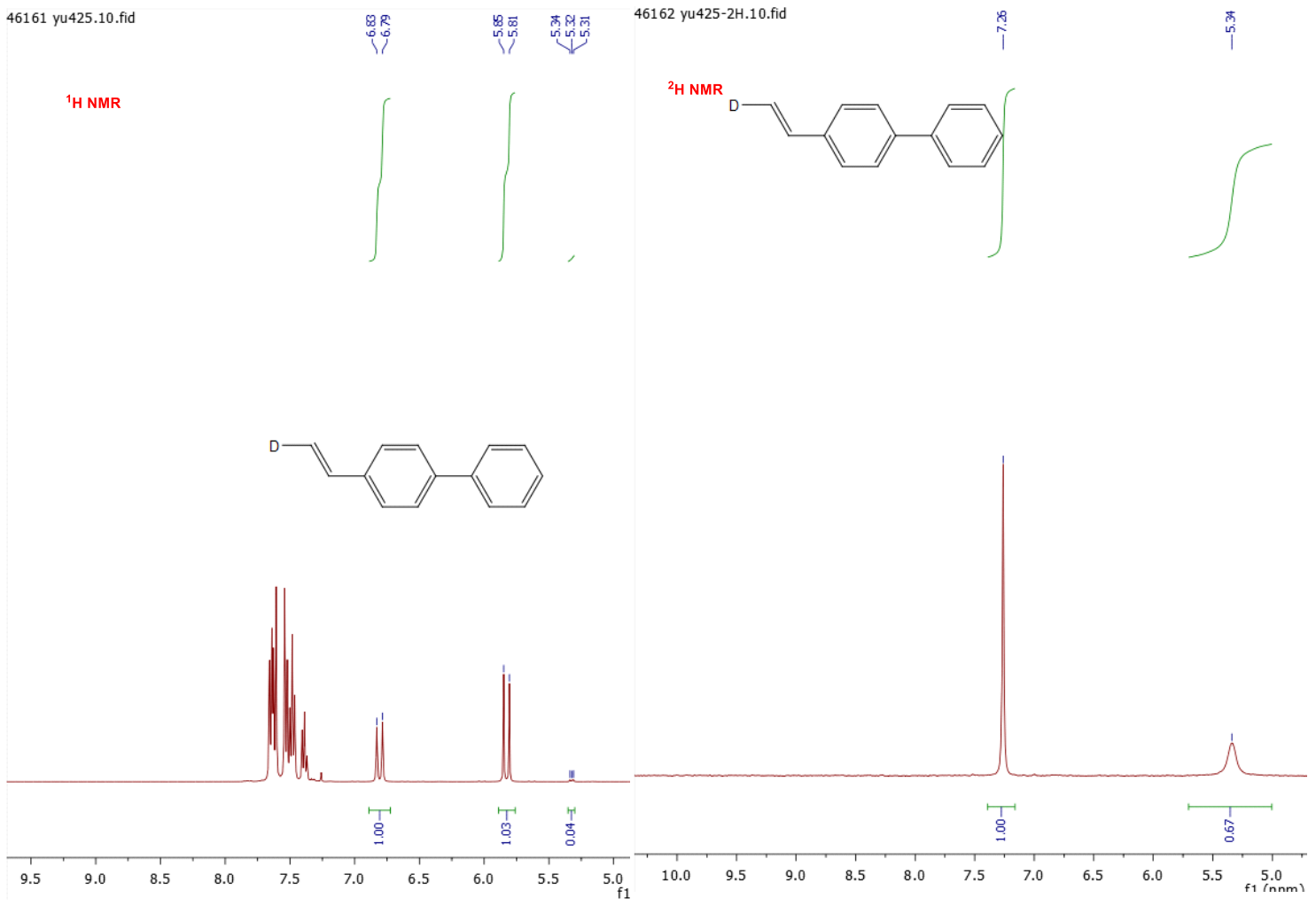


(Z)-4-(Vinyl-2-d $)$-1,1'-biphenyl [(Z)-19]

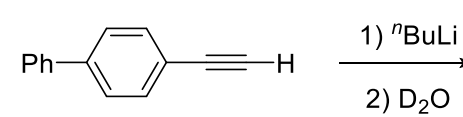<smiles>[2H]C#Cc1ccc(-c2ccccc2)cc1</smiles>

$\frac{\text { 1) } \mathrm{Cp}_{2} \mathrm{ZrHCl}, \mathrm{CH}_{2} \mathrm{Cl}_{2}}{\text { 2) } \mathrm{H}_{2} \mathrm{O}, 16 \mathrm{~h}}$<smiles>[2H]C=Cc1ccc(-c2ccccc2)cc1</smiles>

$(Z)-19$

To an oven-dried Schlenk flask was added 4-ethynylbiphenyl (4 mmol) in THF (5 mL). The solution was cooled to $-78{ }^{\circ} \mathrm{C}$ and ${ }^{n} \mathrm{BuLi}$ (1.6 $\mathrm{M}$ in hexanes, $4.4 \mathrm{mmol}, 1.1$ equiv) was added dropwise. The mixture was stirred at $-78{ }^{\circ} \mathrm{C}$ for $20 \mathrm{~min}$ and then removed from the cold bath and stirred for an additional $20 \mathrm{~min}$. After re-cooling to $-78{ }^{\circ} \mathrm{C}, \mathrm{D}_{2} \mathrm{O}(99.9 \%$-d, $3 \mathrm{~mL})$ was added and the reaction was allowed to warm to rt over $20 \mathrm{~min}$. The mixture was extracted with $\mathrm{Et}_{2} \mathrm{O}$, dried with $\mathrm{Na}_{2} \mathrm{SO}_{4}$, filtered, and concentrated under reduced pressure. Purification by silica gel flash column chromatography gave deuterated 4-ethynylbiphenyl as a white solid. This product was added to a dried flask under an $\mathrm{N}_{2}$ atmosphere, followed by $\mathrm{Cp}_{2} \mathrm{ZrHCl}(4.4 \mathrm{mmol})$ and dry $\mathrm{CH}_{2} \mathrm{Cl}_{2}(12 \mathrm{~mL})$. The resulting mixture was stirred at room temperature for $45 \mathrm{~min}$ before adding $\mathrm{H}_{2} \mathrm{O}(1.3 \mathrm{~mL})$. The mixture was stirred at $\mathrm{RT}$ for $16 \mathrm{~h}$ before concentrating under reduced pressure. Purification by silica gel flash column chromatography gave $(\boldsymbol{Z})-\mathbf{1 9}$ as a white solid in $72 \%$ yield. Analysis of the product by ${ }^{1} \mathrm{H}$ NMR showed $99 \%$ deuterium incorporation.

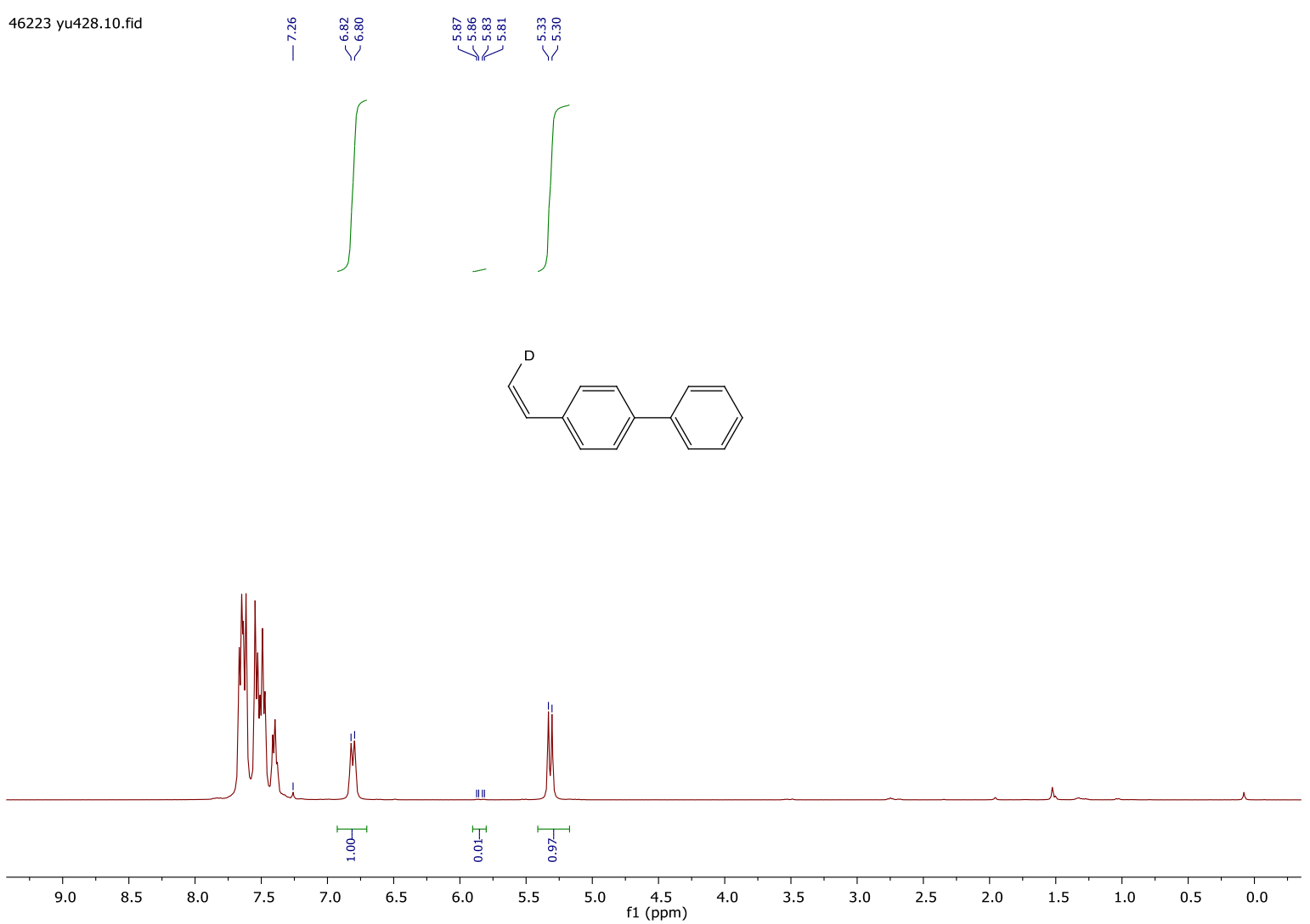




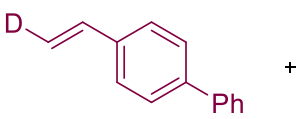

(E)-19

$(96 \%-d)$

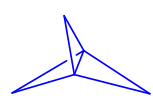

1
$\mathrm{PhMe}, 50{ }^{\circ} \mathrm{C}, 20 \mathrm{~h}$

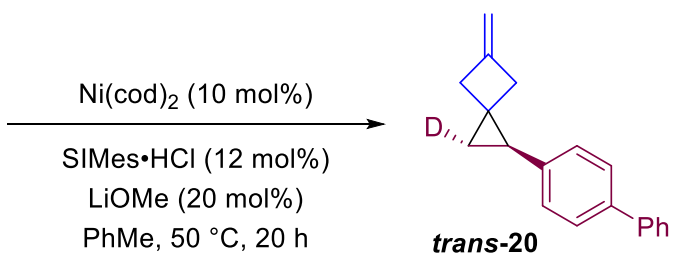

trans: cis $=95: 5$

Following General Procedure A, using Ni( cod) 2 (10 mol\%, $5.6 \mathrm{mg})$, NIMes $\mathrm{HCl}(12 \mathrm{~mol} \%, 8.3 \mathrm{mg})$, biOMe (20 mol\%, $1.5 \mathrm{mg}),(\boldsymbol{E}) \mathbf{- 1 9}(0.20 \mathrm{mmol}, 36 \mathrm{mg})$, and [1.1.1]propellane $(0.80 \mathrm{mmol})$ in toluene ( $3 \mathrm{~mL}$ ). Purification by flash column chromatography with pentane as eluent gave $\mathbf{2 0}$ in $87 \%$ yield as a colourless oil. The trans: cis ratio was determined by ${ }^{1} \mathrm{H}$ NMR and ${ }^{2} \mathrm{H}$ NMR to be 95:5.

46322 yu429.10. fid
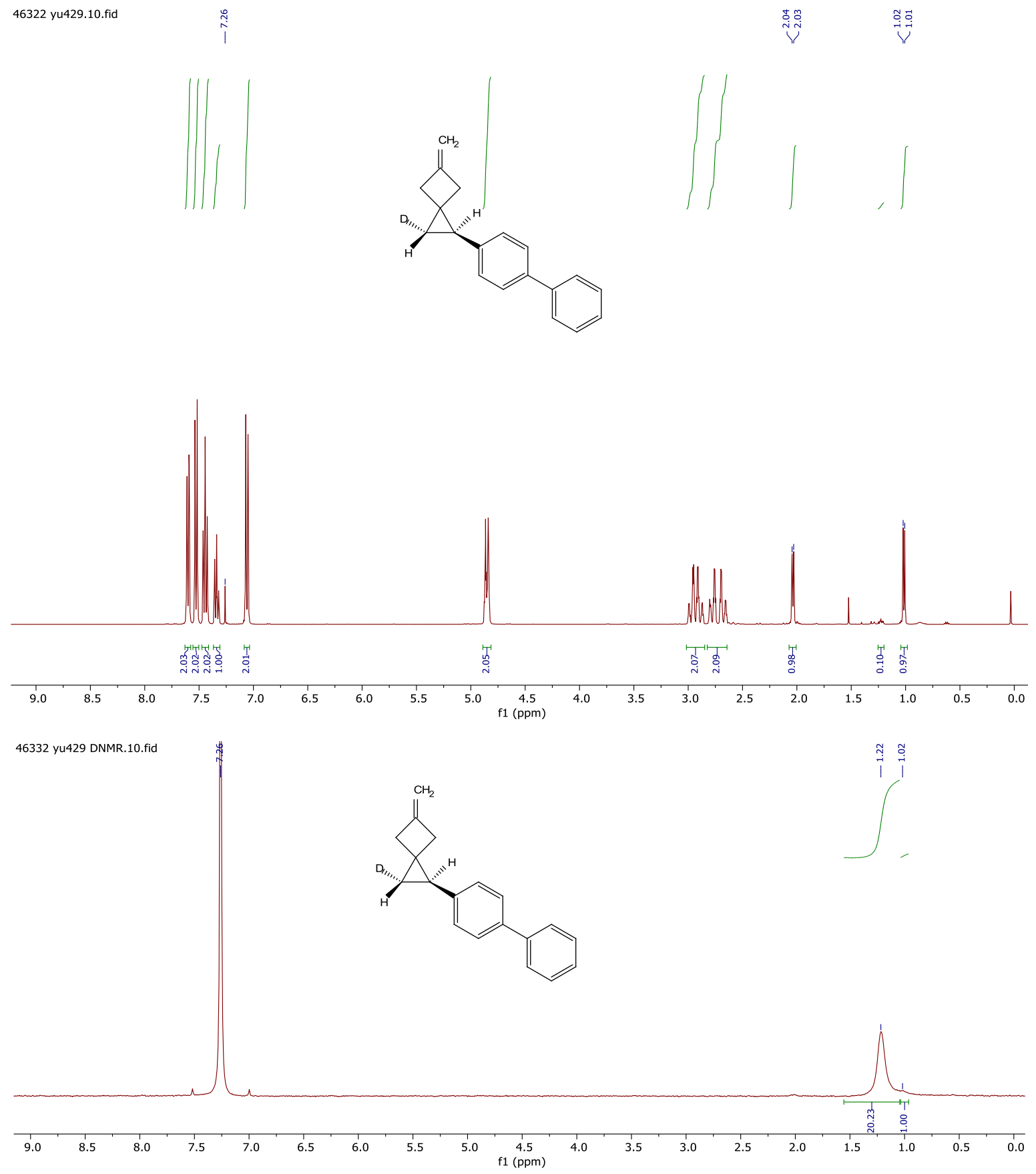

S23 


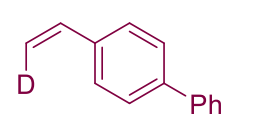

(Z)-19

$(99 \%-d)$

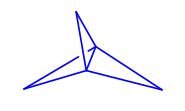

1

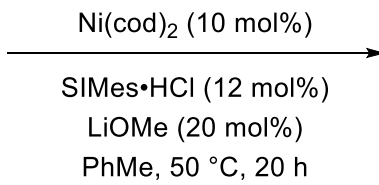

$\mathrm{PhMe}, 50^{\circ} \mathrm{C}, 20 \mathrm{~h}$

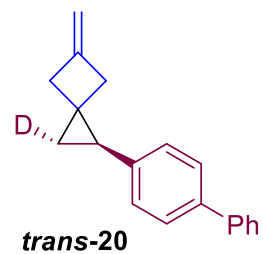

trans: cis $=3: 97$

Following General Procedure A, using Ni(cod) 2 (10 mol\%, $5.6 \mathrm{mg})$, SIMes $\mathrm{HCl}(12 \mathrm{~mol} \%, 8.3 \mathrm{mg})$, LiOMe (20 mol\%, $1.5 \mathrm{mg}),(\boldsymbol{Z})-\mathbf{1 9}(0.20 \mathrm{mmol}, 36 \mathrm{mg})$, and [1.1.1]propellane $(0.80 \mathrm{mmol})$ in toluene ( $3 \mathrm{~mL}$ ). Purification by flash column chromatography with pentane as eluent gave $\mathbf{2 0}$ in $84 \%$ yield as a colourless oil. The trans:cis ratio was determined by ${ }^{1} \mathrm{H}$ NMR and ${ }^{2} \mathrm{H}$ NMR to be 3:97.

46325 yu430.10.fid
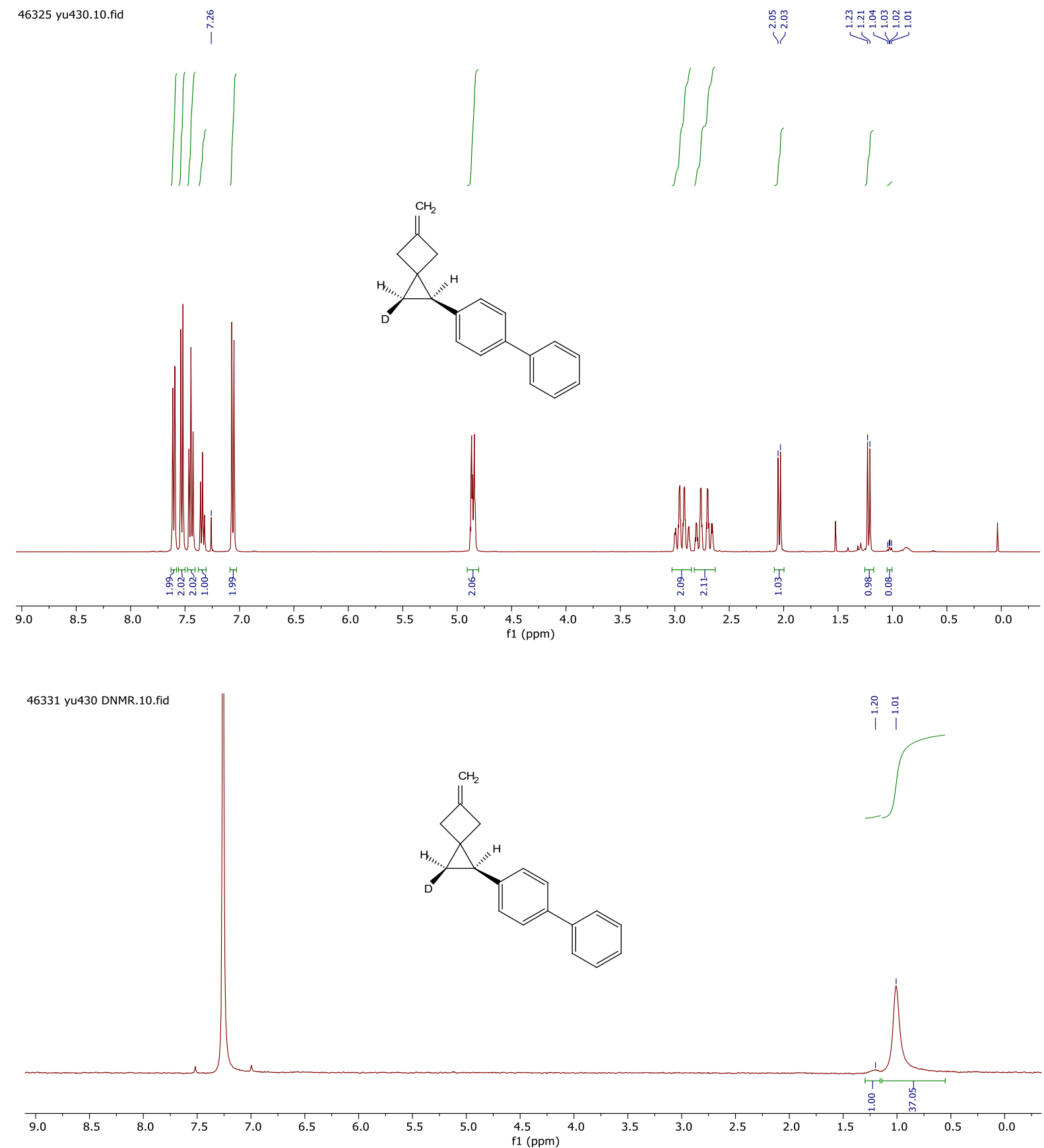
<smiles>[2H]C=Cc1ccc(-c2ccccc2)cc1</smiles>

$(E)-19$ $(96 \%-d)$

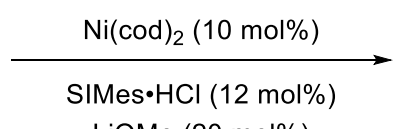

LiOMe $(20 \mathrm{~mol} \%)$

$\mathrm{PhMe}, 50^{\circ} \mathrm{C}, 20 \mathrm{~h}$<smiles>[2H]C=Cc1ccc(-c2ccccc2)cc1</smiles>

(E)-19<smiles>[2H]/C=C\c1ccc(-c2ccccc2)cc1</smiles>

(Z)-19

91\% recovery

$E: Z=50: 50$

$\mathrm{Ni}(\operatorname{cod})_{2}(10 \mathrm{~mol} \%, 5.6 \mathrm{mg})$, SIMes $\cdot \mathrm{HCl}(12 \mathrm{~mol} \%, 8.3 \mathrm{mg})$ and LiOMe $(20 \mathrm{~mol} \%, 1.5 \mathrm{mg})$ were dissolved in toluene $(3 \mathrm{~mL})$ in a pressure tube under an $\mathrm{N}_{2}$ atmosphere. The mixture was stirred for 15 min at $\mathrm{rt}$ before adding $(\boldsymbol{E})-\mathbf{1 9}(0.20 \mathrm{mmol}, 36 \mathrm{mg})$. The reaction mixture was heated to $50{ }^{\circ} \mathrm{C}$ and stirred for $20 \mathrm{~h}$. After allowing to cool to RT, the solvent was removed under reduced pressure and the residue was purified by silica gel flash column chromatography using pentane as eluent to recover starting material in $91 \%$ yield. The $E: Z$ ratio was determined by ${ }^{1} \mathrm{H}$ NMR and ${ }^{2} \mathrm{H}$ NMR to be 50:50.

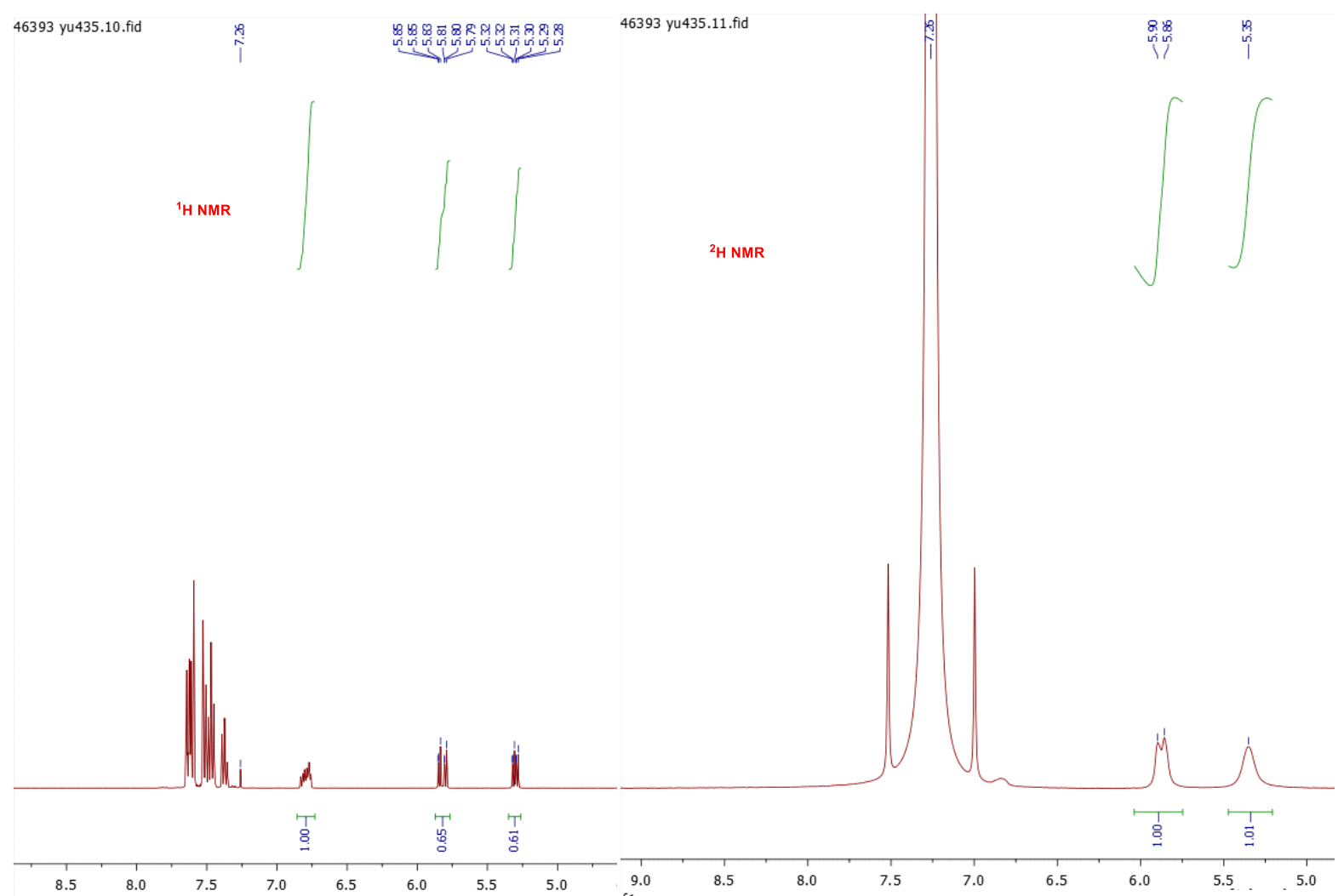




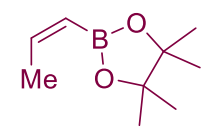

(Z)-11b

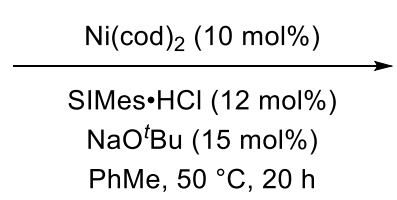

PhMe, $50{ }^{\circ} \mathrm{C}, 20 \mathrm{~h}$<smiles>CC=CB1OC(C)(C)C(C)(C)O1</smiles>

(E)-11b<smiles>C/C=C\B1OC(C)(C)C(C)(C)O1</smiles>

(Z)-11b

$84 \%$ recovery

$E: Z=91: 9$

$\mathrm{Ni}(\operatorname{cod})_{2}(10 \mathrm{~mol} \%, 5.6 \mathrm{mg})$, SIMes $\cdot \mathrm{HCl}(12 \mathrm{~mol} \%, 8.3 \mathrm{mg})$ and $\mathrm{NaO}^{t} \mathrm{Bu}(15 \mathrm{~mol} \%, 2.9 \mathrm{mg})$ were dissolved in toluene $(3 \mathrm{~mL})$ in a pressure tube under an $\mathrm{N}_{2}$ atmosphere. The mixture was stirred for 15 min at $\mathrm{rt}$ before adding $(\boldsymbol{Z})-\mathbf{1 1 b}(0.20 \mathrm{mmol}, 34 \mathrm{mg})$. The reaction mixture was heated to $50{ }^{\circ} \mathrm{C}$ and stirred for $20 \mathrm{~h}$. After allowing to cool to RT, the solvent was removed under reduced pressure and the residue was purified by silica gel flash column chromatography using pentane/diethyl ether (20/1) as eluent to recover starting material in $84 \%$ yield. The $E: Z$ ratio was determined by ${ }^{1} \mathrm{H}$ NMR to be $91: 9$.

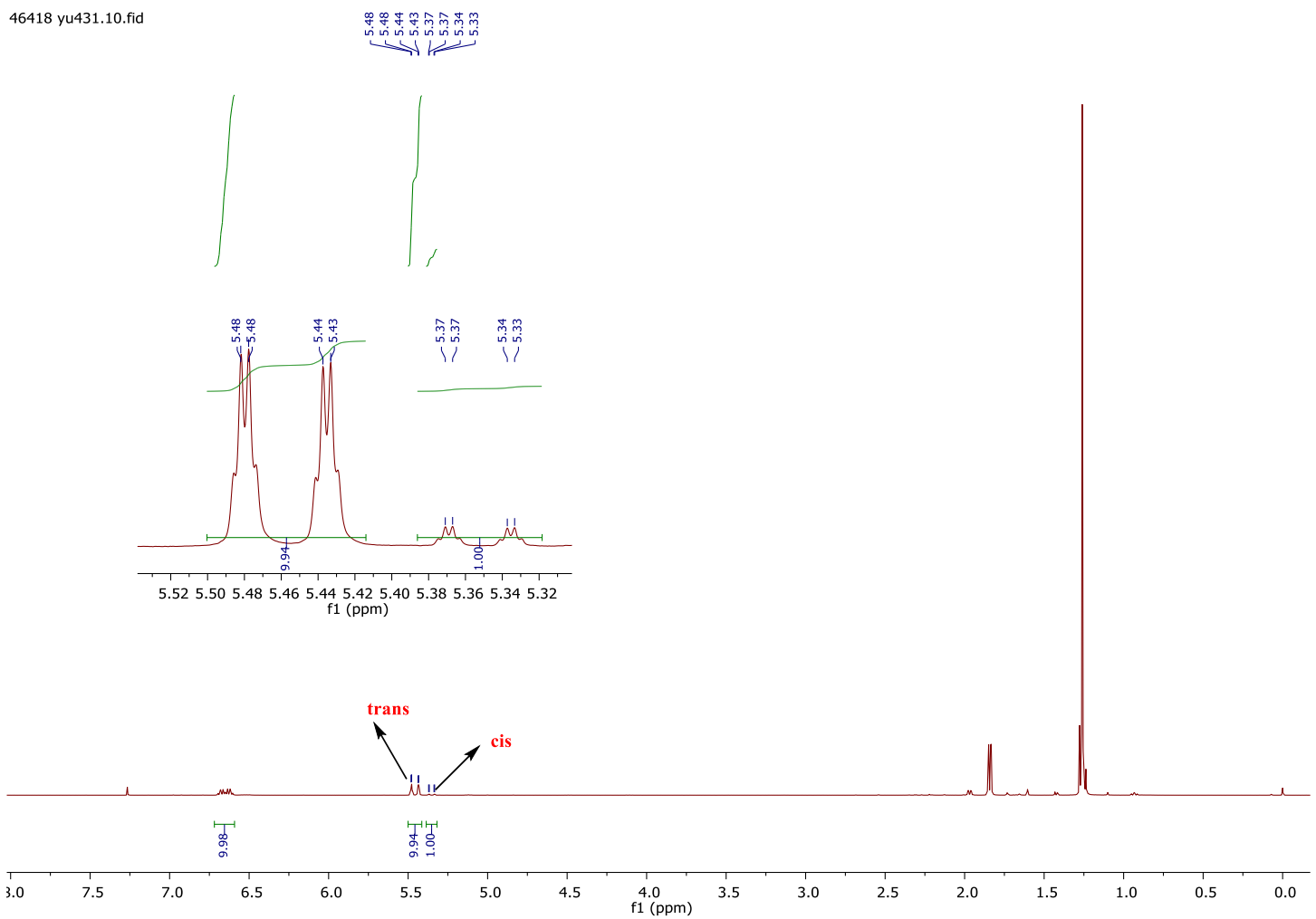




\subsection{Deuteration Studies}

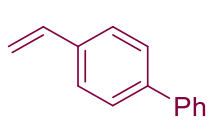

$8 a$

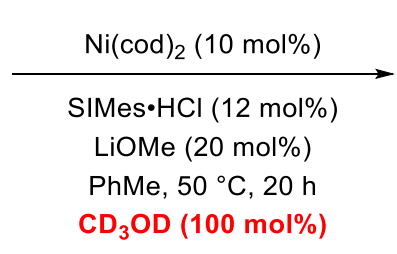

$2.5 \% \mathrm{~d}$

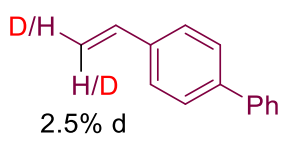

8 a

$94 \%$ recovered

$\mathrm{Ni}(\mathrm{cod})_{2}(10 \mathrm{~mol} \%, 5.6 \mathrm{mg})$, SIMes $\cdot \mathrm{HCl}(12 \mathrm{~mol} \%, 8.3 \mathrm{mg})$ and LiOMe (20 mol\%, $\left.1.5 \mathrm{mg}\right)$ were dissolved in toluene $(1 \mathrm{~mL})$ in a pressure tube under an $\mathrm{N}_{2}$ atmosphere. The mixture was stirred for 15 $\mathrm{min}$ at $\mathrm{rt}$ before adding a solution of $\mathbf{8 a}(0.20 \mathrm{mmol}, 36 \mathrm{mg})$ and $\mathrm{CD}_{3} \mathrm{OD}(0.20 \mathrm{mmol})$ in toluene (2 $\mathrm{mL}$ ). The reaction mixture was heated to $50{ }^{\circ} \mathrm{C}$ and stirred for $20 \mathrm{~h}$. After allowing to cool to RT, the solvent was removed under reduced pressure and the residue was purified by silica gel flash column chromatography using pentane as eluent to recover 8a in $94 \%$ yield $(34 \mathrm{mg})$. The amount of deuterium incorporation was determined by ${ }^{2} \mathrm{H}$ NMR to be $5 \%$ with $\mathrm{CDCl}_{3}(28 \mathrm{mg})$ as an internal standard.

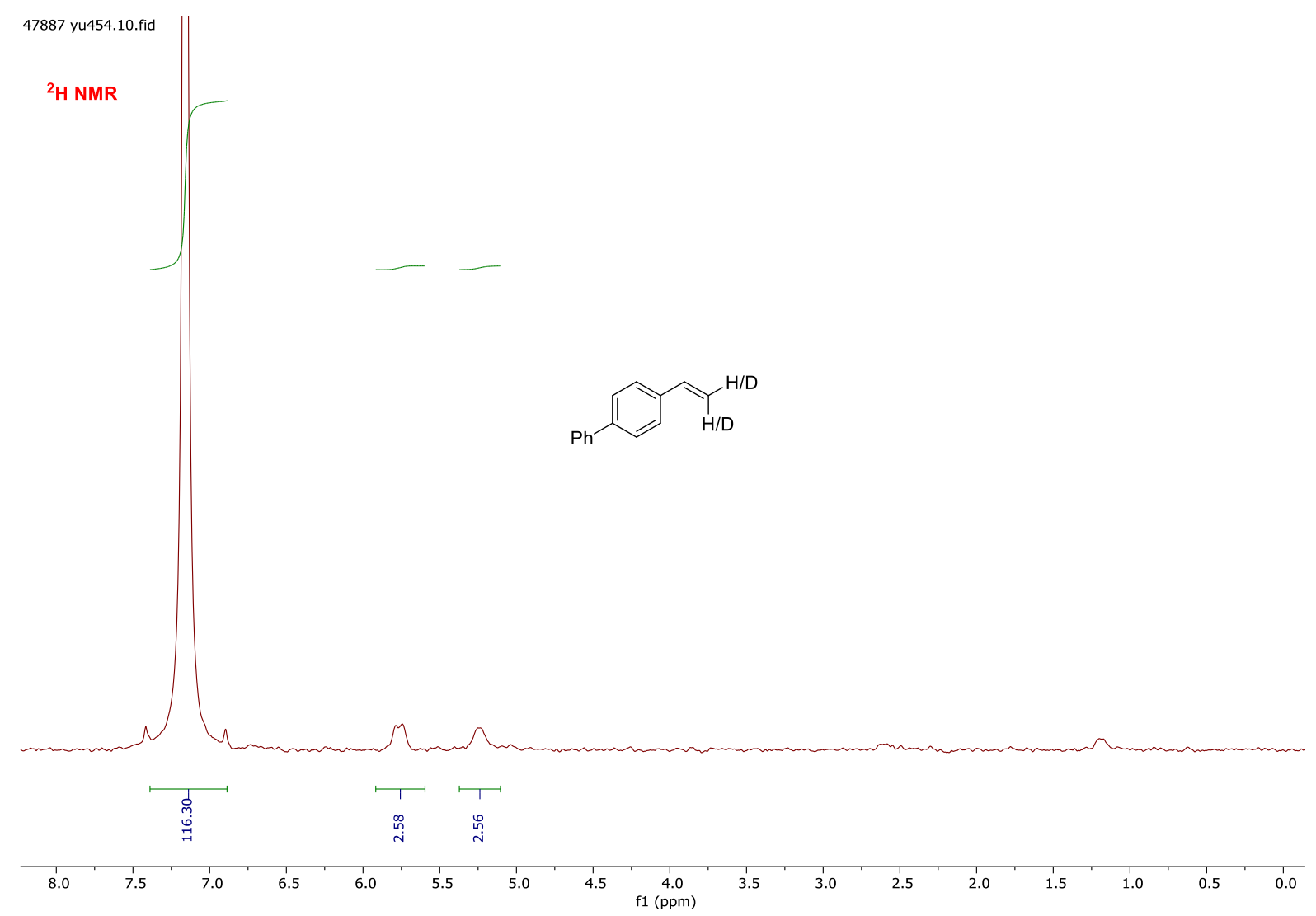




\section{Alternative Substrates}

\subsection{Unreactive Alkenes}

The following alkenes were found to be unreactive under the conditions described in General Procedure A:<smiles>C=CCc1ccccc1</smiles><smiles>C=C(C)c1ccccc1</smiles><smiles>C/C=C/c1ccccc1</smiles><smiles>C=COCCCC</smiles>

\subsection{Terminal Alkynes}

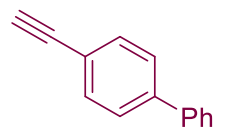

9

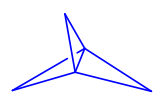

1

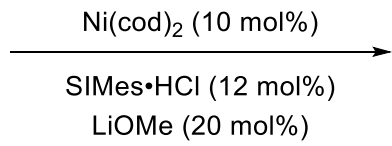

PhMe, rt, 20 h

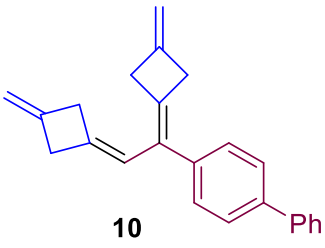

10

The reaction of 4-ethynylbiphenyl was carried out according to General Procedure B, with $\mathrm{Ni}(\operatorname{cod})_{2}$ (10 mol\%, $5.6 \mathrm{mg})$, SIMes $\cdot \mathrm{HCl}$ (12 mol\%, $8.3 \mathrm{mg})$, LiOMe (20 mol\%, $1.5 \mathrm{mg})$, 4-ethynylbiphenyl (9) $(0.20 \mathrm{mmol}, 37 \mathrm{mg})$, and [1.1.1]propellane $(0.80 \mathrm{mmol})$ in toluene $(3 \mathrm{~mL})$. Purification by flash column chromatography with pentane as eluent gave $\mathbf{1 0}(12.4 \mathrm{mg}, 20 \%$ yield) as a colourless oil. HRMS (APCI): Calcd. for $\mathrm{C}_{24} \mathrm{H}_{23}\left([\mathrm{M}+\mathrm{H}]^{+}\right) \mathrm{m} / \mathrm{z} 311.1794$, found $\mathrm{m} / \mathrm{z}$ 311.1786. ${ }^{1} \mathrm{H}$ NMR $(400 \mathrm{MHz}$, Chloroform- $d) \delta 7.68-7.63(\mathrm{~m}, 2 \mathrm{H}), 7.60-7.55(\mathrm{~m}, 2 \mathrm{H}), 7.49-7.44(\mathrm{~m}, 2 \mathrm{H}), 7.39-7.33(\mathrm{~m}, 1 \mathrm{H})$, $7.28-7.24(\mathrm{~m}, 2 \mathrm{H}), 6.07(\mathrm{t}, J=2.5 \mathrm{~Hz}, 1 \mathrm{H}), 4.98(\mathrm{p}, J=2.5 \mathrm{~Hz}, 1 \mathrm{H}), 4.93(\mathrm{p}, J=2.5 \mathrm{~Hz}, 1 \mathrm{H}), 4.81$ (p, $J=2.5 \mathrm{~Hz}, 1 \mathrm{H}), 4.73(\mathrm{p}, J=2.5 \mathrm{~Hz}, 1 \mathrm{H}), 3.54-3.49(\mathrm{~m}, 2 \mathrm{H}), 3.44-3.39(\mathrm{~m}, 2 \mathrm{H}), 3.38-3.33(\mathrm{~m}$, 2H), $2.77-2.68$ (m, 2H). ${ }^{13} \mathrm{C}$ NMR (100 MHz, Chloroform- $d$ ) $\delta 144.9,143.8,140.9,139.1,137.8$, 136.3, 134.5, 131.8, 129.3, 128.7, 127.1, 126.9, 126.5, 119.3, 106.7, 105.9, 41.4, 40.9, 40.0, 39.6. 


\section{Computational Studies}

\section{Computational Methods}

Unless specified, all calculations were performed using Gaussian 09 rev D, ${ }^{5}$ employing the B3LYP functional, ${ }^{6}$ with Grimme's D3 dispersion correction, ${ }^{7}$ without symmetry constraints. Geometry optimizations were performed using the $6-31+\mathrm{G}^{*}$ basis set, ${ }^{8}$ and vibrational frequency calculations were performed for all stationary points to determine whether these were local minima or transition state structures, corresponding to the appearance of no or one imaginary stretching frequency respectively, and to derive the thermochemical corrections for the free energies. The final xyz coordinates and electronic energies of the optimized structures are given below. Solvent effects were modelled by single point calculations performed on the optimized structures using the standard implementation of the SMD method, ${ }^{9}$ with toluene as the solvent. A further single point calculation was performed on each optimized structure using the $6-311++\mathrm{G}^{* *}$ basis set on all atoms. The free energy of each species, $G$ was calculated using Eq. S1 and the results are summarized in Table S1.

$G=G_{\text {small(corr) }}+E_{\text {solv }}+E_{\text {large }}-2 E_{\text {small }}$

$G_{\text {small(corr) }}=$ free energy calculated using $6-31+\mathrm{G}^{*}$ basis set, with standard state correction for solutes ( $1 \mathrm{~atm}$ to $1 \mathrm{M})$

$E_{\text {solv }}=$ solvation energy $\left(6-31+\mathrm{G}^{*}\right)$

$E_{\text {large }}=$ electronic energy calculated using $6-311++\mathrm{G}^{* *}$ basis set

$E_{\text {small }}=$ electronic energy calculated using $6-31+\mathrm{G}^{*}$

Table S1. Calculated electronic and free energies (values in Hartrees), optimized with B3LYP-D3/6$31+\mathrm{G}^{*}$.

\begin{tabular}{llllll}
\hline & $E_{\text {small }}$ & $E_{\text {solv }}$ & $E_{\text {large }}$ & $G_{\text {small(corr })}$ & $G$ \\
\hline RS & -2432.46910 & -2432.49358 & -2432.79164 & -2432.12136 & -2432.46838 \\
styrene & -309.67030 & -309.67946 & -309.73973 & -309.56598 & -309.64456 \\
propellane, 1 & -194.03124 & -194.03496 & -194.06286 & -193.96195 & -193.99729 \\
product, 7b & -503.80246 & -503.78685 & -503.88294 & -503.60865 & -503.67351 \\
$\mathbf{I}^{\mathbf{A}}$ & -2122.74985 & -2122.76811 & -2123.00560 & -2122.52960 & -2122.80361 \\
$\mathbf{I}^{\mathbf{B}}$ & -2316.80172 & -2316.82709 & -2317.09750 & -2316.49299 & -2316.81413 \\
$\mathbf{I}^{\mathbf{B}}$ & -2316.75569 & -2316.78234 & -2317.05211 & -2316.45015 & -2316.77322 \\
$\mathbf{I}^{\mathbf{C}}$ & -2316.82494 & -2316.84655 & -2317.12227 & -2316.51915 & -2316.83809 \\
$\mathbf{I}^{\mathbf{D}}$ & -2316.84207 & -2316.86372 & -2317.13942 & -2316.53167 & -2316.85065 \\
$\mathbf{I}^{\mathbf{E}}$ & -2316.83600 & -2316.85763 & -2317.13262 & -2316.52671 & -2316.84495 \\
$\mathbf{I}^{\mathbf{F}}$ & -2316.80263 & -2316.82649 & -2317.09978 & -2316.49438 & -2316.81539 \\
$\mathbf{I}^{\mathbf{G}}$ & -2316.83504 & -2316.85557 & -2317.13116 & -2316.52104 & -2316.83768 \\
$\mathbf{I}^{\mathbf{H}}$ & -2316.85273 & -2316.87510 & -2317.14907 & -2316.54127 & -2316.85998 \\
TS $^{\mathbf{B C}}$ & -2316.78654 & -2316.81033 & -2317.08308 & -2316.47949 & -2316.79982 \\
TS $^{\mathbf{C D}}$ & -2316.80844 & -2316.83120 & -2317.10560 & -2316.49862 & -2316.81854 \\
TS $^{\mathbf{C D}}$ & -2316.79893 & -2316.82231 & -2317.09603 & -2316.49246 & -2316.81294 \\
TS $^{\mathbf{D E}}$ & -2316.81616 & -2316.83733 & -2317.11313 & -2316.50470 & -2316.82284 \\
TS $^{\mathbf{B F}}$ & -2316.79515 & -2316.81864 & -2317.09060 & -2316.48454 & -2316.80347 \\
TS $^{\mathbf{F G}}$ & -2316.78151 & -2316.80380 & -2317.07880 & -2316.47025 & -2316.78982 \\
TS $^{\mathbf{F G}}$ & -2316.76786 & -2316.79173 & -2317.06487 & -2316.45913 & -2316.78002 \\
TS $^{\mathbf{G H}}$ & -2316.80997 & -2316.83025 & -2317.10612 & -2316.49721 & -2316.81364 \\
\hline
\end{tabular}


Exploring potential radical intermediates. When considering the initial interaction of $\mathbf{I}^{\mathbf{A}}$ with propellane, we also investigated the possibility of single-electron transfer processes. A geometryoptimized triplet diradical ground state analogue $\mathbf{I}^{\mathbf{B} *}$, where one unpaired electron is associated with the now formally $\mathrm{Ni}(\mathrm{I})$ center and the other predominantly with the uncoordinated bridgehead carbon of propellane (Figure S1), was found to be $25.7 \mathrm{kcal} / \mathrm{mol}$ higher in free energy than the closed-shell configuration, while the electronic energy for a vertical transition from the closed-shell singlet species $\mathbf{I}^{\mathbf{B}}$ to the triplet state is $42.4 \mathrm{kcal} / \mathrm{mol}$ (determined by conducting a single point calculation of the triplet configuration, using the optimized geometry of singlet state intermediate $\mathbf{I}^{\mathbf{B}}$; vertical triplet state SCF energy $=-2316.73422211$ hartrees). A broken symmetry (flipspin) calculation was performed on the geometry-optimized triplet state species $\mathbf{I}^{\mathbf{B}}$ using Orca 4.0.1. ${ }^{10}$ This calculation employed the B3LYP functional with D3BJ dispersion correction, ${ }^{11}$ accelerated with the RIJCOSX approximation, ${ }^{12}$ using the $6-31+\mathrm{G}^{*}$ basis set and def2/J auxiliary basis set, ${ }^{13}$ the 'flipspin' routine was applied to the nickel atom (NormalSCF, Grid4, NoFinalGrid) in order to generate a final $\mathrm{S}=0$. An inspection of the Mulliken Population Analysis (see abridged ORCA output file below) showed no significant spin population on the $\mathrm{Ni}$ atom and the propellane ligand after the flipspin calculation, while the UHF corresponding orbitals showed all overlaps to be close to unity, indicating a normal UHF rather than a broken symmetry solution. ${ }^{14}$ The calculated electronic energy difference between the triplet state and the singlet state determined by the flipspin calculation $(18.6 \mathrm{kcal} / \mathrm{mol})$ is almost identical to the electronic energy difference between that calculated for $\mathbf{I}^{\mathbf{B}^{*}}$ (using Gaussian, above) and the vertical singlet calculated at the same level of theory for the triplet geometry $(18.5 \mathrm{kcal} / \mathrm{mol})$. The fact that the broken symmetry calculation on $\mathbf{I}^{\mathbf{B}^{*}}$ did not reveal a singlet diradical as opposed to a closed-shell configuration as the preferred singlet state is perhaps unsurprising in view of the character of the triplet species' SOMOs (Figure S1). Accordingly, single-electron transfer pathways were not considered further, especially considering that it has previously been shown that the barrier to ring-opening of the bicyclo[1.1.1]pentyl radical is at least $26 \mathrm{kcal} / \mathrm{mol} .{ }^{15}$

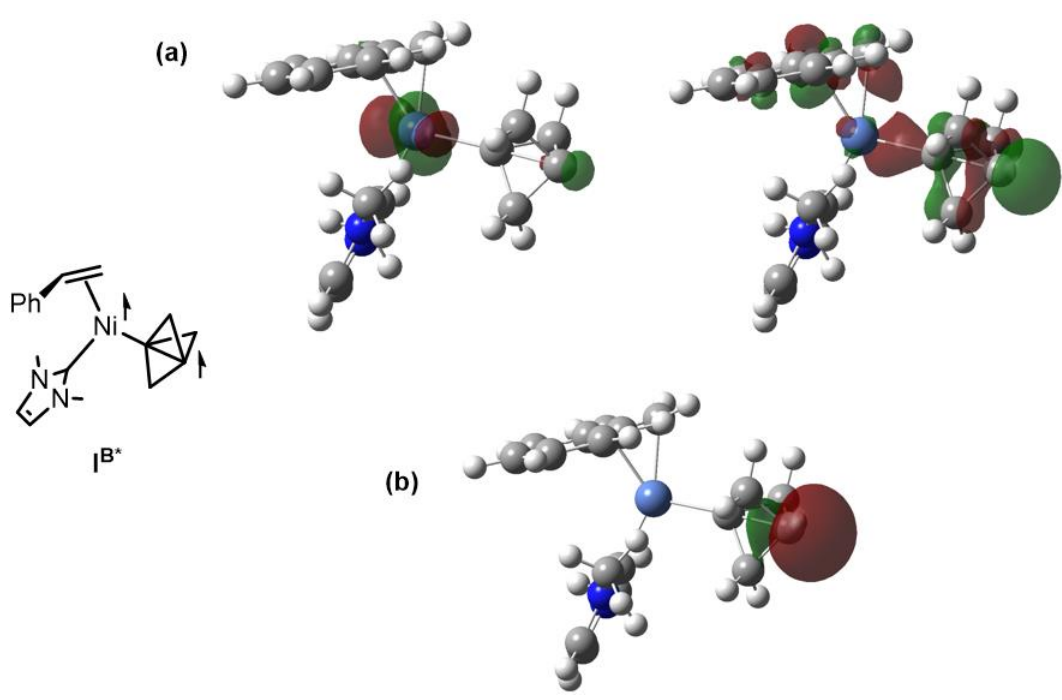

Figure S1. (a) Ni and propellane-based SOMOs and (b) singly occupied natural bond order (NBO) on propellane for the triplet diradical $\mathbf{I}^{\mathbf{*}}\left(\right.$ isovalue $\left.=0.05\left(\text { electron } / \mathrm{bohr}^{3}\right)^{1 / 2}\right)$. 
Alternative reaction pathways. An alternative pathway was considered in which $\mathbf{I}^{\mathbf{F}}$ is transformed into metallacyclobutane $\mathbf{I}^{\mathbf{G}}$ via reaction of the coordinated styrene, which is followed by reductive elimination to give $\mathbf{I}^{\mathbf{H}}$ (Scheme S1). However, the transition state $\mathbf{T S}^{\mathbf{F G}}$ is over $6 \mathrm{kcal} / \mathrm{mol}$ higher in free energy than $\mathbf{T S}^{\mathbf{B C}}$, indicating this is not likely to occur, at least in the model reaction. However, this difference in energy is sufficiently small that changing the ligand or alkene substrate may make this alternative pathway competitive. Again, the regioisomeric transition state $\mathbf{T S}^{\mathbf{F G}}$, in which $\mathrm{C}-\mathrm{C}$ bond-formation occurs at the benzylic carbon, was considered but is $6.1 \mathrm{kcal} / \mathrm{mol}$ higher in free energy than $\mathbf{T S}^{\mathrm{FG}}$.
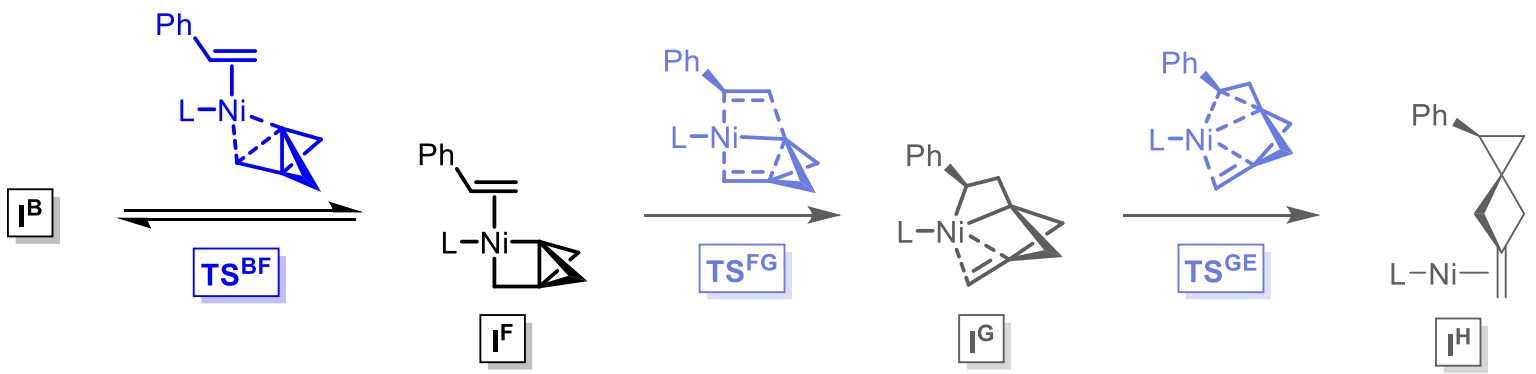

$\mathbf{I}^{\mathrm{G}}$
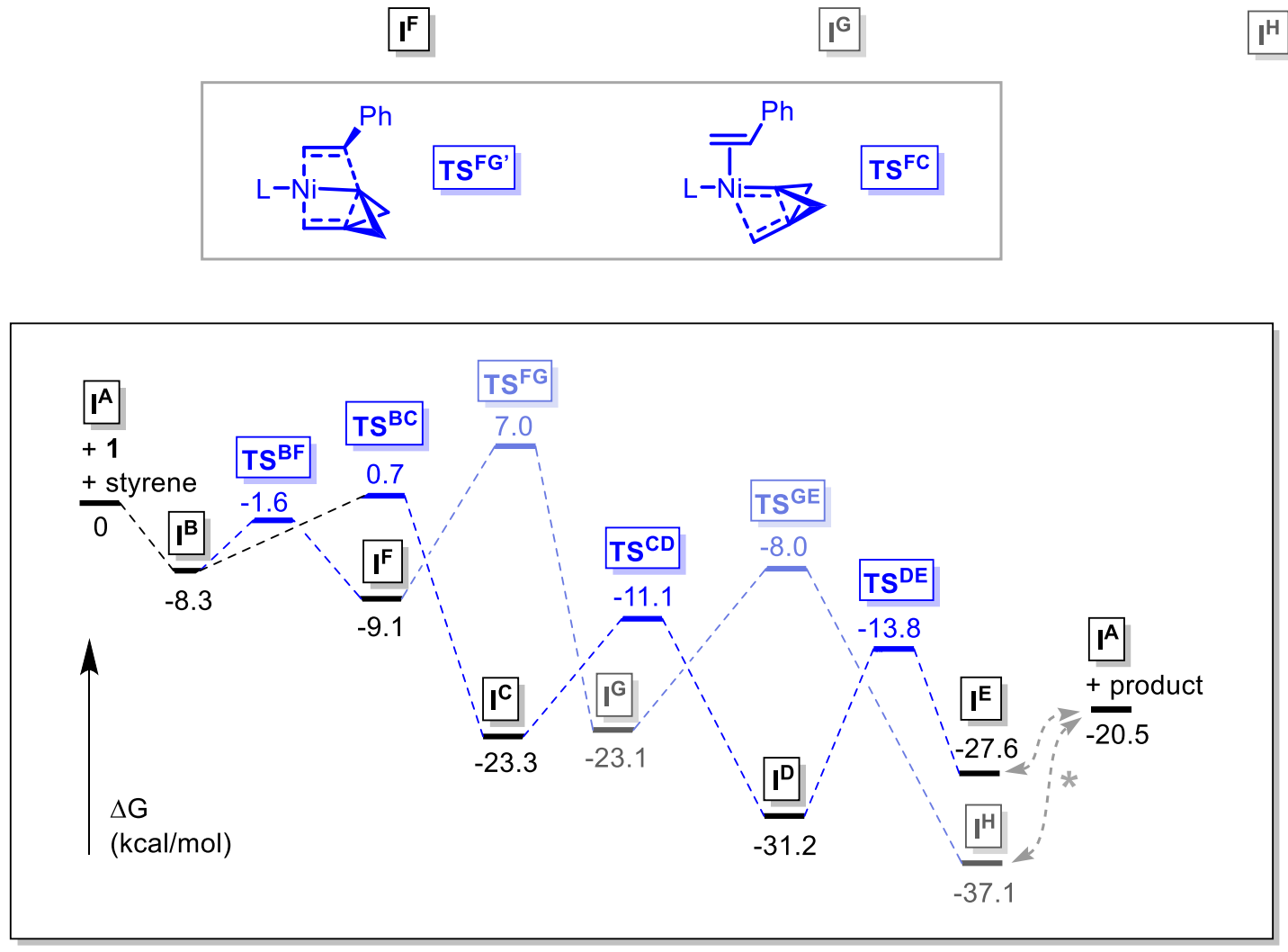

Scheme S1. An alternative pathway for the conversion of $\mathbf{I}^{\mathbf{F}}$ to $\mathbf{I}^{\mathbf{G}}$ and a comparison of the energetics versus those for the main cycle. *The individual ligand substitution steps ( $\mathbf{I}^{\mathbf{E}}$ to $\mathbf{I}^{\mathbf{A}}$ and $\mathbf{I}^{\mathbf{H}}$ to $\mathbf{I}^{\mathbf{A}}$ ) were not modelled. 
Another mechanism may be envisaged in which the nickelacycle $\mathbf{I}^{\mathbf{F}}$ is transformed into the carbene complex $\mathbf{I}^{\mathbf{C}}$ via the ring-opening transition state $\mathbf{T S}^{\mathbf{F C}}$ (Scheme S1). In an attempt to locate this putative transition state, a relaxed scan of the potential energy surface was undertaken with incremental increases in the $\mathrm{Ni}-\mathrm{CH}_{2}$ separation employing loose optimization criteria, and the results are summarized in Figure S2. As can be seen, rather than undergoing ring-opening to form the desired intermediate $\mathbf{I}^{\mathbf{C}}, \mathbf{I}^{\mathbf{B}}$ is reformed by reductive elimination instead.

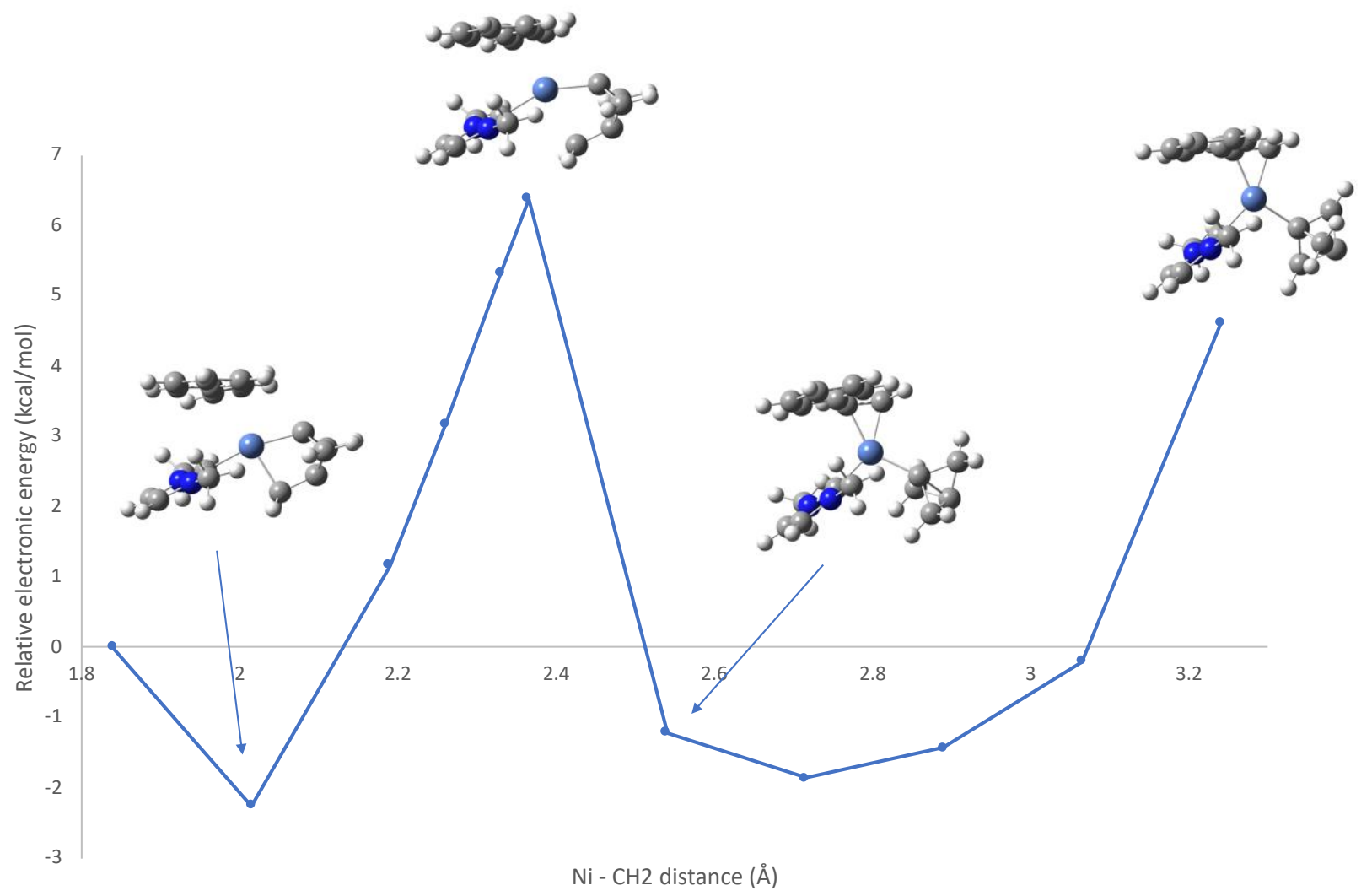

Figure S2. Results of a relaxed PES scan of increasing $\mathrm{Ni}-\mathrm{CH}_{2}$ distance for intermediate $\mathbf{I}^{\mathbf{F}}$ showing reductive elimination to intermediate $\mathbf{I}^{\mathbf{B}}$ rather than formation of carbene intermediate $\mathbf{I}^{\mathbf{C}}$. 
AIM studies. In order to explore the AIM topological properties of $\mathbf{I}^{\mathbf{B}}, \mathbf{I}^{\mathbf{C}}$, [1.1.1]-propellane (1) and [2.1.1]-propellane their geometries were first optimized (B3LYP/6-311++G**), without symmetry constraints (the first three starting from the geometries obtained above with smaller basis set; final xyz coordinates and electronic energies of the optimized structures are given below) then the properties were obtained from the wave functions using the AIMALL suite of programs. ${ }^{16}$ Molecular graphs for the four species are shown in Figures S3 - S6.

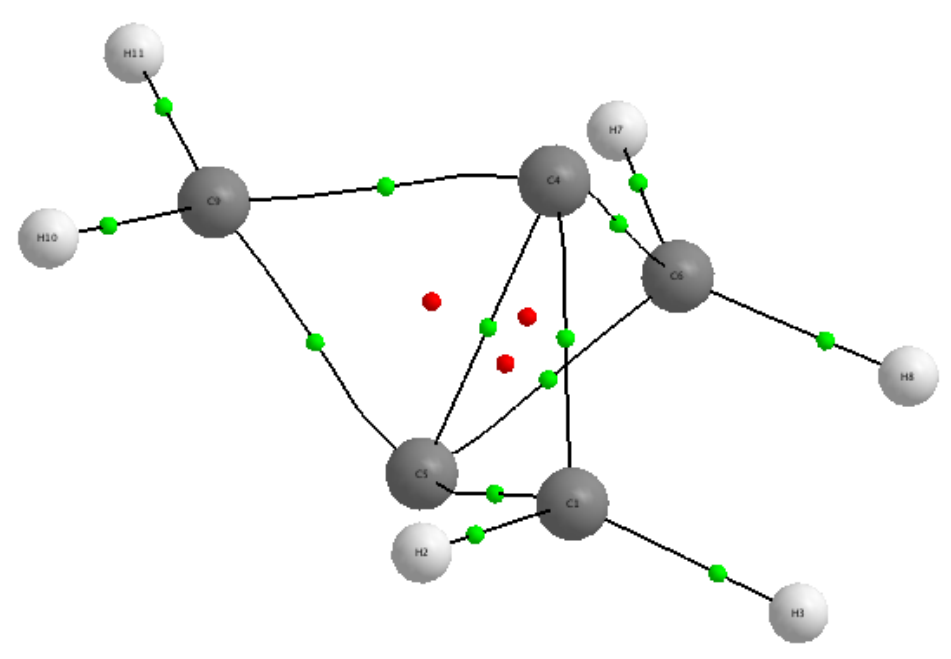

Figure S3. Molecular graph of propellane, 1, bond critical points (bcp) shown as small green spheres, ring critical points ( $\mathrm{rcp}$ ) as small red spheres.

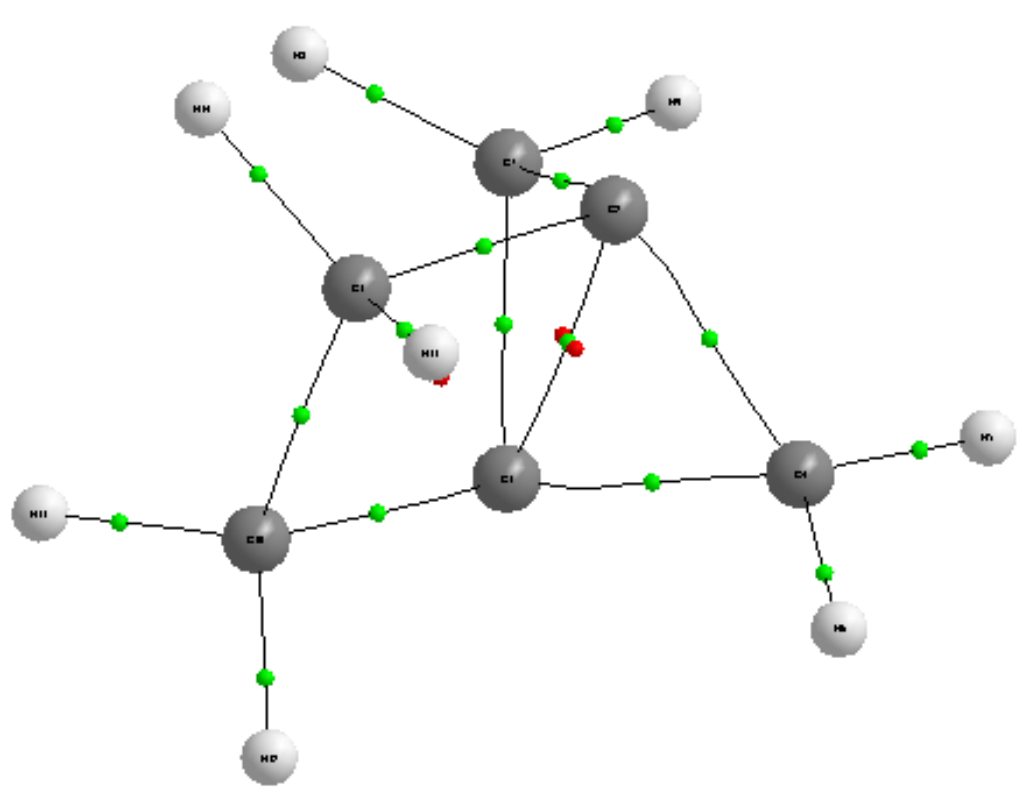

Figure S4. Molecular graph of [2.1.1]-propellane, bond critical points (bcp) shown as small green spheres, ring critical points (rcp) as small red spheres. 


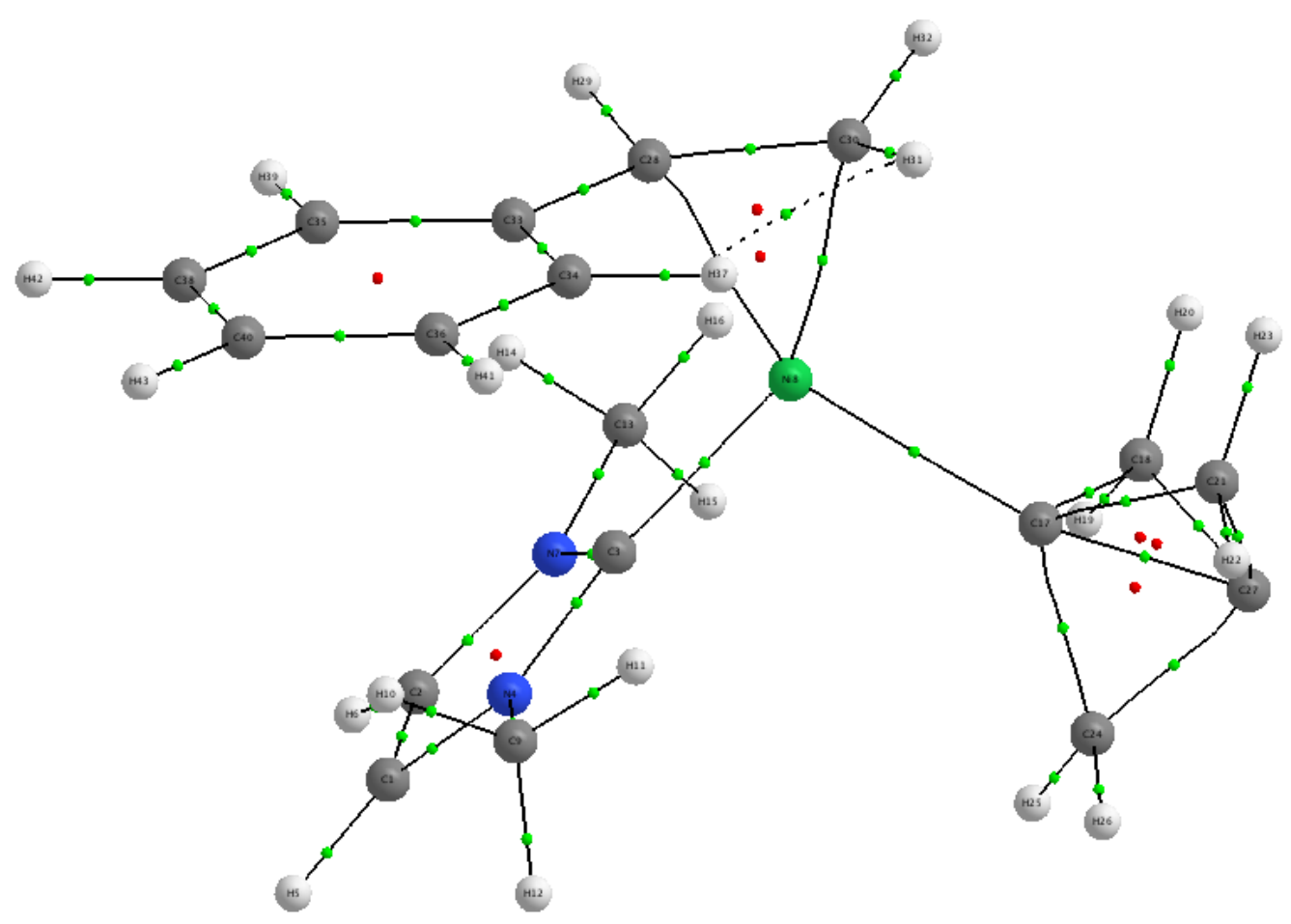

Figure S5. Molecular graph of intermediate $\mathbf{I}^{\mathbf{B}}$, bond critical points (bcp) shown as small green spheres, ring critical points ( $\mathrm{rcp}$ ) as small red spheres.

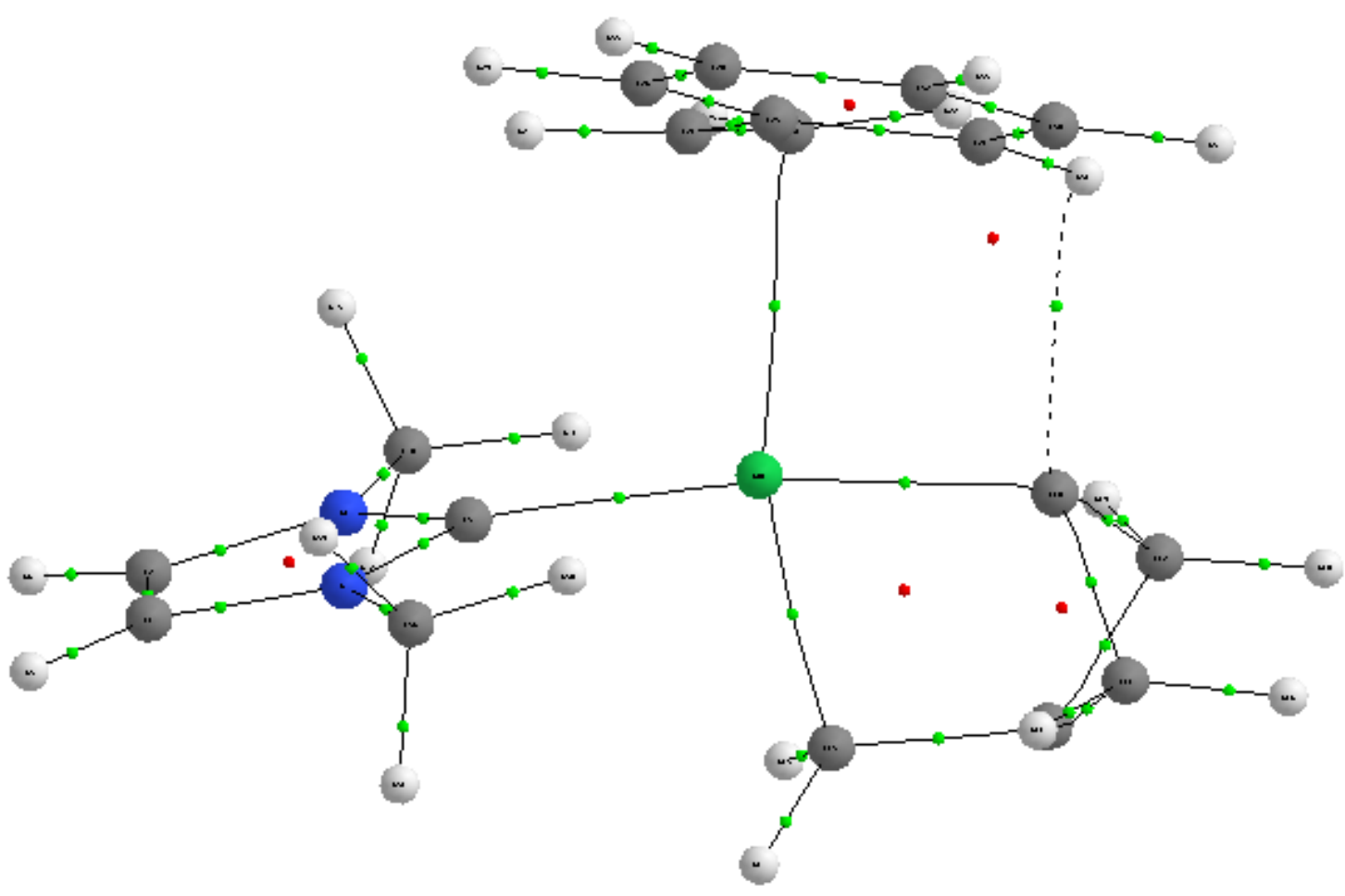

Figure S6. Molecular graph of intermediate $\mathbf{I}^{\mathbf{F}}$, bond critical points (bcp) shown as small green spheres, ring critical points (rcp) as small red spheres. 


\section{Optimized Cartesian Coordinates}

(a) (B3LYP-D3/6-31+G*)

\section{RS}

SCF Energy: -2432.46910067 hartrees

\begin{tabular}{|c|c|c|c|}
\hline C & 0.59777 & -0.08315 & 2.28923 \\
\hline $\mathrm{C}$ & -0.64497 & 0.24509 & 2.72647 \\
\hline $\mathrm{C}$ & -0.78637 & -0.09969 & 0.47522 \\
\hline $\mathrm{N}$ & 0.49374 & -0.28797 & 0.91794 \\
\hline $\mathrm{H}$ & 1.53085 & -0.18995 & 2.82220 \\
\hline $\mathrm{H}$ & -1.00788 & 0.48486 & 3.71488 \\
\hline $\mathrm{N}$ & -1.47444 & 0.22807 & 1.61081 \\
\hline $\mathrm{C}$ & -2.25441 & -1.77550 & -2.39950 \\
\hline $\mathrm{H}$ & -3.20089 & -1.44716 & -2.82759 \\
\hline $\mathrm{C}$ & -2.21692 & -2.08490 & -1.02721 \\
\hline $\mathrm{H}$ & -1.48666 & -2.78517 & -0.62332 \\
\hline $\mathrm{H}$ & -3.13851 & -2.02471 & -0.44814 \\
\hline $\mathrm{C}$ & -1.25182 & -2.22193 & -3.38667 \\
\hline $\mathrm{C}$ & 0.01027 & -2.73275 & -3.01754 \\
\hline $\mathrm{C}$ & -1.52998 & -2.11313 & -4.76324 \\
\hline $\mathrm{C}$ & 0.94724 & -3.11006 & -3.97889 \\
\hline $\mathrm{H}$ & 0.25933 & -2.82565 & -1.96429 \\
\hline $\mathrm{C}$ & -0.59247 & -2.48798 & -5.72737 \\
\hline $\mathrm{H}$ & -2.49979 & -1.73161 & -5.07424 \\
\hline $\mathrm{C}$ & 0.65545 & -2.98761 & -5.34268 \\
\hline $\mathrm{H}$ & 1.91220 & -3.50135 & -3.66328 \\
\hline $\mathrm{H}$ & -0.83902 & -2.39229 & -6.78249 \\
\hline $\mathrm{H}$ & 1.38722 & -3.28041 & -6.09119 \\
\hline $\mathrm{C}$ & -2.89741 & 0.53488 & 1.63578 \\
\hline $\mathrm{H}$ & -3.05941 & 1.58680 & 1.89502 \\
\hline $\mathrm{H}$ & -3.30093 & 0.34399 & 0.63980 \\
\hline $\mathrm{H}$ & -3.40901 & -0.10198 & 2.36474 \\
\hline $\mathrm{C}$ & 1.60456 & -0.66080 & 0.05363 \\
\hline $\mathrm{H}$ & 2.00392 & -1.63855 & 0.34390 \\
\hline $\mathrm{H}$ & 1.23248 & -0.71350 & -0.97123 \\
\hline $\mathrm{H}$ & 2.40034 & 0.08902 & 0.11329 \\
\hline $\mathrm{C}$ & -1.65749 & 1.04341 & -2.86227 \\
\hline $\mathrm{H}$ & -1.20780 & 0.60257 & -3.75123 \\
\hline $\mathrm{C}$ & -0.80305 & 1.55360 & -1.86777 \\
\hline $\mathrm{H}$ & -1.12739 & 2.34529 & -1.19341 \\
\hline $\mathrm{H}$ & 0.27398 & 1.52921 & -2.03385 \\
\hline $\mathrm{C}$ & -3.08388 & 1.39667 & -3.00262 \\
\hline $\mathrm{C}$ & -3.76741 & 1.08088 & -4.19304 \\
\hline $\mathrm{C}$ & -3.82276 & 2.01848 & -1.97437 \\
\hline $\mathrm{C}$ & -5.12515 & 1.36506 & -4.35025 \\
\hline $\mathrm{H}$ & -3.21779 & 0.60977 & -5.00474 \\
\hline $\mathrm{C}$ & -5.17838 & 2.30553 & -2.13031 \\
\hline $\mathrm{H}$ & -3.32742 & 2.26984 & -1.04084 \\
\hline $\mathrm{C}$ & -5.84243 & 1.97853 & -3.31874 \\
\hline $\mathrm{H}$ & -5.62321 & 1.10936 & -5.28286 \\
\hline $\mathrm{H}$ & -5.72180 & 2.78693 & -1.31989 \\
\hline $\mathrm{H}$ & -6.89942 & 2.20178 & -3.43869 \\
\hline $\mathrm{Ni}$ & -1.45359 & -0.25384 & -1.2991( \\
\hline
\end{tabular}




\begin{tabular}{lrrr}
\multicolumn{4}{l}{ Styrene } \\
SCF Energy: -309.670302977 & hartrees \\
$\mathrm{C}$ & -1.36863 & 0.07790 & 0.01805 \\
$\mathrm{C}$ & 0.02683 & 0.13201 & 0.01877 \\
$\mathrm{C}$ & 0.71018 & 1.36187 & 0.01847 \\
$\mathrm{C}$ & -0.05736 & 2.54305 & 0.01746 \\
$\mathrm{C}$ & -1.44972 & 2.49197 & 0.01675 \\
$\mathrm{C}$ & -2.11419 & 1.25898 & 0.01704 \\
$\mathrm{H}$ & -1.87144 & -0.88588 & 0.01829 \\
$\mathrm{H}$ & 0.59978 & -0.79307 & 0.01956 \\
$\mathrm{H}$ & 0.43684 & 3.51042 & 0.01724 \\
$\mathrm{H}$ & -2.02124 & 3.41680 & 0.01598 \\
$\mathrm{H}$ & -3.20041 & 1.22251 & 0.01650 \\
$\mathrm{C}$ & 2.18370 & 1.35742 & 0.01924 \\
$\mathrm{H}$ & 2.63305 & 0.36369 & 0.02034 \\
$\mathrm{C}$ & 3.00604 & 2.41789 & 0.01872 \\
$\mathrm{H}$ & 2.64678 & 3.44387 & 0.01760 \\
$\mathrm{H}$ & 4.08395 & 2.28564 & 0.01940
\end{tabular}

Propellane, 1

SCF Energy: -194.031242626 hartrees

$\begin{array}{lrrc}\mathrm{C} & 1.69232 & 1.00011 & 1.86086 \\ \mathrm{H} & 1.87078 & 0.07729 & 2.41942 \\ \mathrm{H} & 1.87064 & 1.92307 & 2.41924 \\ \mathrm{C} & 0.54165 & 0.99993 & 0.86476 \\ \mathrm{C} & 2.05149 & 1.00000 & 0.38188 \\ \mathrm{C} & 1.09865 & 2.12525 & 0.00422 \\ \mathrm{H} & 0.76600 & 2.17138 & -1.03628 \\ \mathrm{H} & 1.25269 & 3.09454 & 0.48612 \\ \mathrm{C} & 1.09883 & -0.12547 & 0.00443 \\ \mathrm{H} & 1.25302 & -1.09464 & 0.48651 \\ \mathrm{H} & 0.76619 & -0.17185 & -1.03606\end{array}$

Product, $\mathbf{7 b}$

SCF Energy: -503.802462138 hartrees

$\begin{array}{lrrr}\mathrm{H} & -0.14909 & 0.59998 & -1.42381 \\ \mathrm{C} & -0.22148 & 0.89146 & -0.36868 \\ \mathrm{C} & 0.56912 & -0.01525 & 0.59455 \\ \mathrm{C} & -1.49467 & 0.42351 & 0.33166 \\ \mathrm{C} & -0.72925 & -0.53240 & 1.24335 \\ \mathrm{H} & -0.93529 & -1.59575 & 1.06386 \\ \mathrm{H} & -0.82915 & -0.33272 & 2.31785 \\ \mathrm{H} & -0.01245 & 1.96562 & -0.28979 \\ \mathrm{C} & 1.79978 & -0.83840 & 0.26617 \\ \mathrm{H} & 1.79725 & -1.84154 & 0.69008 \\ \mathrm{C} & 1.85806 & 0.29137 & 1.28244 \\ \mathrm{H} & 1.96365 & 0.01509 & 2.32959 \\ \mathrm{H} & 2.39127 & 1.19752 & 1.00287 \\ \mathrm{C} & -2.77883 & 0.75123 & 0.21557 \\ \mathrm{H} & -3.11361 & 1.49006 & -0.50958 \\ \mathrm{H} & -3.54199 & 0.29254 & 0.84081 \\ \mathrm{C} & 2.45450 & -0.75829 & -1.06686 \\ \mathrm{C} & 3.65178 & -0.69605 & -3.62537 \\ \mathrm{C} & 2.73456 & 0.46182 & -1.70362\end{array}$




$\begin{array}{lrrr}\mathrm{C} & 2.78673 & -1.94430 & -1.74046 \\ \mathrm{C} & 3.37992 & -1.91706 & -3.00375 \\ \mathrm{C} & 3.32592 & 0.49290 & -2.96747 \\ \mathrm{H} & 2.49243 & 1.39936 & -1.21163 \\ \mathrm{H} & 2.57806 & -2.89984 & -1.26444 \\ \mathrm{H} & 3.62993 & -2.85060 & -3.50127 \\ \mathrm{H} & 3.53353 & 1.45034 & -3.43850 \\ \mathrm{H} & 4.11313 & -0.67053 & -4.60877\end{array}$

$\mathbf{I}^{\mathbf{A}}$

SCF Energy: -2122.7498492 hartrees

$\begin{array}{llll}\mathrm{C} & 2.85535 & 2.24888 & 0.24217 \\ \mathrm{C} & 1.68697 & 2.93225 & 0.37832 \\ \mathrm{C} & 1.18450 & 0.91580 & -0.56388 \\ \mathrm{~N} & 2.53130 & 1.02850 & -0.32802 \\ \mathrm{H} & 3.86907 & 2.52314 & 0.49394 \\ \mathrm{H} & 1.48596 & 3.91368 & 0.78169 \\ \mathrm{~N} & 0.68749 & 2.11583 & -0.12368 \\ \mathrm{Ni} & 0.21758 & -0.56293 & -1.30155 \\ \mathrm{C} & -0.77305 & -2.05129 & -2.13098 \\ \mathrm{H} & -1.16511 & -1.76799 & -3.11049 \\ \mathrm{C} & -1.39174 & -1.62319 & -0.93692 \\ \mathrm{H} & -1.21929 & -2.21183 & -0.03124 \\ \mathrm{C} & -2.50715 & -0.66396 & -0.81563 \\ \mathrm{C} & -3.08142 & -0.00105 & -1.92084 \\ \mathrm{C} & -3.00781 & -0.34915 & 0.46438 \\ \mathrm{C} & -4.09006 & 0.94609 & -1.74837 \\ \mathrm{H} & -2.71951 & -0.22112 & -2.92057 \\ \mathrm{C} & -4.01702 & 0.59867 & 0.63872 \\ \mathrm{H} & -2.58027 & -0.84837 & 1.33171 \\ \mathrm{C} & -4.56358 & 1.25824 & -0.46729 \\ \mathrm{H} & -4.51162 & 1.44409 & -2.61879 \\ \mathrm{H} & -4.38023 & 0.82088 & 1.63984 \\ \mathrm{H} & -5.35119 & 1.99588 & -0.33597 \\ \mathrm{H} & -0.22199 & -2.99331 & -2.12847 \\ \mathrm{C} & 3.49943 & -0.00885 & -0.65543 \\ \mathrm{H} & 4.13609 & -0.21495 & 0.21110 \\ \mathrm{H} & 2.94358 & -0.91130 & -0.92180 \\ \mathrm{H} & 4.12314 & 0.29761 & -1.50218 \\ \mathrm{C} & -0.72813 & 2.46936 & -0.13478 \\ \mathrm{H} & -0.84906 & 3.48907 & -0.51245 \\ \mathrm{H} & -1.25322 & 1.77135 & -0.78647 \\ \mathrm{H} & -1.15153 & 2.39675 & 0.87163\end{array}$

$\mathbf{I}^{\mathbf{B}}$

SCF Energy: -2316.80172485 hartrees

$\begin{array}{lrrr}\mathrm{C} & 0.48881 & -3.10434 & -1.22841 \\ \mathrm{C} & -0.19962 & -3.65218 & -0.19259 \\ \mathrm{C} & -0.40553 & -1.37772 & -0.02588 \\ \mathrm{~N} & 0.34632 & -1.72882 & -1.11666 \\ \mathrm{H} & 1.05966 & -3.56553 & -2.02080 \\ \mathrm{H} & -0.35244 & -4.68325 & 0.09023 \\ \mathrm{~N} & -0.73625 & -2.59081 & 0.52556 \\ \mathrm{Ni} & -0.74454 & 0.41073 & 0.63068\end{array}$




\begin{tabular}{lrrr}
$\mathrm{C}$ & 0.98782 & -0.76039 & -1.99767 \\
$\mathrm{H}$ & 2.06041 & -0.70353 & -1.79138 \\
$\mathrm{H}$ & 0.54550 & 0.21641 & -1.79962 \\
$\mathrm{H}$ & 0.81999 & -1.04101 & -3.04275 \\
$\mathrm{C}$ & -1.53922 & -2.73069 & 1.72933 \\
$\mathrm{H}$ & -1.00903 & -3.33531 & 2.47294 \\
$\mathrm{H}$ & -2.50337 & -3.20072 & 1.50320 \\
$\mathrm{H}$ & -1.70571 & -1.72849 & 2.13228 \\
$\mathrm{C}$ & -2.19247 & 1.16535 & -0.59709 \\
$\mathrm{C}$ & -3.40452 & 0.68875 & 0.30772 \\
$\mathrm{H}$ & -3.58593 & -0.38184 & 0.34292 \\
$\mathrm{H}$ & -3.53544 & 1.21409 & 1.24942 \\
$\mathrm{C}$ & -2.76367 & 2.57212 & -0.77451 \\
$\mathrm{H}$ & -2.46415 & 3.12120 & -1.66505 \\
$\mathrm{H}$ & -2.86257 & 3.17742 & 0.12349 \\
$\mathrm{C}$ & -2.82312 & 0.61430 & -1.87861 \\
$\mathrm{H}$ & -2.96909 & -0.46279 & -1.92722 \\
$\mathrm{H}$ & -2.52737 & 1.07809 & -2.81772 \\
$\mathrm{C}$ & -3.72991 & 1.41499 & -0.96150 \\
$\mathrm{C}$ & 0.80953 & 0.99563 & 1.72610 \\
$\mathrm{H}$ & 0.89162 & 0.36735 & 2.61668 \\
$\mathrm{C}$ & -0.21179 & 1.99151 & 1.70316 \\
$\mathrm{H}$ & -0.09024 & 2.90211 & 1.11304 \\
$\mathrm{H}$ & -0.80283 & 2.14048 & 2.61027 \\
$\mathrm{C}$ & 2.01928 & 0.97976 & 0.87821 \\
$\mathrm{C}$ & 2.29841 & 1.96822 & -0.08907 \\
$\mathrm{C}$ & 2.93959 & -0.08413 & 0.99546 \\
$\mathrm{C}$ & 3.42670 & 1.88620 & -0.90575 \\
$\mathrm{H}$ & 1.61711 & 2.80590 & -0.20578 \\
$\mathrm{C}$ & 4.06770 & -0.16861 & 0.18050 \\
$\mathrm{H}$ & 2.74555 & -0.86397 & 1.72968 \\
$\mathrm{C}$ & 4.31991 & 0.81520 & -0.78435 \\
$\mathrm{H}$ & 3.61224 & 2.66625 & -1.64146 \\
$\mathrm{H}$ & 4.75435 & -1.00463 & 0.29772 \\
$\mathrm{H}$ & 5.20032 & 0.75438 & -1.41916 \\
& & & \\
\hline & & &
\end{tabular}

$\mathbf{I}^{\mathbf{B}^{*}}$

SCF Energy: -2316.75569201 hartrees

$\begin{array}{lrrr}\mathrm{C} & 0.23970 & -3.30264 & -1.25571 \\ \mathrm{C} & -0.48932 & -3.93712 & -0.29900 \\ \mathrm{C} & -0.47427 & -1.71592 & 0.20924 \\ \mathrm{~N} & 0.23570 & -1.95550 & -0.93132 \\ \mathrm{H} & 0.74794 & -3.68565 & -2.12807 \\ \mathrm{H} & -0.74364 & -4.97925 & -0.17571 \\ \mathrm{~N} & -0.91725 & -2.95385 & 0.58143 \\ \mathrm{Ni} & -0.76911 & 0.04204 & 1.05051 \\ \mathrm{C} & 0.94256 & -0.92433 & -1.68728 \\ \mathrm{H} & 2.00932 & -0.93084 & -1.44844 \\ \mathrm{H} & 0.53838 & 0.04763 & -1.40948 \\ \mathrm{H} & 0.79466 & -1.09190 & -2.75785 \\ \mathrm{C} & -1.73139 & -3.20113 & 1.76318 \\ \mathrm{H} & -1.15090 & -3.72559 & 2.52956 \\ \mathrm{H} & -2.60869 & -3.79909 & 1.49744 \\ \mathrm{H} & -2.05958 & -2.23504 & 2.15422\end{array}$




$\begin{array}{lrrr}\mathrm{C} & -2.10978 & 0.96291 & -0.24322 \\ \mathrm{C} & -3.46089 & 1.65744 & 0.03844 \\ \mathrm{H} & -4.29840 & 1.01403 & 0.31694 \\ \mathrm{H} & -3.42610 & 2.58506 & 0.61294 \\ \mathrm{C} & -1.66859 & 2.16954 & -1.14417 \\ \mathrm{H} & -0.89805 & 1.96801 & -1.89100 \\ \mathrm{H} & -1.53187 & 3.12776 & -0.63952 \\ \mathrm{C} & -2.71111 & 0.25270 & -1.49377 \\ \mathrm{H} & -3.49682 & -0.48222 & -1.30530 \\ \mathrm{H} & -2.00547 & -0.06690 & -2.26388 \\ \mathrm{C} & -3.09539 & 1.73271 & -1.45607 \\ \mathrm{C} & 0.87051 & 0.96928 & 2.02528 \\ \mathrm{H} & 1.15872 & 0.28612 & 2.82788 \\ \mathrm{C} & -0.35652 & 1.67004 & 2.17408 \\ \mathrm{H} & -0.54138 & 2.59175 & 1.62544 \\ \mathrm{H} & -0.85640 & 1.62579 & 3.14246 \\ \mathrm{C} & 1.89515 & 1.18543 & 1.01251 \\ \mathrm{C} & 1.76459 & 2.14528 & -0.02172 \\ \mathrm{C} & 3.06540 & 0.38614 & 1.00675 \\ \mathrm{C} & 2.73337 & 2.27252 & -1.01530 \\ \mathrm{H} & 0.88755 & 2.78367 & -0.04906 \\ \mathrm{C} & 4.03249 & 0.51755 & 0.01509 \\ \mathrm{H} & 3.19755 & -0.35482 & 1.79345 \\ \mathrm{C} & 3.87244 & 1.45687 & -1.01613 \\ \mathrm{H} & 2.59970 & 3.01702 & -1.79759 \\ \mathrm{H} & 4.91852 & -0.11346 & 0.04225 \\ \mathrm{H} & 4.62660 & 1.56054 & -1.79174\end{array}$

\section{$\mathbf{I}^{\mathrm{C}}$}

SCF Energy: -2316.82493866 hartrees

$\begin{array}{lrrr}\mathrm{C} & -0.09193 & 2.35996 & 0.60200 \\ \mathrm{C} & -0.32541 & 1.39715 & 1.53151 \\ \mathrm{C} & -0.13263 & 0.36375 & -0.49133 \\ \mathrm{~N} & 0.02776 & 1.71268 & -0.62013 \\ \mathrm{H} & 0.00280 & 3.43106 & 0.69973 \\ \mathrm{H} & -0.46738 & 1.46181 & 2.60000 \\ \mathrm{~N} & -0.34287 & 0.18701 & 0.84846 \\ \mathrm{Ni} & -0.12132 & -0.96883 & -1.86532 \\ \mathrm{C} & 1.43108 & -1.65328 & -2.32974 \\ \mathrm{C} & 2.53932 & -1.24103 & -3.32157 \\ \mathrm{H} & 2.36262 & -1.71664 & -4.30061 \\ \mathrm{H} & 2.71142 & -0.17113 & -3.49038 \\ \mathrm{C} & 2.36120 & -2.76765 & -1.81049 \\ \mathrm{H} & 2.38636 & -2.95878 & -0.73080 \\ \mathrm{H} & 2.13011 & -3.71800 & -2.31995 \\ \mathrm{C} & 3.51988 & -2.02834 & -2.46455 \\ \mathrm{C} & 4.82016 & -1.91422 & -2.19657 \\ \mathrm{H} & 5.29773 & -2.52233 & -1.43093 \\ \mathrm{H} & 5.45028 & -1.20741 & -2.73269 \\ \mathrm{C} & 0.25746 & 2.38507 & -1.89241 \\ \mathrm{H} & 0.53792 & 1.62717 & -2.62544 \\ \mathrm{H} & -0.65343 & 2.88574 & -2.23123 \\ \mathrm{H} & 1.06949 & 3.11113 & -1.78365\end{array}$




$\begin{array}{lrrr}\mathrm{C} & -0.58723 & -1.10940 & 1.46105 \\ \mathrm{H} & -1.63681 & -1.20536 & 1.76055 \\ \mathrm{H} & -0.35298 & -1.87884 & 0.72220 \\ \mathrm{H} & 0.05469 & -1.23839 & 2.33839 \\ \mathrm{C} & -2.16120 & -0.85314 & -2.15027 \\ \mathrm{H} & -2.66194 & -1.13626 & -1.22337 \\ \mathrm{C} & -1.55101 & -1.87782 & -2.91218 \\ \mathrm{H} & -1.40009 & -1.77056 & -3.98527 \\ \mathrm{H} & -1.65900 & -2.91191 & -2.58642 \\ \mathrm{C} & -2.52793 & 0.48243 & -2.65423 \\ \mathrm{C} & -2.22342 & 0.92540 & -3.95843 \\ \mathrm{C} & -3.17639 & 1.39557 & -1.79652 \\ \mathrm{C} & -2.54689 & 2.21630 & -4.37796 \\ \mathrm{H} & -1.72112 & 0.25403 & -4.64856 \\ \mathrm{C} & -3.49820 & 2.68678 & -2.21257 \\ \mathrm{H} & -3.41231 & 1.08331 & -0.78074 \\ \mathrm{C} & -3.18247 & 3.11115 & -3.50937 \\ \mathrm{H} & -2.29984 & 2.52685 & -5.39095 \\ \mathrm{H} & -3.99599 & 3.36553 & -1.52329 \\ \mathrm{H} & -3.43435 & 4.11596 & -3.83872\end{array}$

$\mathbf{I}^{\mathrm{D}}$

SCF Energy: -2316.8420742 hartrees

$\begin{array}{lrrr}\mathrm{C} & 1.45564 & -1.20885 & 2.88126 \\ \mathrm{C} & 0.10298 & -1.17530 & 3.02763 \\ \mathrm{C} & 0.60987 & 0.01663 & 1.15586 \\ \mathrm{~N} & 1.74312 & -0.47545 & 1.74010 \\ \mathrm{H} & 2.22166 & -1.67830 & 3.48056 \\ \mathrm{H} & -0.53854 & -1.61694 & 3.77568 \\ \mathrm{~N} & -0.38947 & -0.42040 & 1.97699 \\ \mathrm{Ni} & 0.38894 & 0.92015 & -0.57442 \\ \mathrm{C} & 0.05103 & 1.71717 & -2.27691 \\ \mathrm{C} & -1.30309 & 2.49157 & -2.36399 \\ \mathrm{H} & -1.84737 & 2.28248 & -3.30264 \\ \mathrm{H} & -2.01939 & 2.40804 & -1.53450 \\ \mathrm{C} & 0.80226 & 3.02046 & -2.68739 \\ \mathrm{H} & 1.67593 & 3.33494 & -2.10070 \\ \mathrm{H} & 1.09065 & 3.02056 & -3.75312 \\ \mathrm{C} & -0.49259 & 3.77743 & -2.43047 \\ \mathrm{C} & -0.77968 & 5.05697 & -2.19193 \\ \mathrm{H} & -0.01224 & 5.82845 & -2.22654 \\ \mathrm{H} & -1.79236 & 5.37824 & -1.95370 \\ \mathrm{C} & 0.27771 & -0.54046 & -1.85903 \\ \mathrm{C} & 0.23492 & 0.41199 & -3.04917 \\ \mathrm{H} & -0.55130 & 0.19411 & -3.79352 \\ \mathrm{H} & 1.19903 & 0.40402 & -3.57421 \\ \mathrm{H} & 1.21214 & -1.10386 & -1.74857 \\ \mathrm{C} & -0.90197 & -1.34689 & -1.48278 \\ \mathrm{C} & -0.73980 & -2.51024 & -0.69820 \\ \mathrm{C} & -2.21937 & -0.99528 & -1.84969 \\ \mathrm{C} & -1.83042 & -3.28215 & -0.30031 \\ \mathrm{H} & 0.26459 & -2.80075 & -0.39531 \\ \mathrm{C} & -3.31245 & -1.76650 & -1.45213 \\ \mathrm{H} & -2.38534 & -0.09884 & -2.43913\end{array}$




$\begin{array}{lrrc}\mathrm{C} & -3.12973 & -2.91491 & -0.67282 \\ \mathrm{H} & -1.66744 & -4.17616 & 0.29822 \\ \mathrm{H} & -4.31460 & -1.46865 & -1.75289 \\ \mathrm{H} & -3.98225 & -3.51702 & -0.36881 \\ \mathrm{C} & 3.08230 & -0.26550 & 1.20604 \\ \mathrm{H} & 3.69031 & 0.31082 & 1.91107 \\ \mathrm{H} & 3.56618 & -1.22762 & 1.00901 \\ \mathrm{H} & 2.98266 & 0.28863 & 0.27004 \\ \mathrm{C} & -1.80842 & -0.19817 & 1.71676 \\ \mathrm{H} & -2.27942 & 0.27347 & 2.58475 \\ \mathrm{H} & -1.89191 & 0.45634 & 0.84779 \\ \mathrm{H} & -2.30027 & -1.14658 & 1.48499\end{array}$

$\mathbf{I}^{\mathbf{E}}$

SCF Energy: -2316.83600449 hartrees

$\begin{array}{lrrr}\mathrm{C} & -0.05046 & -3.78090 & 1.10326 \\ \mathrm{C} & -1.26661 & -3.66757 & 1.70096 \\ \mathrm{C} & -0.88188 & -1.71800 & 0.56476 \\ \mathrm{~N} & 0.16615 & -2.59718 & 0.41546 \\ \mathrm{H} & 0.66376 & -4.59105 & 1.10361 \\ \mathrm{H} & -1.81718 & -4.36220 & 2.31811 \\ \mathrm{~N} & -1.75780 & -2.41470 & 1.36526 \\ \mathrm{Ni} & -1.02004 & 0.02375 & -0.10898 \\ \mathrm{C} & 1.87516 & 1.88574 & -0.80798 \\ \mathrm{C} & 2.17420 & 1.31968 & 0.59723 \\ \mathrm{H} & 2.32216 & 2.05742 & 1.39606 \\ \mathrm{H} & 1.43251 & 0.57984 & 0.93023 \\ \mathrm{C} & 3.10007 & 1.15876 & -1.39913 \\ \mathrm{H} & 2.84490 & 0.33712 & -2.08268 \\ \mathrm{H} & 3.84730 & 1.79877 & -1.88612 \\ \mathrm{C} & 3.44430 & 0.70851 & 0.01823 \\ \mathrm{C} & 4.48182 & 0.06595 & 0.55134 \\ \mathrm{H} & 5.31501 & -0.27668 & -0.05949 \\ \mathrm{H} & 4.53820 & -0.13284 & 1.62008 \\ \mathrm{C} & 0.53934 & 2.05673 & -1.49263 \\ \mathrm{C} & 1.41196 & 3.26430 & -1.16390 \\ \mathrm{H} & 1.06948 & 3.90978 & -0.35661 \\ \mathrm{H} & 1.90390 & 3.78587 & -1.98377 \\ \mathrm{H} & 0.53591 & 1.75896 & -2.54081 \\ \mathrm{C} & -0.77773 & 1.82783 & -0.82210 \\ \mathrm{C} & -1.90163 & 1.36917 & -1.57623 \\ \mathrm{C} & -1.03350 & 2.12347 & 0.55308 \\ \mathrm{C} & -3.21651 & 1.45750 & -1.07024 \\ \mathrm{H} & -1.75569 & 1.08425 & -2.61573 \\ \mathrm{C} & -2.35032 & 2.22279 & 1.04928 \\ \mathrm{H} & -0.21343 & 2.42891 & 1.19591 \\ \mathrm{C} & -3.44683 & 1.92842 & 0.22840 \\ \mathrm{H} & -4.04820 & 1.15904 & -1.70337 \\ \mathrm{H} & -2.50274 & 2.52304 & 2.08299 \\ \mathrm{H} & -4.46055 & 2.02193 & 0.60890 \\ \mathrm{C} & 1.37403 & -2.28540 & -0.33437 \\ \mathrm{H} & 2.20131 & -2.02907 & 0.33522 \\ \mathrm{H} & 1.65727 & -3.13885 & -0.95916 \\ \mathrm{H} & 1.15613 & -1.42239 & -0.96804 \\ & & & \end{array}$




$\begin{array}{llll}\mathrm{C} & -3.04061 & -1.88755 & 1.80280 \\ \mathrm{H} & -3.06449 & -1.79604 & 2.89457 \\ \mathrm{H} & -3.16639 & -0.89938 & 1.35444 \\ \mathrm{H} & -3.85427 & -2.54379 & 1.47450\end{array}$

\section{$\mathbf{I}^{\mathbf{F}}$}

SCF Energy: -2316.80262907 hartrees

$\begin{array}{lrrr}\mathrm{C} & 1.21137 & -1.97839 & 1.49056 \\ \mathrm{C} & 0.30111 & -1.54588 & 2.40194 \\ \mathrm{C} & -0.09780 & -0.42826 & 0.45254 \\ \mathrm{~N} & 0.95036 & -1.29317 & 0.31156 \\ \mathrm{H} & 2.00553 & -2.70538 & 1.57327 \\ \mathrm{H} & 0.14133 & -1.82567 & 3.43262 \\ \mathrm{~N} & -0.48696 & -0.60383 & 1.75133 \\ \mathrm{Ni} & -0.84739 & 0.70274 & -0.91375 \\ \mathrm{C} & -2.45297 & -0.01240 & -2.82318 \\ \mathrm{C} & -1.66067 & 1.42008 & -2.55657 \\ \mathrm{C} & -1.41919 & 0.52127 & -3.76651 \\ \mathrm{C} & -3.17285 & 1.29110 & -2.62330 \\ \mathrm{C} & -1.98129 & -0.74229 & -1.63274 \\ \mathrm{H} & -1.38755 & -1.63836 & -1.84650 \\ \mathrm{H} & -2.76260 & -0.97266 & -0.89820 \\ \mathrm{H} & -1.78398 & 0.87911 & -4.73448 \\ \mathrm{H} & -0.45962 & 0.00620 & -3.83031 \\ \mathrm{H} & -3.67748 & 1.69626 & -3.50625 \\ \mathrm{H} & -3.73364 & 1.43774 & -1.69953 \\ \mathrm{C} & -0.41502 & 2.97540 & -0.43739 \\ \mathrm{C} & 0.69319 & 2.34406 & 0.03696 \\ \mathrm{H} & -0.46248 & 3.40594 & -1.42860 \\ \mathrm{H} & -1.25061 & 3.19487 & 0.22167 \\ \mathrm{H} & 0.71825 & 2.06665 & 1.08862 \\ \mathrm{C} & 1.92629 & 2.05511 & -0.71335 \\ \mathrm{C} & 3.10313 & 1.73364 & -0.01305 \\ \mathrm{C} & 1.95731 & 2.03073 & -2.12229 \\ \mathrm{C} & 4.28056 & 1.41479 & -0.69249 \\ \mathrm{H} & 3.08970 & 1.73167 & 1.07522 \\ \mathrm{C} & 3.13165 & 1.70539 & -2.79970 \\ \mathrm{H} & 1.04247 & 2.22020 & -2.67654 \\ \mathrm{C} & 4.29987 & 1.39765 & -2.09088 \\ \mathrm{H} & 5.18096 & 1.17665 & -0.13104 \\ \mathrm{H} & 3.13357 & 1.68125 & -3.88677 \\ \mathrm{H} & 5.21337 & 1.14504 & -2.62324 \\ \mathrm{C} & 1.71305 & -1.45339 & -0.92211 \\ \mathrm{H} & 1.84896 & -2.51817 & -1.13476 \\ \mathrm{H} & 1.14762 & -0.98577 & -1.72921 \\ \mathrm{H} & 2.68459 & -0.95831 & -0.84140 \\ \mathrm{C} & -1.59867 & 0.11166 & 2.35981 \\ \mathrm{H} & -2.05065 & 0.73849 & 1.58787 \\ \mathrm{H} & -2.34651 & -0.59425 & 2.73556 \\ \mathrm{H} & -1.24690 & 0.74159 & 3.18422\end{array}$

$\mathbf{I}^{\mathbf{G}}$

SCF Energy: -2316.8350428 hartrees

$\begin{array}{llll}\text { C } & 2.74247 & -1.71548 & 1.25264\end{array}$ 


$\begin{array}{lrrr}\mathrm{C} & 3.45838 & -0.57027 & 1.10372 \\ \mathrm{C} & 1.25122 & -0.02062 & 0.96529 \\ \mathrm{~N} & 1.40337 & -1.35998 & 1.17574 \\ \mathrm{H} & 3.06153 & -2.73671 & 1.39743 \\ \mathrm{H} & 4.52351 & -0.39323 & 1.10892 \\ \mathrm{~N} & 2.53284 & 0.45124 & 0.92709 \\ \mathrm{Ni} & -0.36502 & 0.91510 & 0.60332 \\ \mathrm{C} & -1.99696 & 1.85378 & -0.14743 \\ \mathrm{C} & -2.98524 & 1.03245 & 0.68331 \\ \mathrm{H} & -4.02788 & 1.39436 & 0.72161 \\ \mathrm{H} & -2.94963 & -0.05883 & 0.60509 \\ \mathrm{C} & -2.00066 & 2.99338 & 0.86933 \\ \mathrm{H} & -1.10355 & 3.61564 & 0.94986 \\ \mathrm{H} & -2.91799 & 3.60469 & 0.93599 \\ \mathrm{C} & -2.02955 & 1.69657 & 1.66770 \\ \mathrm{C} & -1.12025 & 1.15804 & 2.57038 \\ \mathrm{H} & -0.47061 & 1.81827 & 3.14031 \\ \mathrm{H} & -1.30464 & 0.17710 & 3.00277 \\ \mathrm{C} & 0.29565 & -2.30769 & 1.20050 \\ \mathrm{H} & -0.62244 & -1.74969 & 1.39001 \\ \mathrm{H} & 0.20799 & -2.81615 & 0.23648 \\ \mathrm{H} & 0.45482 & -3.03750 & 2.00037 \\ \mathrm{C} & 2.86856 & 1.85546 & 0.74763 \\ \mathrm{H} & 3.19345 & 2.30211 & 1.69388 \\ \mathrm{H} & 3.66546 & 1.95760 & 0.00435 \\ \mathrm{H} & 1.97287 & 2.37023 & 0.39304 \\ \mathrm{C} & -0.25081 & 0.86747 & -1.39672 \\ \mathrm{H} & 0.67448 & 1.34474 & -1.73703 \\ \mathrm{C} & -1.49756 & 1.75362 & -1.57298 \\ \mathrm{H} & -2.25315 & 1.36075 & -2.27298 \\ \mathrm{H} & -1.21563 & 2.75172 & -1.93132 \\ \mathrm{C} & -0.30808 & -0.54851 & -1.80766 \\ \mathrm{C} & -1.51034 & -1.28879 & -1.87369 \\ \mathrm{C} & 0.88377 & -1.24860 & -2.11275 \\ \mathrm{C} & -1.51844 & -2.64347 & -2.21353 \\ \mathrm{H} & -2.45216 & -0.79914 & -1.64318 \\ \mathrm{C} & 0.87808 & -2.59968 & -2.45121 \\ \mathrm{H} & 1.82973 & -0.71139 & -2.06689 \\ \mathrm{C} & -0.32613 & -3.31621 & -2.50126 \\ \mathrm{H} & -2.46584 & -3.17748 & -2.25327 \\ \mathrm{H} & 1.81760 & -3.09875 & -2.68131 \\ \mathrm{H} & -0.33385 & -4.36970 & -2.76892\end{array}$

$\mathbf{I}^{\mathrm{H}}$

SCF Energy: -2316.85273225 hartrees

$\begin{array}{lrrr}\mathrm{C} & 3.32718 & 2.02768 & -0.03416 \\ \mathrm{C} & 2.72847 & 1.50848 & 1.07004 \\ \mathrm{C} & 1.63909 & 0.66250 & -0.75005 \\ \mathrm{~N} & 2.65208 & 1.50706 & -1.12937 \\ \mathrm{H} & 4.15332 & 2.71593 & -0.13424 \\ \mathrm{H} & 2.93366 & 1.65425 & 2.12022 \\ \mathrm{~N} & 1.70501 & 0.68838 & 0.61976 \\ \mathrm{Ni} & 0.48075 & -0.38205 & -1.83839 \\ \mathrm{C} & -0.57658 & -1.50858 & -3.07631\end{array}$




$\begin{array}{lrrr}\mathrm{H} & -1.15780 & -0.91573 & -3.78714 \\ \mathrm{C} & -1.00047 & -1.62453 & -1.74837 \\ \mathrm{H} & 0.04795 & -2.28530 & -3.52557 \\ \mathrm{C} & -0.82172 & -2.76321 & -0.72782 \\ \mathrm{C} & -2.28605 & -1.13048 & -1.06043 \\ \mathrm{C} & -2.09468 & -2.25360 & -0.02508 \\ \mathrm{H} & 0.09527 & -2.71036 & -0.12965 \\ \mathrm{H} & -0.90456 & -3.77455 & -1.15003 \\ \mathrm{H} & -2.23955 & -0.11359 & -0.65058 \\ \mathrm{H} & -3.19239 & -1.22214 & -1.67516 \\ \mathrm{C} & -2.33586 & -2.10612 & 1.47249 \\ \mathrm{C} & -3.16106 & -3.06858 & 0.62721 \\ \mathrm{H} & -2.85050 & -1.18531 & 1.74654 \\ \mathrm{H} & -2.98128 & -4.13382 & 0.76414 \\ \mathrm{H} & -4.20263 & -2.80853 & 0.44605 \\ \mathrm{C} & -1.35806 & -2.56066 & 2.49665 \\ \mathrm{C} & -0.61984 & -3.75100 & 2.37068 \\ \mathrm{C} & -1.12715 & -1.76277 & 3.63063 \\ \mathrm{C} & 0.32091 & -4.11940 & 3.33454 \\ \mathrm{H} & -0.77706 & -4.39477 & 1.51030 \\ \mathrm{C} & -0.18965 & -2.12977 & 4.59855 \\ \mathrm{H} & -1.69205 & -0.84054 & 3.75310 \\ \mathrm{C} & 0.54510 & -3.30998 & 4.45291 \\ \mathrm{H} & 0.88129 & -5.04297 & 3.21041 \\ \mathrm{H} & -0.03497 & -1.49366 & 5.46710 \\ \mathrm{H} & 1.27704 & -3.59886 & 5.20277 \\ \mathrm{C} & 0.84150 & -0.10150 & 1.48815 \\ \mathrm{H} & -0.03659 & -0.40146 & 0.91642 \\ \mathrm{H} & 1.35445 & -1.00013 & 1.84437 \\ \mathrm{H} & 0.52860 & 0.49707 & 2.34808 \\ \mathrm{C} & 2.98579 & 1.80091 & -2.51499 \\ \mathrm{H} & 2.23371 & 1.32977 & -3.15208 \\ \mathrm{H} & 2.97794 & 2.88262 & -2.68645 \\ \mathrm{H} & 3.97322 & 1.39877 & -2.76695 \\ & & & \end{array}$

\section{TS $^{\mathrm{BC}}$}

SCF Energy: -2316.78653575 hartrees

$\begin{array}{llll}\mathrm{C} & 0.33163 & 2.36459 & 0.62017 \\ \mathrm{C} & 0.20452 & 1.40560 & 1.57427 \\ \mathrm{C} & 0.15577 & 0.35724 & -0.46111 \\ \mathrm{~N} & 0.30662 & 1.71196 & -0.60468 \\ \mathrm{H} & 0.43487 & 3.43639 & 0.70380 \\ \mathrm{H} & 0.18037 & 1.47710 & 2.65171 \\ \mathrm{~N} & 0.10332 & 0.19380 & 0.90139 \\ \mathrm{Ni} & -0.07261 & -0.96659 & -1.84175 \\ \mathrm{C} & 1.46091 & -1.85055 & -2.49060 \\ \mathrm{C} & 2.35421 & -1.59135 & -3.71659 \\ \mathrm{H} & 2.20882 & -2.27691 & -4.55295 \\ \mathrm{H} & 2.50812 & -0.55585 & -4.01569 \\ \mathrm{C} & 2.13148 & -3.20229 & -2.19429 \\ \mathrm{H} & 2.09432 & -3.56990 & -1.17003 \\ \mathrm{H} & 1.97355 & -3.97635 & -2.94708 \\ \mathrm{C} & 3.13029 & -2.12791 & -2.54143 \\ \mathrm{C} & 3.14636 & -1.17030 & -1.52578\end{array}$




$\begin{array}{lrrr}\mathrm{H} & 3.12843 & -1.45924 & -0.47912 \\ \mathrm{H} & 3.30936 & -0.11937 & -1.74480 \\ \mathrm{C} & 0.37396 & 2.38330 & -1.89595 \\ \mathrm{H} & 0.49372 & 1.61764 & -2.66397 \\ \mathrm{H} & -0.55152 & 2.93053 & -2.09537 \\ \mathrm{H} & 1.22815 & 3.06898 & -1.91599 \\ \mathrm{C} & -0.07980 & -1.09626 & 1.54516 \\ \mathrm{H} & -1.04188 & -1.13508 & 2.06831 \\ \mathrm{H} & -0.06722 & -1.85984 & 0.76354 \\ \mathrm{H} & 0.72830 & -1.28706 & 2.26044 \\ \mathrm{C} & -2.03716 & -0.89256 & -2.23495 \\ \mathrm{H} & -2.59370 & -1.22717 & -1.35621 \\ \mathrm{C} & -1.38426 & -1.88404 & -3.02521 \\ \mathrm{H} & -1.20882 & -1.72461 & -4.09040 \\ \mathrm{H} & -1.54074 & -2.93340 & -2.76883 \\ \mathrm{C} & -2.40010 & 0.46389 & -2.68616 \\ \mathrm{C} & -2.12066 & 0.95251 & -3.98054 \\ \mathrm{C} & -3.02883 & 1.35288 & -1.78672 \\ \mathrm{C} & -2.43783 & 2.26120 & -4.34699 \\ \mathrm{H} & -1.64568 & 0.29829 & -4.70590 \\ \mathrm{C} & -3.34755 & 2.66011 & -2.15058 \\ \mathrm{H} & -3.24707 & 1.00570 & -0.77836 \\ \mathrm{C} & -3.04897 & 3.13098 & -3.43604 \\ \mathrm{H} & -2.20910 & 2.60465 & -5.35403 \\ \mathrm{H} & -3.82947 & 3.31674 & -1.42889 \\ \mathrm{H} & -3.29800 & 4.14910 & -3.72455\end{array}$

\section{TS $^{\mathrm{CD}}$}

SCF Energy: -2316.80844041 hartrees

$\begin{array}{lrrr}\mathrm{C} & 1.16165 & -1.13462 & 2.92026 \\ \mathrm{C} & -0.02171 & -0.49424 & 3.10908 \\ \mathrm{C} & 0.54790 & -0.22838 & 0.91981 \\ \mathrm{~N} & 1.49648 & -0.96207 & 1.58347 \\ \mathrm{H} & 1.79196 & -1.67663 & 3.60927 \\ \mathrm{H} & -0.62549 & -0.36937 & 3.99543 \\ \mathrm{~N} & -0.38057 & 0.05286 & 1.88544 \\ \mathrm{Ni} & 0.51906 & 0.24932 & -0.88089 \\ \mathrm{C} & 0.77557 & 1.27346 & -2.41793 \\ \mathrm{C} & -0.03259 & 2.47812 & -2.96849 \\ \mathrm{H} & -0.72619 & 2.21407 & -3.78886 \\ \mathrm{H} & -0.56574 & 3.11796 & -2.25601 \\ \mathrm{C} & 1.87595 & 1.56817 & -3.46101 \\ \mathrm{H} & 2.92438 & 1.45069 & -3.16222 \\ \mathrm{H} & 1.72538 & 1.04280 & -4.42279 \\ \mathrm{C} & 1.29288 & 2.97112 & -3.52709 \\ \mathrm{C} & 1.80315 & 4.17636 & -3.77504 \\ \mathrm{H} & 2.83745 & 4.30166 & -4.08942 \\ \mathrm{H} & 1.20476 & 5.07922 & -3.66954 \\ \mathrm{C} & -0.03533 & -0.38931 & -2.79940 \\ \mathrm{H} & -0.81305 & 0.00110 & -3.45603 \\ \mathrm{C} & -0.51124 & -1.18246 & -1.65157 \\ \mathrm{H} & -0.02389 & -2.14882 & -1.49840 \\ \mathrm{C} & -1.91534 & -1.15149 & -1.18296 \\ \mathrm{C} & -2.80763 & -0.11415 & -1.52125\end{array}$




$\begin{array}{lrrr}\mathrm{C} & -2.37881 & -2.15293 & -0.30762 \\ \mathrm{C} & -4.10357 & -0.08193 & -1.00647 \\ \mathrm{H} & -2.47444 & 0.68597 & -2.17612 \\ \mathrm{C} & -3.67299 & -2.12061 & 0.21237 \\ \mathrm{H} & -1.70378 & -2.95851 & -0.02519 \\ \mathrm{C} & -4.54571 & -1.08204 & -0.13267 \\ \mathrm{H} & -4.77037 & 0.73129 & -1.28375 \\ \mathrm{H} & -4.00274 & -2.90797 & 0.88644 \\ \mathrm{H} & -5.55591 & -1.05588 & 0.26765 \\ \mathrm{H} & 0.75773 & -0.88200 & -3.36210 \\ \mathrm{C} & 2.68240 & -1.51185 & 0.94448 \\ \mathrm{H} & 2.54169 & -2.57241 & 0.70769 \\ \mathrm{H} & 2.85705 & -0.95782 & 0.01883 \\ \mathrm{H} & 3.54687 & -1.39866 & 1.60590 \\ \mathrm{C} & -1.60061 & 0.81288 & 1.64362 \\ \mathrm{H} & -1.46937 & 1.38063 & 0.72089 \\ \mathrm{H} & -2.45719 & 0.14328 & 1.52674 \\ \mathrm{H} & -1.77346 & 1.50179 & 2.47613\end{array}$

TS $^{\text {CD' }}$

SCF Energy: -2316.79892845 hartrees

$\begin{array}{lrrr}\mathrm{C} & 1.10206 & -1.08935 & 2.97162 \\ \mathrm{C} & -0.13354 & -0.54307 & 3.11600 \\ \mathrm{C} & 0.51319 & -0.19717 & 0.95660 \\ \mathrm{~N} & 1.48214 & -0.87000 & 1.65411 \\ \mathrm{H} & 1.74042 & -1.59335 & 3.68176 \\ \mathrm{H} & -0.78137 & -0.47637 & 3.97721 \\ \mathrm{~N} & -0.47750 & -0.00497 & 1.88286 \\ \mathrm{Ni} & 0.56931 & 0.33943 & -0.82271 \\ \mathrm{C} & 0.86708 & 1.30886 & -2.39595 \\ \mathrm{C} & 0.10288 & 2.46867 & -3.07931 \\ \mathrm{H} & -0.42678 & 2.16594 & -4.00205 \\ \mathrm{H} & -0.57201 & 3.08591 & -2.47501 \\ \mathrm{C} & 2.09597 & 1.63115 & -3.27950 \\ \mathrm{H} & 3.09314 & 1.54156 & -2.83538 \\ \mathrm{H} & 2.10036 & 1.11598 & -4.25391 \\ \mathrm{C} & 1.48017 & 3.01442 & -3.41472 \\ \mathrm{C} & 1.98007 & 4.24220 & -3.55129 \\ \mathrm{H} & 3.04668 & 4.40869 & -3.68885 \\ \mathrm{H} & 1.33979 & 5.12197 & -3.52951 \\ \mathrm{C} & 0.02295 & -0.44045 & -2.82200 \\ \mathrm{H} & -0.78419 & 0.01277 & -3.39722 \\ \mathrm{C} & -0.42414 & -1.08512 & -1.58309 \\ \mathrm{H} & -0.04512 & -2.08556 & -1.36203 \\ \mathrm{C} & 2.72926 & -1.32392 & 1.05633 \\ \mathrm{H} & 2.64643 & -2.36510 & 0.72526 \\ \mathrm{H} & 2.94343 & -0.68968 & 0.19230 \\ \mathrm{H} & 3.54157 & -1.23637 & 1.78376 \\ \mathrm{C} & -1.73701 & 0.66208 & 1.58768 \\ \mathrm{H} & -1.60860 & 1.23077 & 0.66400 \\ \mathrm{H} & -2.54168 & -0.06905 & 1.45280 \\ \mathrm{H} & -1.99701 & 1.34435 & 2.40283 \\ \mathrm{H} & -1.48263 & -0.97737 & -1.32585 \\ \mathrm{C} & 1.01762 & -1.15570 & -3.66624\end{array}$




$\begin{array}{llll}\mathrm{C} & 0.87870 & -1.18417 & -5.06277 \\ \mathrm{C} & 2.14306 & -1.78090 & -3.09967 \\ \mathrm{C} & 1.82046 & -1.83196 & -5.86780 \\ \mathrm{H} & 0.02158 & -0.69608 & -5.52173 \\ \mathrm{C} & 3.08383 & -2.42826 & -3.89947 \\ \mathrm{H} & 2.28174 & -1.73434 & -2.02219 \\ \mathrm{C} & 2.92704 & -2.45993 & -5.29049 \\ \mathrm{H} & 1.68834 & -1.84335 & -6.94714 \\ \mathrm{H} & 3.94813 & -2.90210 & -3.43927 \\ \mathrm{H} & 3.66179 & -2.96215 & -5.91457\end{array}$

\section{TS $^{\mathrm{DE}}$}

SCF Energy: -2316.81616065 hartrees

$\begin{array}{lrrr}\mathrm{C} & 0.35303 & -3.65758 & 0.57427 \\ \mathrm{C} & -0.30105 & -3.19884 & 1.67294 \\ \mathrm{C} & -0.21622 & -1.47517 & 0.16393 \\ \mathrm{~N} & 0.39138 & -2.60676 & -0.33104 \\ \mathrm{H} & 0.77927 & -4.62631 & 0.35900 \\ \mathrm{H} & -0.55524 & -3.69102 & 2.60010 \\ \mathrm{~N} & -0.64704 & -1.88146 & 1.40866 \\ \mathrm{Ni} & -0.35988 & 0.24374 & -0.54457 \\ \mathrm{C} & 1.55257 & 1.58348 & -0.34982 \\ \mathrm{C} & 1.69044 & 1.28619 & 1.16895 \\ \mathrm{H} & 2.15072 & 2.13599 & 1.70132 \\ \mathrm{H} & 0.80936 & 0.96268 & 1.73150 \\ \mathrm{C} & 2.75092 & 0.66798 & -0.67002 \\ \mathrm{H} & 2.59720 & -0.10831 & -1.42527 \\ \mathrm{H} & 3.66573 & 1.23065 & -0.91970 \\ \mathrm{C} & 2.70369 & 0.21195 & 0.78625 \\ \mathrm{C} & 3.26619 & -0.79369 & 1.45091 \\ \mathrm{H} & 3.95067 & -1.48545 & 0.96364 \\ \mathrm{H} & 3.05220 & -0.96985 & 2.50312 \\ \mathrm{C} & 0.27827 & 2.10582 & -1.43658 \\ \mathrm{C} & 1.37184 & 2.96699 & -0.88641 \\ \mathrm{H} & 1.08761 & 3.73202 & -0.16290 \\ \mathrm{H} & 2.10495 & 3.32869 & -1.60909 \\ \mathrm{H} & 0.46345 & 1.74790 & -2.44906 \\ \mathrm{C} & -1.11839 & 2.07225 & -0.99157 \\ \mathrm{C} & -2.04739 & 1.21059 & -1.66865 \\ \mathrm{C} & -1.58687 & 2.75313 & 0.17797 \\ \mathrm{C} & -3.38365 & 1.11272 & -1.21776 \\ \mathrm{H} & -1.79078 & 0.79254 & -2.64047 \\ \mathrm{C} & -2.89947 & 2.62892 & 0.60085 \\ \mathrm{H} & -0.90203 & 3.38657 & 0.73511 \\ \mathrm{C} & -3.81452 & 1.80862 & -0.09771 \\ \mathrm{H} & -4.07526 & 0.47875 & -1.76798 \\ \mathrm{H} & -3.22910 & 3.17389 & 1.48296 \\ \mathrm{H} & -4.84279 & 1.72372 & 0.24383 \\ \mathrm{C} & 1.04143 & -2.66502 & -1.62724 \\ \mathrm{H} & 2.12778 & -2.75603 & -1.51171 \\ \mathrm{H} & 0.66505 & -3.51505 & -2.20709 \\ \mathrm{H} & 0.80857 & -1.73544 & -2.15207 \\ \mathrm{C} & -1.37472 & -1.02416 & 2.33171 \\ \mathrm{H} & -0.73486 & -0.72071 & 3.16874\end{array}$




$$
\begin{array}{lrrr}
\text { H } & -1.69910 & -0.13462 & 1.78539 \\
\text { H } & -2.25190 & -1.55198 & 2.72081 \\
& & & \\
\text { TS } & \\
\text { SCF } & \text { Energy: -2316.79515438 hartrees }
\end{array}
$$

\section{TS $^{\mathrm{BF}}$}

$\begin{array}{lrrr}\mathrm{C} & 0.76328 & 2.93656 & 1.17204 \\ \mathrm{C} & 0.17072 & 3.51443 & 0.09458 \\ \mathrm{C} & -0.33668 & 1.28498 & 0.04337 \\ \mathrm{~N} & 0.43811 & 1.58826 & 1.12763 \\ \mathrm{H} & 1.38070 & 3.35798 & 1.95138 \\ \mathrm{H} & 0.15979 & 4.53975 & -0.24444 \\ \mathrm{~N} & -0.48952 & 2.49489 & -0.58117 \\ \mathrm{Ni} & -0.92900 & -0.49863 & -0.52299 \\ \mathrm{C} & 0.95122 & 0.58867 & 2.05820 \\ \mathrm{H} & 1.98672 & 0.33664 & 1.81247 \\ \mathrm{H} & 0.33744 & -0.30794 & 1.96528 \\ \mathrm{H} & 0.89072 & 0.97409 & 3.08089 \\ \mathrm{C} & -1.24365 & 2.67477 & -1.81146 \\ \mathrm{H} & -0.68080 & 3.30632 & -2.50602 \\ \mathrm{H} & -2.21780 & 3.13662 & -1.61353 \\ \mathrm{H} & -1.39295 & 1.69021 & -2.25979 \\ \mathrm{C} & -2.12336 & -1.16069 & 0.82863 \\ \mathrm{C} & -3.07955 & -0.37945 & -0.67834 \\ \mathrm{H} & -3.14633 & 0.70357 & -0.77116 \\ \mathrm{H} & -3.18784 & -0.93529 & -1.60853 \\ \mathrm{C} & -3.09103 & -2.32608 & 0.69649 \\ \mathrm{H} & -3.35049 & -2.84833 & 1.61749 \\ \mathrm{H} & -2.97562 & -2.96911 & -0.17257 \\ \mathrm{C} & -3.04299 & -0.32443 & 1.70419 \\ \mathrm{H} & -2.88661 & 0.75245 & 1.70030 \\ \mathrm{H} & -3.30190 & -0.74173 & 2.67745 \\ \mathrm{C} & -3.71117 & -0.96252 & 0.50893 \\ \mathrm{C} & 0.54663 & -1.07595 & -1.76194 \\ \mathrm{H} & 0.59522 & -0.43400 & -2.64351 \\ \mathrm{C} & -0.49527 & -2.04230 & -1.68637 \\ \mathrm{H} & -0.36458 & -2.96868 & -1.12526 \\ \mathrm{H} & -1.15860 & -2.14940 & -2.54719 \\ \mathrm{C} & 1.79182 & -1.10457 & -0.96827 \\ \mathrm{C} & 2.09241 & -2.12289 & -0.03894 \\ \mathrm{C} & 2.72660 & -0.05353 & -1.09454 \\ \mathrm{C} & 3.25787 & -2.08500 & 0.72821 \\ \mathrm{H} & 1.40142 & -2.95076 & 0.08839 \\ \mathrm{C} & 3.88973 & -0.01259 & -0.32823 \\ \mathrm{H} & 2.51512 & 0.75251 & -1.79500 \\ \mathrm{C} & 4.16591 & -1.02862 & 0.59665 \\ \mathrm{H} & 3.45830 & -2.88814 & 1.43443 \\ \mathrm{H} & 4.58528 & 0.81516 & -0.45117 \\ \mathrm{H} & 5.07415 & -1.00144 & 1.19336\end{array}$

\section{TS $^{\mathrm{FG}}$}

SCF Energy: -2316.78151034 hartrees
$\begin{array}{lll}\text { C } & 2.92596 & -0.98854\end{array}$
0.18514
$\begin{array}{lll}\text { C } & 0.72821 & -0.47672\end{array}$
0.11733
$-0.19242$ 


$\begin{array}{lrrr}\mathrm{N} & 0.88792 & -1.81726 & 0.00157 \\ \mathrm{H} & 2.54629 & -3.16582 & 0.34694 \\ \mathrm{H} & 3.98318 & -0.79276 & 0.21631 \\ \mathrm{~N} & 1.99914 & 0.02068 & -0.10938 \\ \mathrm{Ni} & -0.87348 & 0.45603 & -0.58096 \\ \mathrm{C} & -2.67126 & 1.49503 & -0.93683 \\ \mathrm{C} & -3.77604 & 0.74935 & -0.21362 \\ \mathrm{H} & -4.78321 & 1.18009 & -0.23761 \\ \mathrm{H} & -3.76702 & -0.34000 & -0.27029 \\ \mathrm{C} & -2.71422 & 2.71871 & -0.04295 \\ \mathrm{H} & -1.80123 & 3.30920 & 0.04711 \\ \mathrm{H} & -3.62722 & 3.32399 & -0.05590 \\ \mathrm{C} & -2.77085 & 1.40963 & 0.68760 \\ \mathrm{C} & -1.57648 & 0.72472 & 1.23235 \\ \mathrm{H} & -0.93826 & 1.36006 & 1.85562 \\ \mathrm{H} & -1.78979 & -0.21820 & 1.74767 \\ \mathrm{C} & -0.20184 & -2.78617 & -0.04437 \\ \mathrm{H} & -1.14212 & -2.24101 & 0.04621 \\ \mathrm{H} & -0.19372 & -3.32220 & -0.99658 \\ \mathrm{H} & -0.10110 & -3.48940 & 0.78810 \\ \mathrm{C} & 2.33205 & 1.42759 & -0.27551 \\ \mathrm{H} & 2.95673 & 1.76843 & 0.55620 \\ \mathrm{H} & 2.86226 & 1.58901 & -1.22036 \\ \mathrm{H} & 1.39867 & 1.99445 & -0.28498 \\ \mathrm{C} & -0.61853 & 0.32458 & -2.88415 \\ \mathrm{H} & 0.37118 & 0.76848 & -2.97534 \\ \mathrm{C} & -1.72845 & 1.19776 & -2.76300 \\ \mathrm{H} & -2.69163 & 0.87948 & -3.14893 \\ \mathrm{H} & -1.54020 & 2.26043 & -2.87234 \\ \mathrm{C} & -0.72398 & -1.10470 & -3.14182 \\ \mathrm{C} & -1.94455 & -1.81657 & -3.03870 \\ \mathrm{C} & 0.42758 & -1.85625 & -3.47840 \\ \mathrm{C} & -2.00870 & -3.18753 & -3.28659 \\ \mathrm{H} & -2.84868 & -1.28916 & -2.74577 \\ \mathrm{C} & 0.36212 & -3.22632 & -3.71967 \\ \mathrm{H} & 1.38562 & -1.34352 & -3.54536 \\ \mathrm{C} & -0.85894 & -3.90986 & -3.62824 \\ \mathrm{H} & -2.96526 & -3.69947 & -3.20266 \\ \mathrm{H} & 1.26917 & -3.76756 & -3.98162 \\ \mathrm{H} & -0.91185 & -4.97819 & -3.82065 \\ & & & \end{array}$

TS $^{\text {FG' }}$

SCF Energy: -2316.7678593 hartrees

$\begin{array}{lrrr}\mathrm{C} & 2.05492 & -2.27140 & -0.18874 \\ \mathrm{C} & 2.81609 & -1.15832 & -0.02771 \\ \mathrm{C} & 0.67134 & -0.46856 & -0.37719 \\ \mathrm{~N} & 0.75188 & -1.83443 & -0.39356 \\ \mathrm{H} & 2.31700 & -3.31855 & -0.16473 \\ \mathrm{H} & 3.87198 & -1.04440 & 0.16695 \\ \mathrm{~N} & 1.95819 & -0.07087 & -0.14134 \\ \mathrm{Ni} & -0.85199 & 0.58319 & -0.64307 \\ \mathrm{C} & -2.71966 & 1.78934 & -0.75861 \\ \mathrm{C} & -3.78361 & 1.03470 & 0.00989 \\ \mathrm{H} & -4.75188 & 1.53234 & 0.13653\end{array}$




$\begin{array}{lrrr}\mathrm{H} & -3.87076 & -0.03604 & -0.17761 \\ \mathrm{C} & -2.54664 & 2.89150 & 0.27053 \\ \mathrm{H} & -1.57954 & 3.39516 & 0.31662 \\ \mathrm{H} & -3.39567 & 3.56650 & 0.43131 \\ \mathrm{C} & -2.64111 & 1.51132 & 0.85050 \\ \mathrm{C} & -1.47338 & 0.67557 & 1.22698 \\ \mathrm{H} & -0.76284 & 1.18148 & 1.89105 \\ \mathrm{H} & -1.75128 & -0.29297 & 1.65858 \\ \mathrm{C} & -0.38939 & -2.70061 & -0.64838 \\ \mathrm{H} & -0.31570 & -3.60131 & -0.03189 \\ \mathrm{H} & -1.29597 & -2.15086 & -0.38557 \\ \mathrm{H} & -0.43400 & -2.98400 & -1.70585 \\ \mathrm{C} & 2.36937 & 1.32248 & -0.05553 \\ \mathrm{H} & 1.46798 & 1.93335 & 0.02534 \\ \mathrm{H} & 2.99468 & 1.47507 & 0.82969 \\ \mathrm{H} & 2.92533 & 1.61684 & -0.95228 \\ \mathrm{C} & -0.62213 & 0.86542 & -2.76237 \\ \mathrm{H} & 0.23453 & 1.52545 & -2.89495 \\ \mathrm{C} & -1.91305 & 1.46251 & -2.71533 \\ \mathrm{H} & -1.96177 & 2.54123 & -2.82140 \\ \mathrm{H} & -0.51452 & -0.12033 & -3.20961 \\ \mathrm{C} & -3.11435 & 0.74679 & -3.21687 \\ \mathrm{C} & -4.13974 & 1.45846 & -3.85520 \\ \mathrm{C} & -3.25060 & -0.64415 & -3.07771 \\ \mathrm{C} & -5.26379 & 0.79878 & -4.35899 \\ \mathrm{H} & -4.05716 & 2.53880 & -3.95181 \\ \mathrm{C} & -4.37324 & -1.30543 & -3.57376 \\ \mathrm{H} & -2.47712 & -1.19860 & -2.55211 \\ \mathrm{C} & -5.38567 & -0.58652 & -4.22056 \\ \mathrm{H} & -6.04627 & 1.36826 & -4.85477 \\ \mathrm{H} & -4.46479 & -2.38219 & -3.45037 \\ \mathrm{H} & -6.26200 & -1.10118 & -4.60653\end{array}$

\section{$\mathbf{T S}^{\mathrm{GH}}$}

SCF Energy: -2316.80997299 hartrees

$\begin{array}{lrrr}\mathrm{C} & 2.79018 & -1.83624 & 1.19985 \\ \mathrm{C} & 3.50324 & -0.68993 & 1.04545 \\ \mathrm{C} & 1.28240 & -0.12582 & 1.01175 \\ \mathrm{~N} & 1.45087 & -1.47645 & 1.18636 \\ \mathrm{H} & 3.11438 & -2.85936 & 1.32013 \\ \mathrm{H} & 4.56902 & -0.51754 & 1.01621 \\ \mathrm{~N} & 2.57465 & 0.33807 & 0.93232 \\ \mathrm{Ni} & -0.34859 & 0.81639 & 0.85229 \\ \mathrm{C} & -1.94708 & 1.80301 & -0.35659 \\ \mathrm{C} & -2.86560 & 1.03157 & 0.59449 \\ \mathrm{H} & -3.91791 & 1.38317 & 0.57657 \\ \mathrm{H} & -2.85224 & -0.06469 & 0.55573 \\ \mathrm{C} & -1.74350 & 2.91678 & 0.67176 \\ \mathrm{H} & -0.77490 & 3.43392 & 0.69332 \\ \mathrm{H} & -2.55616 & 3.67281 & 0.67001 \\ \mathrm{C} & -1.95370 & 1.72580 & 1.61079 \\ \mathrm{C} & -1.23704 & 1.25649 & 2.70138 \\ \mathrm{H} & -0.61650 & 1.93432 & 3.28421 \\ \mathrm{H} & -1.55415 & 0.35102 & 3.21579\end{array}$




$\begin{array}{lrrr}\mathrm{C} & 0.34285 & -2.41653 & 1.26364 \\ \mathrm{H} & -0.55127 & -1.85593 & 1.54421 \\ \mathrm{H} & 0.17467 & -2.89459 & 0.29401 \\ \mathrm{H} & 0.55480 & -3.17629 & 2.02301 \\ \mathrm{C} & 2.91599 & 1.74039 & 0.76896 \\ \mathrm{H} & 3.49192 & 2.10129 & 1.62864 \\ \mathrm{H} & 3.49986 & 1.89212 & -0.14618 \\ \mathrm{H} & 1.98067 & 2.30127 & 0.70251 \\ \mathrm{C} & -0.57965 & 0.98858 & -1.34698 \\ \mathrm{H} & 0.35317 & 1.53894 & -1.45695 \\ \mathrm{C} & -1.79193 & 1.78614 & -1.81945 \\ \mathrm{H} & -2.53825 & 1.22802 & -2.38777 \\ \mathrm{H} & -1.56351 & 2.74437 & -2.29120 \\ \mathrm{C} & -0.47996 & -0.44634 & -1.70354 \\ \mathrm{C} & -1.60340 & -1.28716 & -1.83021 \\ \mathrm{C} & 0.78497 & -1.00933 & -1.97854 \\ \mathrm{C} & -1.46662 & -2.62753 & -2.20069 \\ \mathrm{H} & -2.59642 & -0.89659 & -1.62798 \\ \mathrm{C} & 0.92163 & -2.34210 & -2.35557 \\ \mathrm{H} & 1.67064 & -0.38708 & -1.87417 \\ \mathrm{C} & -0.20561 & -3.16822 & -2.46643 \\ \mathrm{H} & -2.35423 & -3.25073 & -2.28452 \\ \mathrm{H} & 1.91270 & -2.74026 & -2.56147 \\ \mathrm{H} & -0.10103 & -4.20931 & -2.76055\end{array}$

(b) (B3LYP/6-31++G**)

$\mathbf{I}^{\mathbf{B}}$

SCF Energy: -2317.04267626 hartrees

$\begin{array}{lrrr}\mathrm{C} & 0.35767 & -3.21498 & -1.29844 \\ \mathrm{C} & -0.31903 & -3.71206 & -0.23632 \\ \mathrm{C} & -0.42553 & -1.42969 & -0.10509 \\ \mathrm{~N} & 0.27948 & -1.83282 & -1.20859 \\ \mathrm{H} & 0.88186 & -3.71821 & -2.09341 \\ \mathrm{H} & -0.50185 & -4.72945 & 0.06613 \\ \mathrm{~N} & -0.78861 & -2.61808 & 0.47831 \\ \mathrm{Ni} & -0.71268 & 0.37831 & 0.53350 \\ \mathrm{C} & 0.94201 & -0.91656 & -2.12830 \\ \mathrm{H} & 2.02558 & -0.95540 & -1.99784 \\ \mathrm{H} & 0.60189 & 0.09168 & -1.90241 \\ \mathrm{H} & 0.68571 & -1.16978 & -3.15997 \\ \mathrm{C} & -1.55531 & -2.71438 & 1.71032 \\ \mathrm{H} & -1.03432 & -3.34646 & 2.43354 \\ \mathrm{H} & -2.54916 & -3.13097 & 1.52308 \\ \mathrm{H} & -1.65333 & -1.70953 & 2.11967 \\ \mathrm{C} & -2.26799 & 1.11190 & -0.56251 \\ \mathrm{C} & -3.47439 & 0.72839 & 0.38390 \\ \mathrm{H} & -3.73100 & -0.32317 & 0.43249 \\ \mathrm{H} & -3.54463 & 1.27324 & 1.31754 \\ \mathrm{C} & -2.76121 & 2.55630 & -0.74405 \\ \mathrm{H} & -2.45846 & 3.06726 & -1.65185 \\ \mathrm{H} & -2.79423 & 3.17500 & 0.14524 \\ \mathrm{C} & -2.98577 & 0.59529 & -1.82030\end{array}$




$\begin{array}{lrrr}\mathrm{H} & -3.21461 & -0.46437 & -1.85284 \\ \mathrm{H} & -2.69284 & 1.03370 & -2.76853 \\ \mathrm{C} & -3.79369 & 1.46049 & -0.87998 \\ \mathrm{C} & 0.83886 & 0.97113 & 1.63488 \\ \mathrm{H} & 0.89993 & 0.33271 & 2.51510 \\ \mathrm{C} & -0.18946 & 1.95222 & 1.60342 \\ \mathrm{H} & -0.06166 & 2.86689 & 1.02821 \\ \mathrm{H} & -0.80068 & 2.08271 & 2.49569 \\ \mathrm{C} & 2.09415 & 1.01362 & 0.86003 \\ \mathrm{C} & 2.38640 & 2.01510 & -0.08601 \\ \mathrm{C} & 3.06730 & 0.01345 & 1.05279 \\ \mathrm{C} & 3.57543 & 2.00379 & -0.80972 \\ \mathrm{H} & 1.67401 & 2.81415 & -0.25493 \\ \mathrm{C} & 4.25555 & 0.00002 & 0.32963 \\ \mathrm{H} & 2.87676 & -0.76661 & 1.78380 \\ \mathrm{C} & 4.51942 & 0.99442 & -0.61431 \\ \mathrm{H} & 3.77041 & 2.79384 & -1.52812 \\ \mathrm{H} & 4.98309 & -0.78557 & 0.50765 \\ \mathrm{H} & 5.44692 & 0.99018 & -1.17584\end{array}$

\section{$\mathbf{I}^{\mathbf{F}}$}

SCF Energy: -2317.04342991 hartrees

$\begin{array}{lrrr}\mathrm{C} & 0.61621 & -2.29580 & 2.03331 \\ \mathrm{C} & -0.44317 & -1.79483 & 2.71089 \\ \mathrm{C} & -0.29279 & -0.64903 & 0.74406 \\ \mathrm{~N} & 0.69565 & -1.58759 & 0.84249 \\ \mathrm{H} & 1.30629 & -3.08164 & 2.29017 \\ \mathrm{H} & -0.85038 & -2.05765 & 3.67256 \\ \mathrm{~N} & -0.98496 & -0.79523 & 1.91345 \\ \mathrm{Ni} & -0.68532 & 0.48430 & -0.79519 \\ \mathrm{C} & -2.26782 & 0.02633 & -2.78308 \\ \mathrm{C} & -1.20150 & 1.26902 & -2.52522 \\ \mathrm{C} & -1.10086 & 0.27468 & -3.68302 \\ \mathrm{C} & -2.70666 & 1.45584 & -2.69809 \\ \mathrm{C} & -2.05347 & -0.73446 & -1.54714 \\ \mathrm{H} & -1.64014 & -1.73529 & -1.68791 \\ \mathrm{H} & -2.91254 & -0.76584 & -0.87279 \\ \mathrm{H} & -1.34065 & 0.65974 & -4.67563 \\ \mathrm{H} & -0.27638 & -0.43372 & -3.68245 \\ \mathrm{H} & -3.04374 & 1.89770 & -3.63749 \\ \mathrm{H} & -3.28862 & 1.77744 & -1.83751 \\ \mathrm{C} & -0.09770 & 2.58796 & -0.08226 \\ \mathrm{C} & 1.14947 & 2.05218 & -0.03017 \\ \mathrm{H} & -0.42364 & 3.21244 & -0.90085 \\ \mathrm{H} & -0.72912 & 2.58007 & 0.79874 \\ \mathrm{H} & 1.45271 & 1.55930 & 0.88889 \\ \mathrm{C} & 2.19950 & 2.14473 & -1.04932 \\ \mathrm{C} & 3.50873 & 1.75584 & -0.71426 \\ \mathrm{C} & 1.96050 & 2.61203 & -2.35450 \\ \mathrm{C} & 4.54648 & 1.84480 & -1.63715 \\ \mathrm{H} & 3.7143 & 1.39236 & 0.28883 \\ \mathrm{C} & 2.99762 & 2.69361 & -3.27703 \\ \mathrm{H} & 0.95263 & 2.87356 & -2.65091 \\ \mathrm{C} & 4.29496 & 2.31625 & -2.92516\end{array}$




$\begin{array}{rrrr}\mathrm{H} & 5.55017 & 1.54855 & -1.35190 \\ \mathrm{H} & 2.79224 & 3.05006 & -4.28060 \\ \mathrm{H} & 5.09991 & 2.38706 & -3.64817 \\ \mathrm{C} & 1.68446 & -1.84404 & -0.19811 \\ \mathrm{H} & 1.54612 & -2.84324 & -0.61791 \\ \mathrm{H} & 1.55032 & -1.10316 & -0.98392 \\ \mathrm{H} & 2.69438 & -1.75839 & 0.20828 \\ \mathrm{C} & -2.15120 & -0.00248 & 2.27910 \\ \mathrm{H} & -2.42732 & 0.60552 & 1.41999 \\ \mathrm{H} & -2.98593 & -0.65735 & 2.53805 \\ \mathrm{H} & -1.92650 & 0.64749 & 3.12857\end{array}$

[1.1.1]-propellane, 1

SCF Energy: -194.05881647 hartrees

$\begin{array}{lrrr}\mathrm{C} & 1.69244 & 1.00011 & 1.86117 \\ \mathrm{H} & 1.86972 & 0.08528 & 2.41619 \\ \mathrm{H} & 1.86958 & 1.91508 & 2.41602 \\ \mathrm{C} & 0.54587 & 0.99993 & 0.86342 \\ \mathrm{C} & 2.04725 & 1.00000 & 0.38319 \\ \mathrm{C} & 1.09861 & 2.12559 & 0.00405 \\ \mathrm{H} & 0.76860 & 2.17251 & -1.02806 \\ \mathrm{H} & 1.25114 & 3.08764 & 0.48115 \\ \mathrm{C} & 1.09878 & -0.12580 & 0.00426 \\ \mathrm{H} & 1.25147 & -1.08774 & 0.48154 \\ \mathrm{H} & 0.76879 & -0.17297 & -1.02784\end{array}$

[2.1.1]-propellane

SCF Energy: -233.38270555 hartrees

$\begin{array}{llrc}\mathrm{C} & 1.38901 & 1.12550 & 2.22345 \\ \mathrm{C} & 0.67673 & 1.01882 & 0.84144 \\ \mathrm{C} & 2.16685 & 0.91588 & 0.14475 \\ \mathrm{C} & 1.20680 & 2.04697 & -0.12677 \\ \mathrm{H} & 0.74731 & 2.05626 & -1.11093 \\ \mathrm{H} & 1.45291 & 3.03409 & 0.25338 \\ \mathrm{C} & 1.14735 & -0.19379 & 0.07797 \\ \mathrm{H} & 1.34296 & -1.10732 & 0.63180 \\ \mathrm{H} & 0.68340 & -0.35721 & -0.89050 \\ \mathrm{C} & 2.78308 & 1.02941 & 1.57166 \\ \mathrm{H} & 3.37036 & 0.15128 & 1.84267 \\ \mathrm{H} & 3.41684 & 1.90994 & 1.68298 \\ \mathrm{H} & 1.17101 & 2.06451 & 2.73347 \\ \mathrm{H} & 1.12445 & 0.30588 & 2.89253\end{array}$


Abbreviated (annotated) ORCA output file for flipspin broken symmetry calculation on $\mathrm{I}^{\mathrm{B}}$

(triplet, before flipspin)

* MULLIKEN POPULATION ANALYSiS *

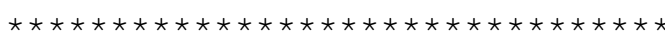

\begin{tabular}{|c|c|c|c|c|}
\hline \multicolumn{5}{|c|}{ MULLIKEN ATOMIC CHARGES AND SPIN POPULATIONS } \\
\hline 0 & $\mathrm{Ni}$ & $\therefore \quad-1.169986$ & 1.358110 & --- \\
\hline 32 & $\mathrm{C}$ & 0.144962 & -0.610711 & (coordinated bridgehead carbon) \\
\hline 33 & $\mathrm{C}$ & -0.343900 & 0.086987 & \\
\hline 34 & $\mathrm{H}$ & 0.189770 & -0.002723 & \\
\hline 35 & $\mathrm{H}$ & 0.195152 & -0.002788 & \\
\hline 36 & $\mathrm{C}$ & -0.424376 & 0.012857 & \\
\hline 37 & $\mathrm{H}$ & 0.201012 & -0.001812 & \\
\hline 38 & $\mathrm{H}$ & 0.198657 & -0.001944 & \\
\hline 39 & $\mathrm{C}$ & -0.334349 & 0.012276 & \\
\hline 40 & $\mathrm{H}$ & 0.193592 & -0.002121 & \\
\hline 41 & $\mathrm{H}$ & 0.193408 & -0.002111 & \\
\hline 42 & $\mathrm{C}$ & 0.190804 & 0.692992 & (non-coordinated bridgehead carbon) \\
\hline
\end{tabular}

Sum of atomic charges : $\quad-0.0000000$

Sum of atomic spin populations: 2.0000000

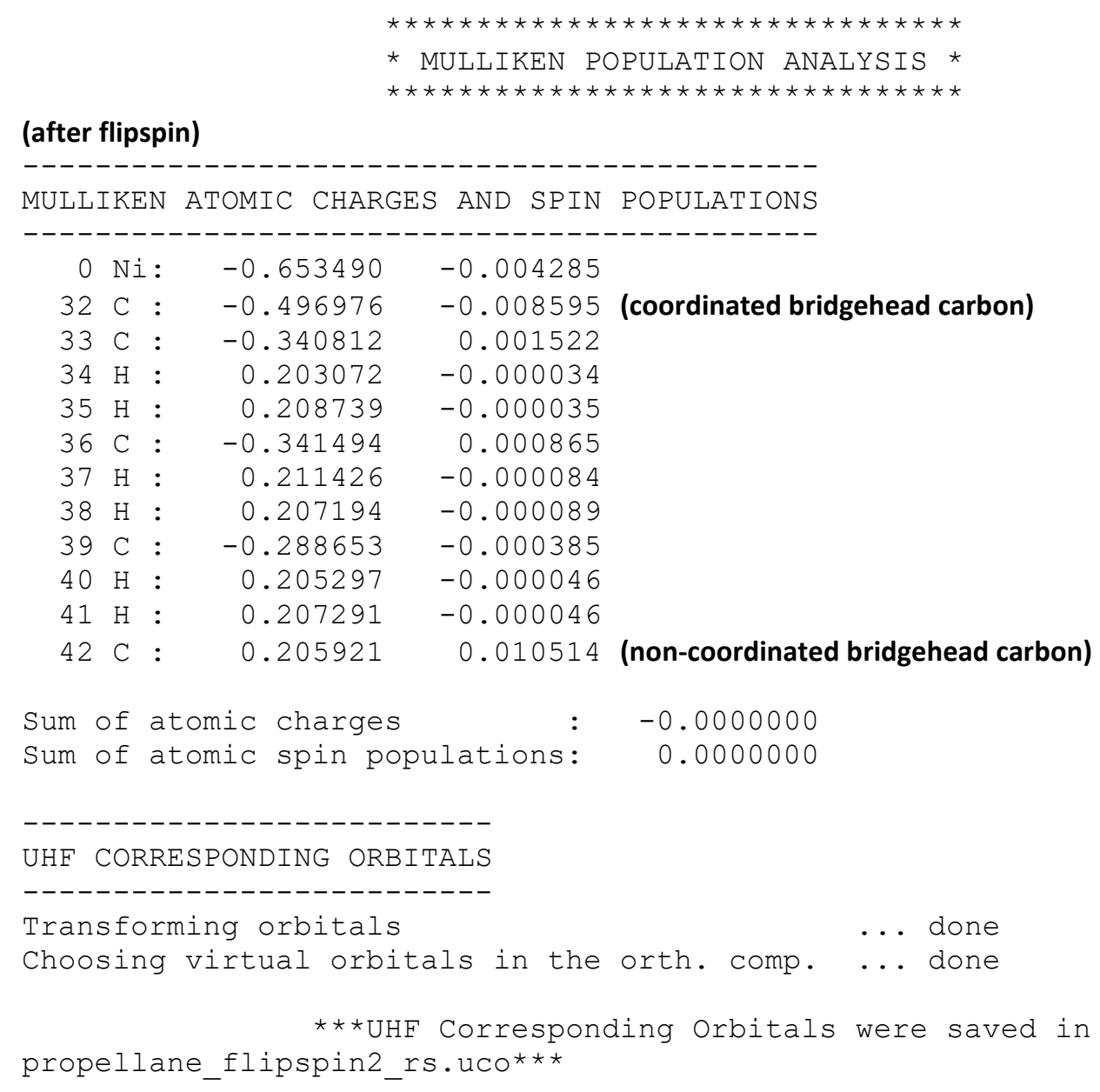


Now the overlap between the corresponding orbitals will be plotted Since there are an equal number of alpha and beta orbitals each orbital in the alpha set is matched by an orbital in the beta set. If all overlaps are close to one you have a normal UHF solution with some spin polarization If you have a broken symmetry solution there will be overlaps that are significantly smaller than one. These orbitals are the non-orthogonal magneticorbitals of the broken symmetry UHF solution.

\begin{tabular}{|c|c|}
\hline Orbital & Overlap (*) \\
\hline-------- & ----------- \\
\hline $0:$ & 1.00000 \\
\hline $1:$ & 1.00000 \\
\hline $2:$ & 1.00000 \\
\hline $3:$ & 1.00000 \\
\hline $4:$ & 1.00000 \\
\hline $5:$ & 1.00000 \\
\hline $6:$ & 1.00000 \\
\hline $7:$ & 1.00000 \\
\hline $8:$ & 1.00000 \\
\hline 9: & 1.00000 \\
\hline 10: & 1.00000 \\
\hline 11: & 1.00000 \\
\hline 12: & 1.00000 \\
\hline 13: & 1.00000 \\
\hline 14: & 1.00000 \\
\hline 15: & 1.00000 \\
\hline 16: & 1.00000 \\
\hline 17: & 1.00000 \\
\hline 18: & 1.00000 \\
\hline 19: & 1.00000 \\
\hline $20:$ & 1.00000 \\
\hline 21: & 1.00000 \\
\hline $22:$ & 1.00000 \\
\hline $23:$ & 1.00000 \\
\hline $24:$ & 1.00000 \\
\hline $25:$ & 1.00000 \\
\hline 26: & 1.00000 \\
\hline 27: & 1.00000 \\
\hline $28:$ & 1.00000 \\
\hline $29:$ & 1.00000 \\
\hline $30:$ & 1.00000 \\
\hline $31:$ & 1.00000 \\
\hline $32:$ & 1.00000 \\
\hline $33:$ & 1.00000 \\
\hline $34:$ & 1.00000 \\
\hline $35:$ & 1.00000 \\
\hline $36:$ & 1.00000 \\
\hline $37:$ & 1.00000 \\
\hline $38:$ & 1.00000 \\
\hline 39: & 1.00000 \\
\hline $40:$ & 1.00000 \\
\hline 41: & 1.00000 \\
\hline $42:$ & 1.00000 \\
\hline $43:$ & 1.00000 \\
\hline $44:$ & 1.00000 \\
\hline $45:$ & 1.00000 \\
\hline 46: & 1.00000 \\
\hline $47:$ & 1.00000 \\
\hline 48: & 1.00000 \\
\hline
\end{tabular}




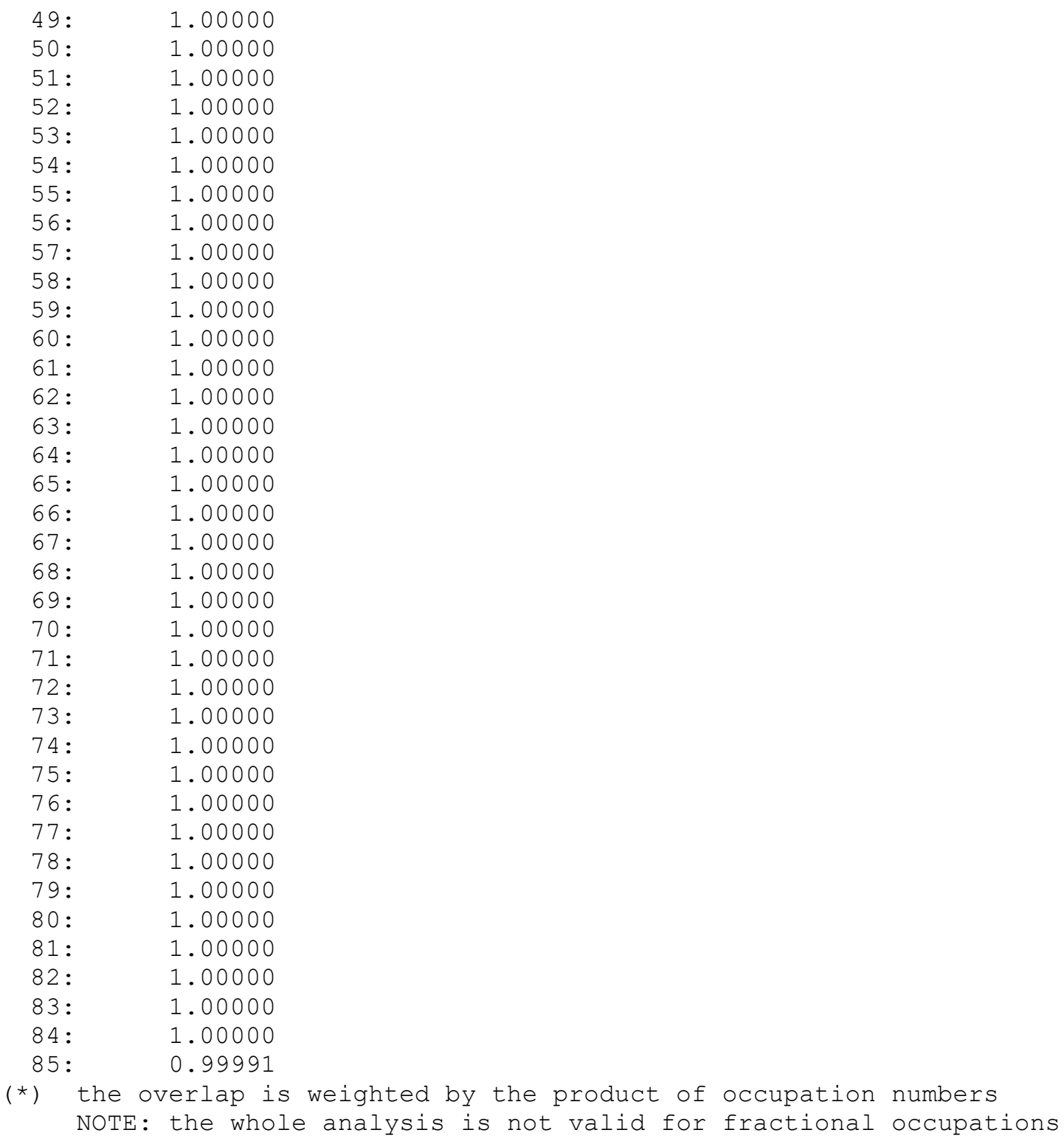

BROKEN SYMMETRY MAGNETIC COUPLING ANALYSIS

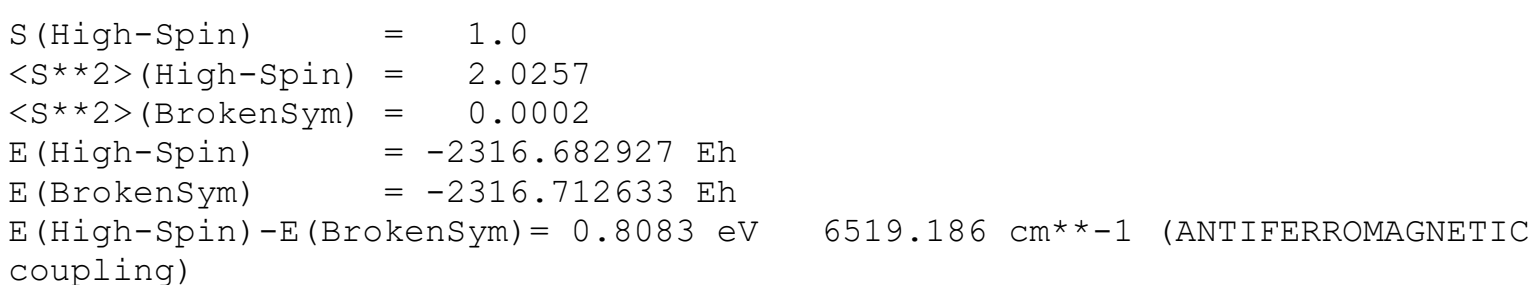

| Spin-Hamiltonian Analysis based on $\mathrm{H}(\mathrm{HDVV})=-2 \mathrm{~J} * \mathrm{SA} * \mathrm{SB}$ |

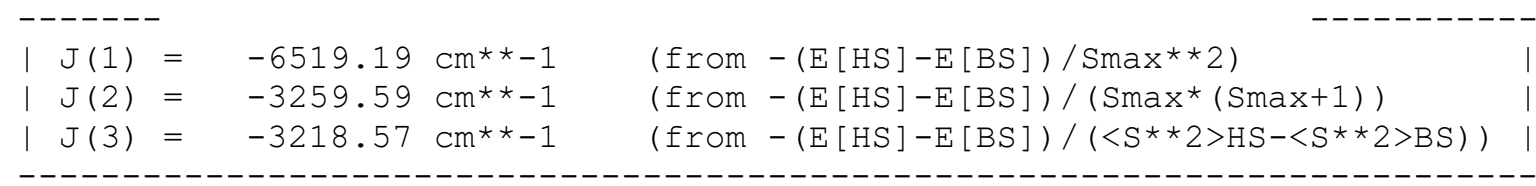




\section{References}

1) Kurandina, D.; Parasram, M.; Gevorgyan, V., Visible Light-Induced Room-Temperature Heck Reaction of Functionalized Alkyl Halides with Vinyl Arenes/Heteroarenes, Angew. Chem. Int. Ed. 2017, 56, 1421214216.

2) Wei, B.; Chen, C.; You, C.; Lv, H.; Zhang, W., Efficient synthesis of $(S, R)$-Bn-Yanphos and Rh/( $S, R)-\mathrm{Bn}$ Yanphos catalyzed asymmetric hydroformylation of vinyl heteroarenes, Org. Chem. Front. 2017, 4, 288291.

3) Aukland, M. H.; Talbot, F. J. T.; Fernández-Salas, J. A.; Ball, M.; Pulis, A. P.; Procter, D. J., An Interrupted Pummerer/Nickel-Catalysed Cross-Coupling Sequence, Angew. Chem. Int. Ed. 2018, 57, 9785-9789.

4) Maza, R. J.; Davenport, E.; Miralles, N.; Carbó, J. J.; Fernández, E., Transition-Metal-Free Allylic Borylation of 1,3-Dienes, Org. Lett. 2019, 21, 2251-2255.

5) Frisch, M. J.; Trucks, G. W.; Schlegel, H. B.; Scuseria, G. E.; Robb, M. A.; Cheeseman, J. R.; Scalmani, G.; Barone, V.; Mennucci, B.; Petersson, G. A.; Nakatsuji, H.; Caricato, M.; Li, X.; Hratchian, H. P.; Izmaylov, A. F.; Bloino, J.; Zheng, G.; Sonnenberg, J. L.; Hada, M.; Ehara, M.; Toyota, K.; Fukuda, R.; Hasegawa, J.; Ishida, M.; Nakajima, T.; Honda, Y.; Kitao, O.; Nakai, H.; Vreven, T.; Montgomery, Jr. J. A.; Peralta, J. E.; Ogliaro, F.; Bearpark, M.; Heyd, J. J.; Brothers, E.; Kudin, K. N.; Staroverov, V. N.; Keith, T.; Kobayashi, R.; Normand, J.; Raghavachari, K.; Rendell, A.; Burant, J. C.; Iyengar, S. S.; Tomasi, J.; Cossi, M.; Rega, N.; Millam, J. M.; Klene, M.; Knox, J. E.; Cross, J. B.; Bakken, V.; Adamo, C.; Jaramillo, J.; Gomperts, R.; Stratmann, R. E.; Yazyev, O.; Austin, A. J.; Cammi, R.; Pomelli, C.; Ochterski, J. W.; Martin, R. L.; Morokuma, K.; Zakrzewski, V. G.; Voth, G. A.; Salvador, P.; Dannenberg, J. J.; Dapprich, S.; Daniels, A. D.; Farkas, O.; Foresman, J. B.; Ortiz, J. V.; Cioslowski J.; Fox, D. J. Gaussian 09, Revision D.01, Gaussian, Inc., Wallingford CT (2013).

6) (a) Becke, A. D., Density-functional thermochemistry. III. The role of exact exchange, J. Chem. Phys. 1993, 98, 5648-5652; (b) Lee, C.; Yang, W.; Parr, R. G., Development of the Colle-Salvetti correlation-energy formula into a functional of the electron density, Phys. Rev. B 1988, 37, 785-789; (c) Vosko, S. H.; Wilk, L.; Nusair, M., Accurate spin-dependent electron liquid correlation energies for local spin density calculations: a critical analysis, Can. J. Phys. 1980, 58, 1200-1211; (d) Stephens, P. J.; Devlin, F. J.; Chabalowski, C. F.; Frisch, M. J., Ab Initio Calculation of Vibrational Absorption and Circular Dichroism Spectra Using Density Functional Force Fields, J. Phys. Chem. 1994, 98, 11623-11627.

7) Grimme, S.; Antony, J.; Ehrlich, S.; Krieg, H., A consistent and accurate ab initio parametrization of density functional dispersion correction (DFT-D) for the 94 elements H-Pu, J. Chem. Phys. 2010, 132, 154104.

8) (a) Hariharan, P. C.; Pople, J. A., The influence of polarization functions on molecular orbital hydrogenation energies, Theor. Chim. Acta 1973, 28, 213-222; (b) Frisch, M. J., Pople, J. A.; Binkley, J. S., Self-consistent molecular orbital methods 25. Supplementary functions for Gaussian basis sets, J. Chem. Phys. 1984, 80, 3265-3269.

9) Marenich, A. V.; Cramer, C. J.; Truhlar, D. G., Universal Solvation Model Based on Solute Electron Density and on a Continuum Model of the Solvent Defined by the Bulk Dielectric Constant and Atomic Surface Tensions, J. Phys. Chem. B 2009, 113, 6378-6396.

10) Neese, F., The ORCA program system, Wiley Interdiscip. Rev. Comput. Mol. Sci. 2012, 2, 73-78.

11) Grimme, S.; Ehrlich, S.; L. Goerigk, Effect of the damping function in dispersion corrected density functional theory, J. Comput. Chem. 2011, 32, 1456-1465.

12) Neese, F.; Wennmohs, F.; Hansen, A.; Becker U., Efficient, approximate and parallel Hartree-Fock and hybrid DFT calculations. A 'chain-of-spheres' algorithm for the Hartree-Fock exchange, Chem. Phys. 2009, 356, 98-109.

13) Weigend, F., Accurate Coulomb-fitting basis sets for $\mathrm{H}$ to Rn, Phys. Chem. Chem. Phys. 2006, 8, 10571065.

14) Neese, F., Definition of corresponding orbitals and the diradical character in broken symmetry DFT calculations on spin coupled systems, J. Phys. Chem. Solids 2004, 65, 781-785.

15) Della, E. W.; Pigou, P. E.; Schiesser, C. H.; Taylor, D. K., Experimental and Calculated Activation Parameters for Ring Opening of the 1-Bicyclo[1.1.1]pentyl Radical: The Effect of Bridgehead Substituents, J. Org. Chem. 1991, 56, 4659-4664.

16) (a) Biegler-König, F.W; Bader, R.F.W.; T.H. Tang, Calculation of the Average Properties of Atoms in Molecules. II, J. Comput. Chem. 1982, 13, 317-328; (b) AIMAll (Version 15.09.27), Todd A. Keith, TK Gristmill Software, Overland Park KS, USA, 2015 (aim.tkgristmill.com) 


\section{NMR Spectra}

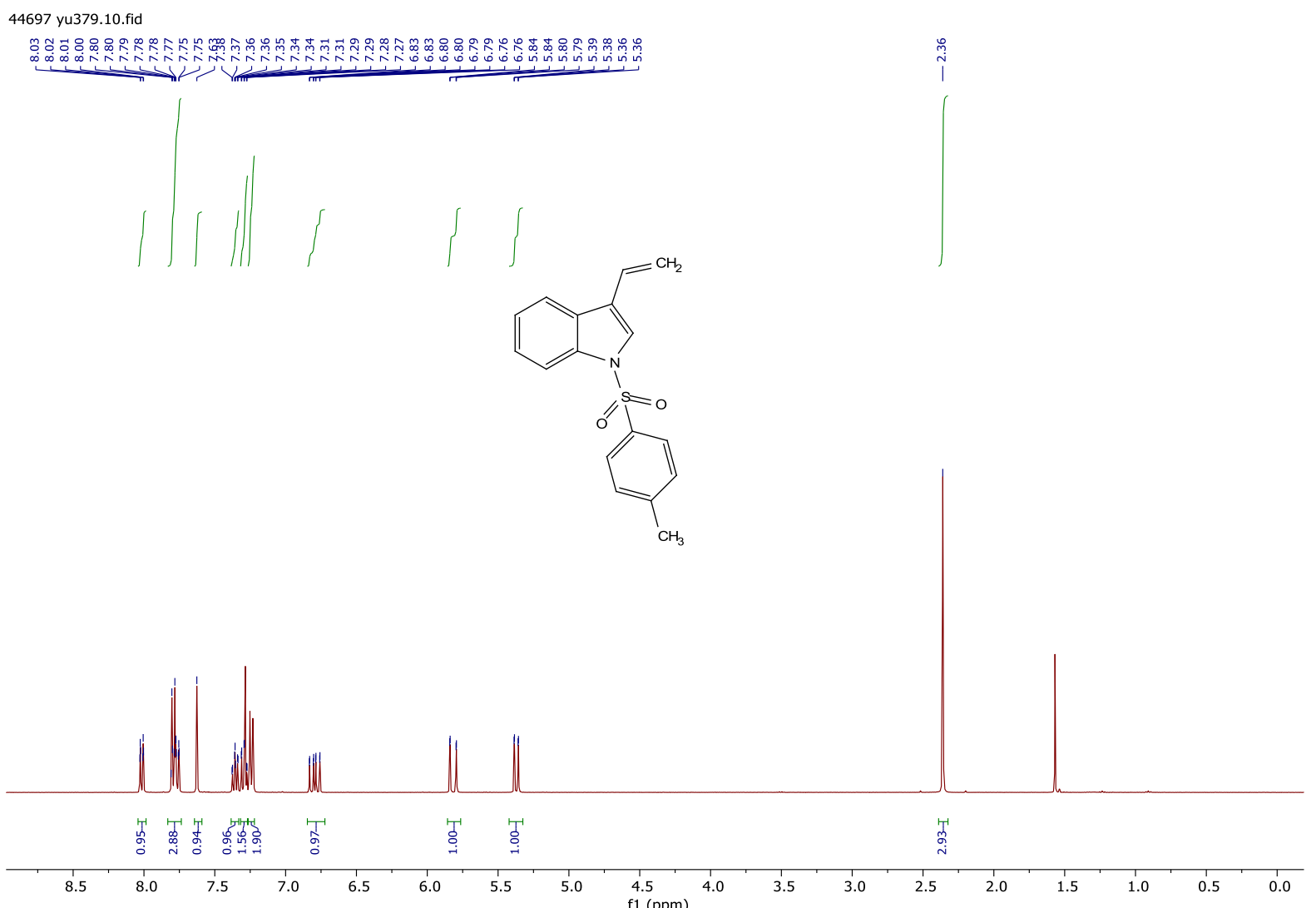

44697 yu379.11.fid
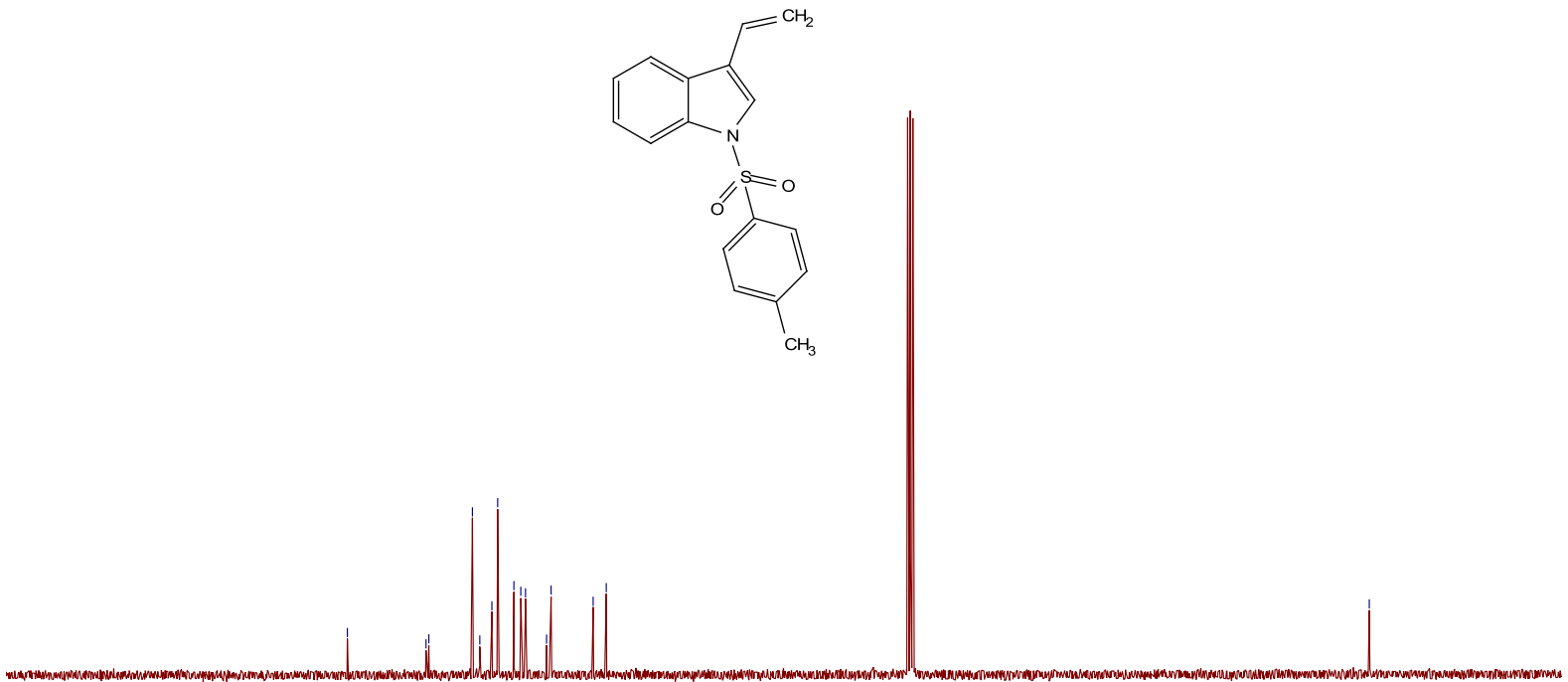

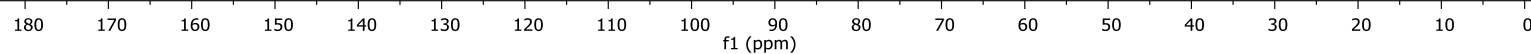




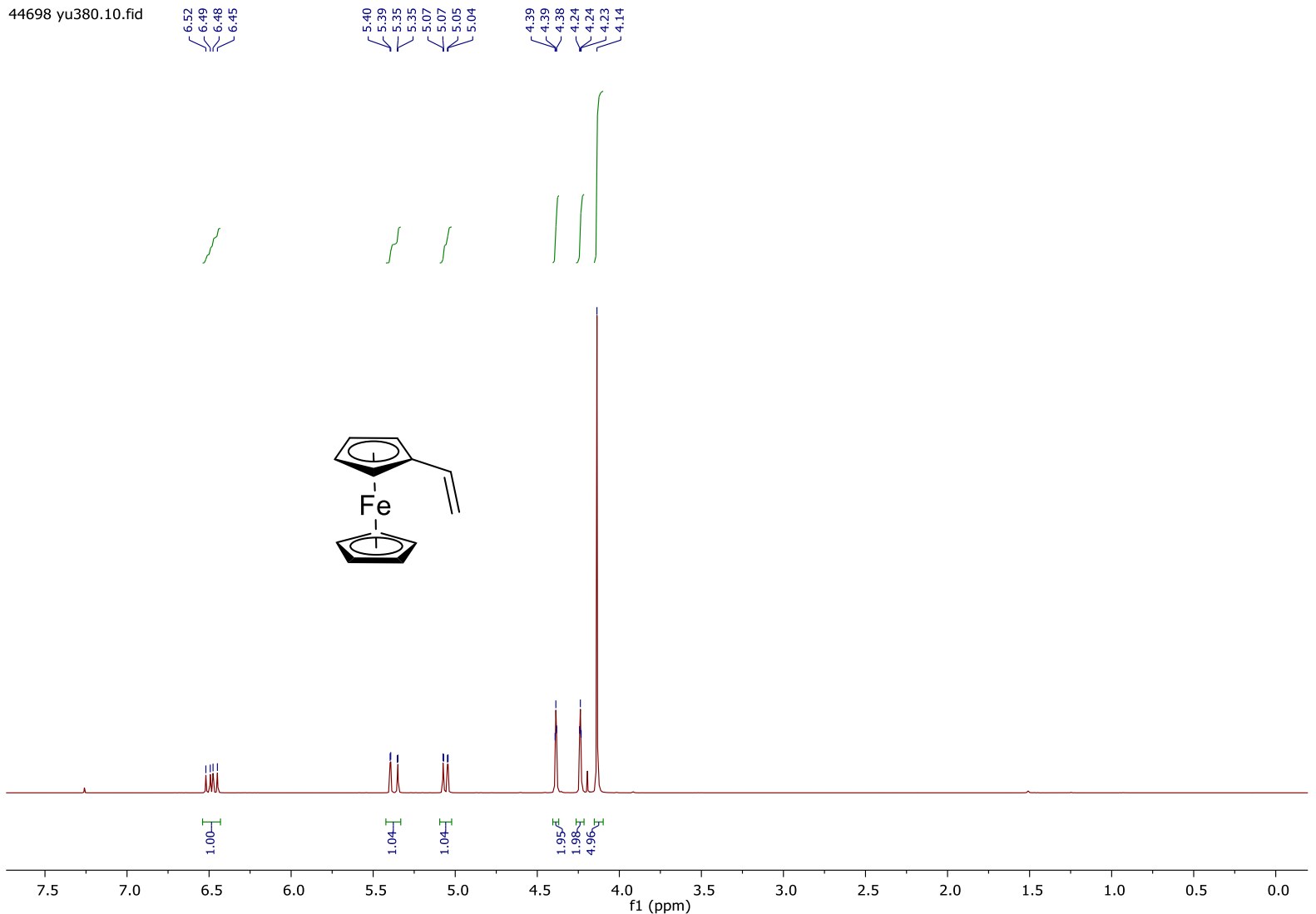

44698 yu380.11.fid

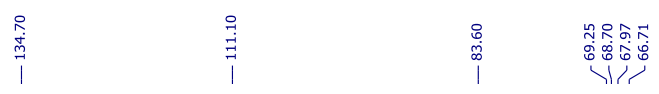

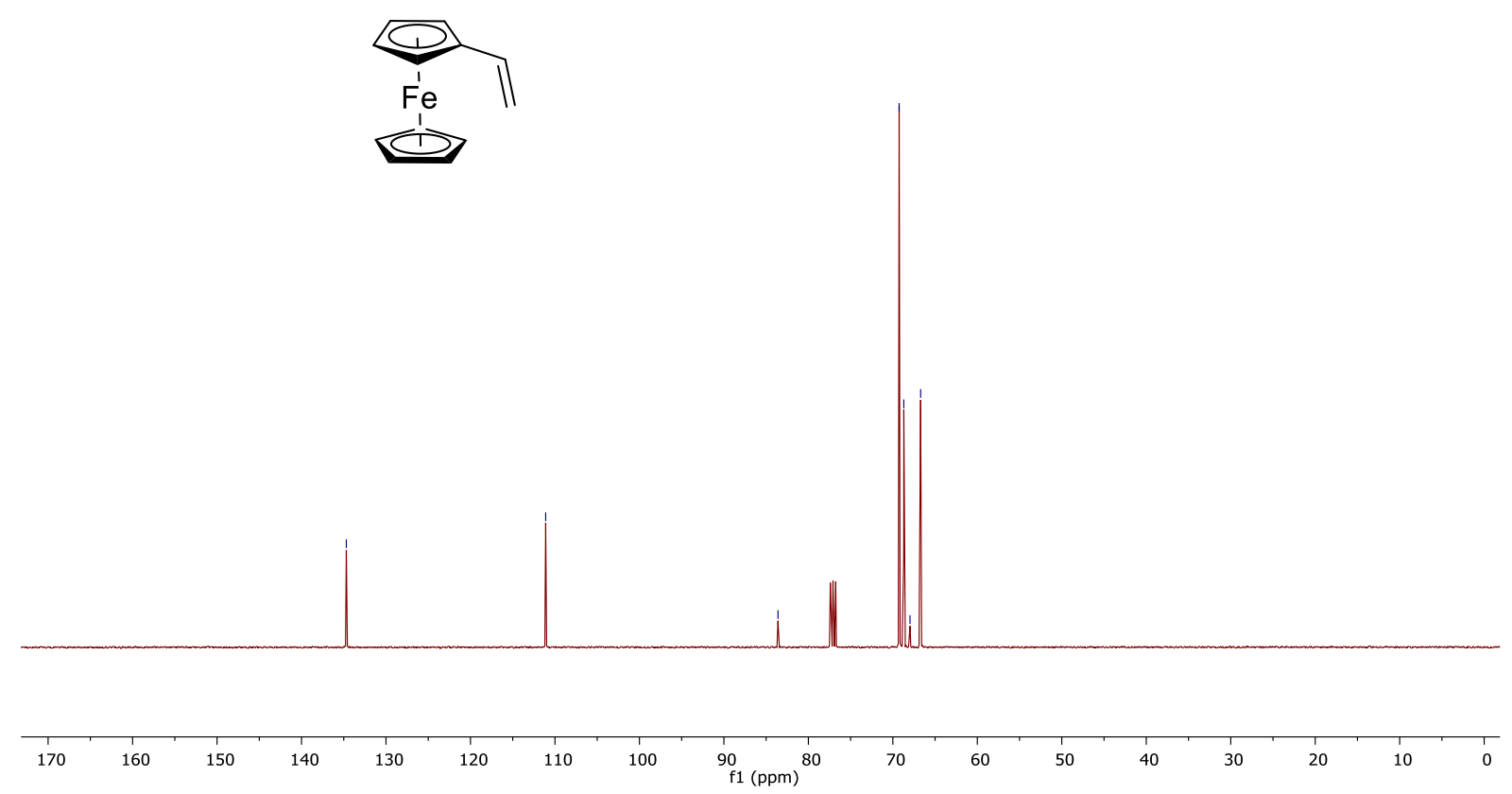




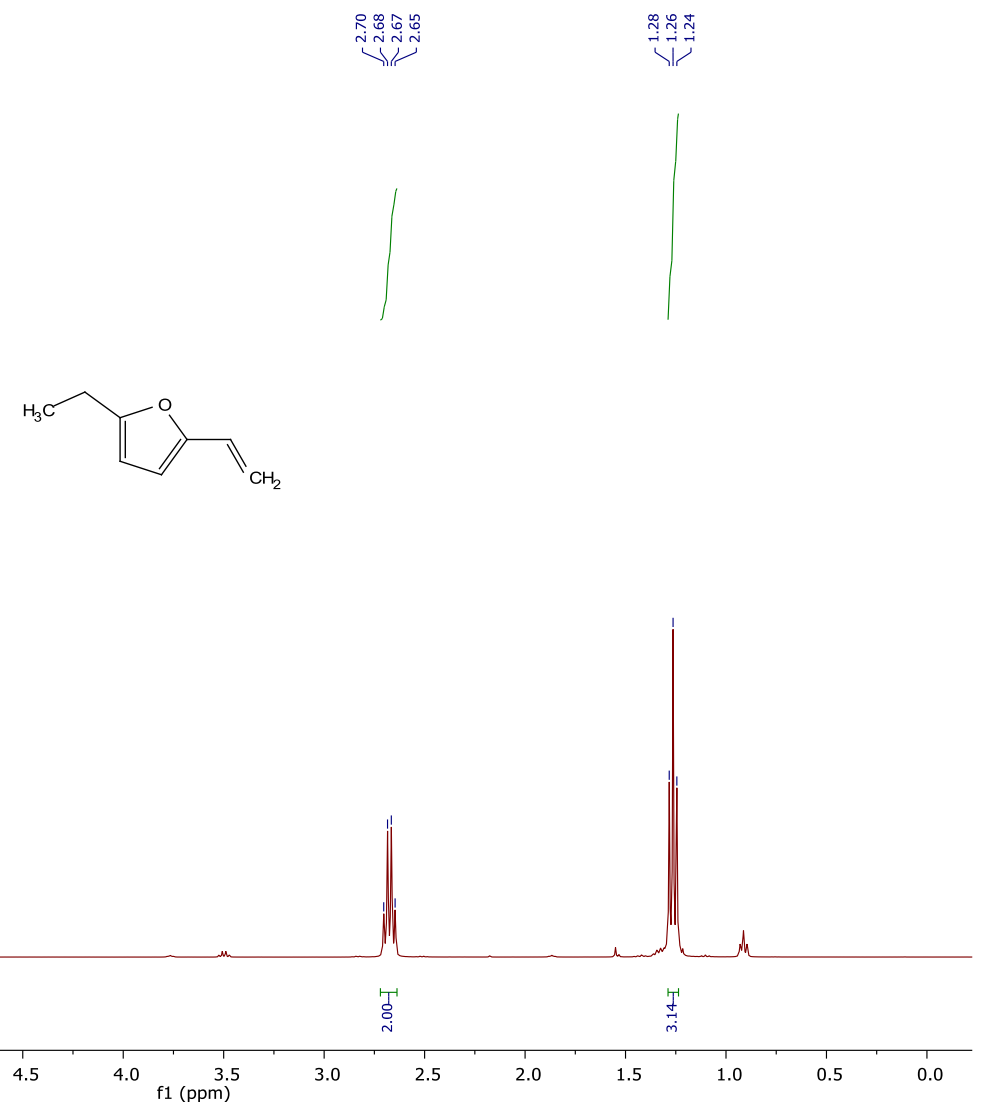

va/dn18877 yu342

single pulse decoupled gated NOE

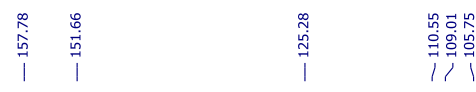
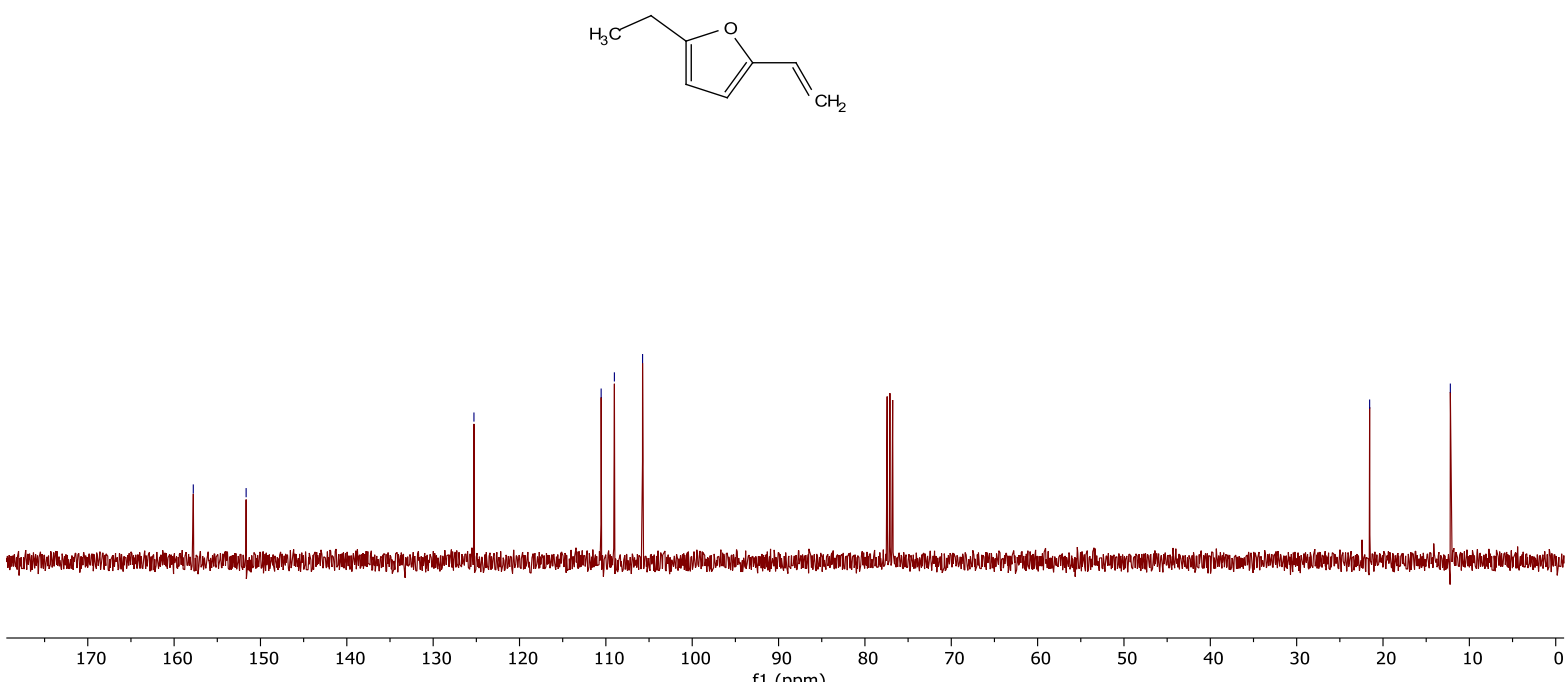


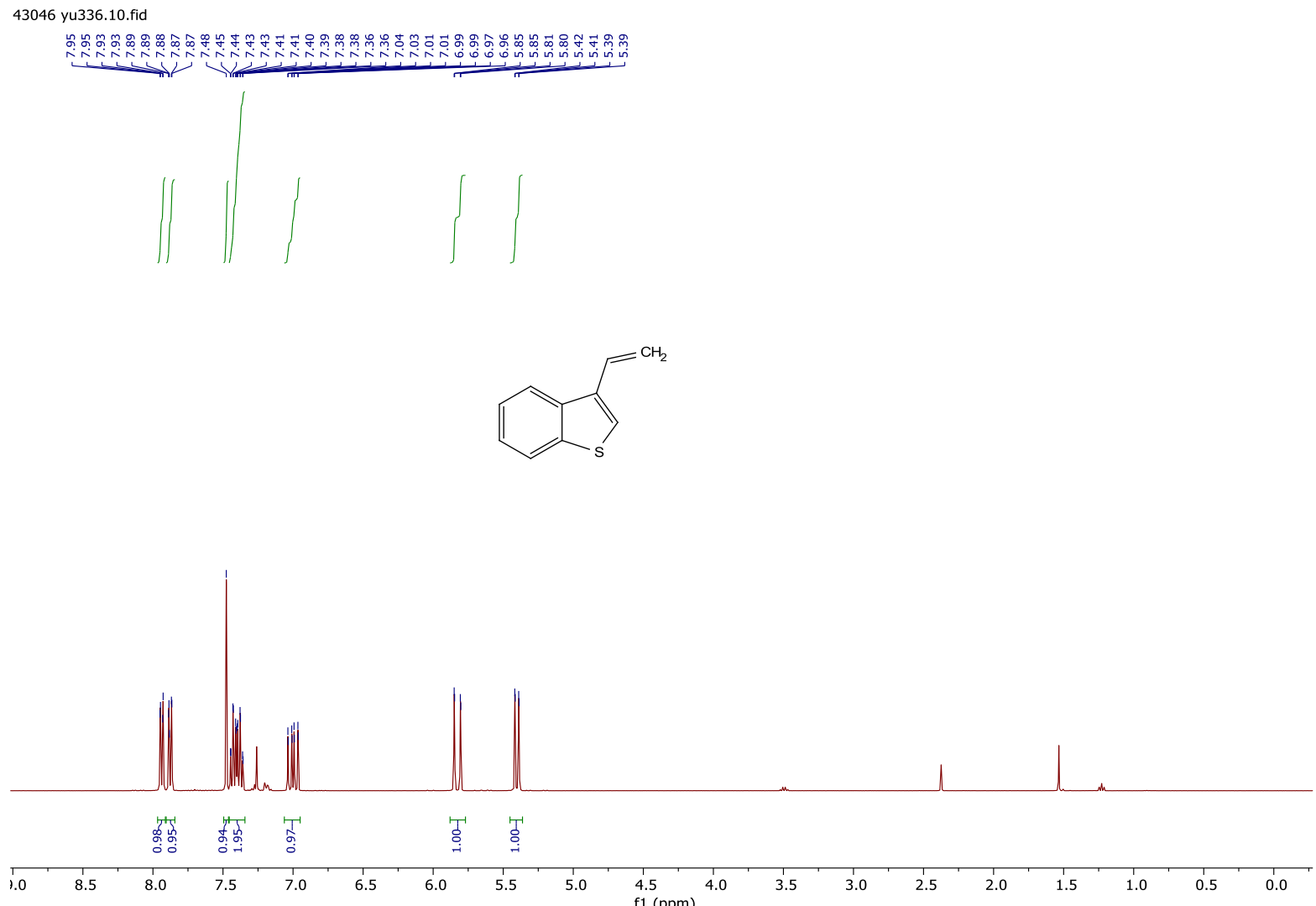

43046 yu336.11.fid

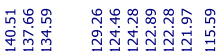

11 | ता ।
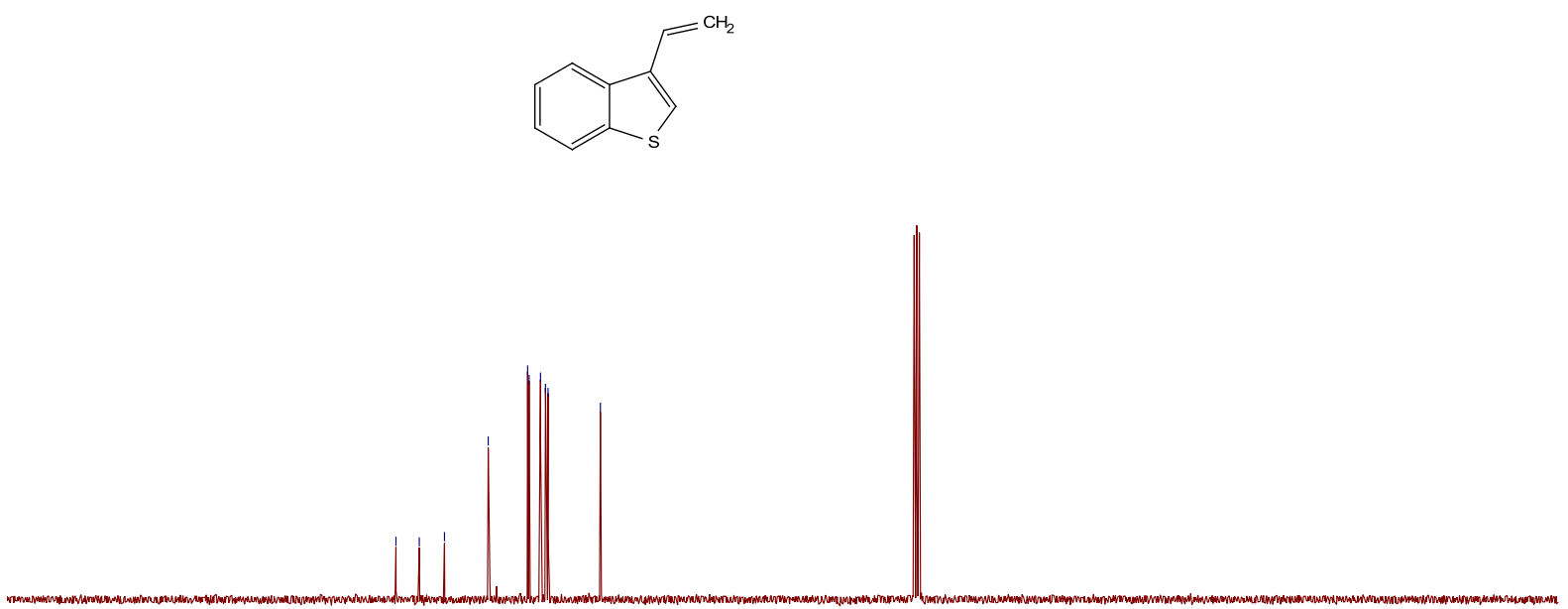

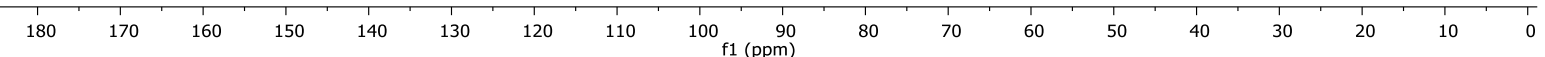


42471 yu324.10.fid
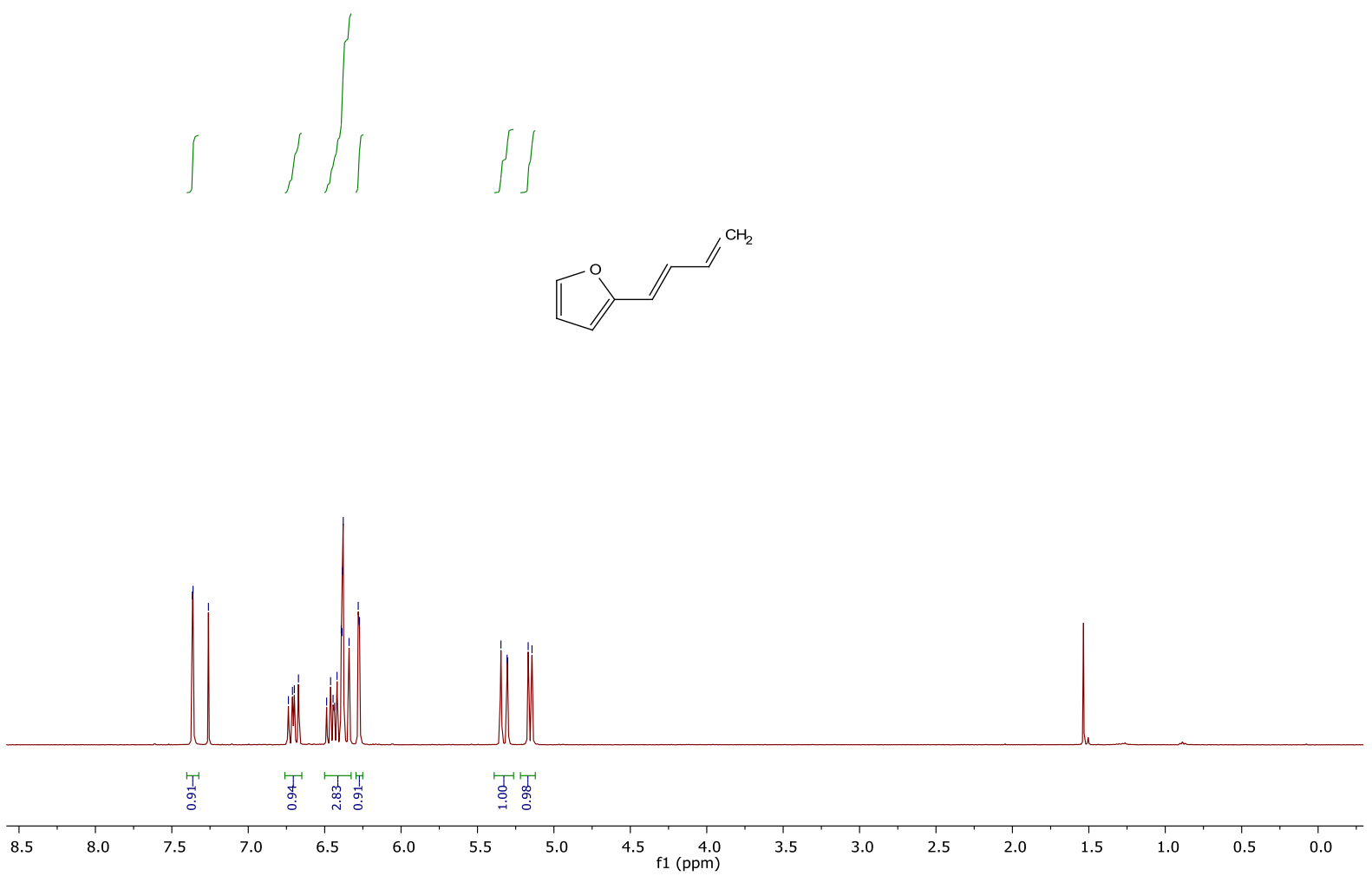

42471 yu324.11.fid

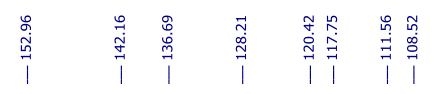
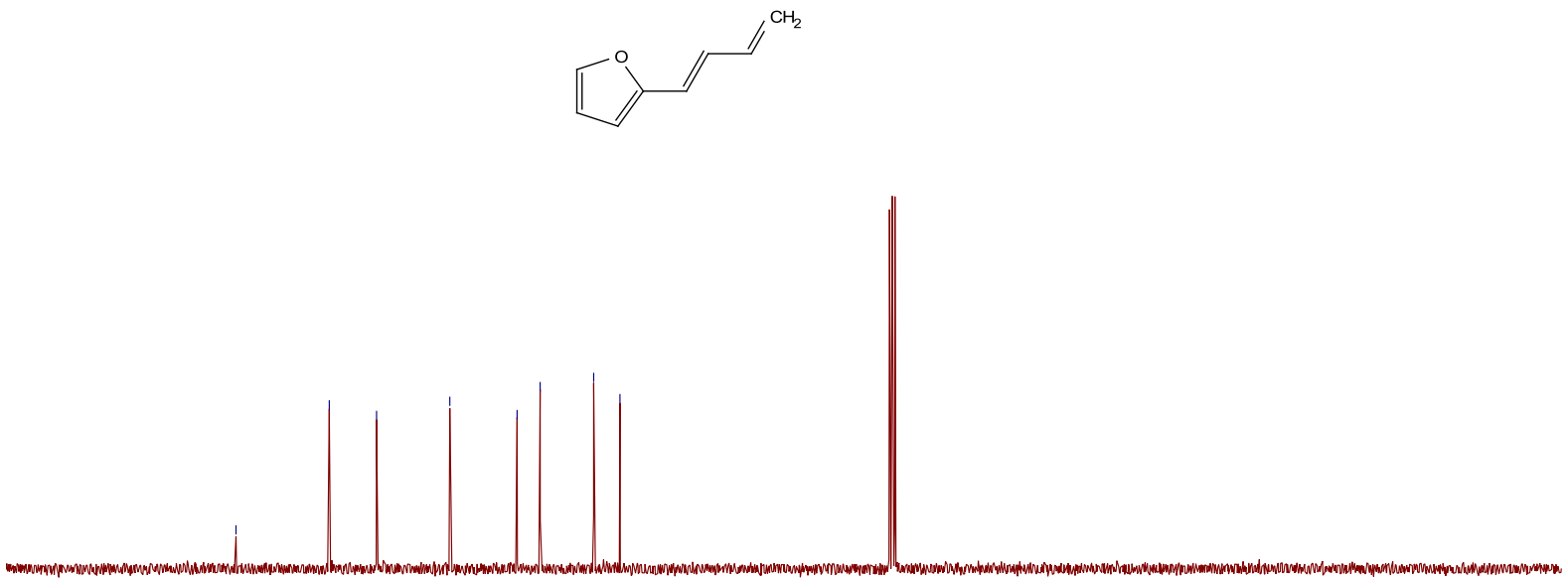

\begin{tabular}{|c|c|c|c|c|c|c|c|c|c|c|c|c|c|c|c|}
\hline 170 & 160 & 150 & 140 & 130 & 120 & 110 & 100 & $\begin{array}{c}90 \\
\mathrm{f} 1(\mathrm{ppm})\end{array}$ & 80 & 70 & 60 & 50 & 40 & 30 & 10 \\
\hline
\end{tabular}


${ }^{1} \mathrm{H}$ NMR-7a

43047 yu340.10. fid
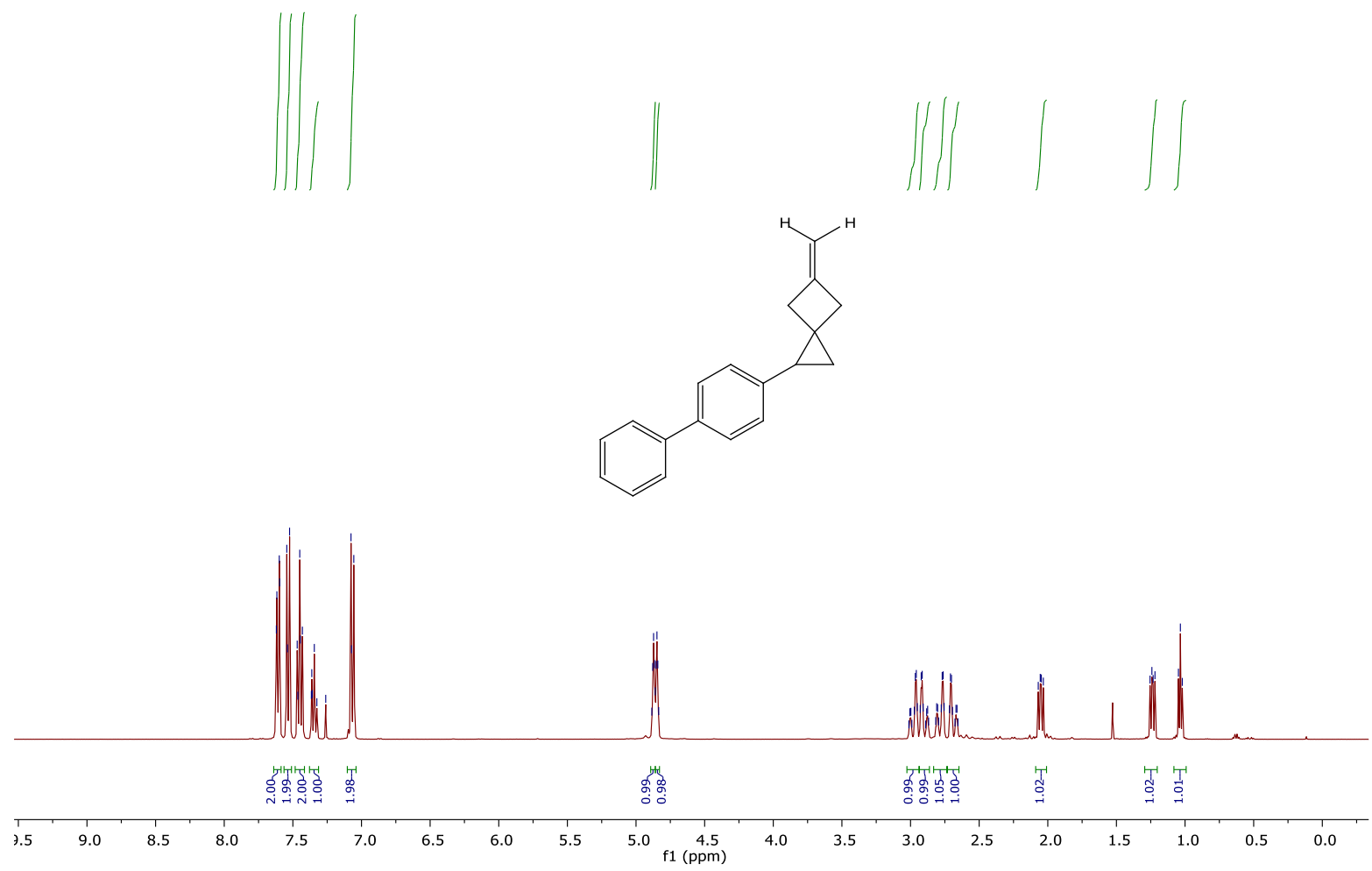

${ }^{13} \mathrm{C}$ NMR-7a

39925 yu280.11.fid

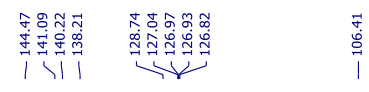

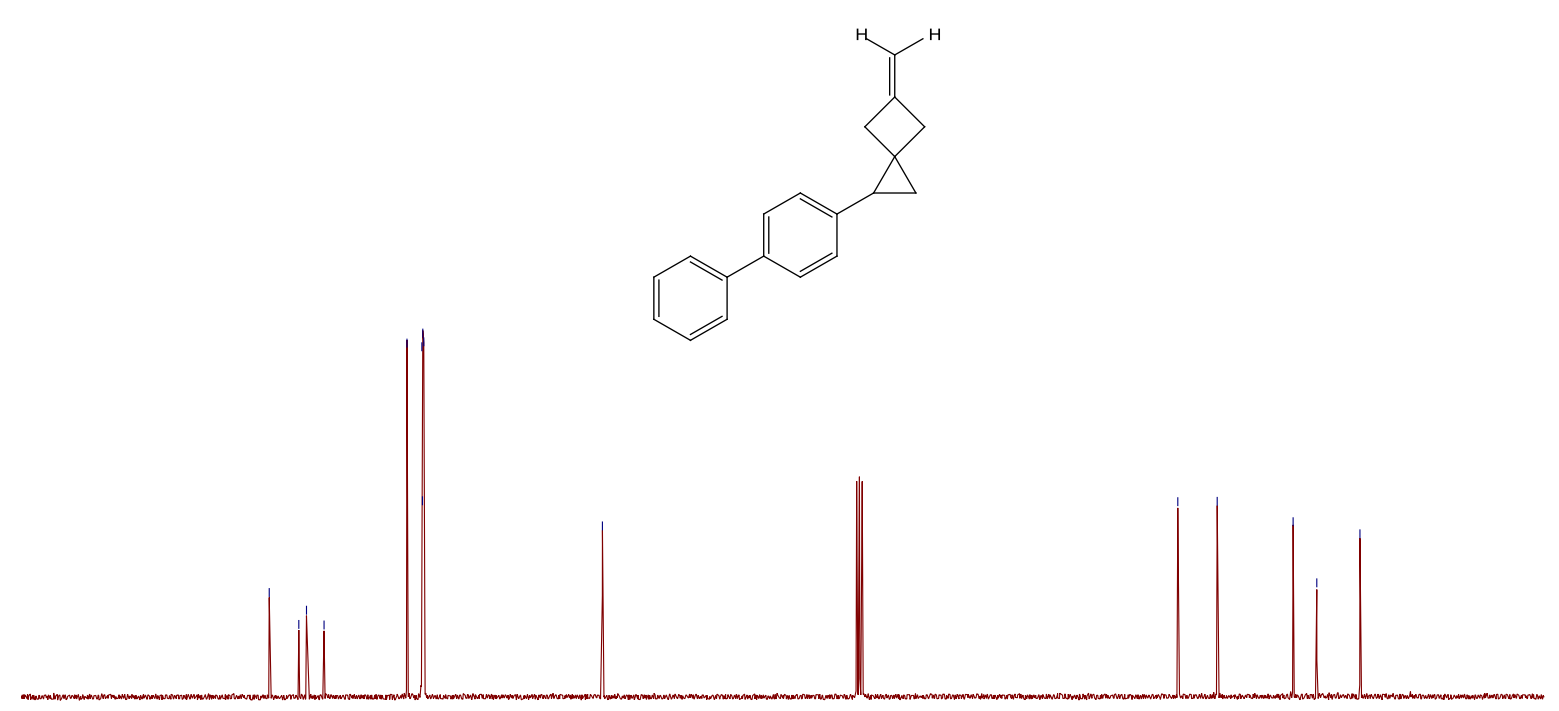

170

150

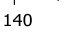

$130 \quad 120$

110

100

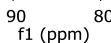

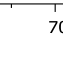

60

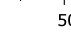




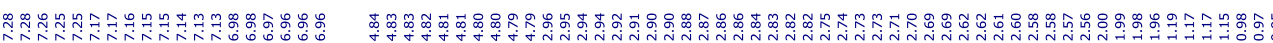
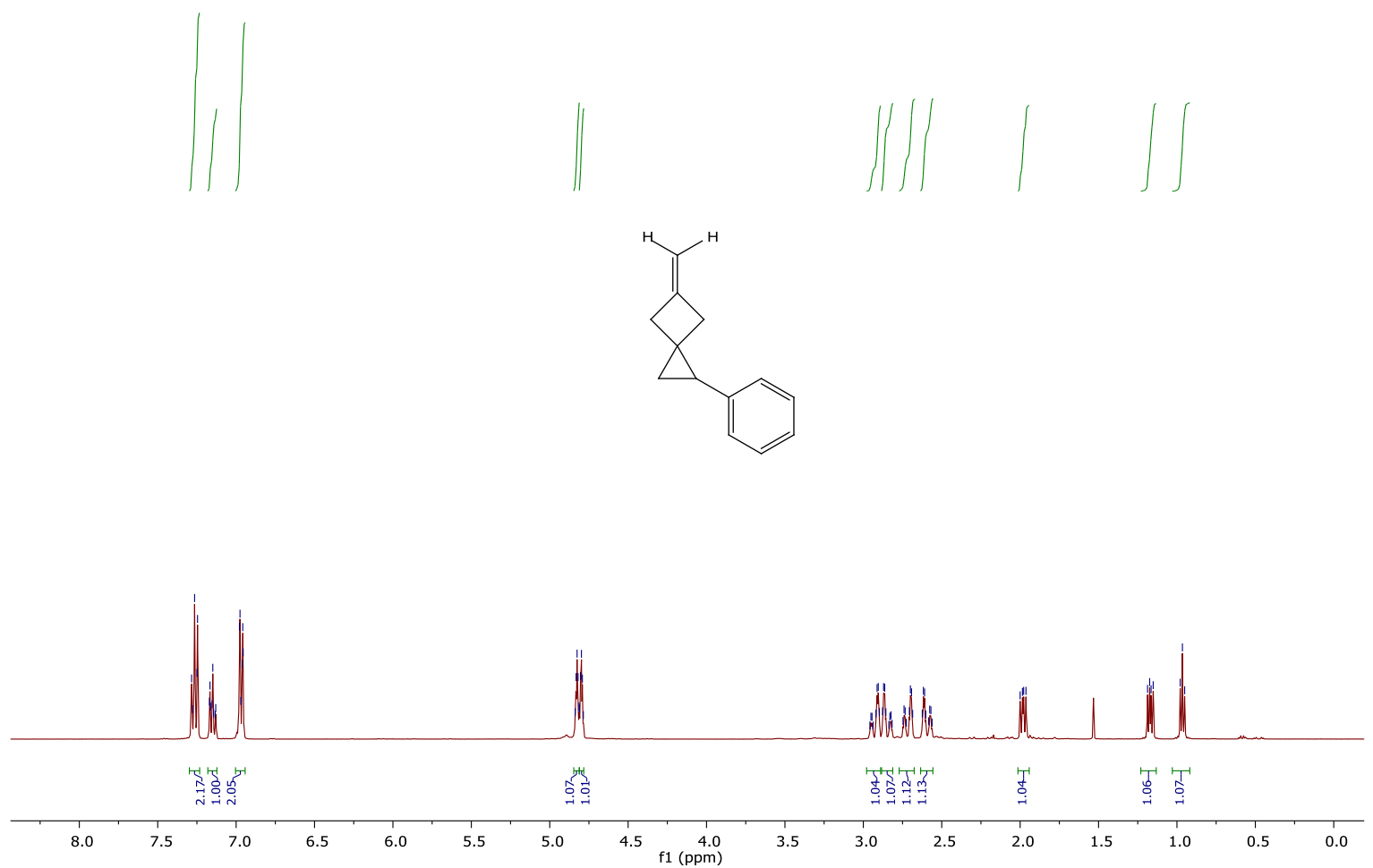

va/dn18877 yu343

${ }^{13} \mathrm{C}$ NMR-7b

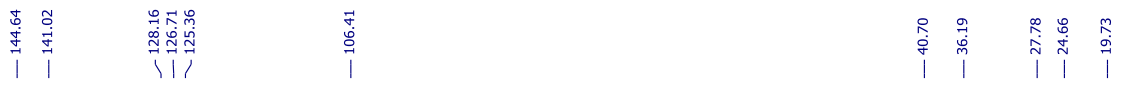
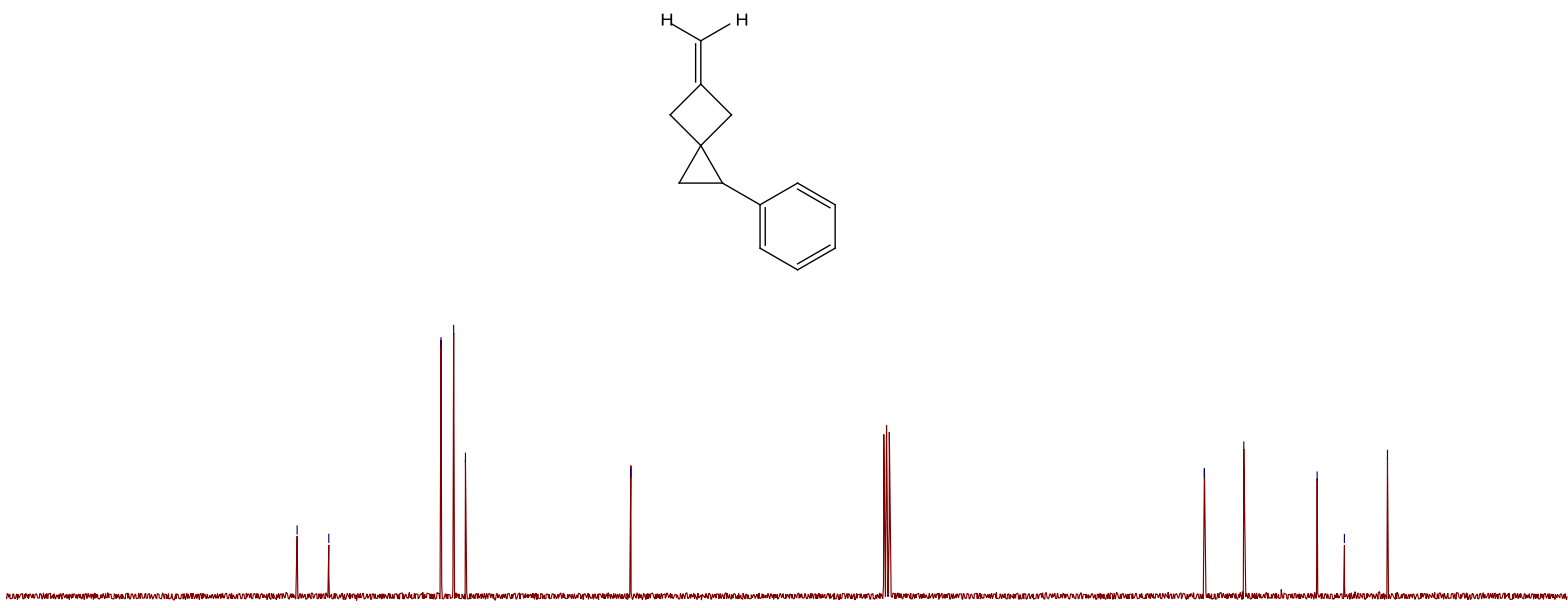

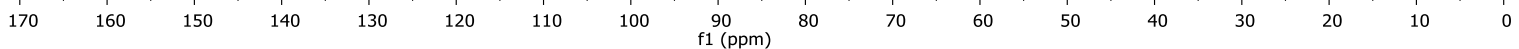




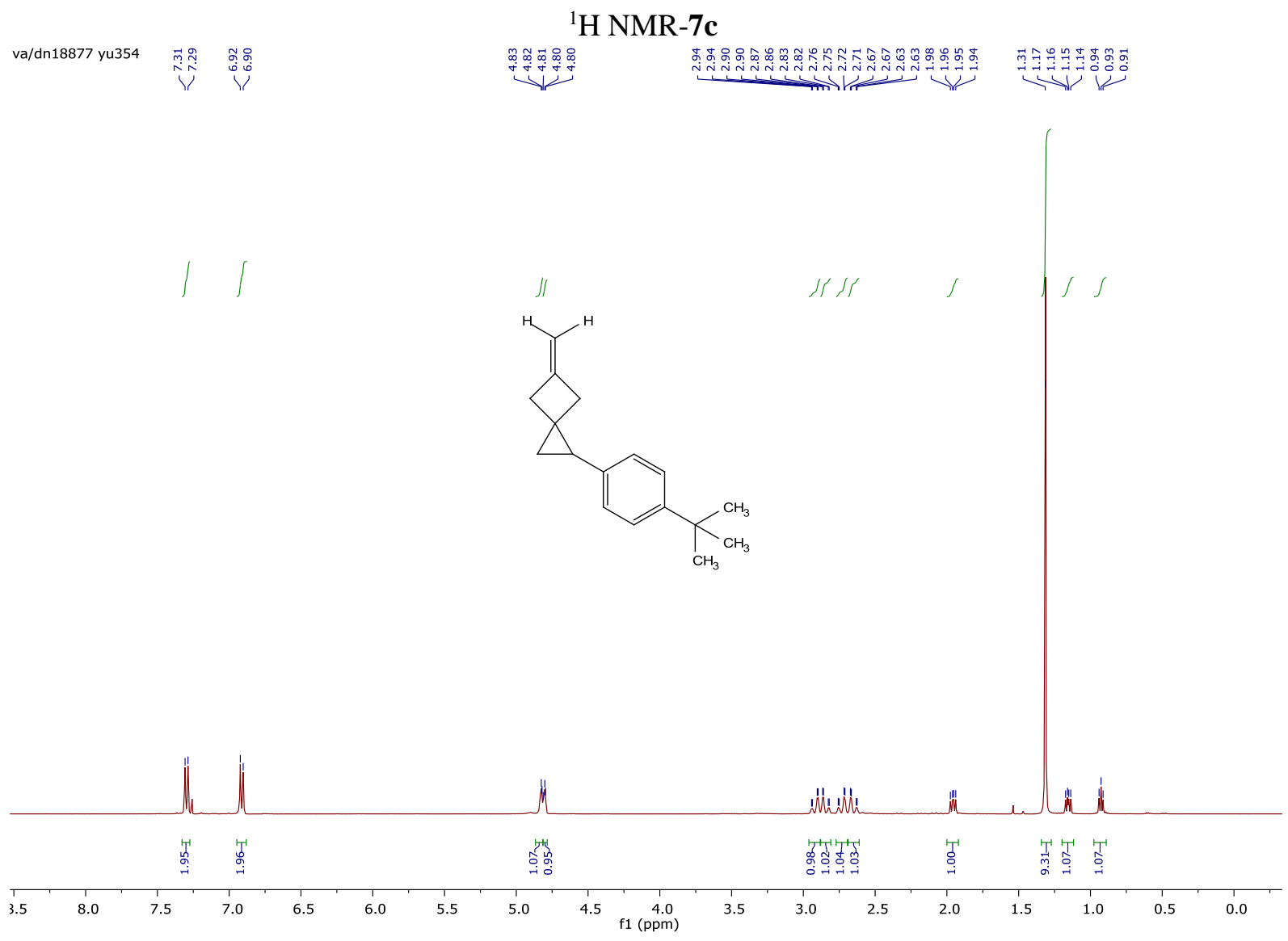

va/dn18877 yu354
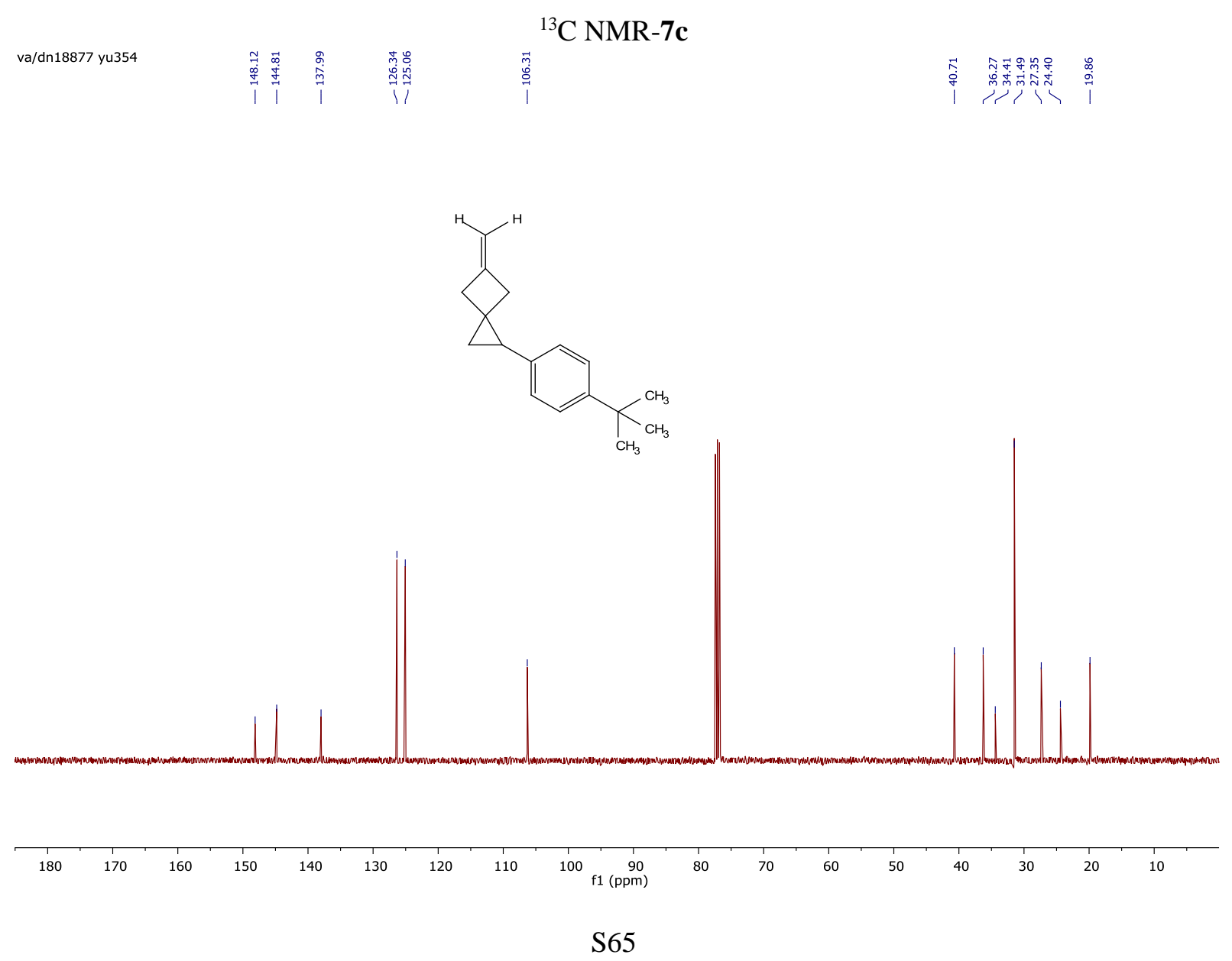


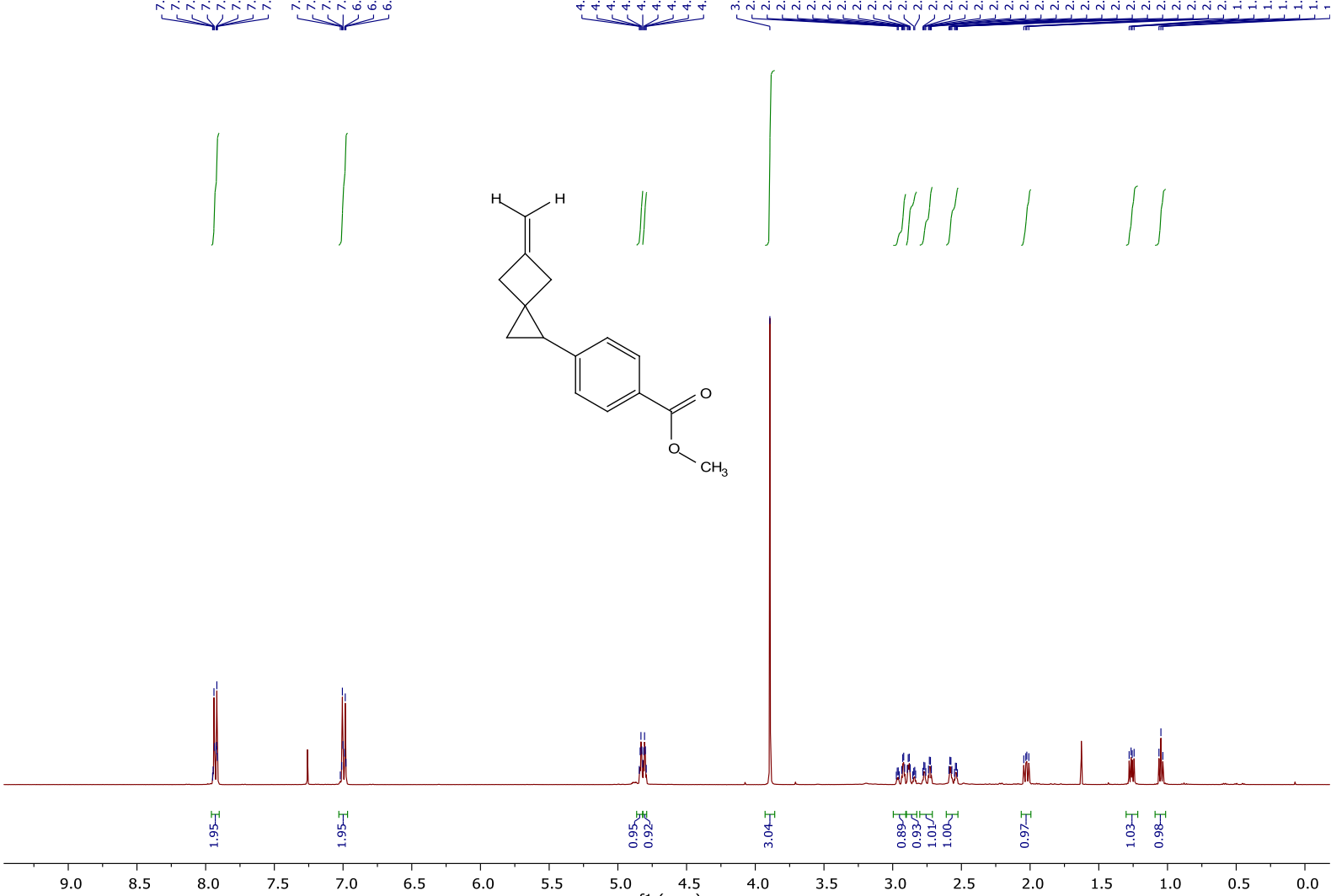

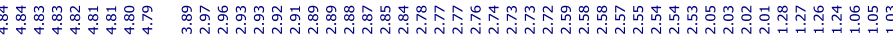

va/dn18877 yu365

${ }^{13}$ C NMR-7d

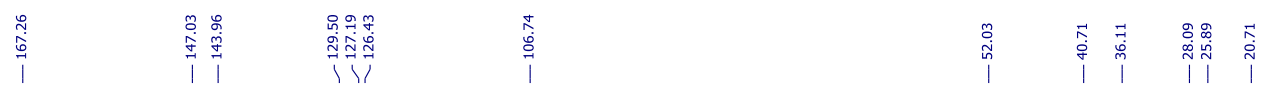
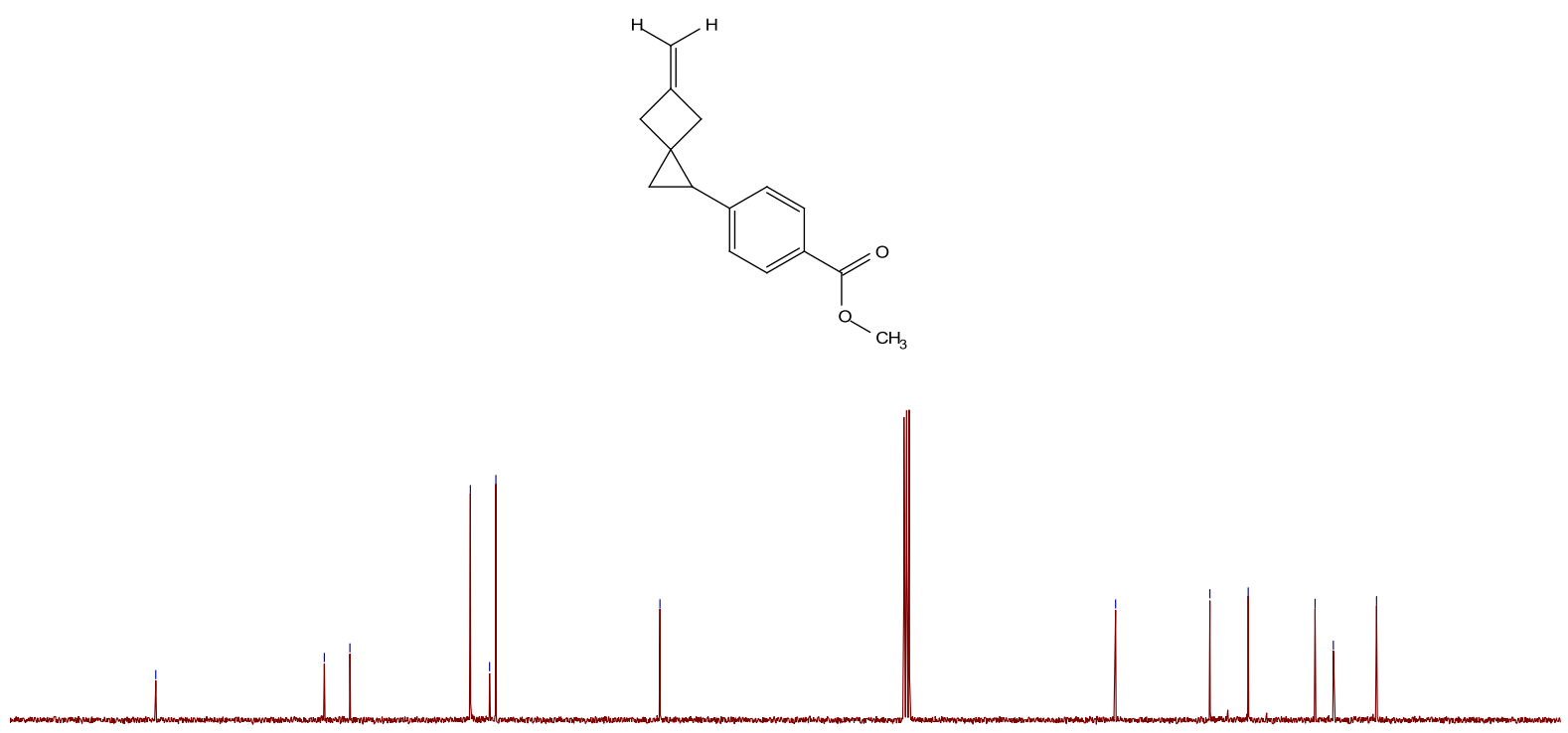

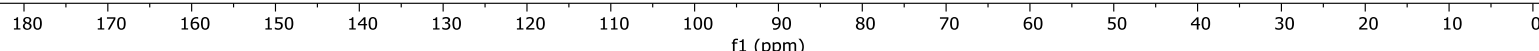


${ }^{1} \mathrm{H}$ NMR-7e
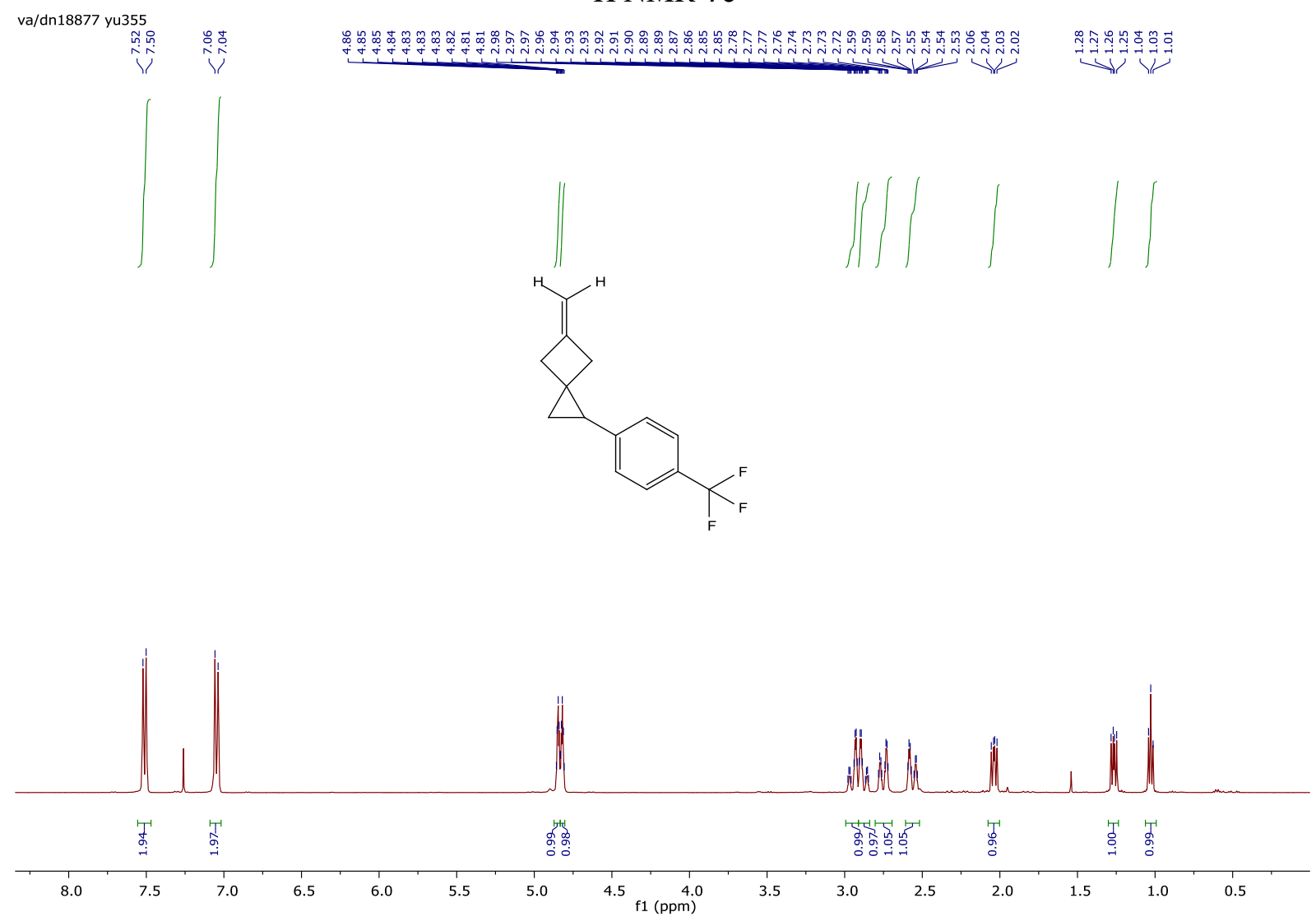

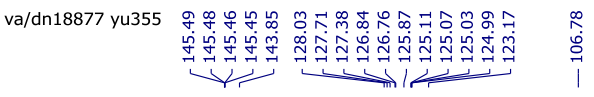

${ }^{13} \mathrm{C}$ NMR-7e
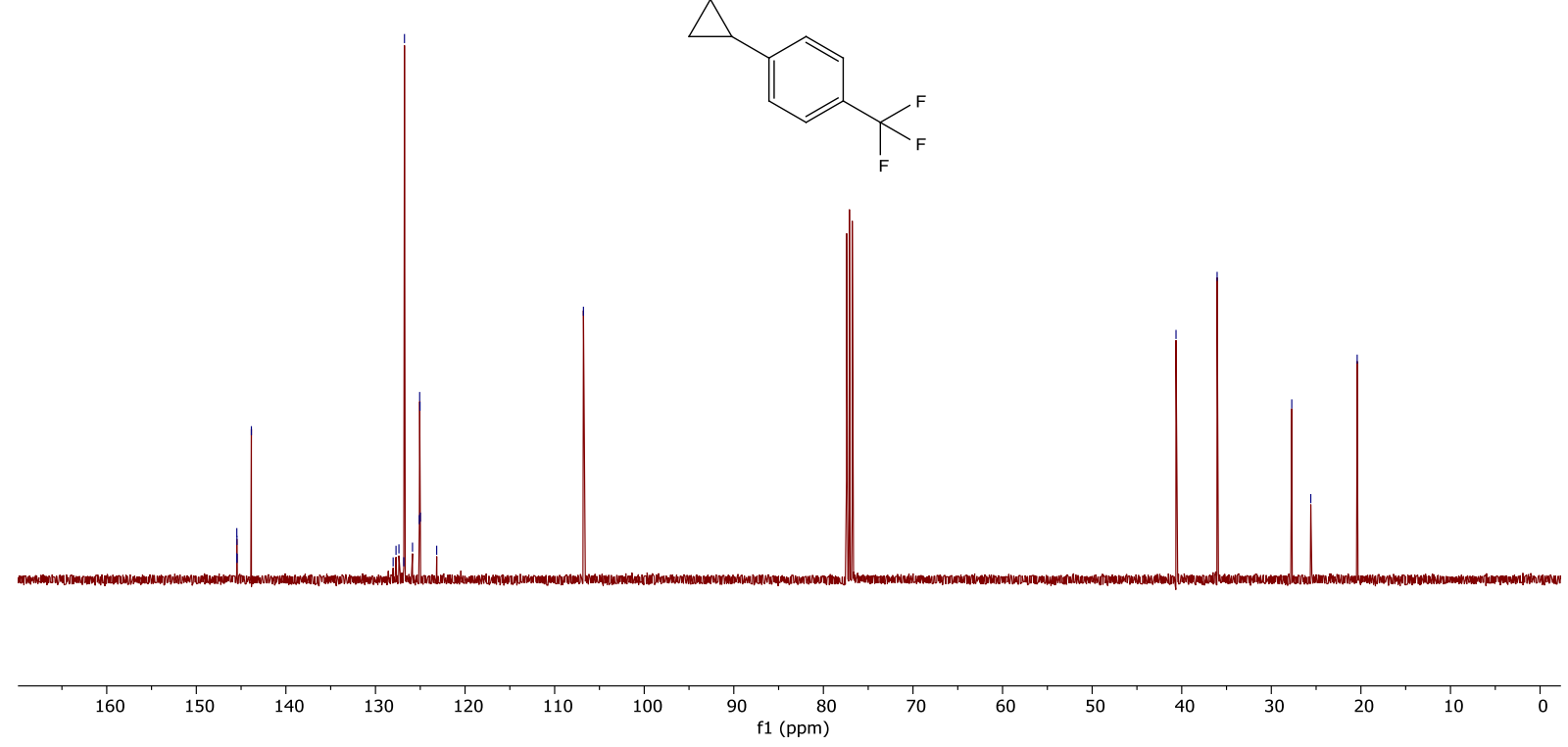

S67 
${ }^{19}$ F NMR-7e

va/dn18877 yu355
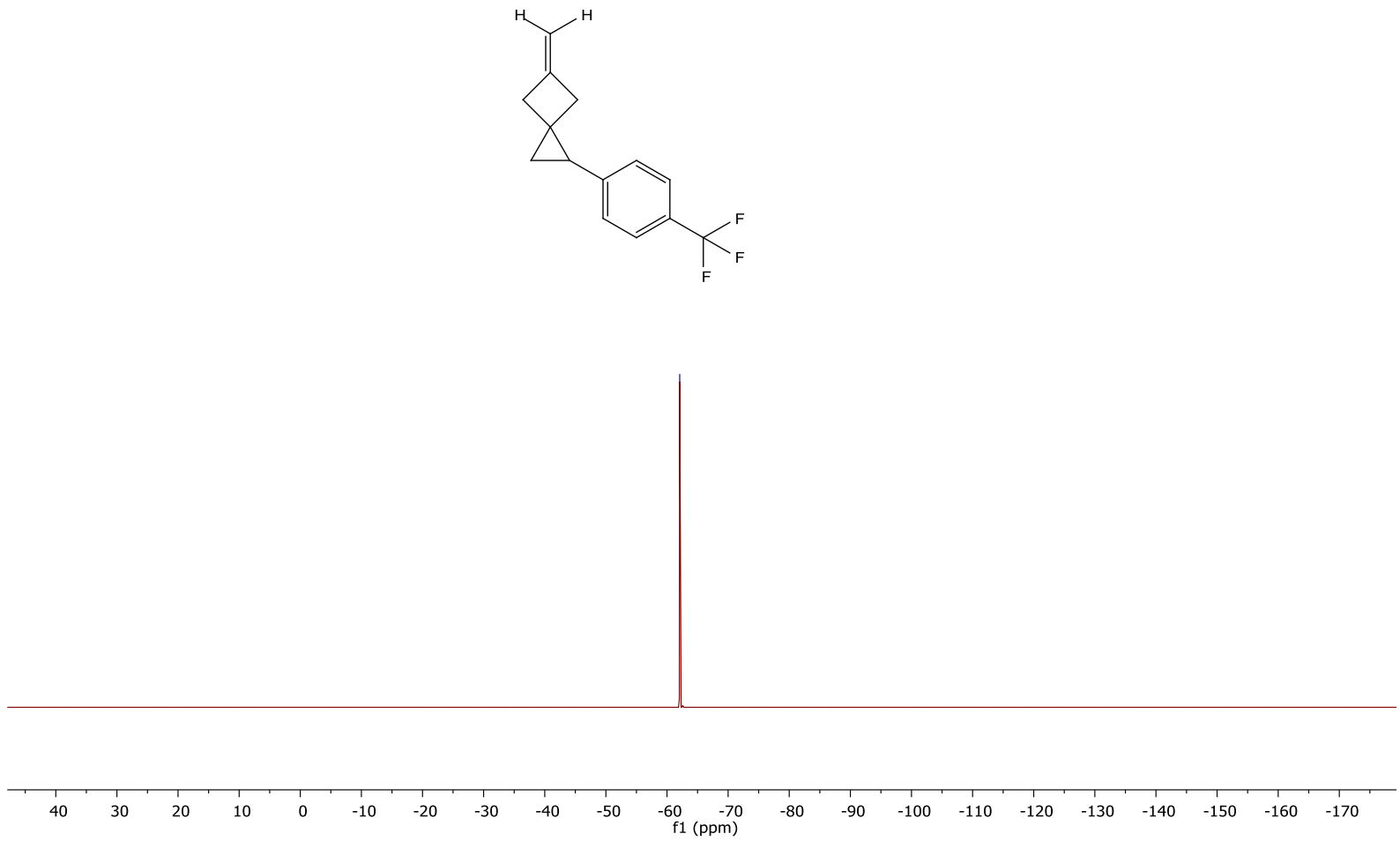

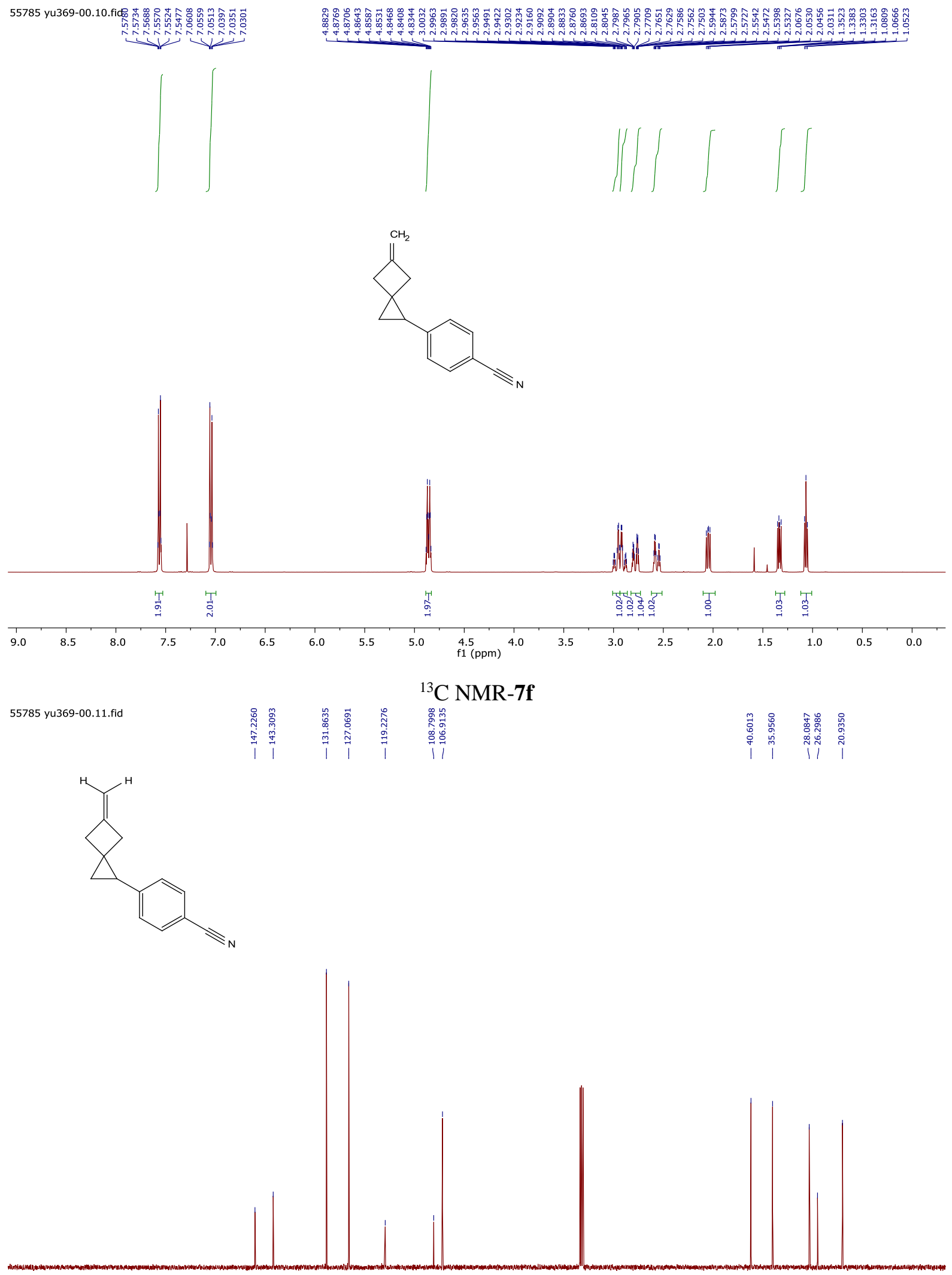

00

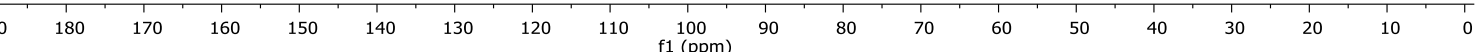


${ }^{1} \mathrm{H}$ NMR-7g

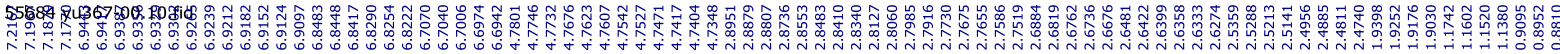
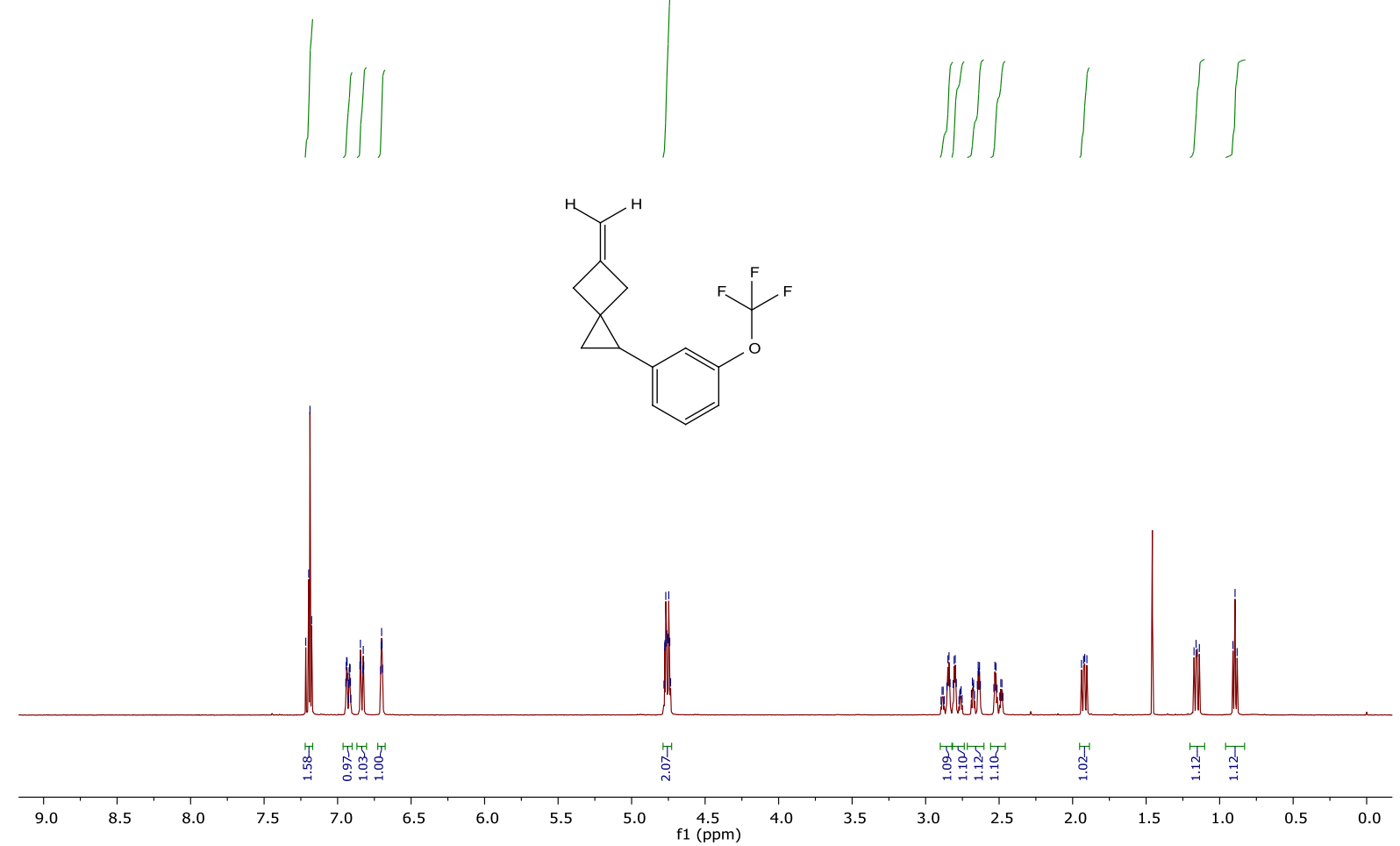

${ }^{13} \mathrm{C}$ NMR-7g

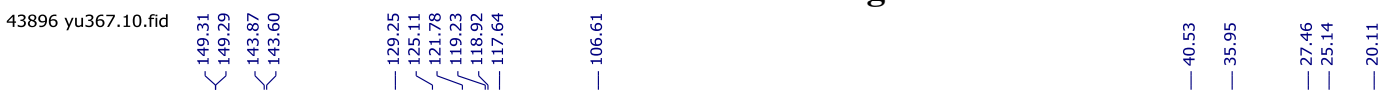
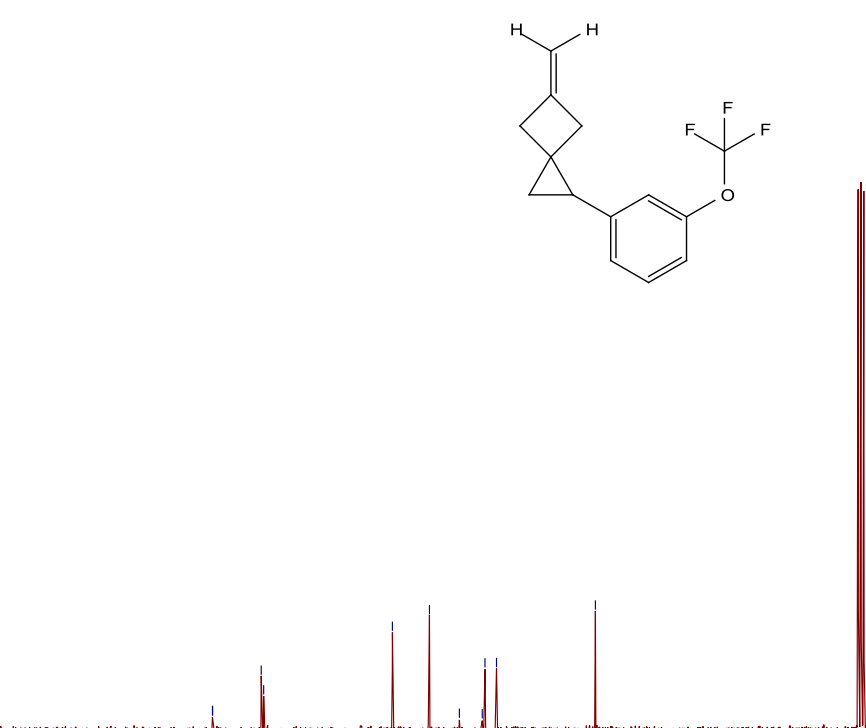

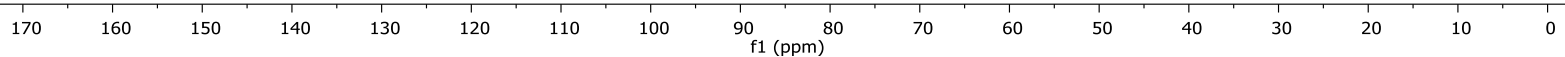


55677 y $376-00.11$ id

।

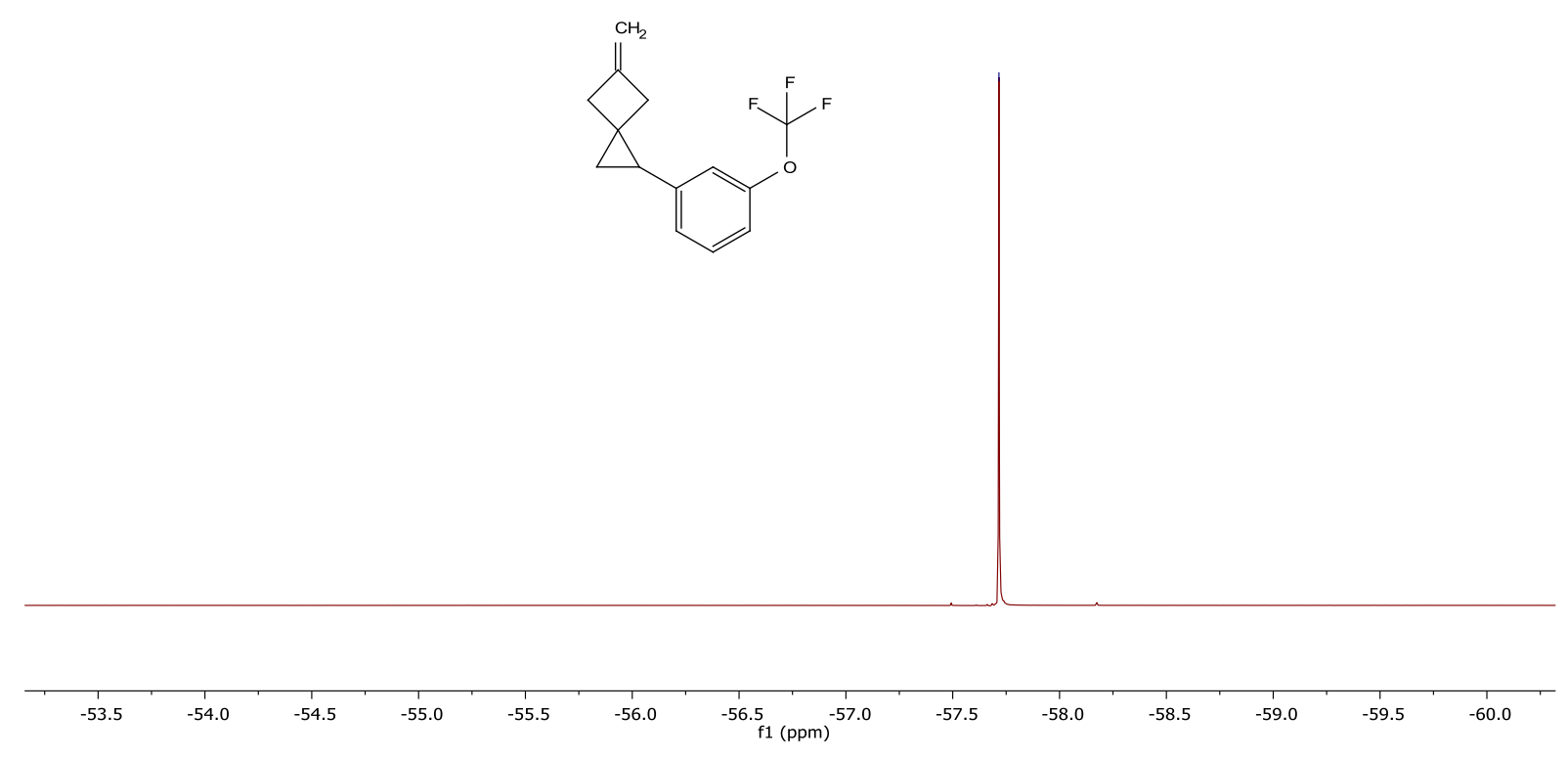


${ }^{1} \mathrm{H}$ NMR-7h

43364 yu350.10.fid

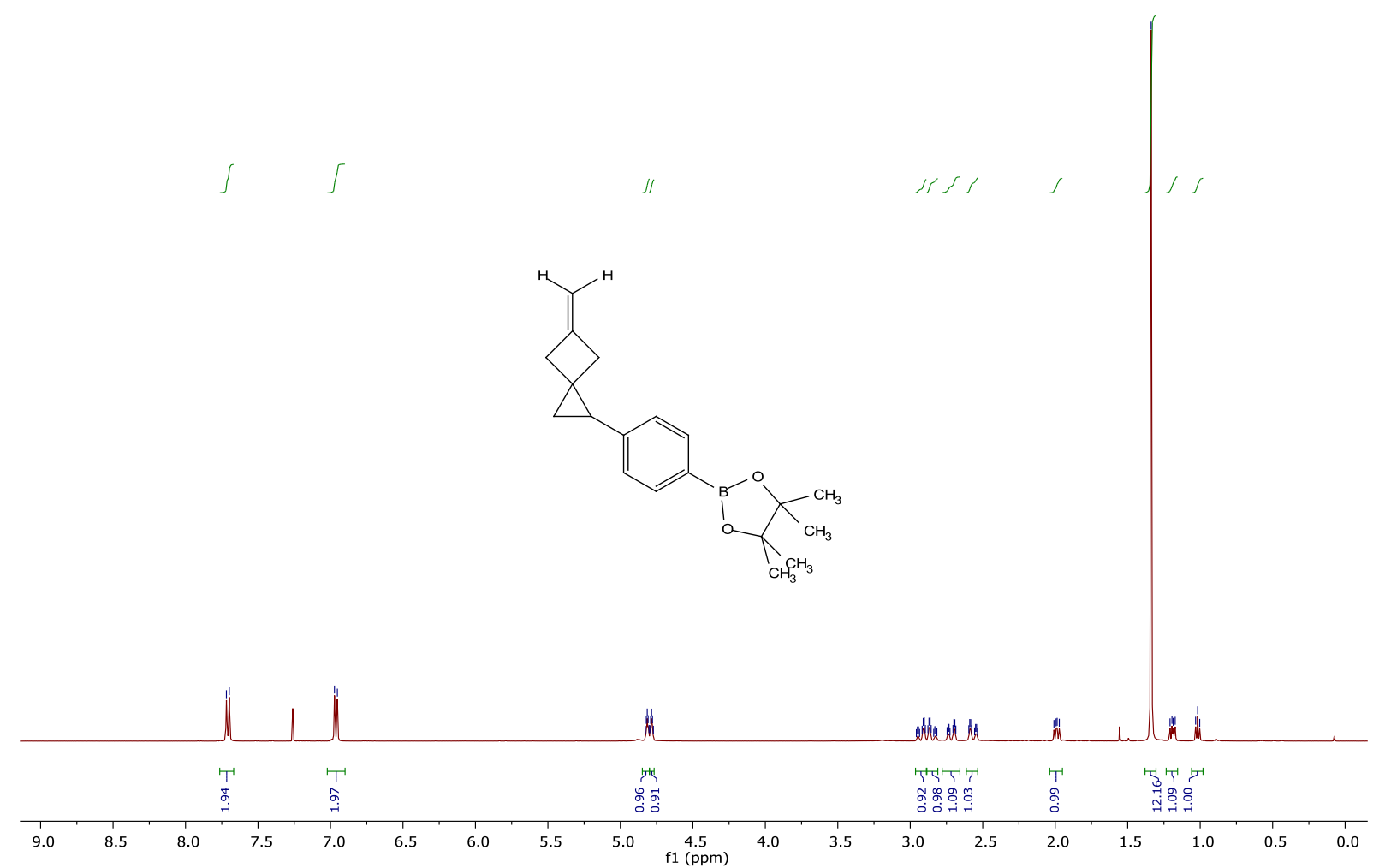

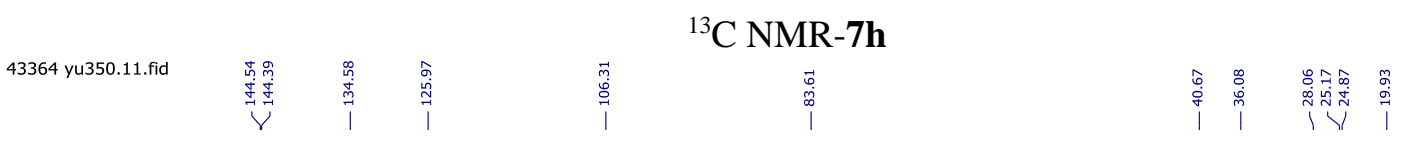
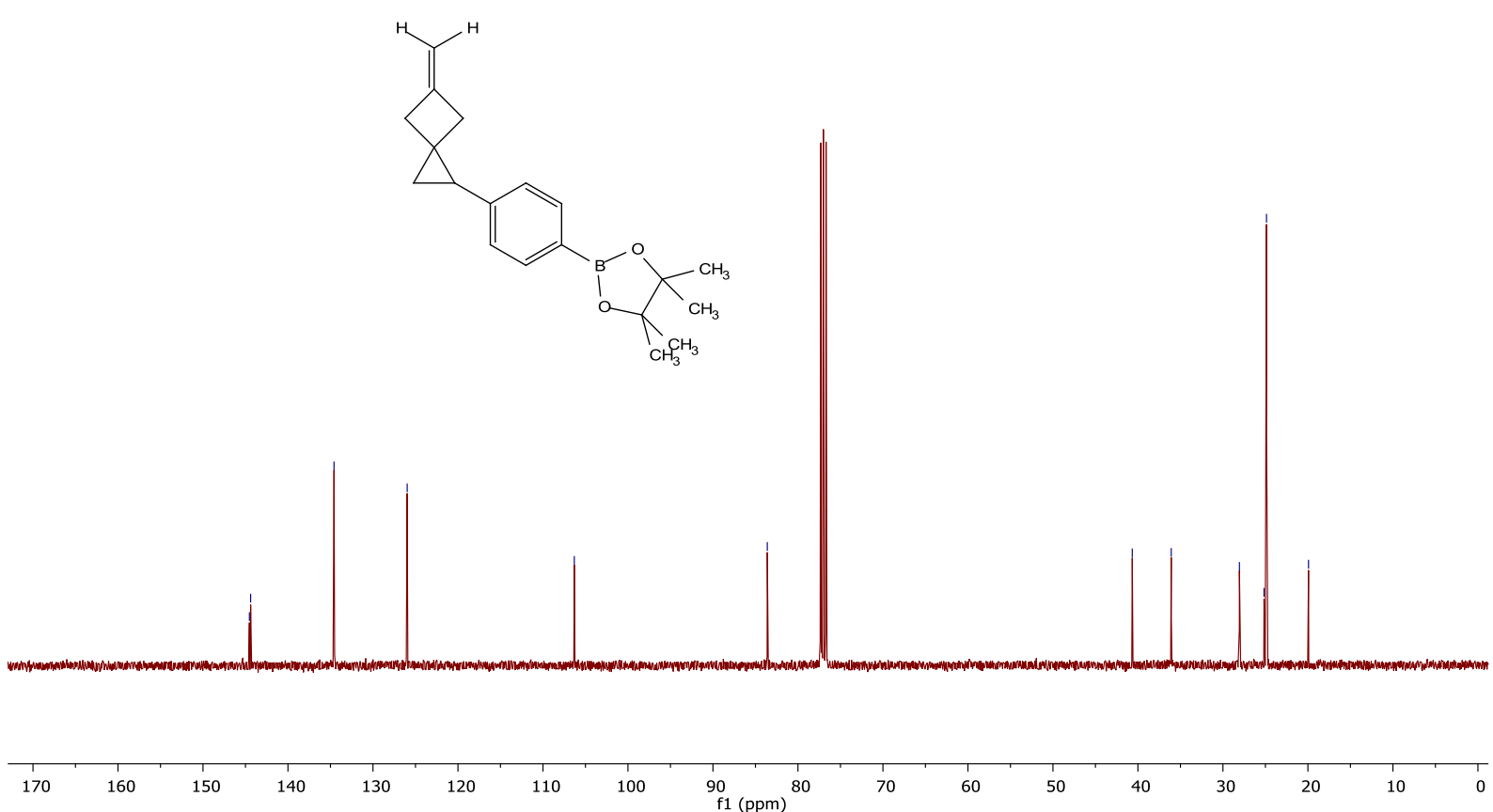


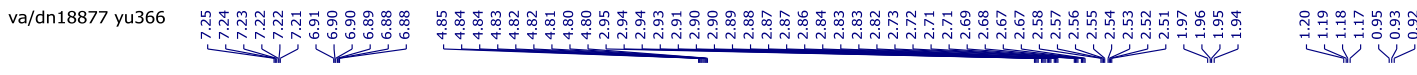
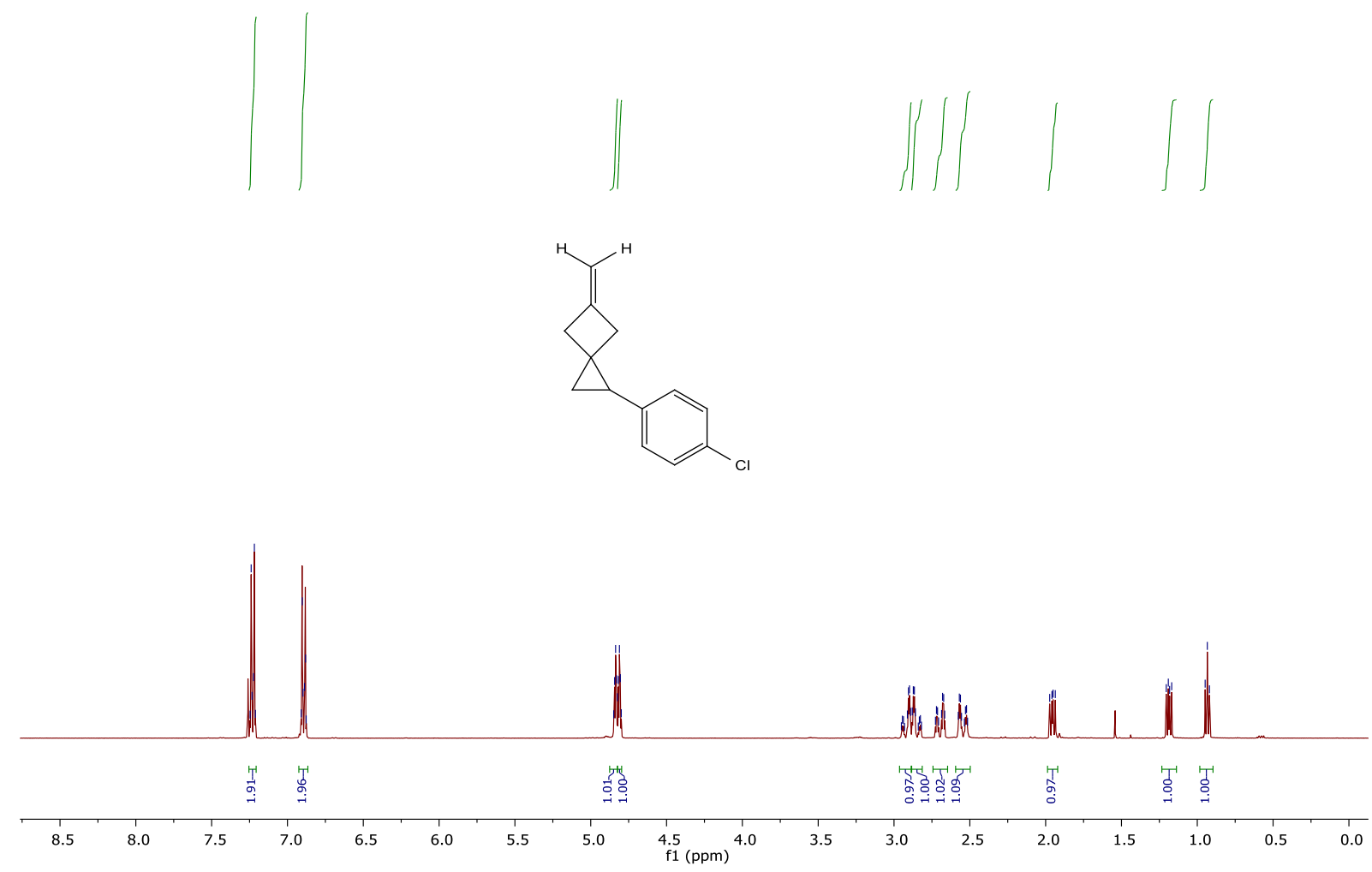

${ }^{13}$ C NMR-7i

va/dn18877 yu366

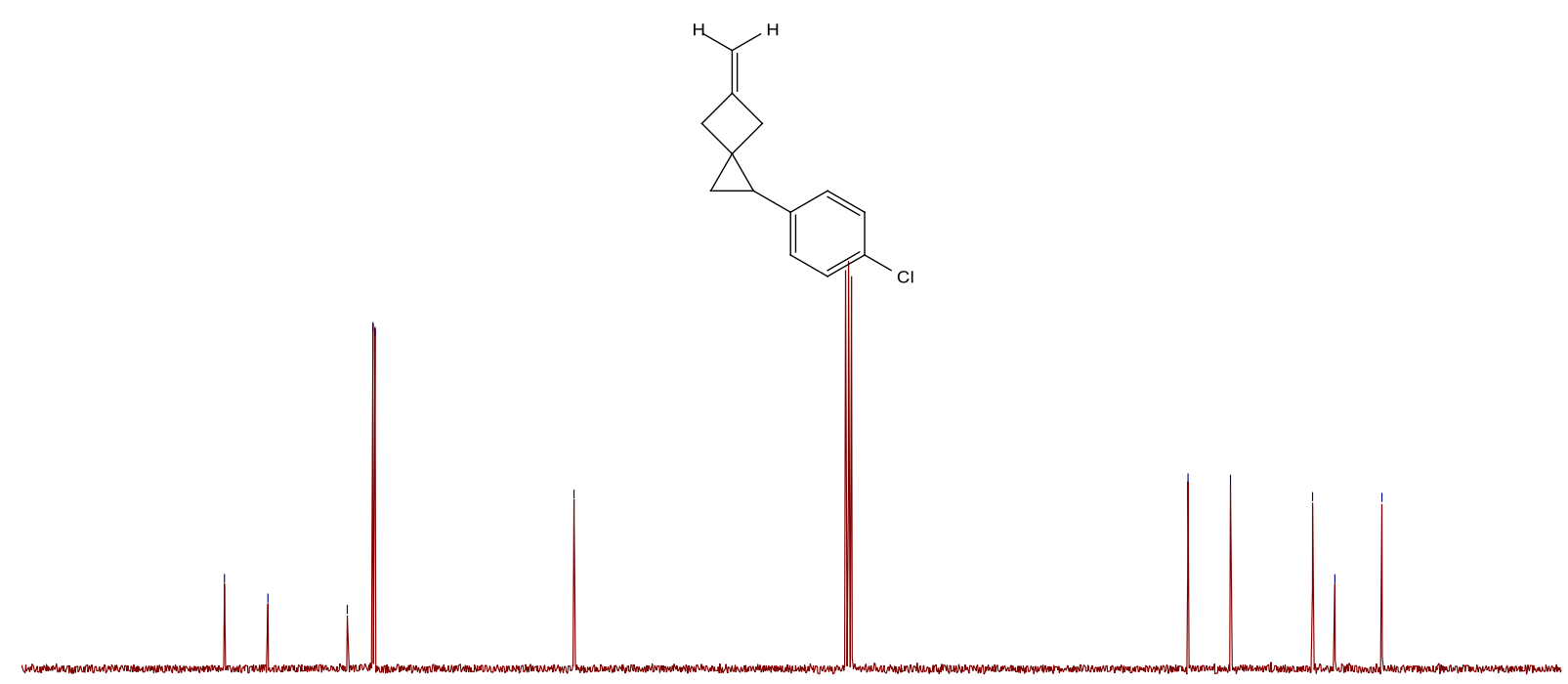

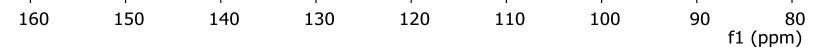


${ }^{1} \mathrm{H}$ NMR-7j

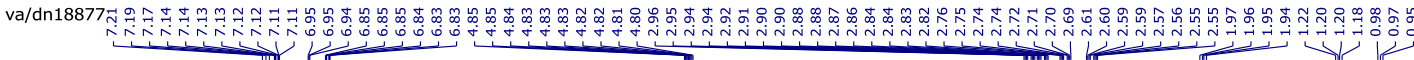
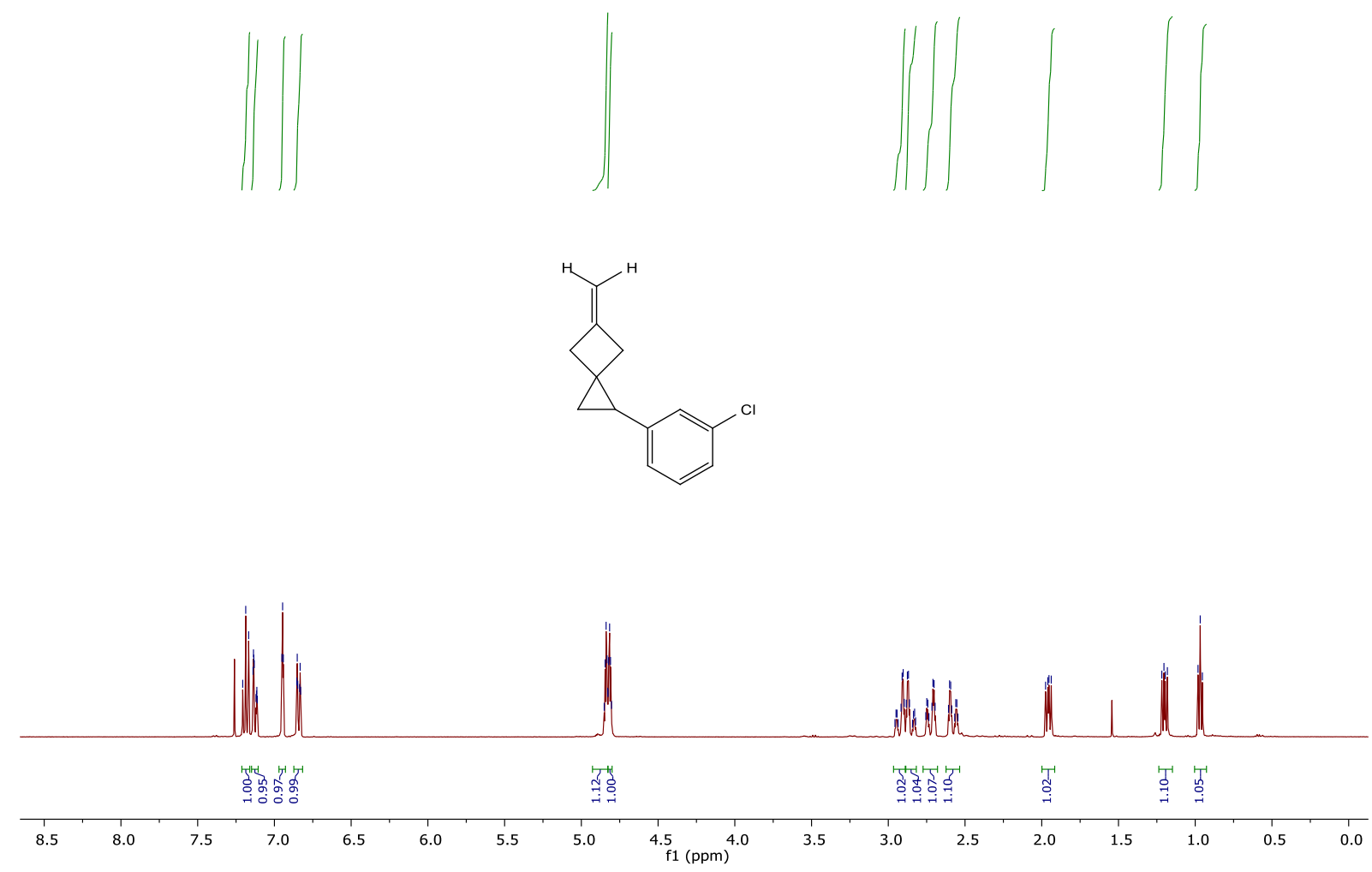

$\mathrm{va} / \mathrm{dn} 18877$

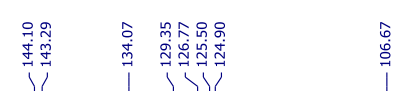

${ }^{13}$ C NMR-7j
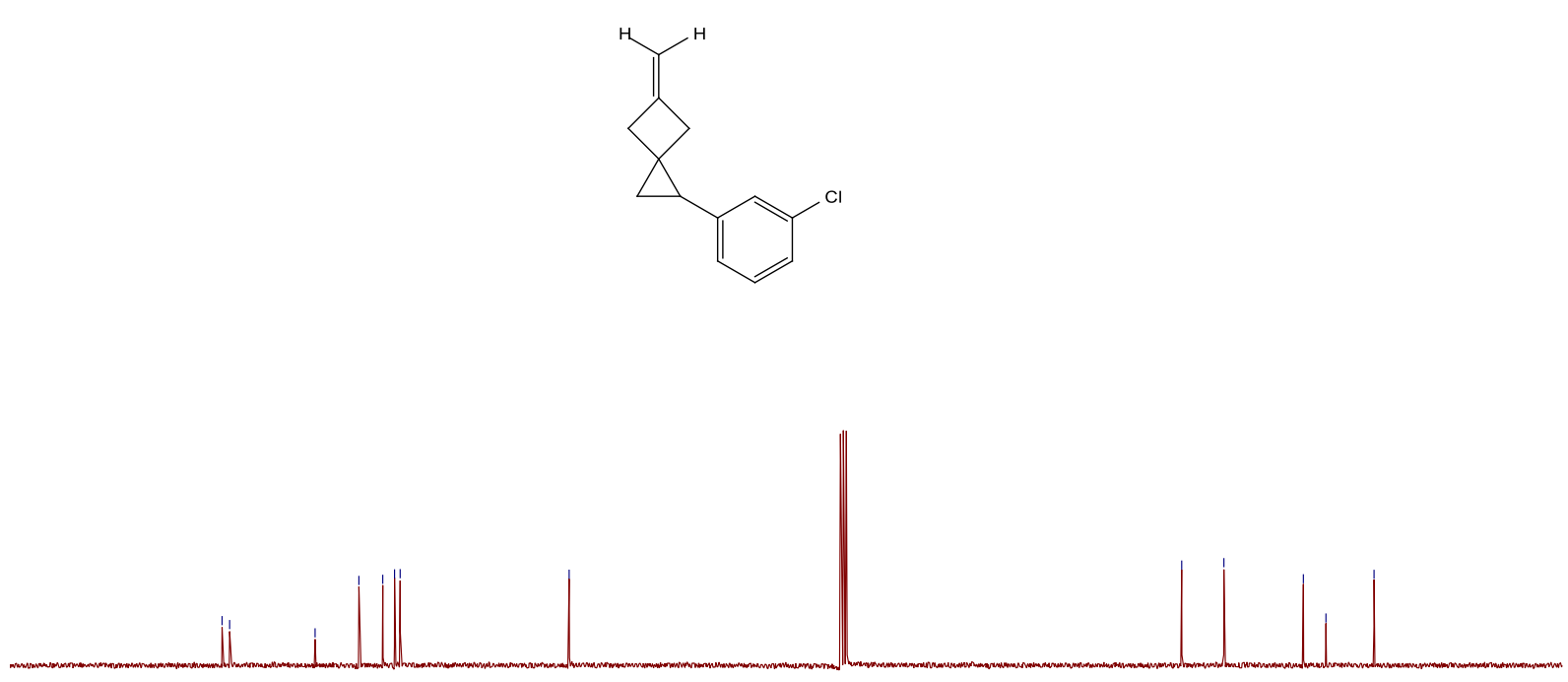

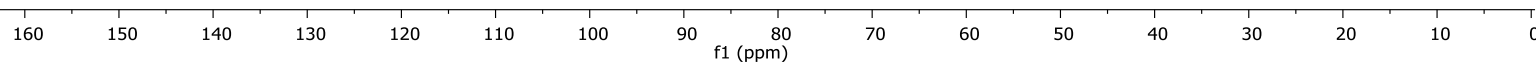


${ }^{1} \mathrm{H}$ NMR-7k

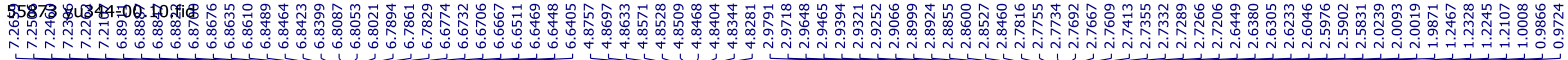
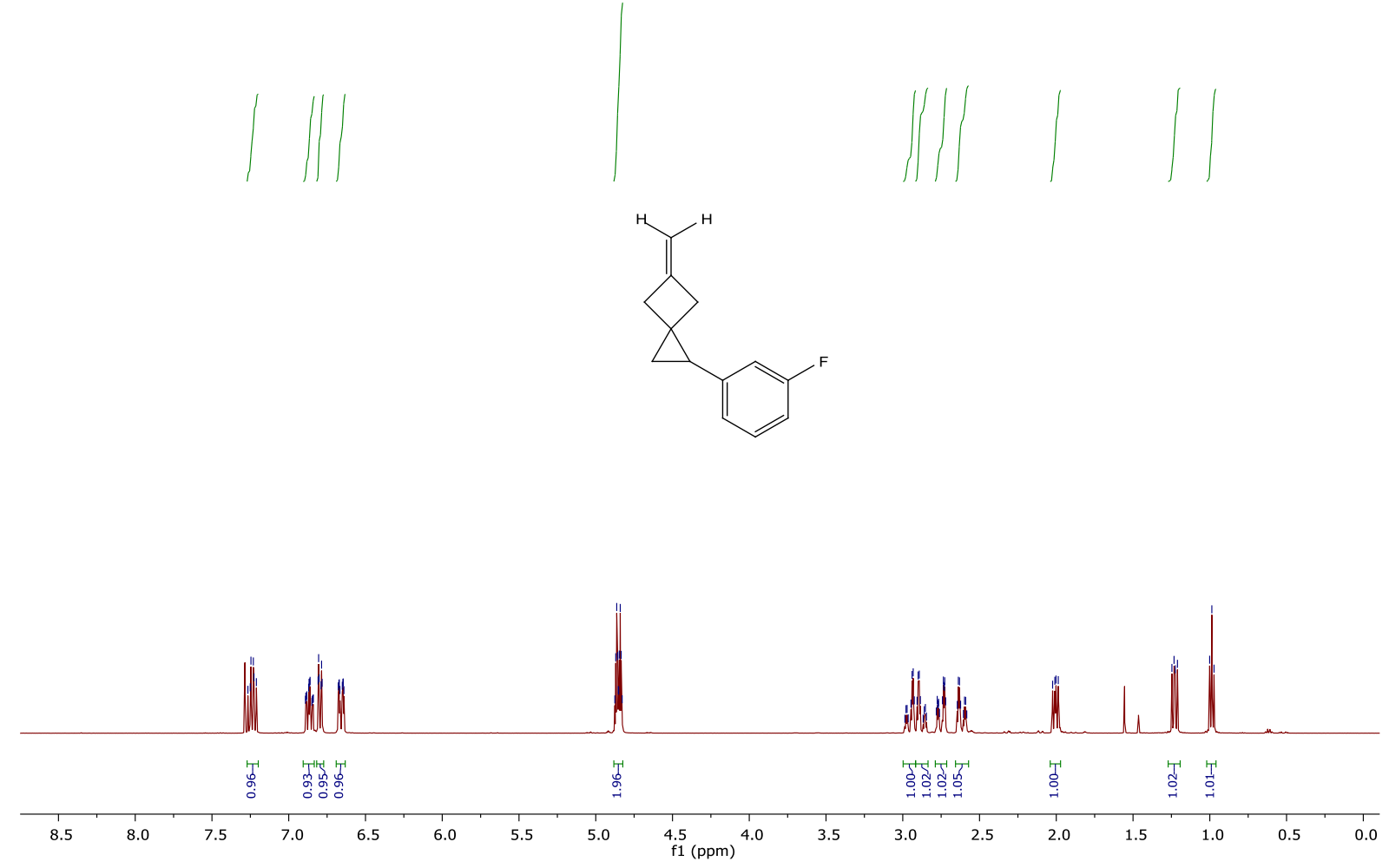

${ }^{13} \mathrm{C}$ NMR-7k

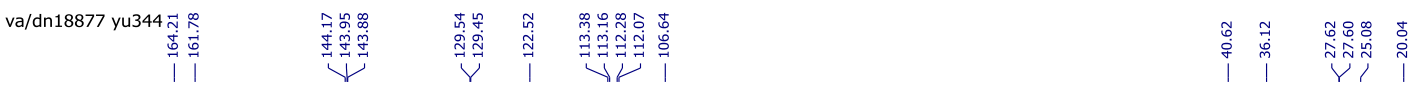
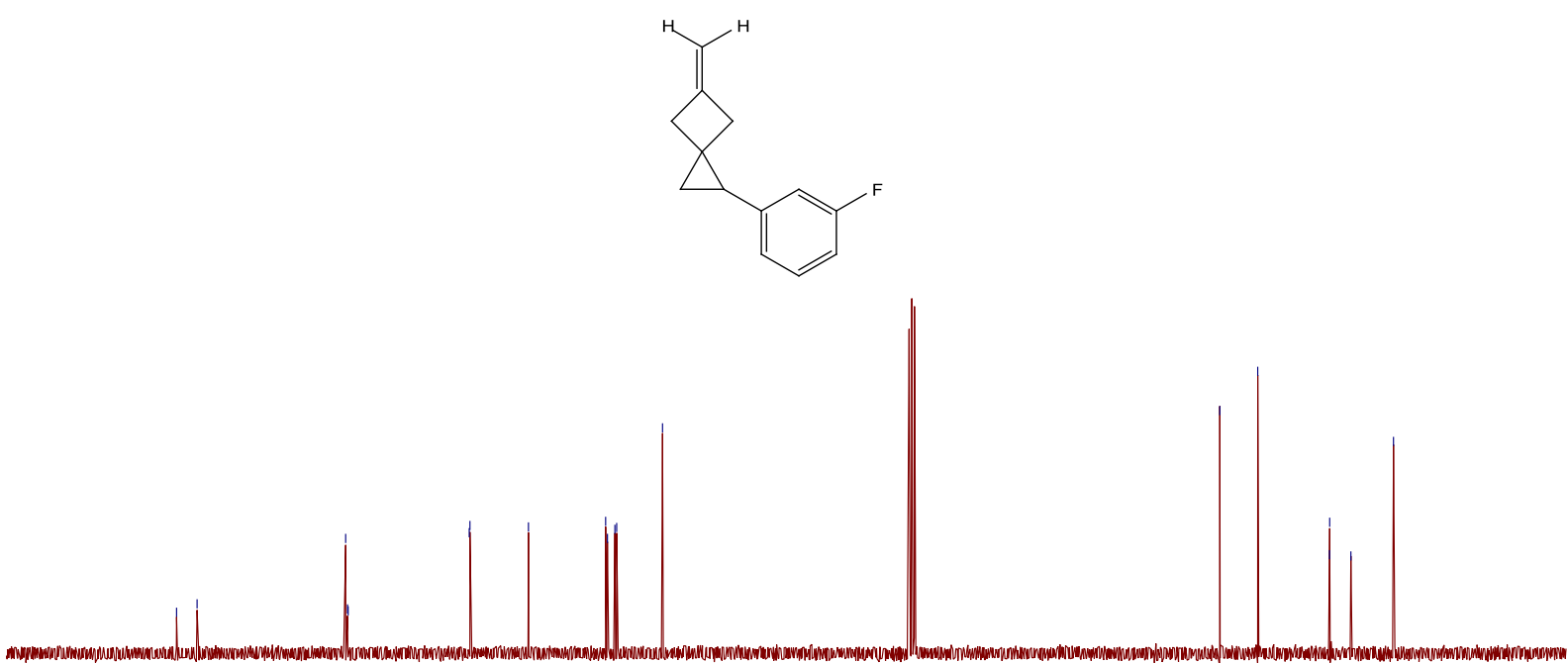

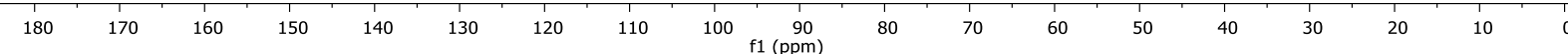


${ }^{19}$ F NMR-7k

va/dn18877 yu344

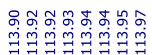

국구국구국

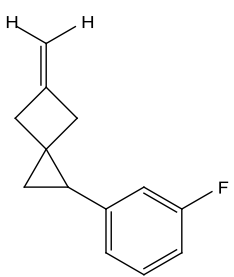

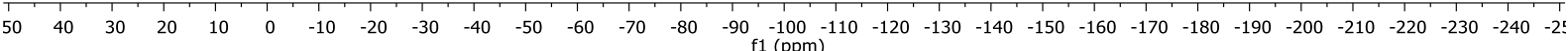



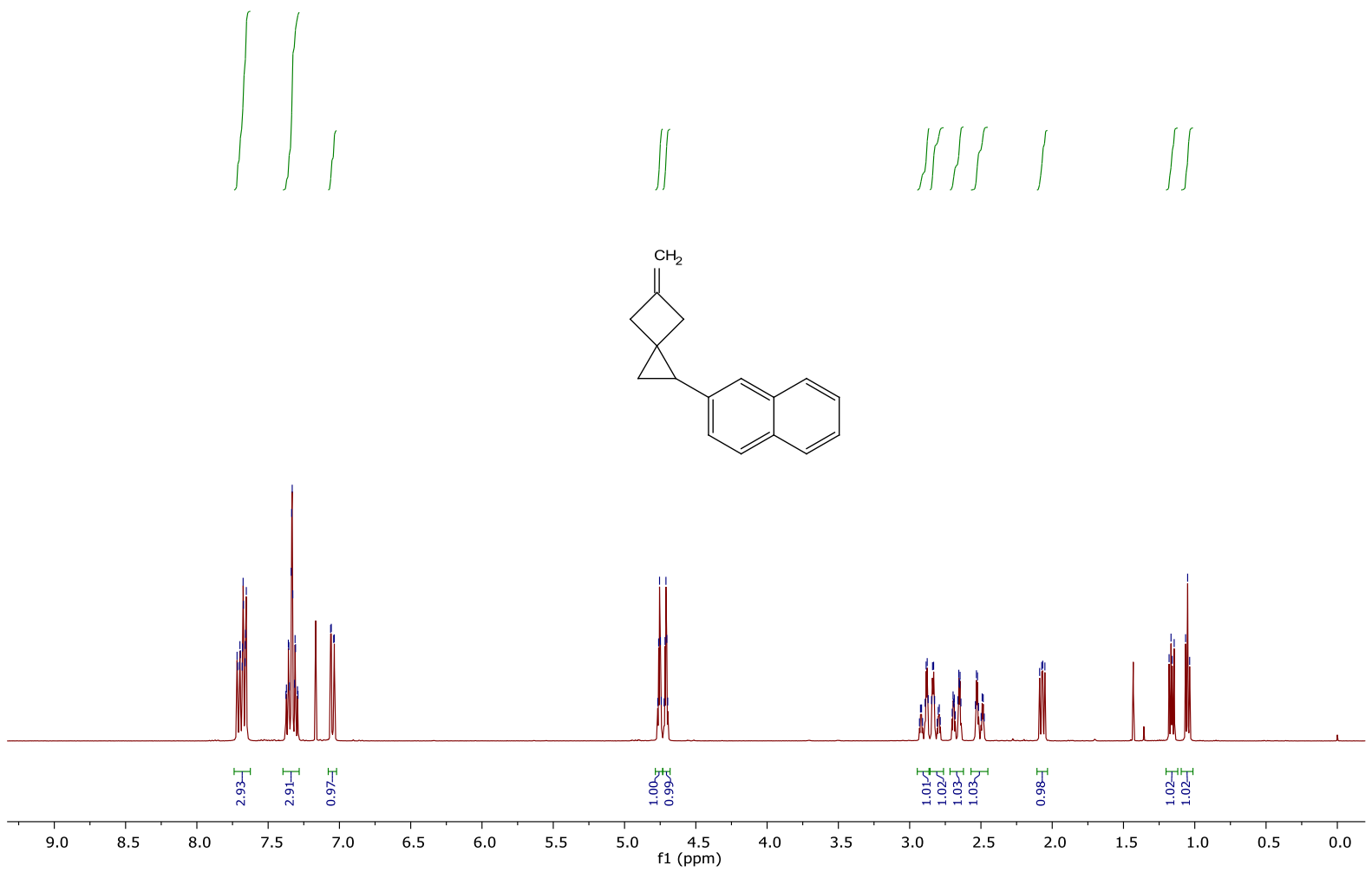

55683 yu357-00.11.fid

${ }^{13}$ C NMR-7l

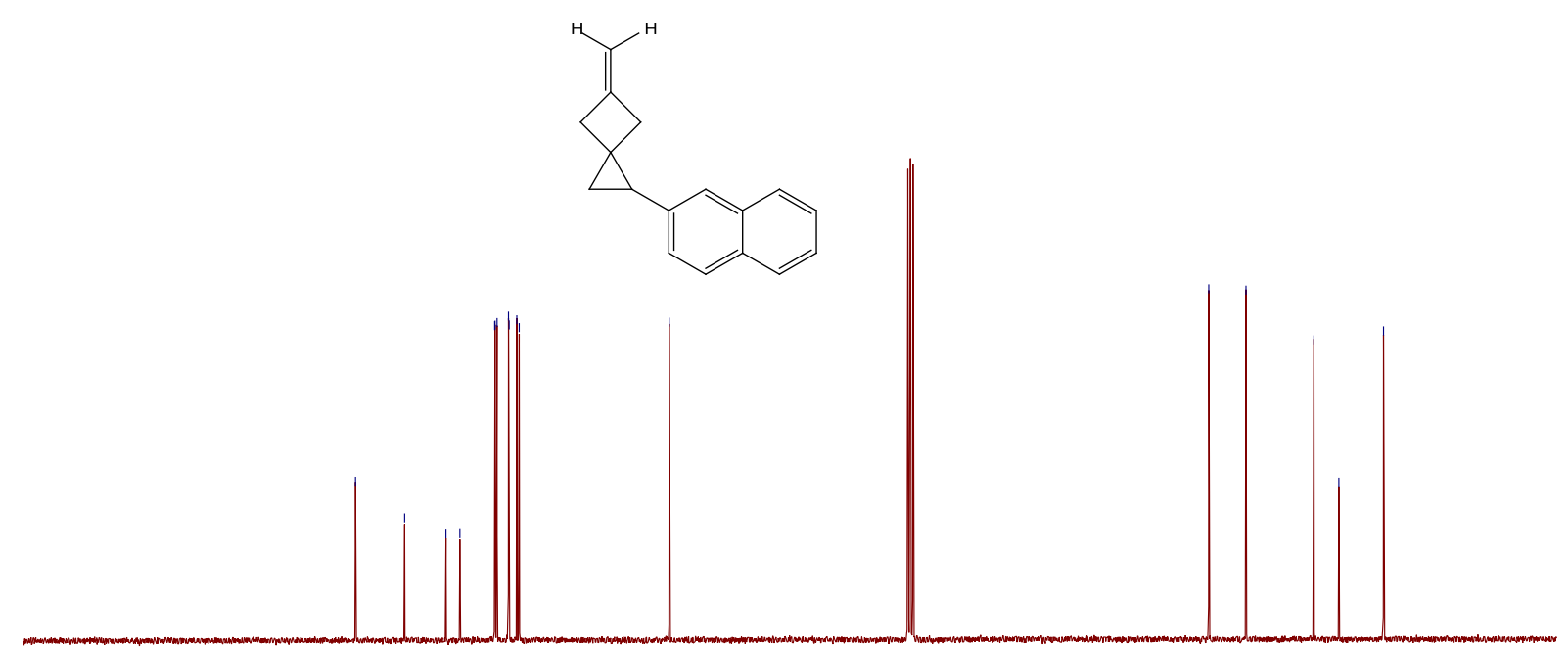

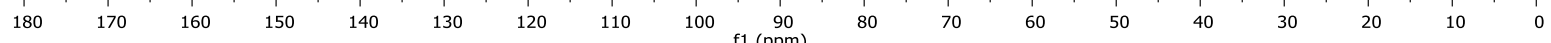


${ }^{1} \mathrm{H}$ NMR-7m

44816 yu383.10.fid

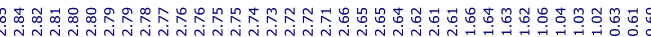

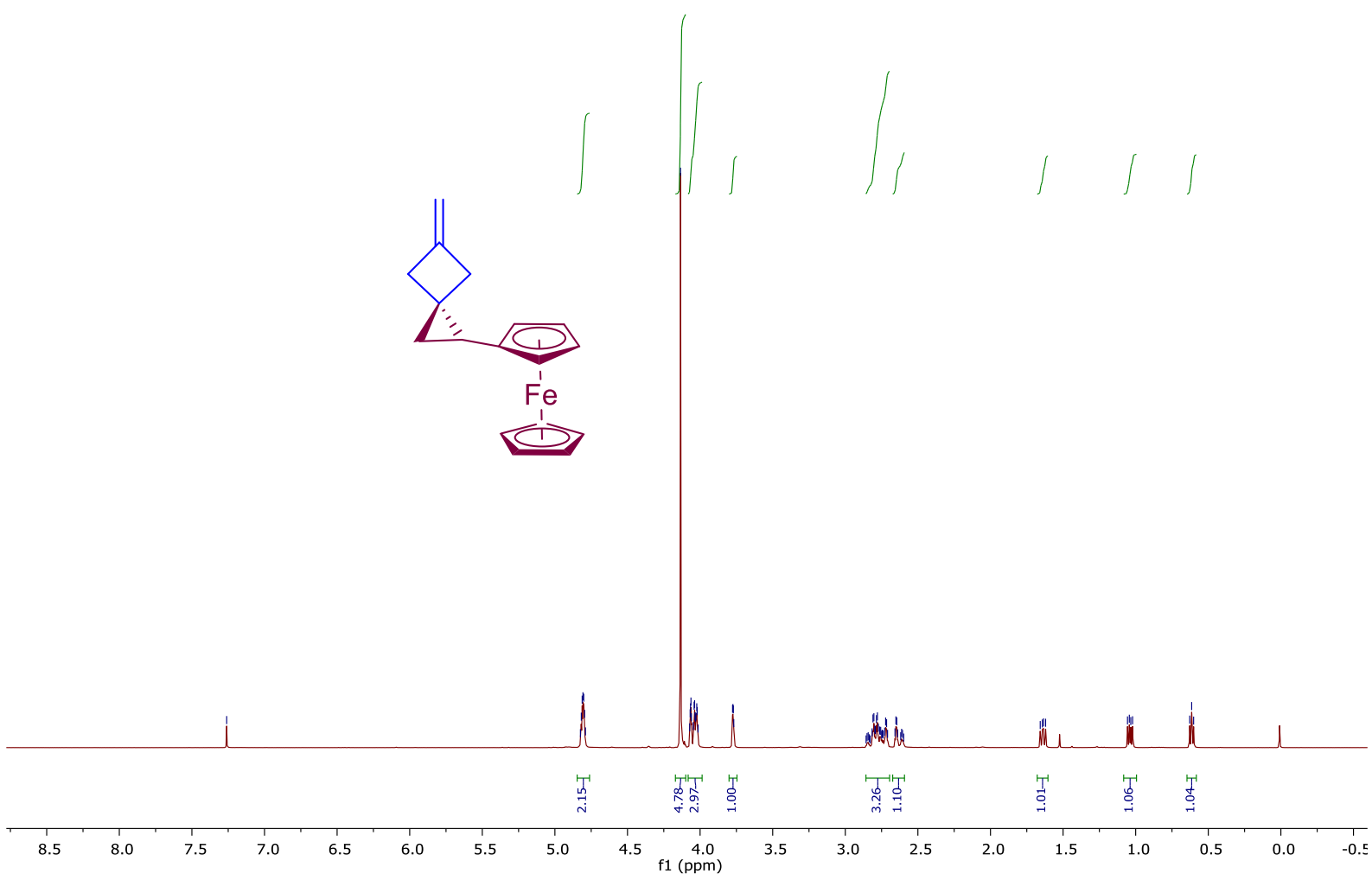

44816 yu383.11.fid

$\underset{\substack{\infty \\ \dot{j}}}{\substack{j \\ \mid}}$

${ }^{13} \mathrm{C}$ NMR-7m
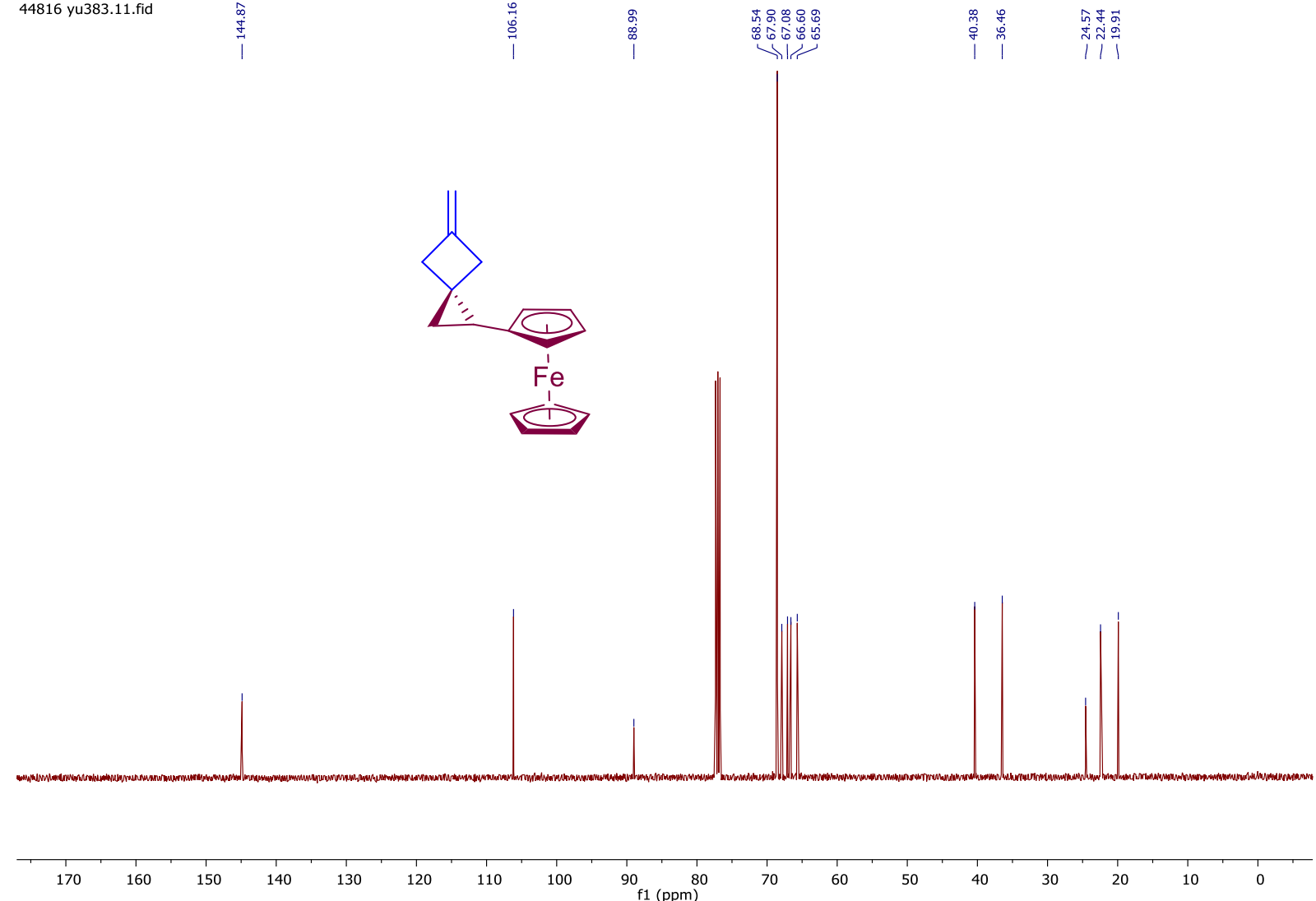
${ }^{1} \mathrm{H}$ NMR-7n

45185 yu395.10.fid

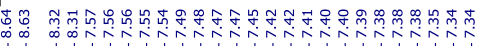

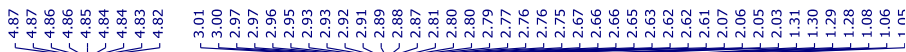
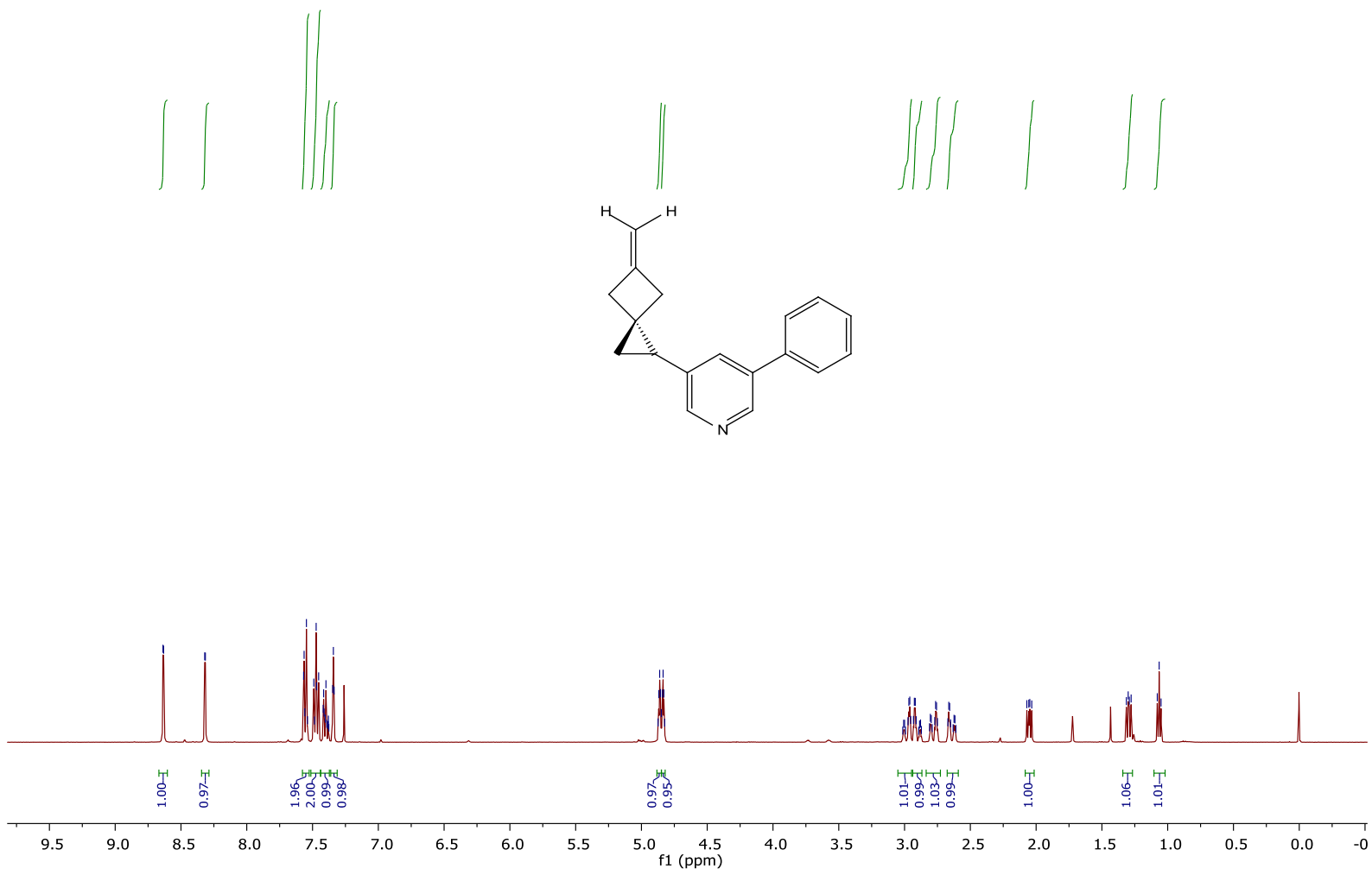

${ }^{13}$ C NMR-7n

$\mathrm{va} / \mathrm{dn} 18877$

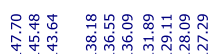

110
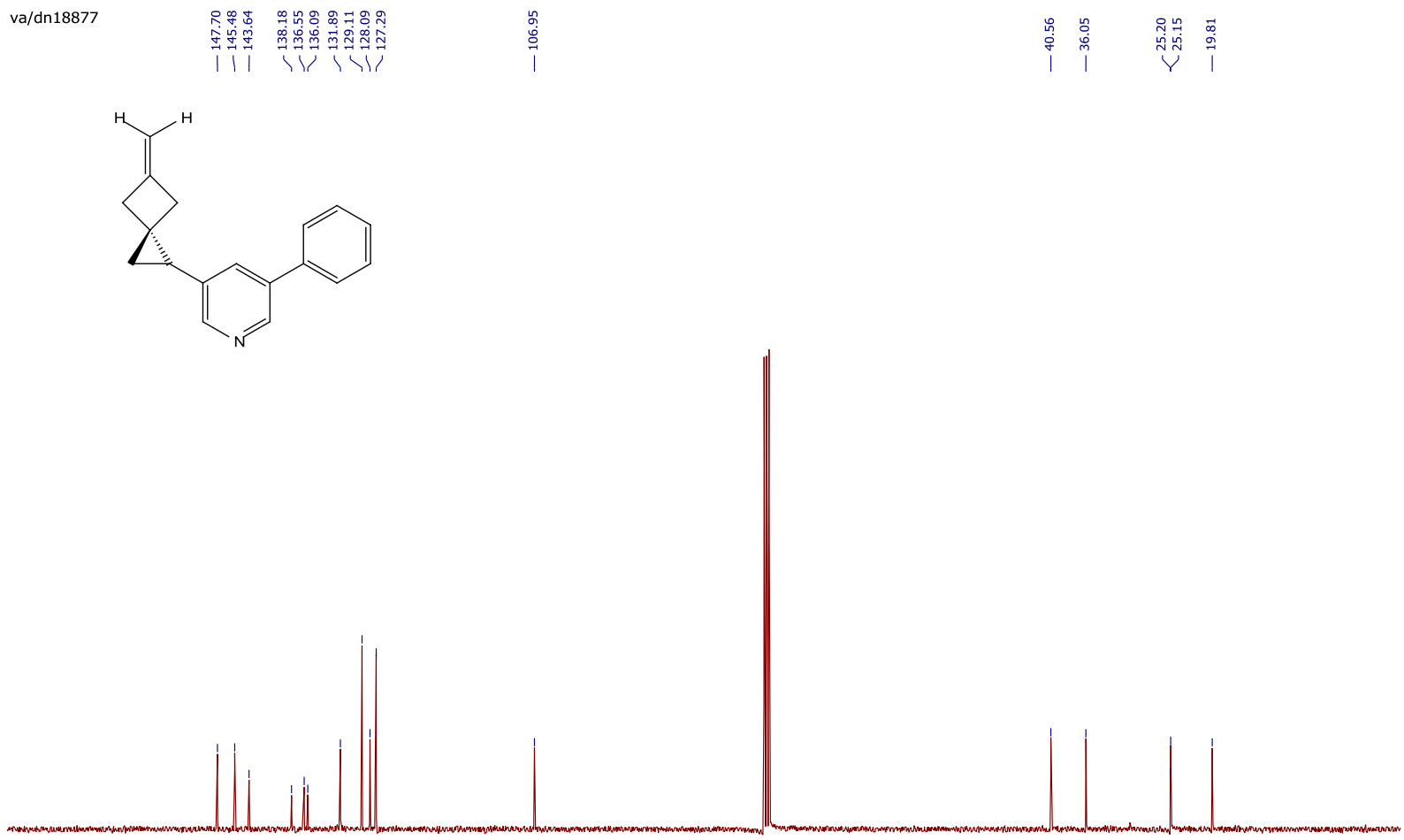

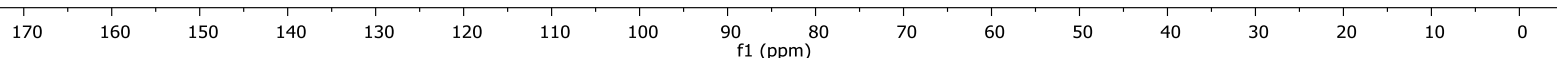




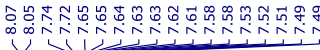
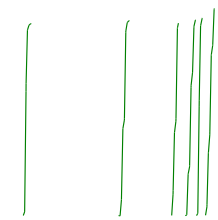

${ }^{1} \mathrm{H}$ NMR-7o

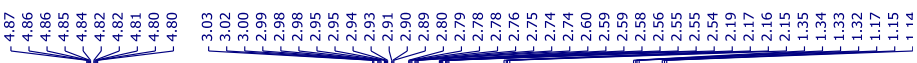
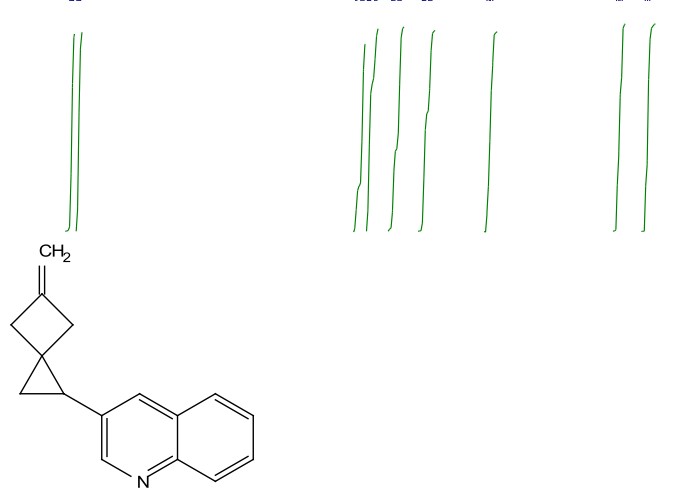
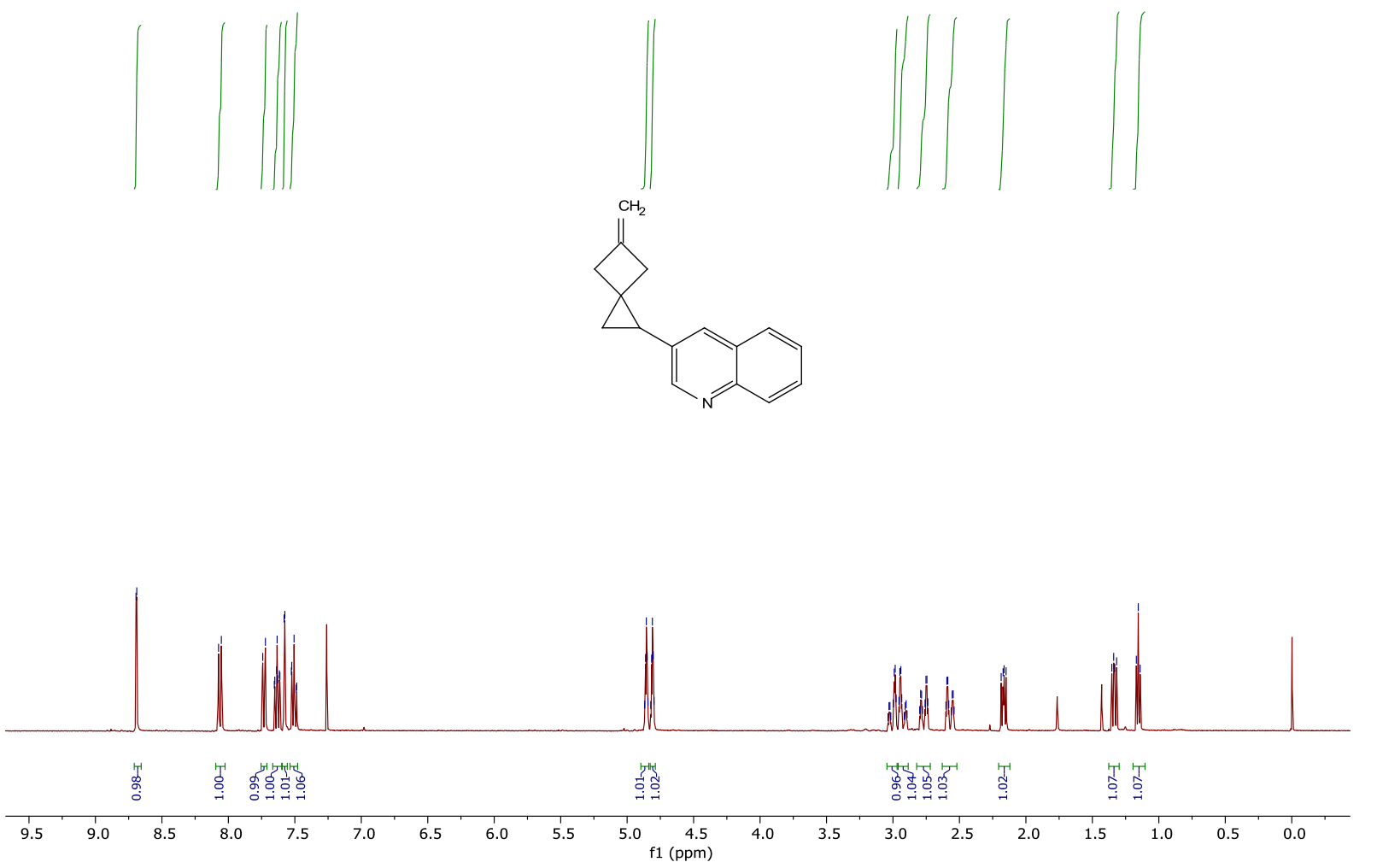

${ }^{13}$ C NMR-7o

va/dn18877 yu396 苟 | ।
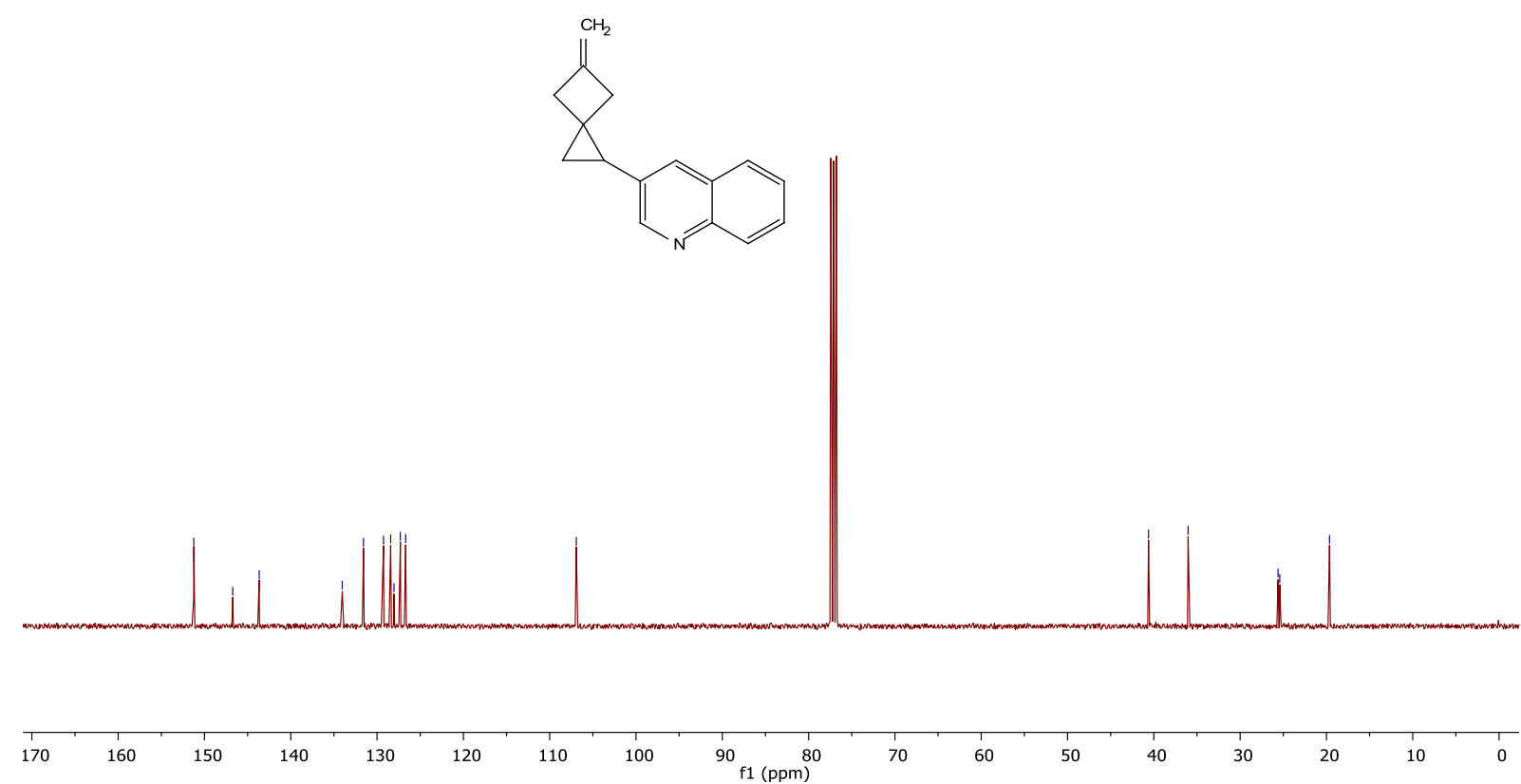
${ }^{1} \mathrm{H}$ NMR-7p

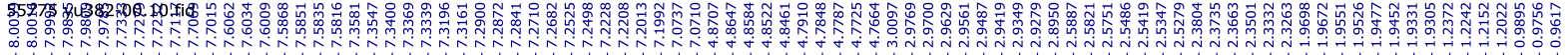

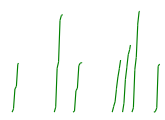
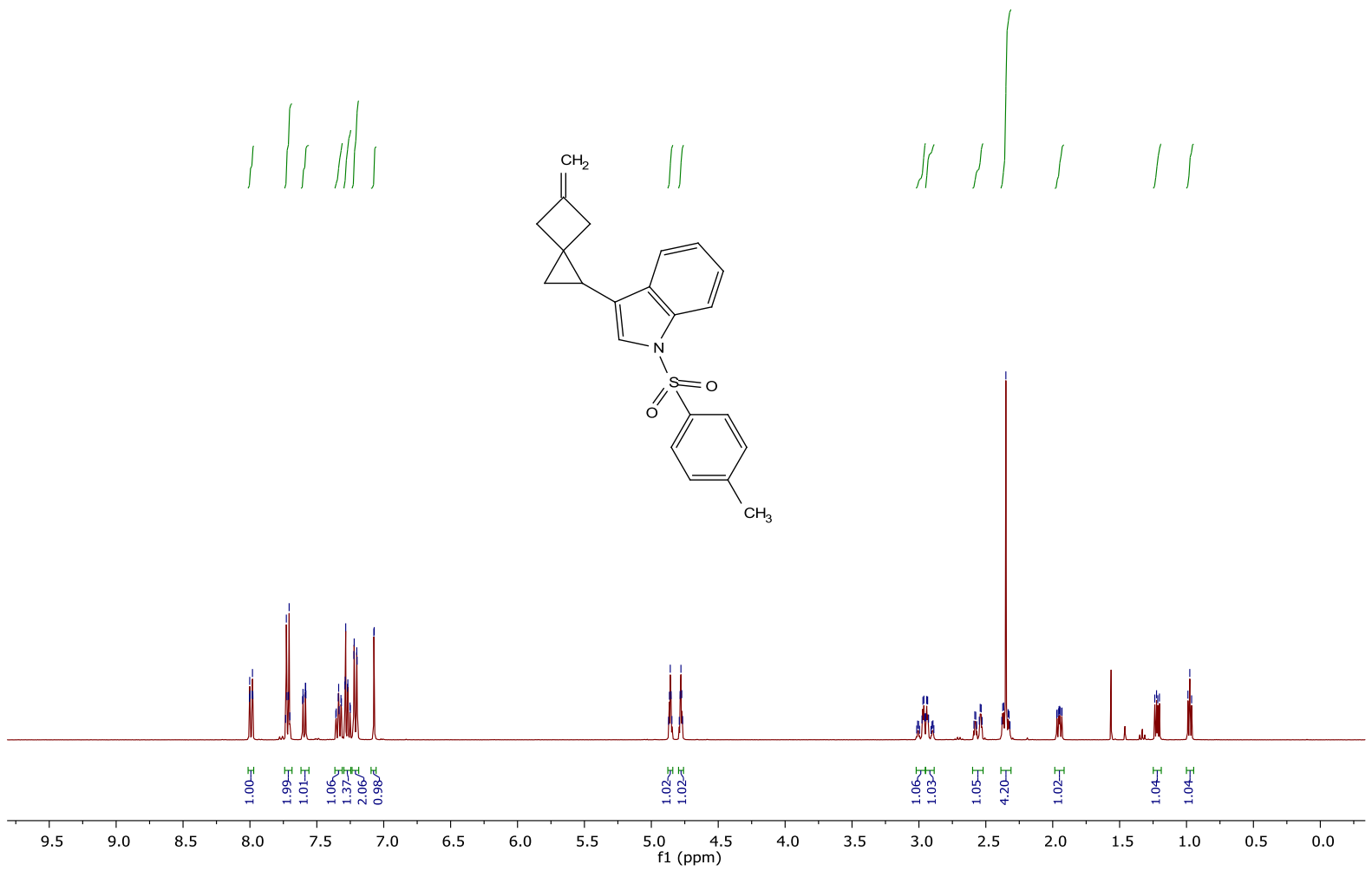

${ }^{13}$ C NMR-7p

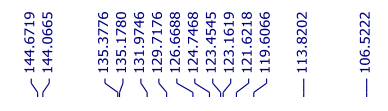

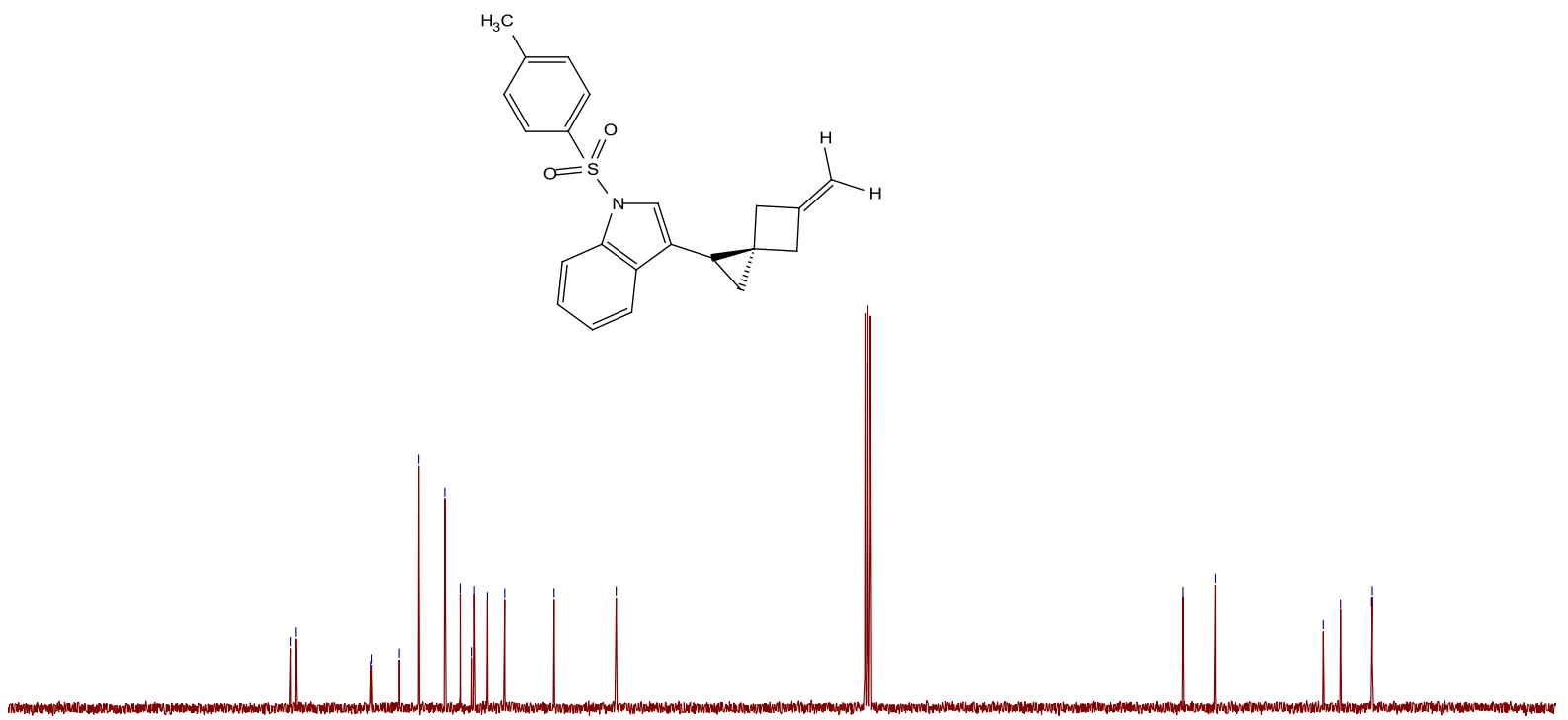


${ }^{1} \mathrm{H}$ NMR-7q

valdn18877 yu345
single_pulse
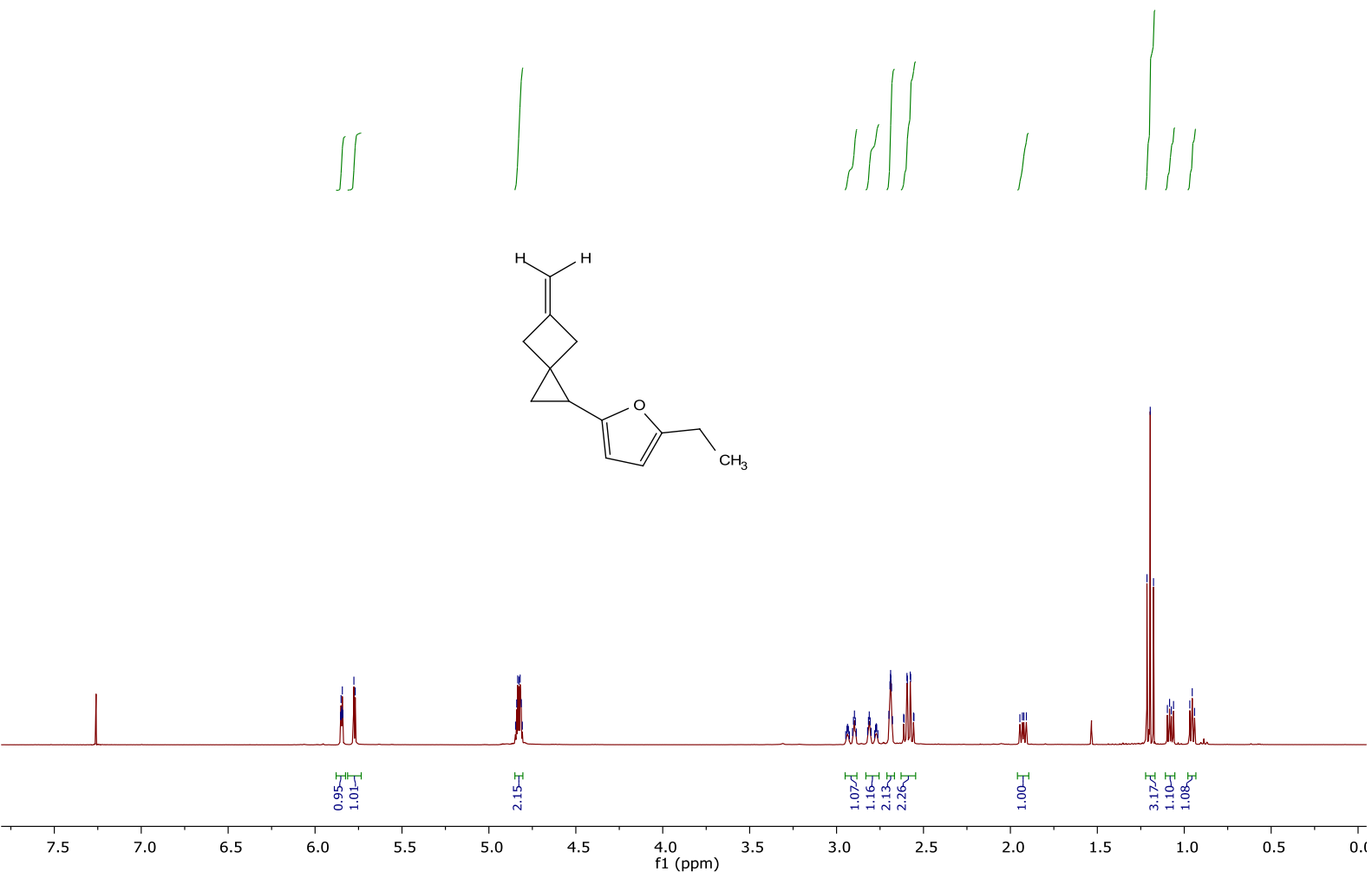

va/dn18877 yu345 |

${ }^{13}$ C NMR-7q
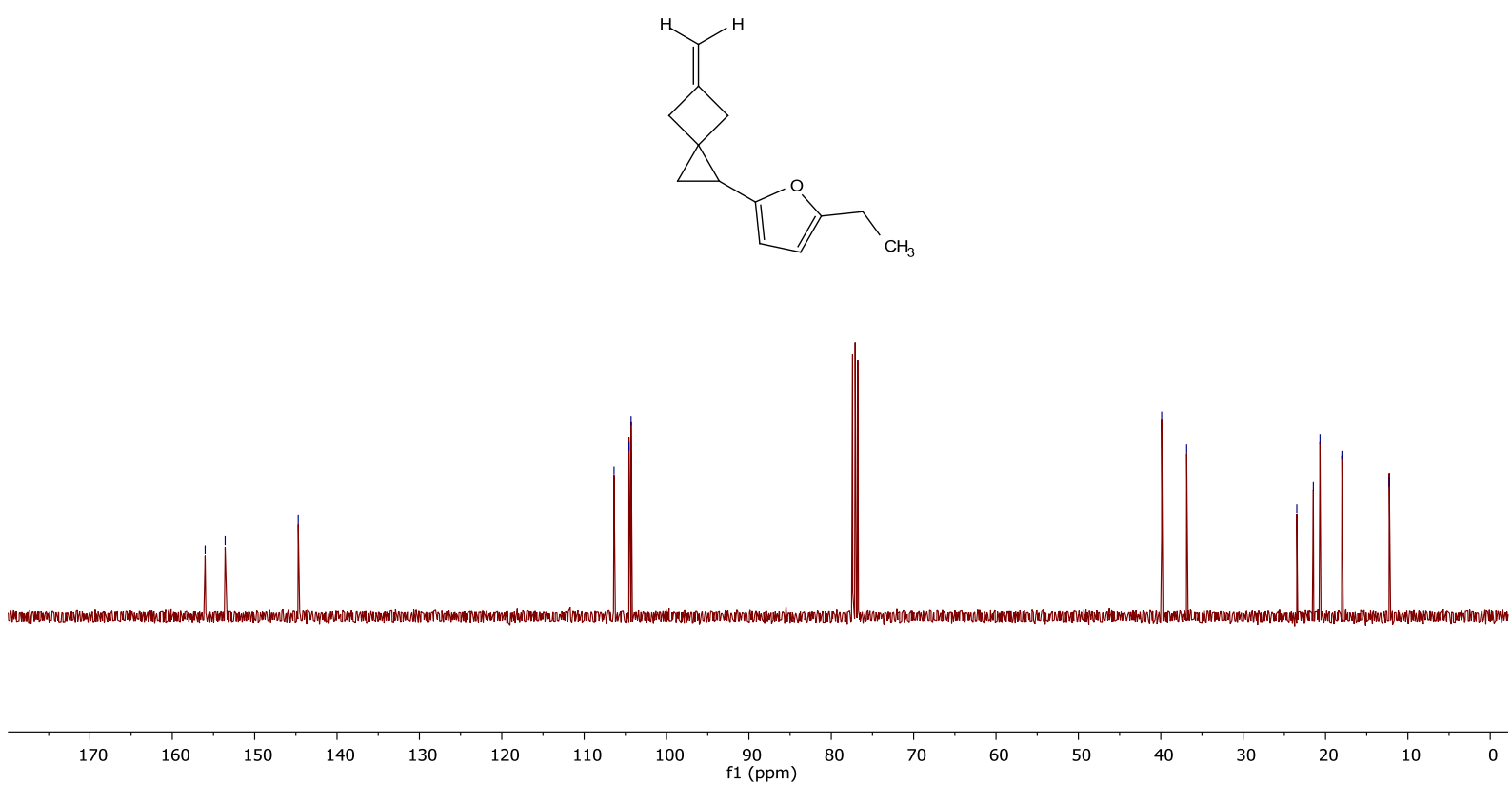
${ }^{1} \mathrm{H}$ NMR-7r

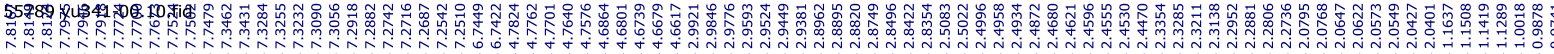
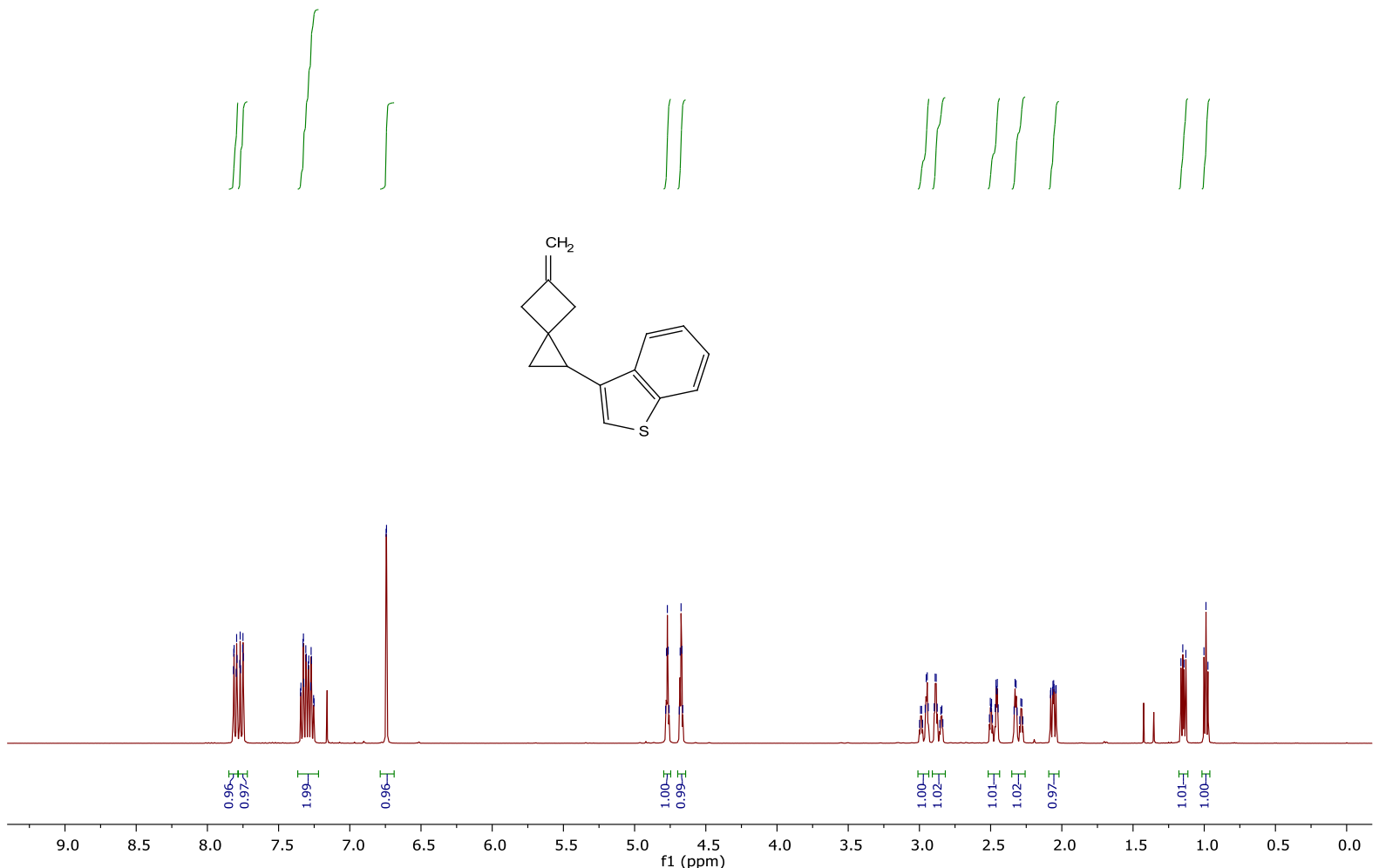

43103 yu341.11.fid

${ }^{13} \mathrm{C}$ NMR-7r

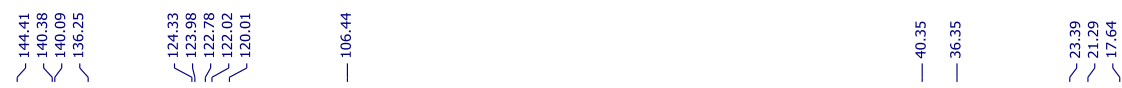

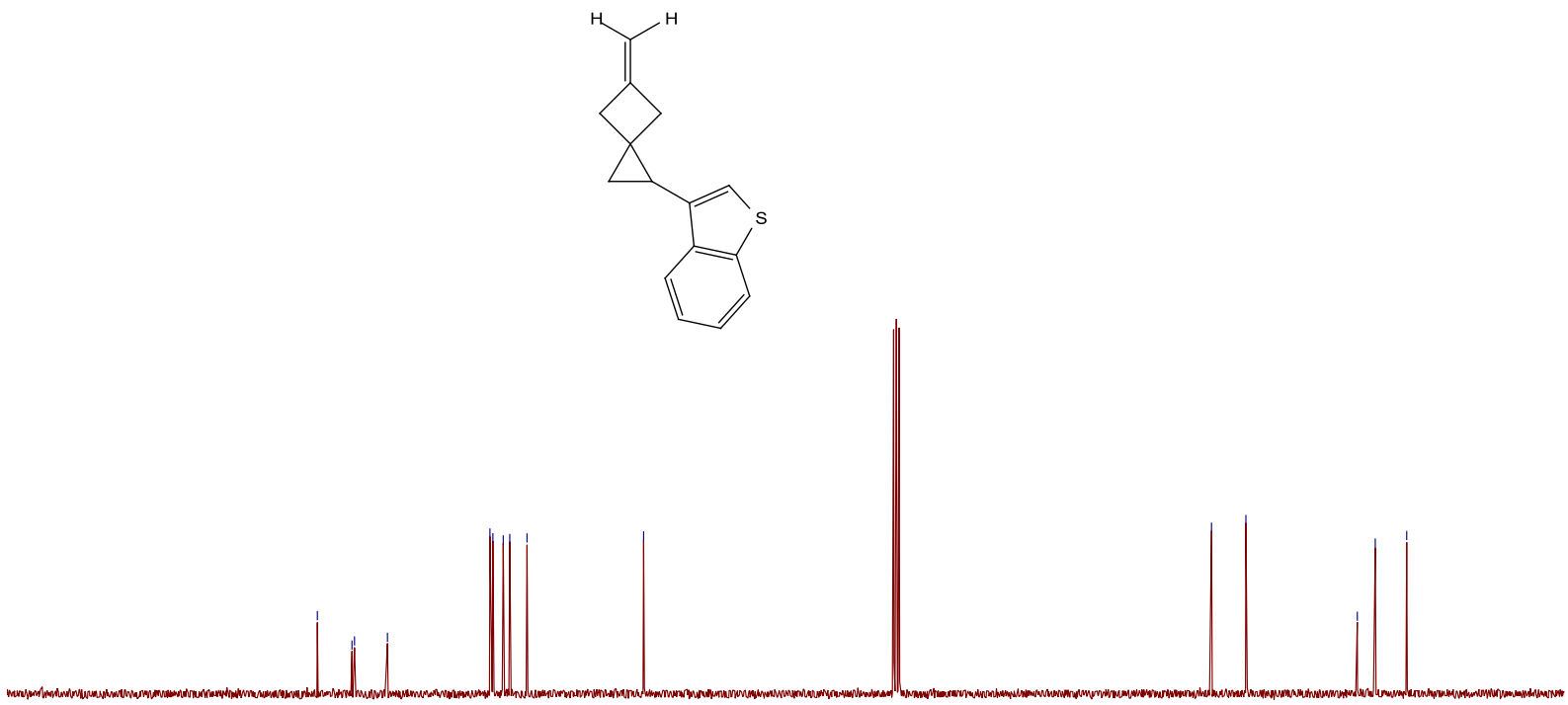

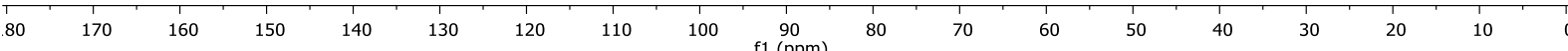


${ }^{1} \mathrm{H}$ NMR-7s

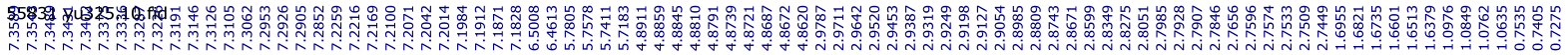
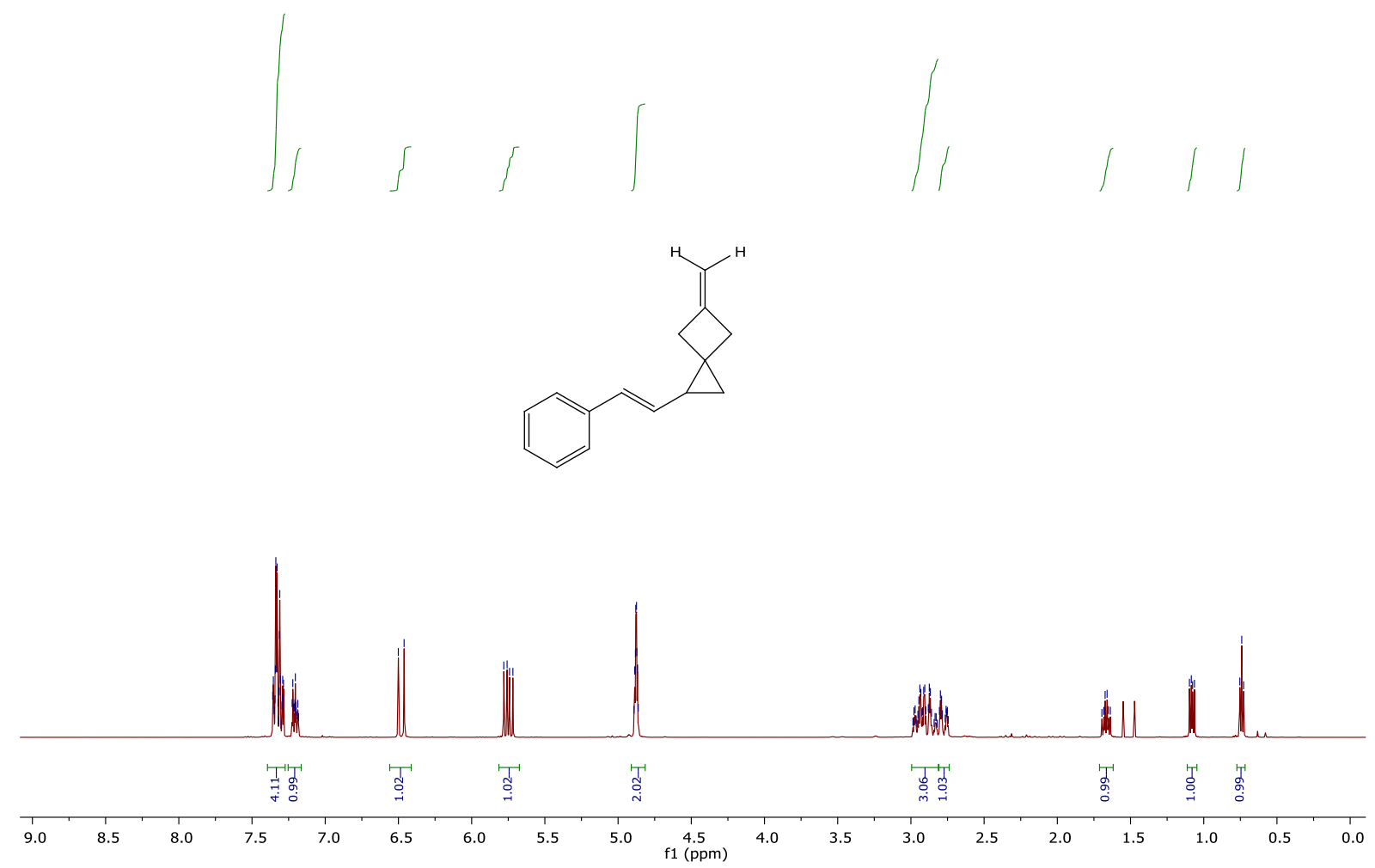

39859 yu278.12.fid

${ }^{13} \mathrm{C}$ NMR-7s
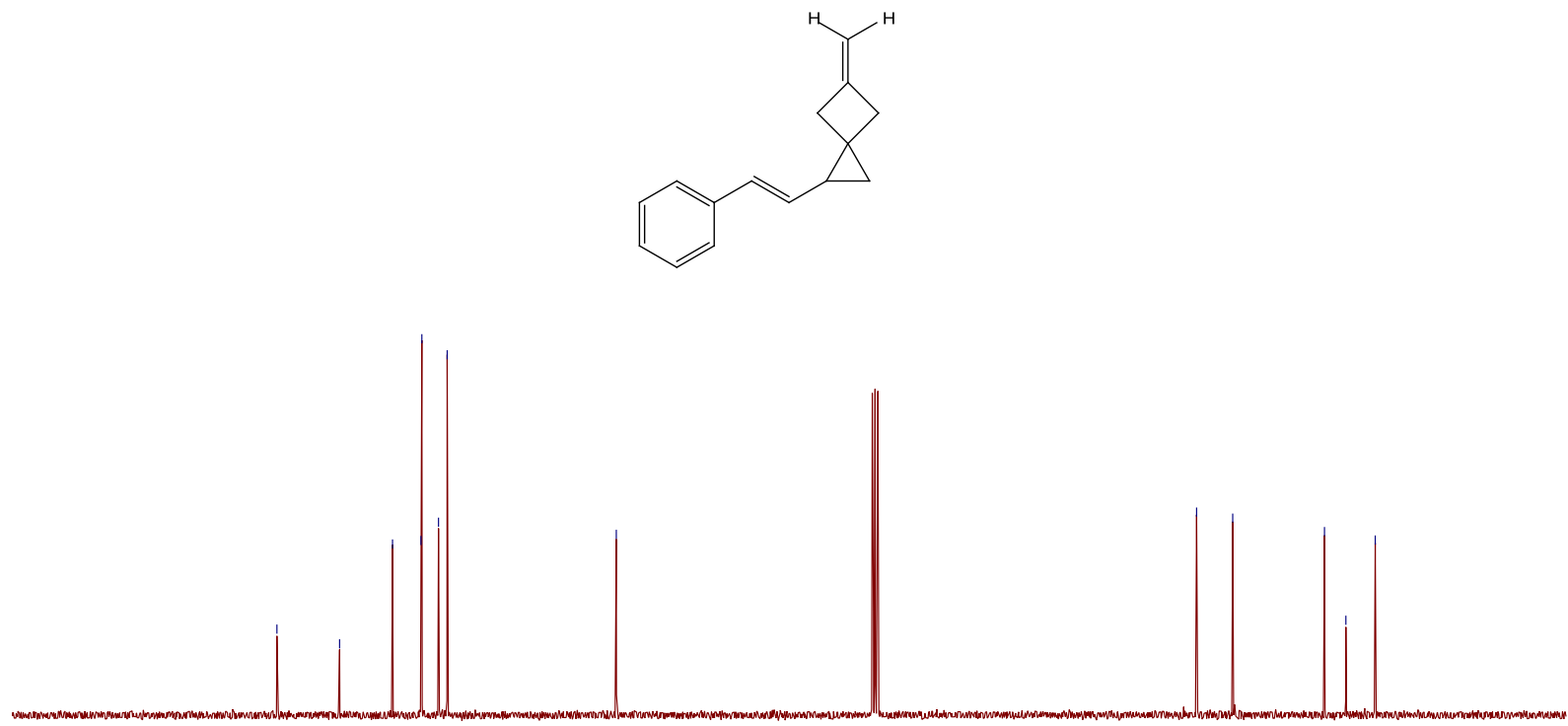

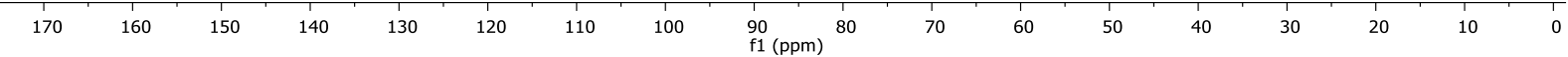


${ }^{1} \mathrm{H}$ NMR-7t

44018 yu333.10.fid

年是

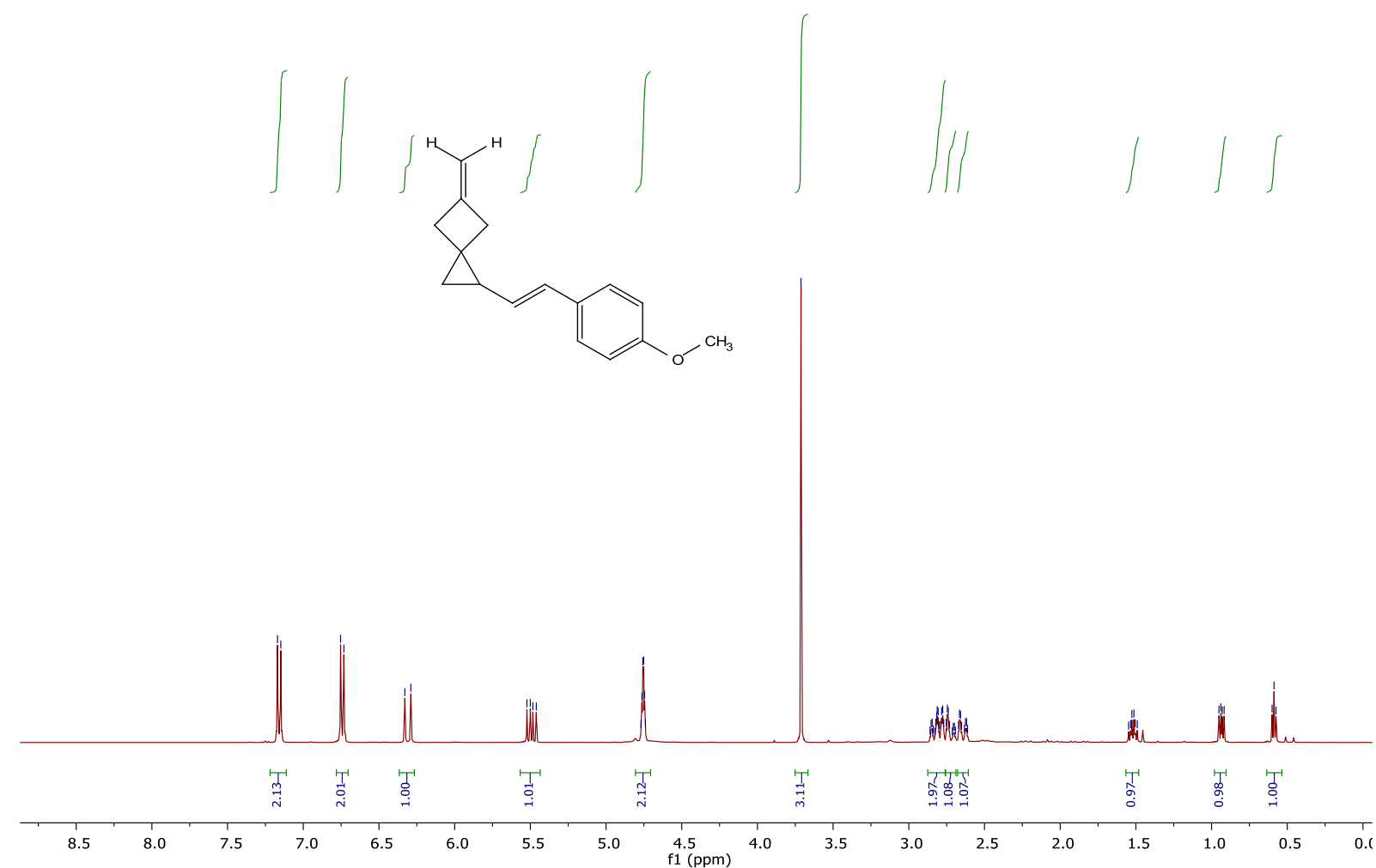

${ }^{13} \mathrm{C}$ NMR-7t

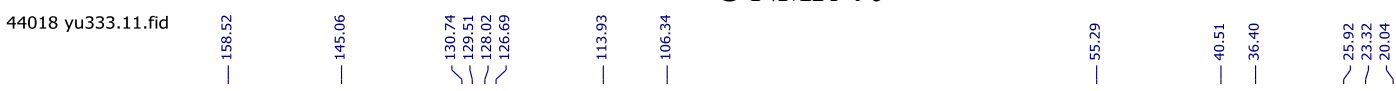
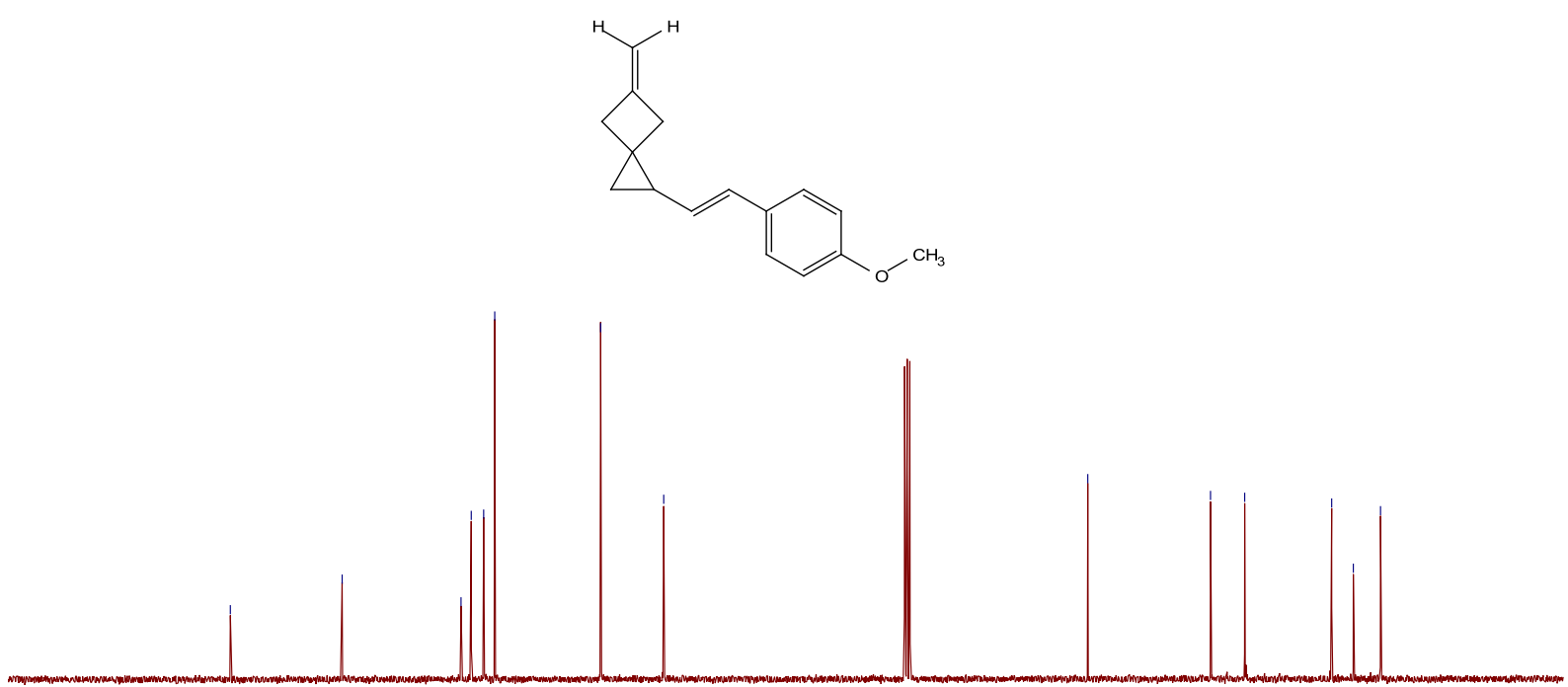

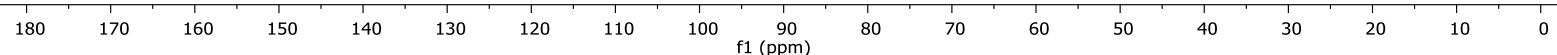


${ }^{1} \mathrm{H}$ NMR-7u

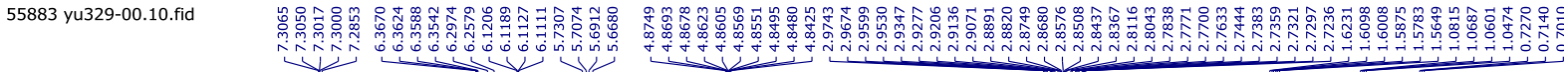
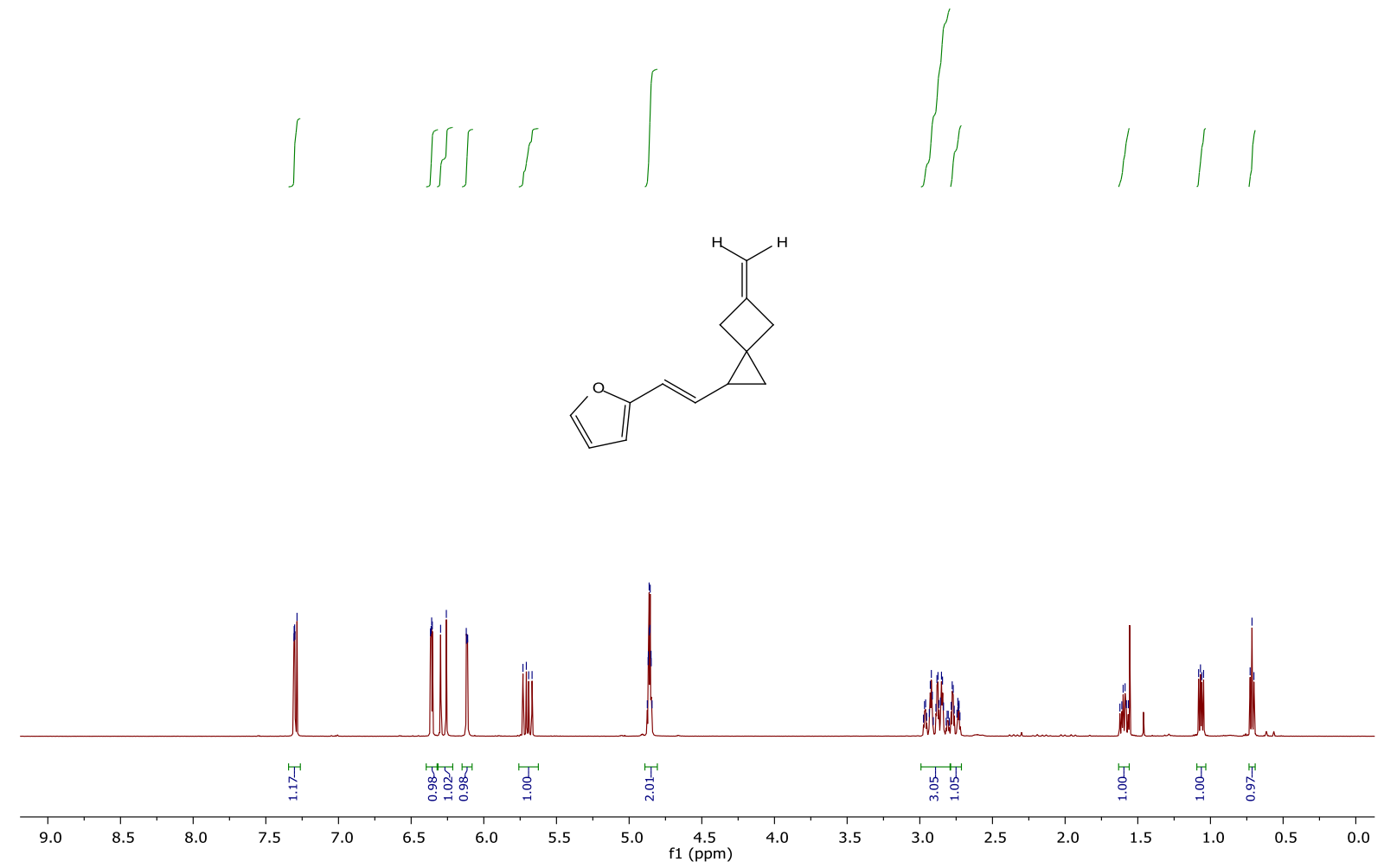

${ }^{13}$ C NMR-7u

42506 yu329.11.fid

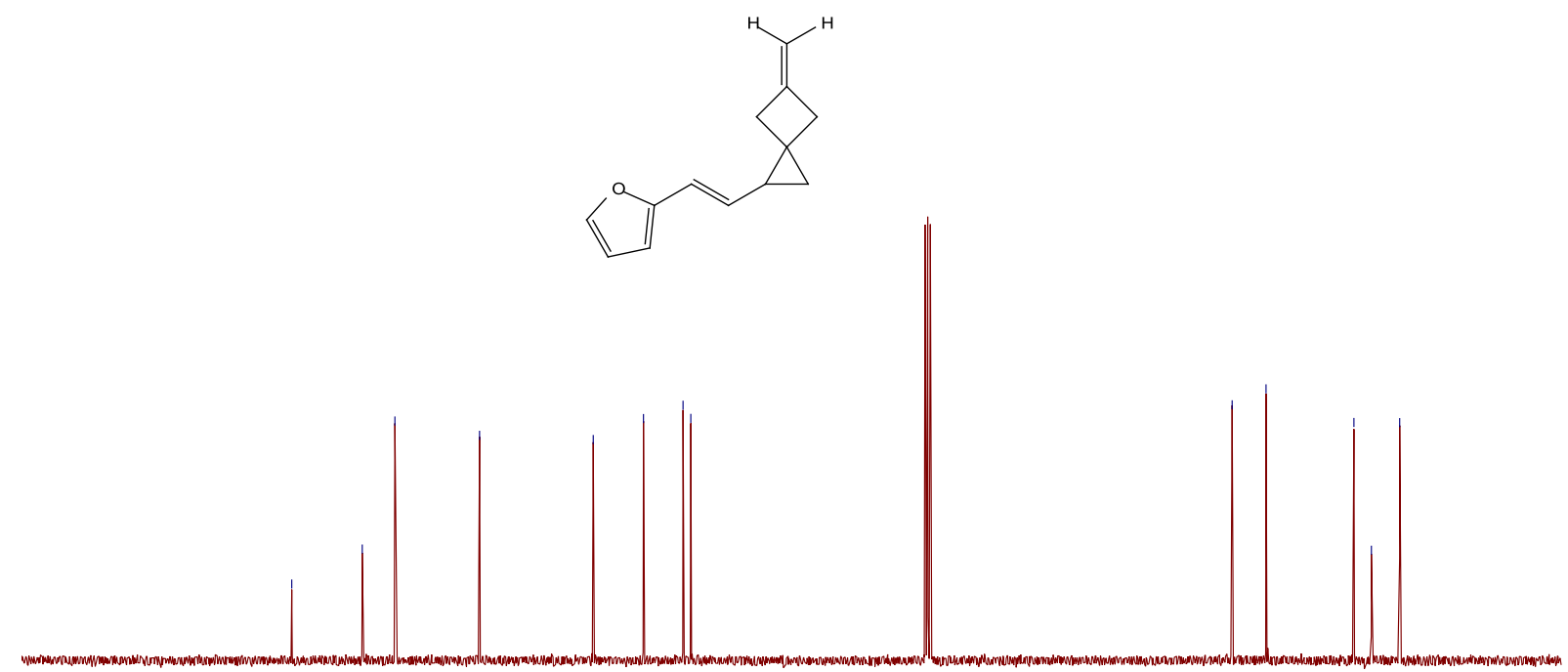

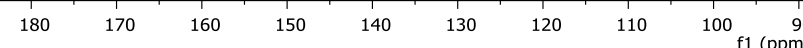


${ }^{1} \mathrm{H}$ NMR-7v

42705 yu330.10.fid

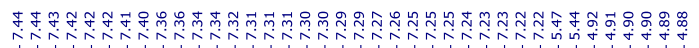

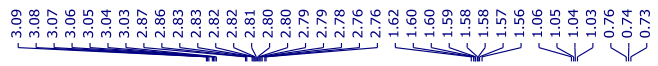
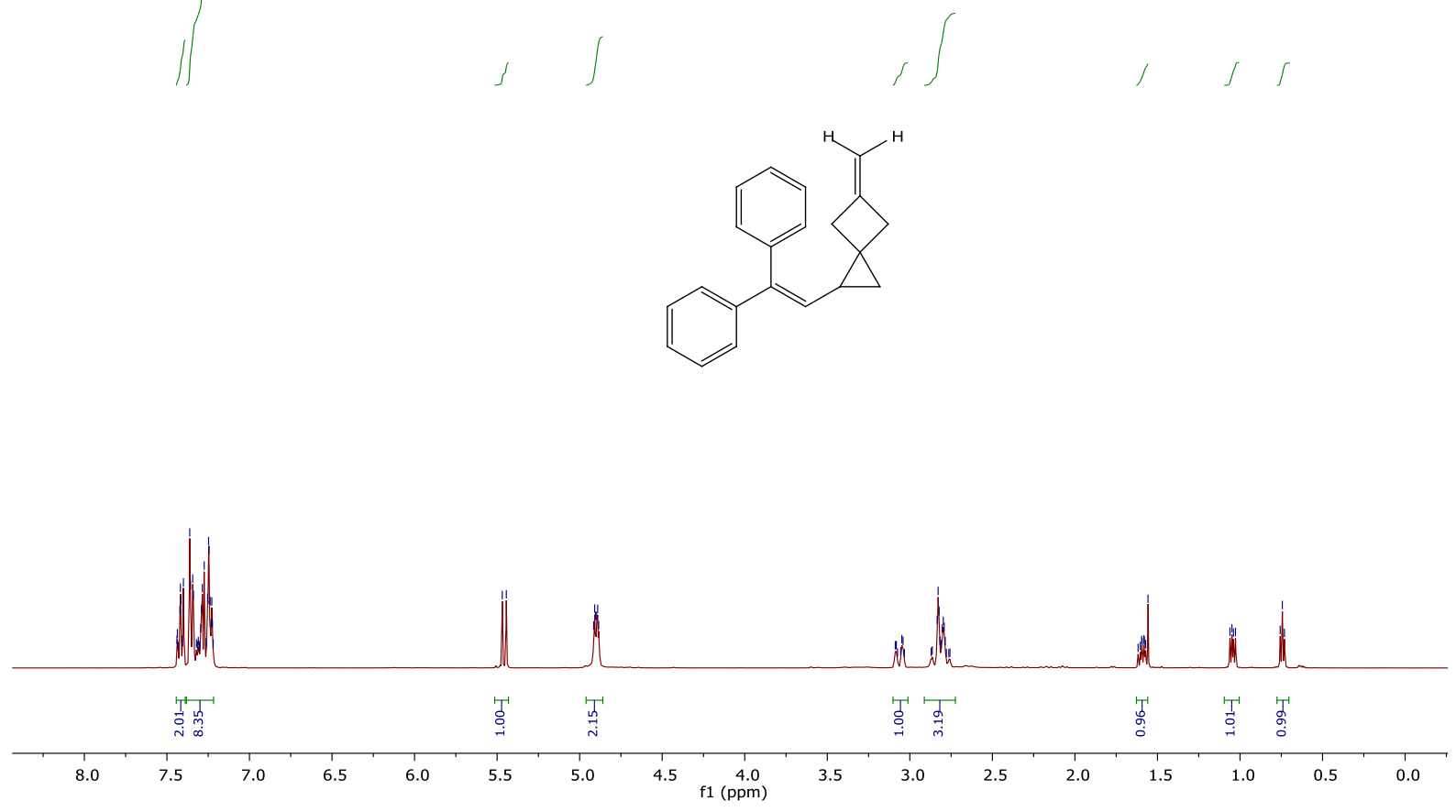

${ }^{13} \mathrm{C}$ NMR-7v

42705 yu330.11.fid

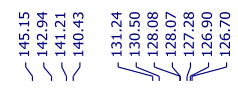

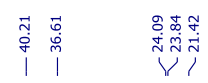
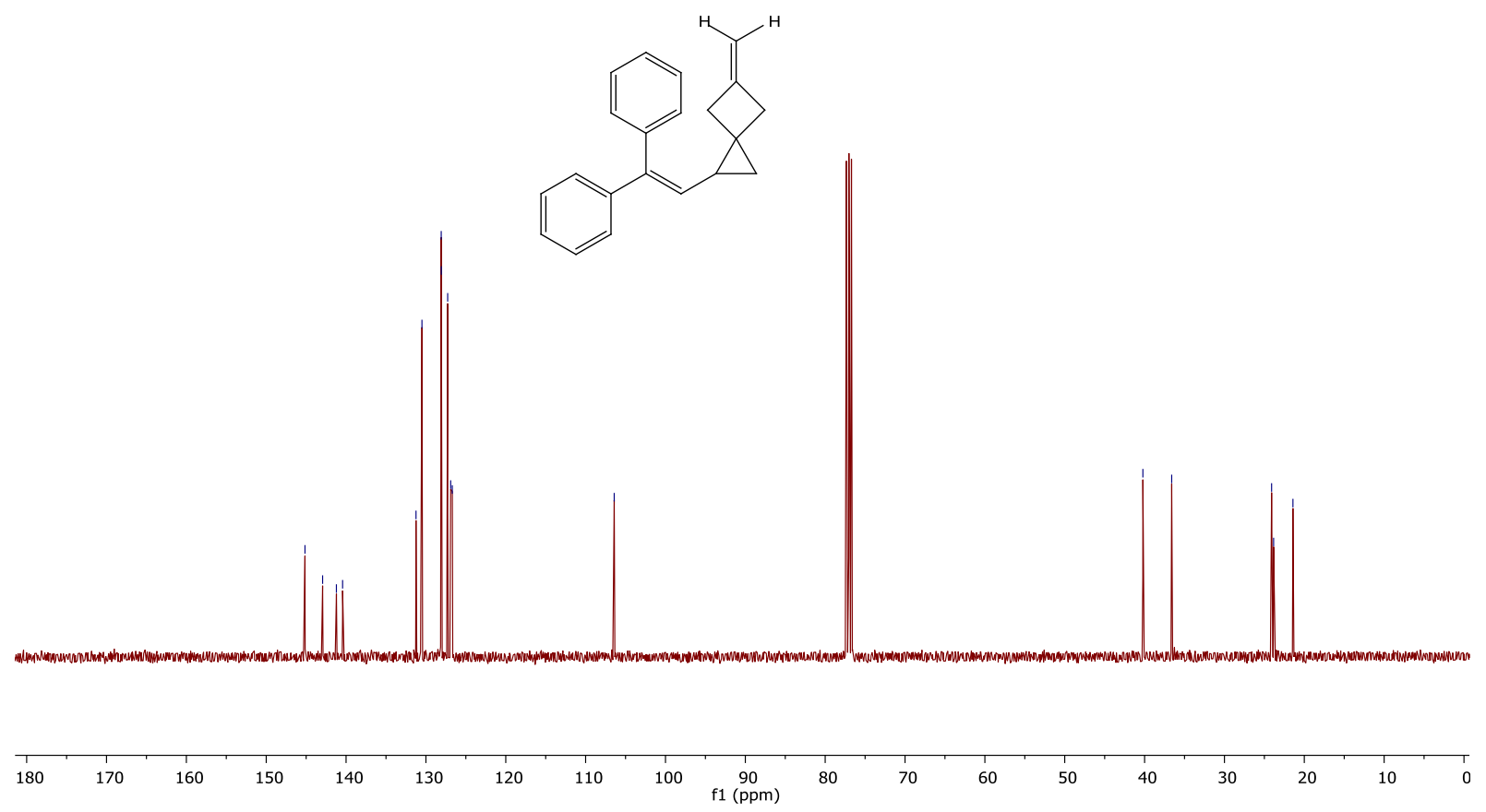

S87 


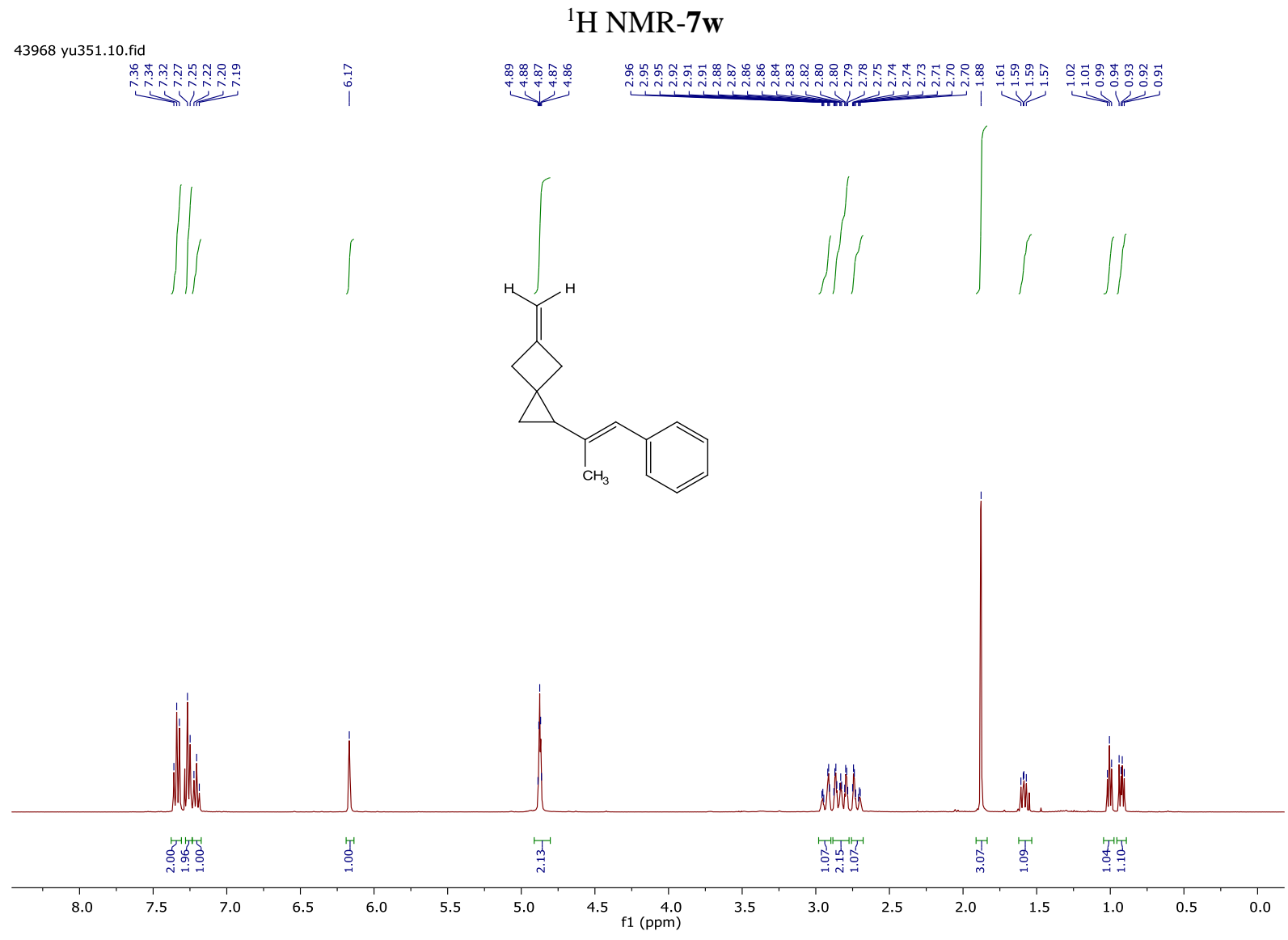

43968 yu351.11.fid

${ }^{13}$ C NMR-7w

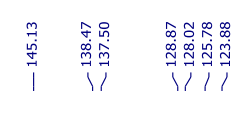
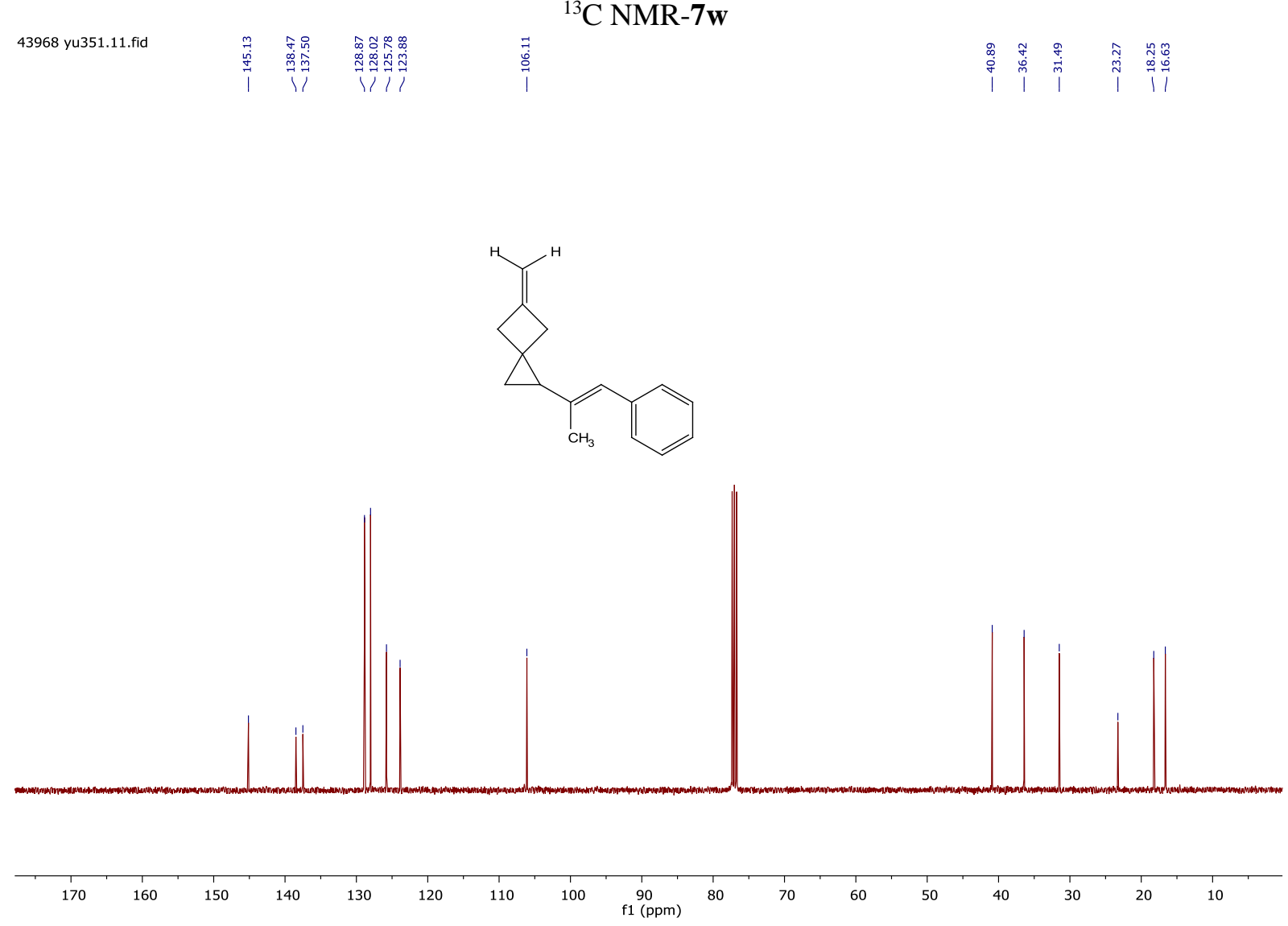

S88 


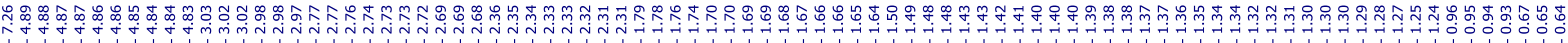

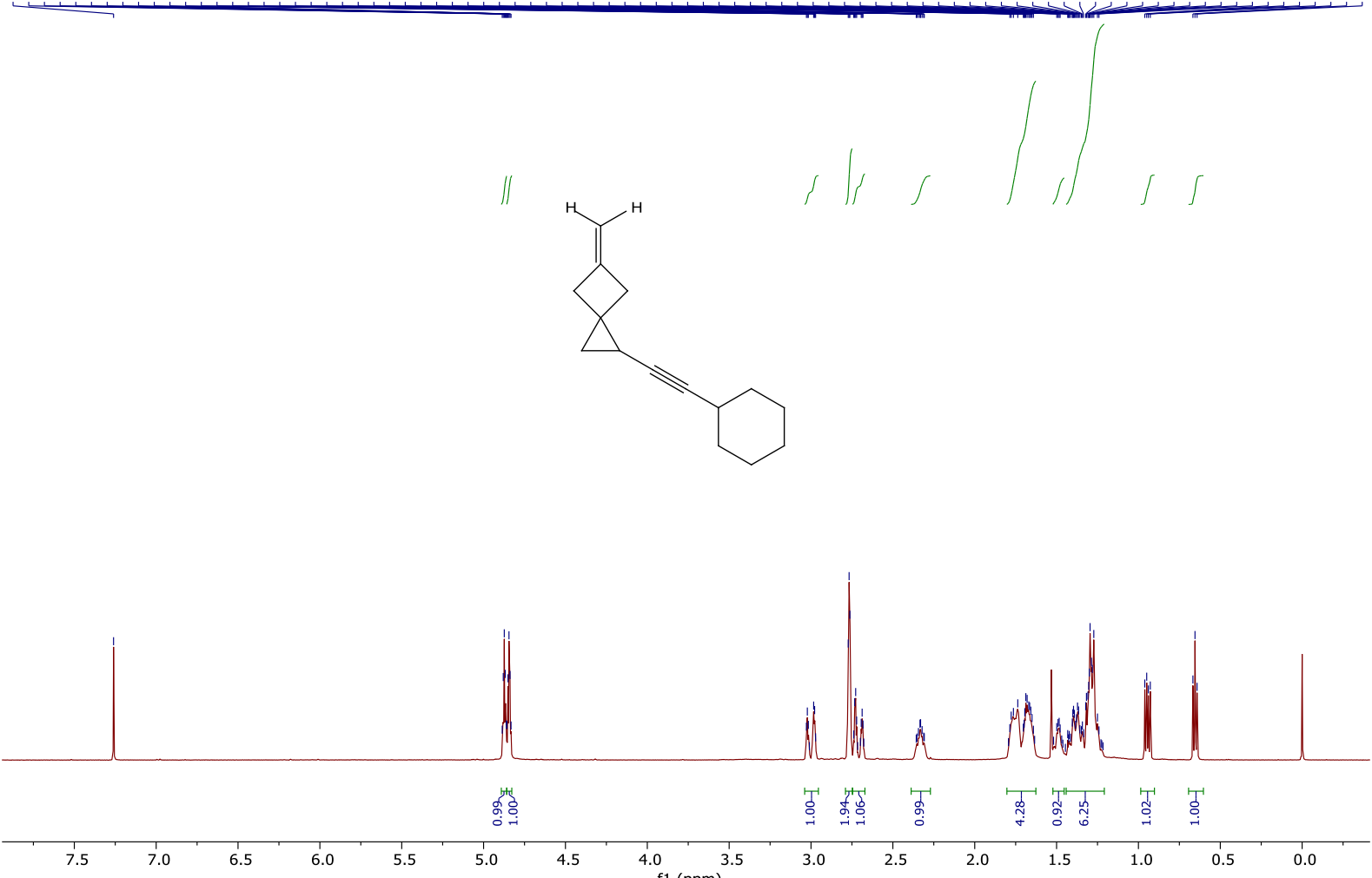

4594 yu377.11.fid

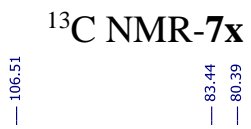

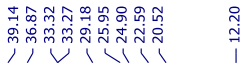
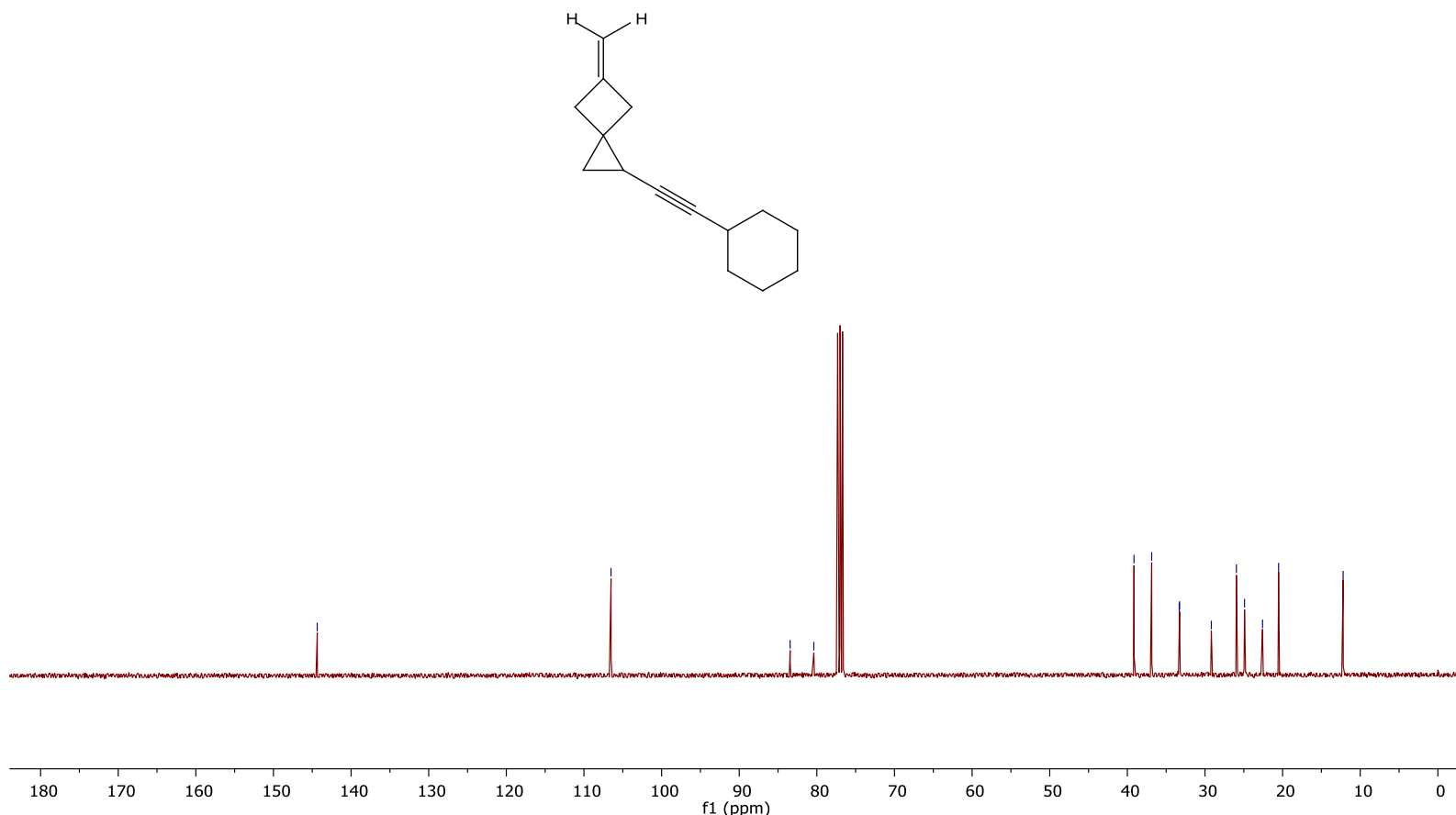

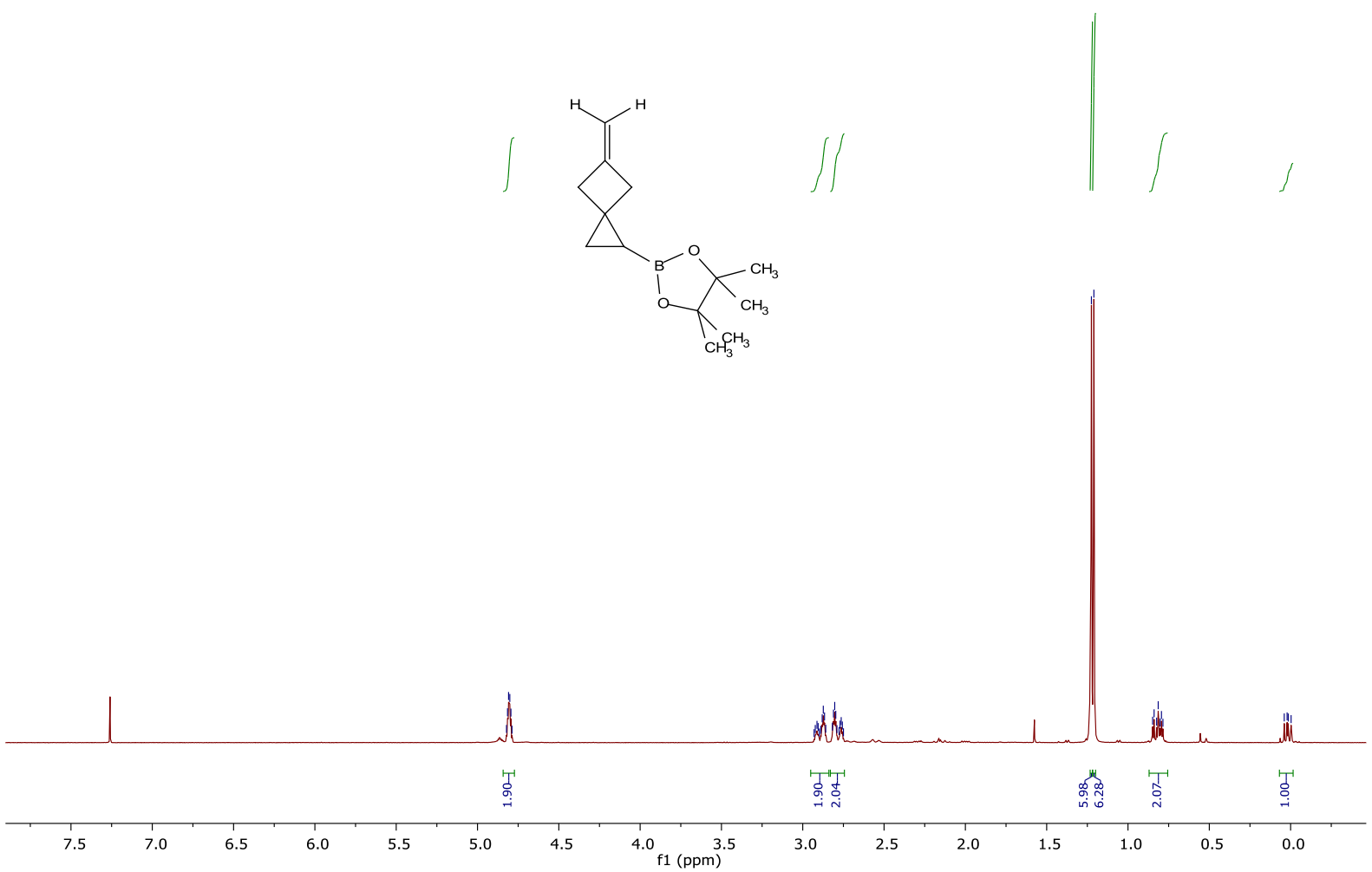

va/dn18877 yu375 1

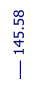

${ }^{13} \mathrm{C}$ NMR-12a

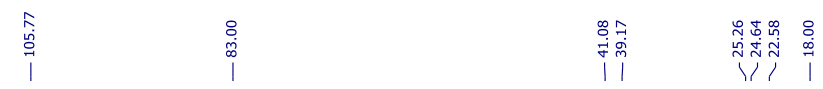

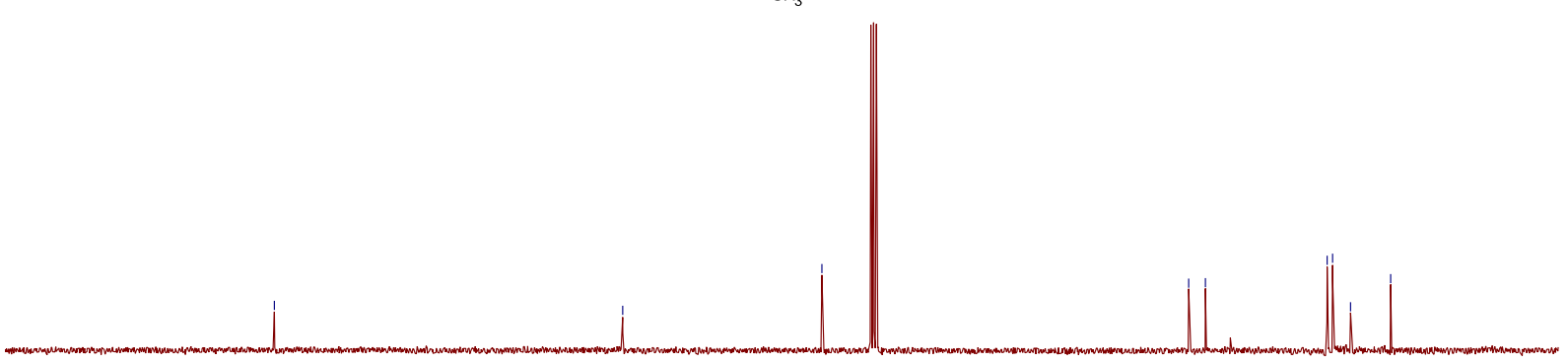

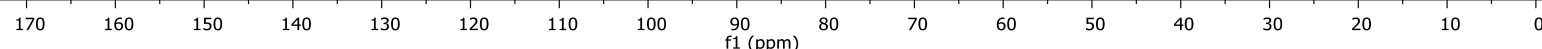


${ }^{1} \mathrm{H}$ NMR-trans 12b

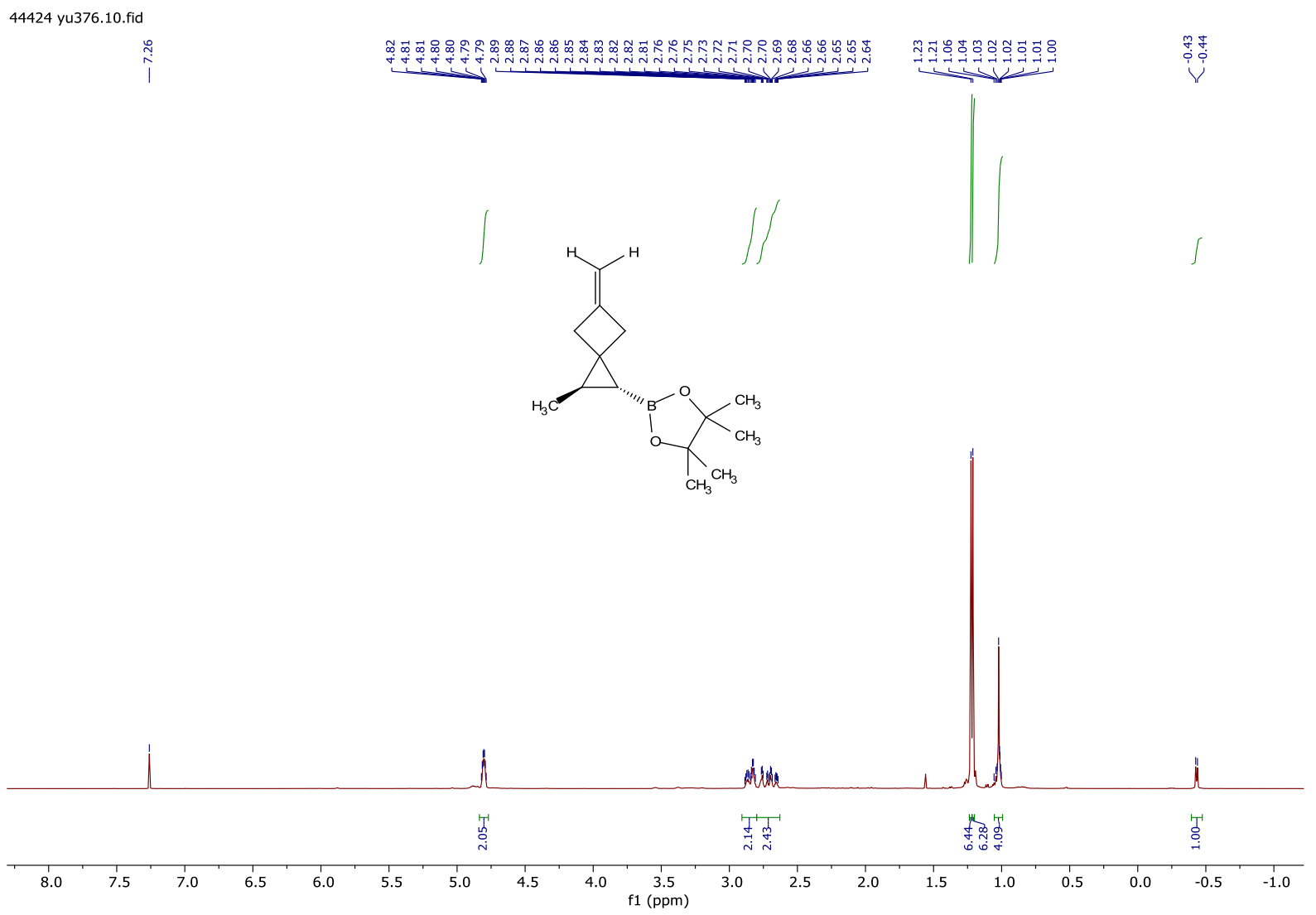

YU17336_YU376_NOESY1D01

\section{NOESY NMR-trans 12b}

YU17336_YU376_NOESY1D01

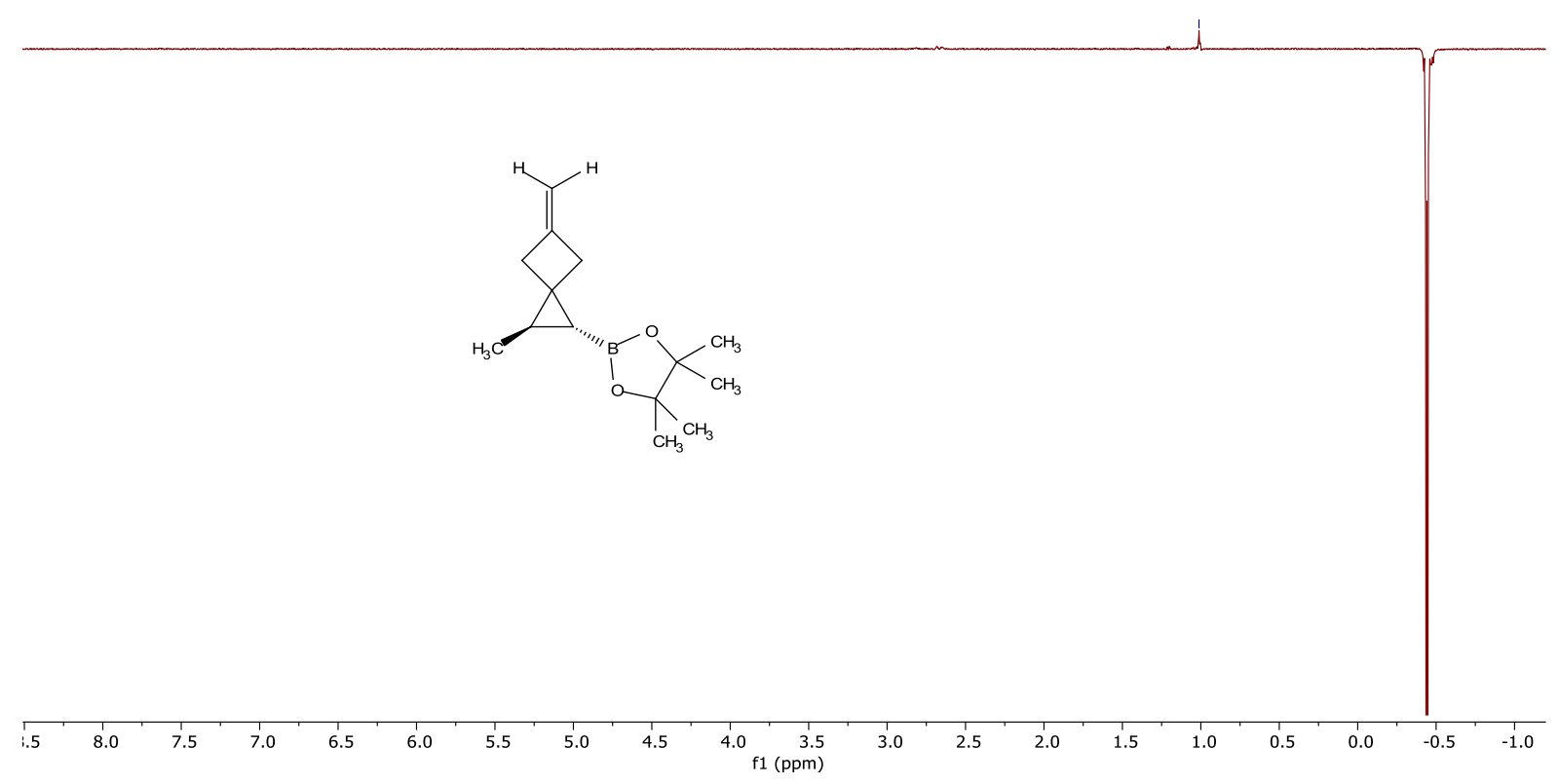



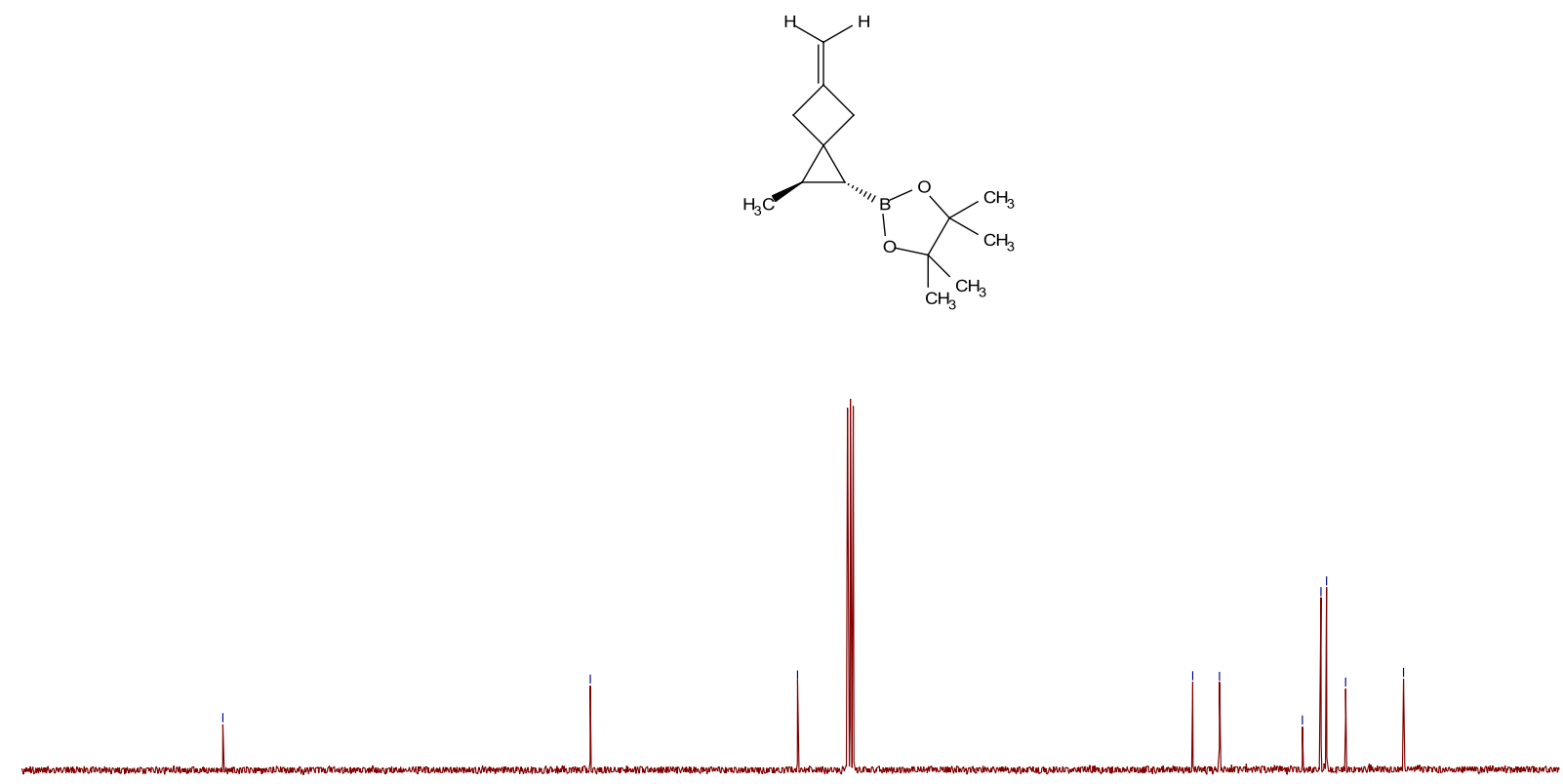

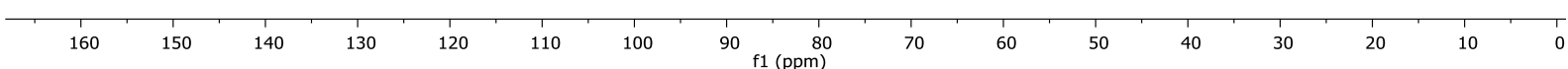

\section{${ }^{11} \mathrm{~B}$ NMR-trans 12b}

44424 yu376.15.fid
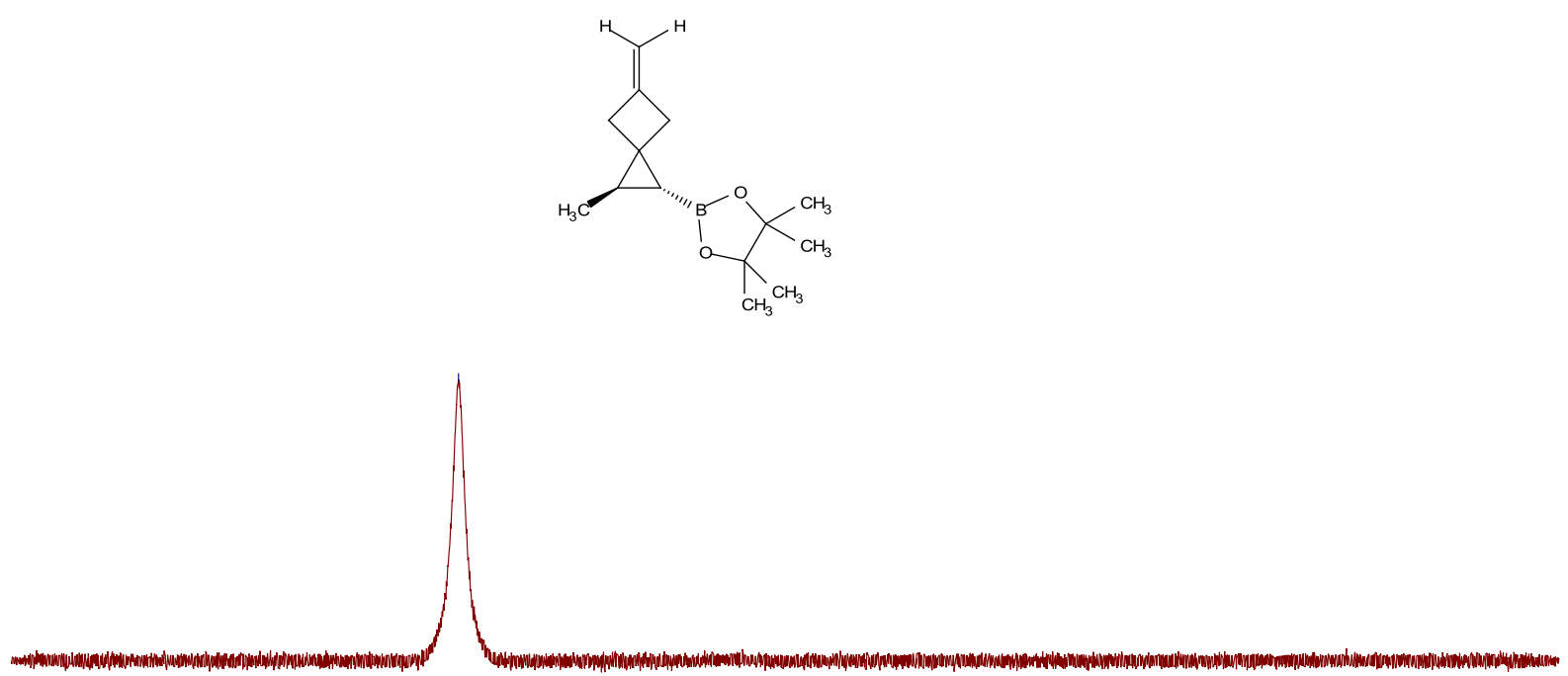

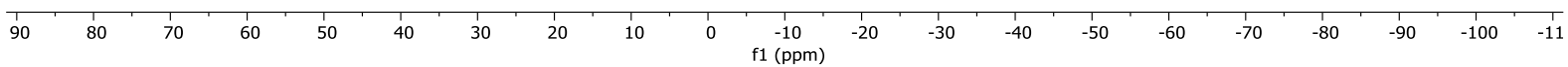



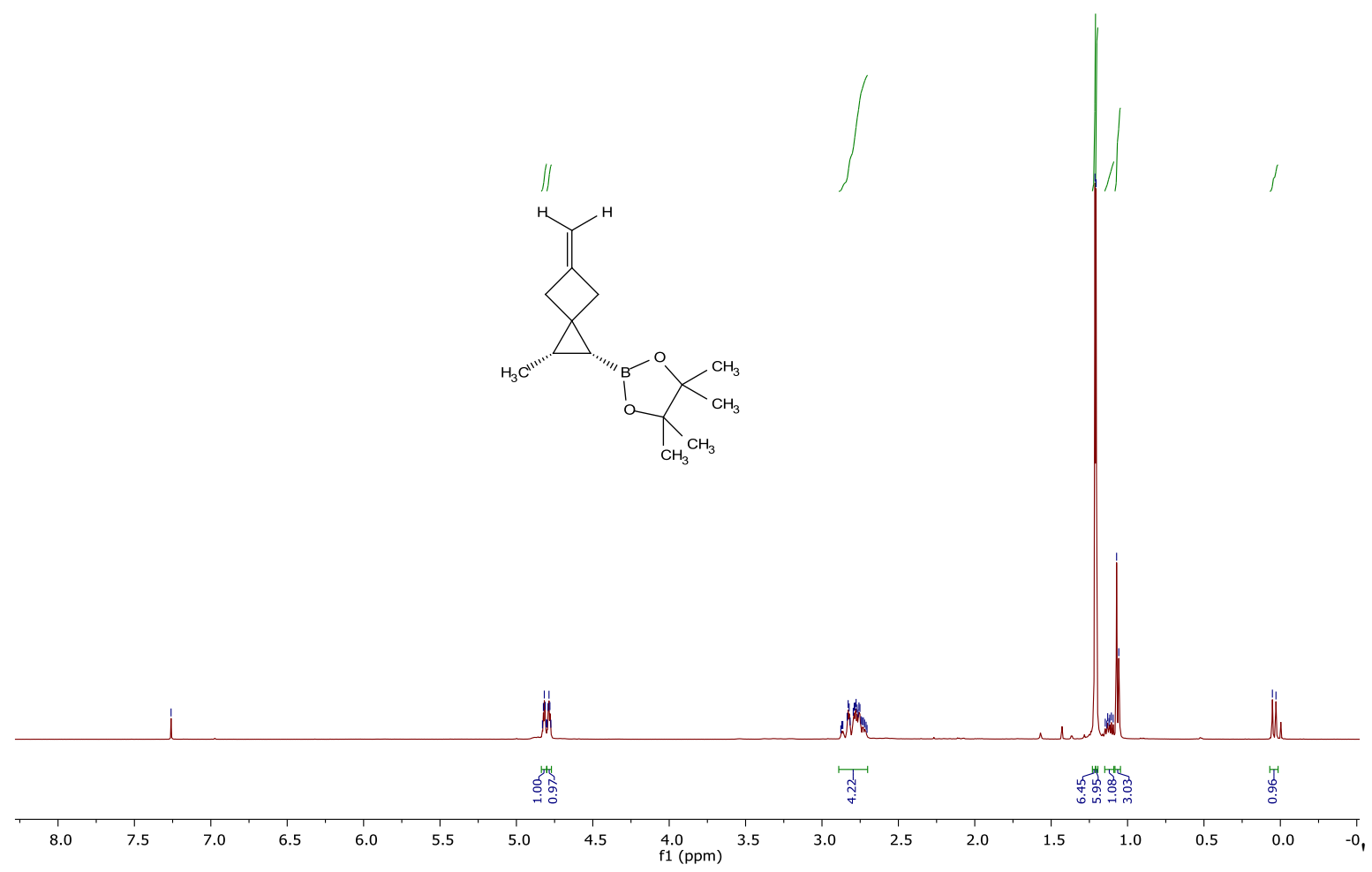

NOESY NMR-cis 12b

YU22614_YU401_NOESY1D_001

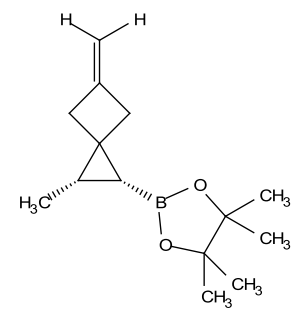



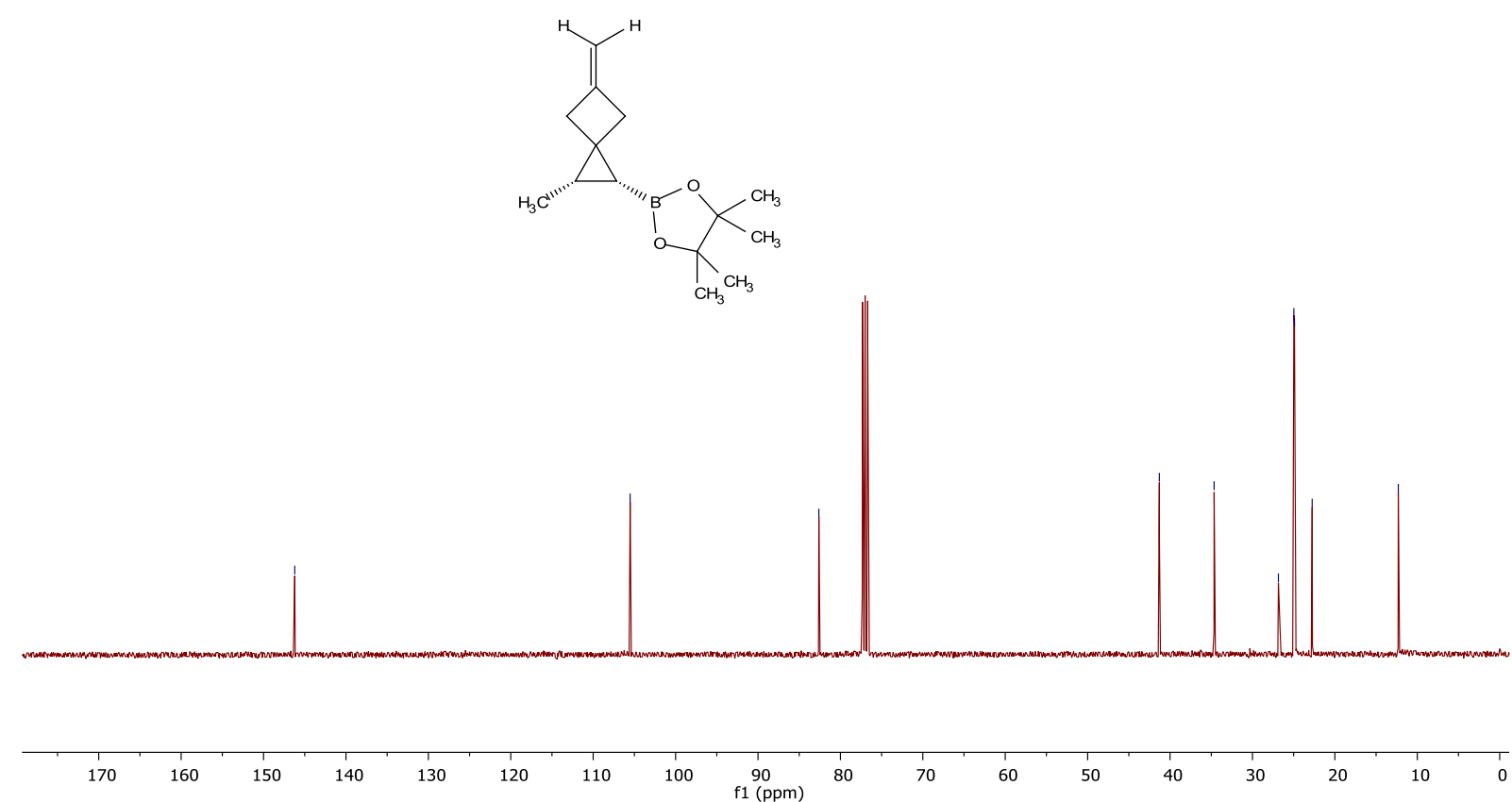

${ }^{11} \mathrm{~B}$ NMR-cis $\mathbf{1 2 b}$

45551 yu401.11.fid
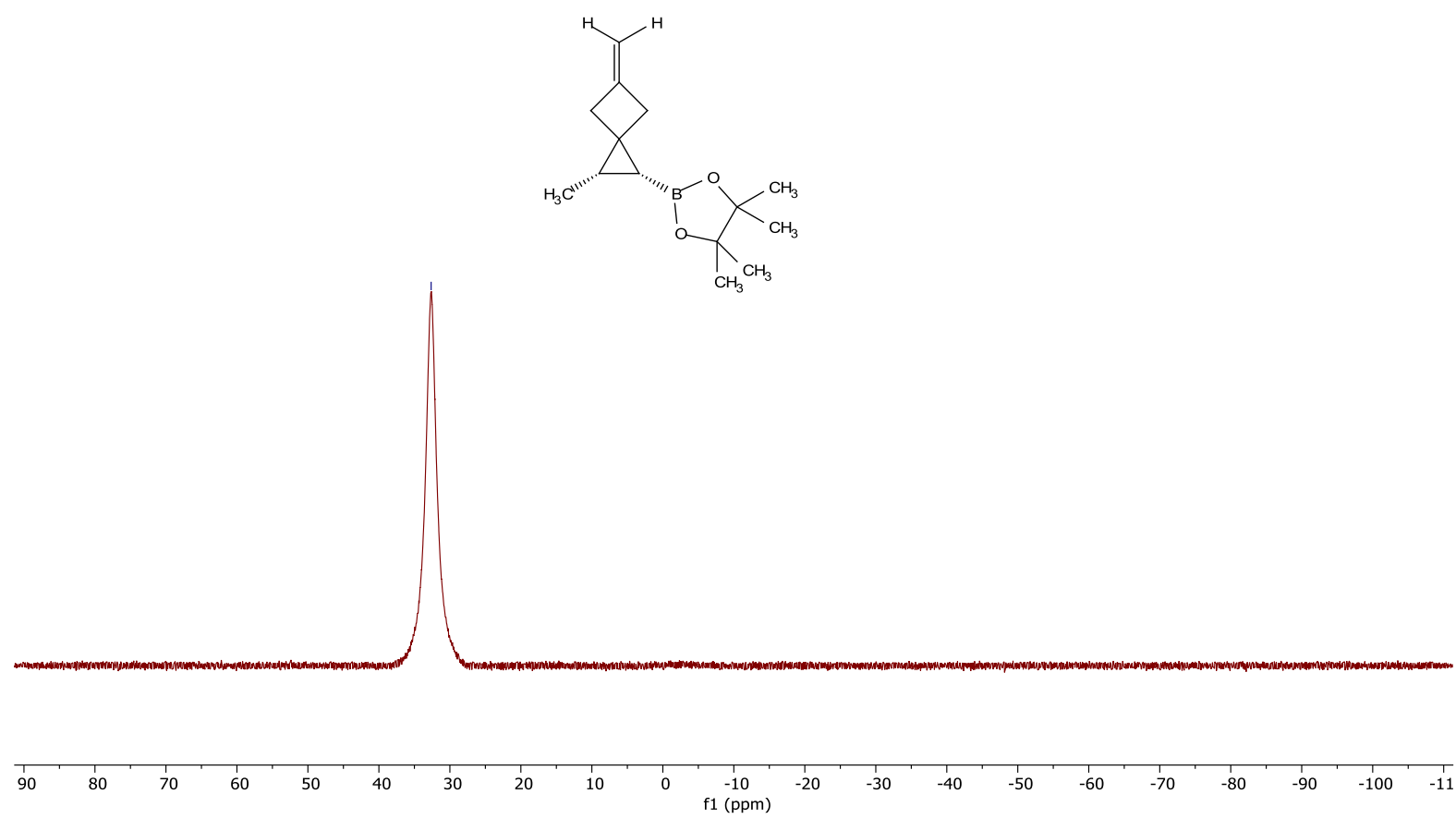


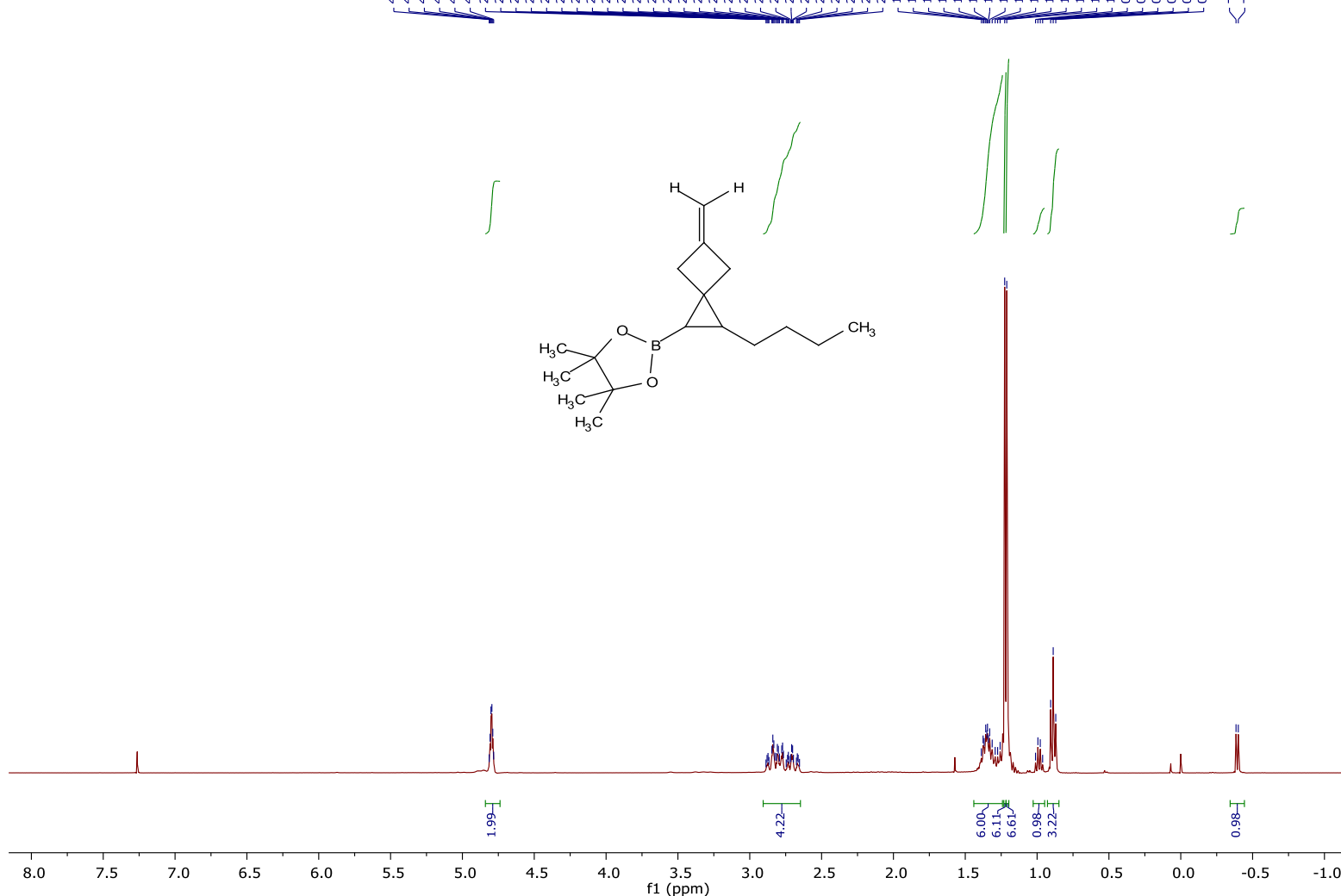

\section{${ }^{13} \mathrm{C}$ NMR-12c}

44745 yu381.12.fid
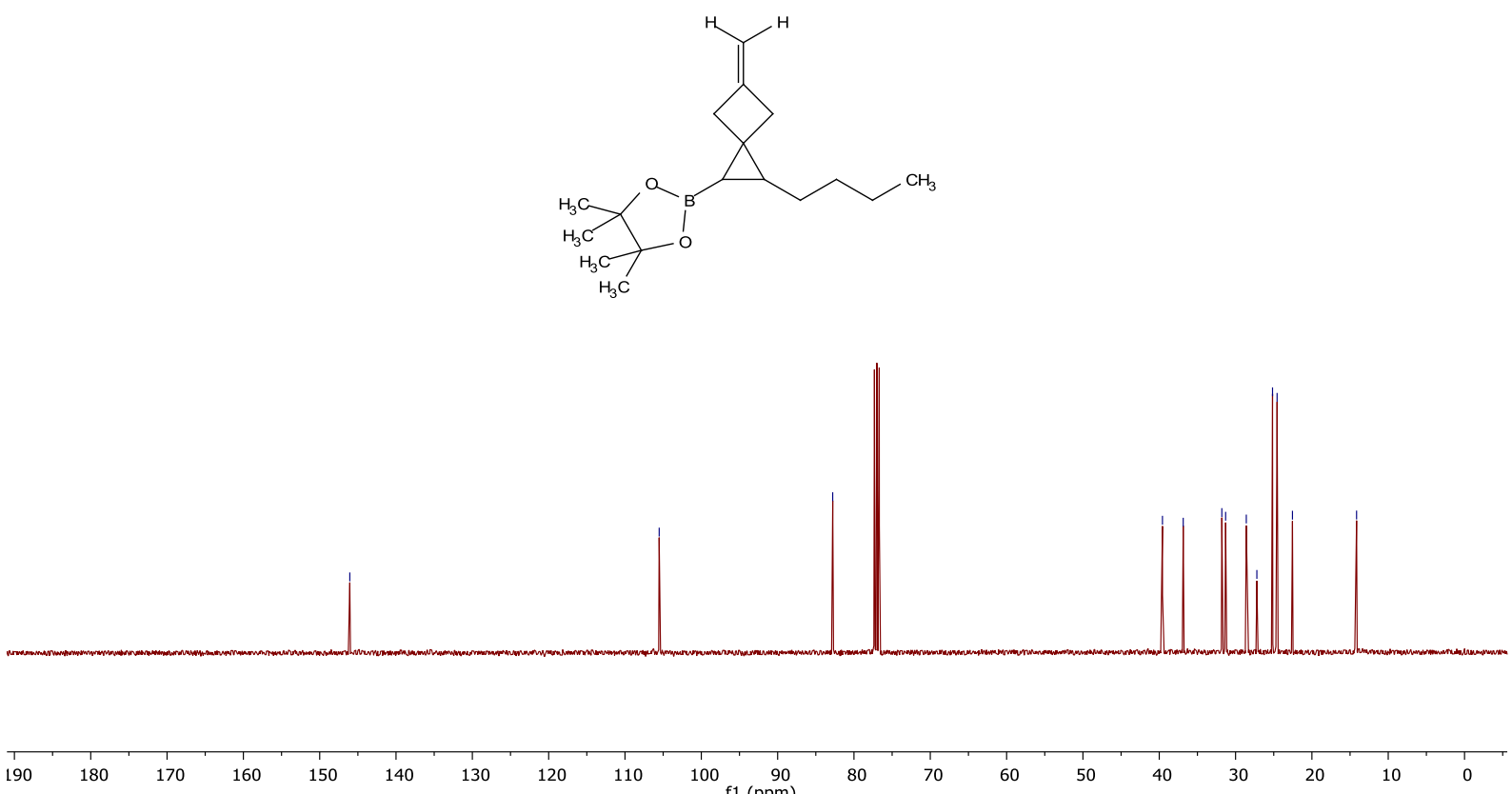
${ }^{11}$ B NMR-12c

44745 yu381.11.fid

$\stackrel{\vec{a}}{\stackrel{m}{n}}$

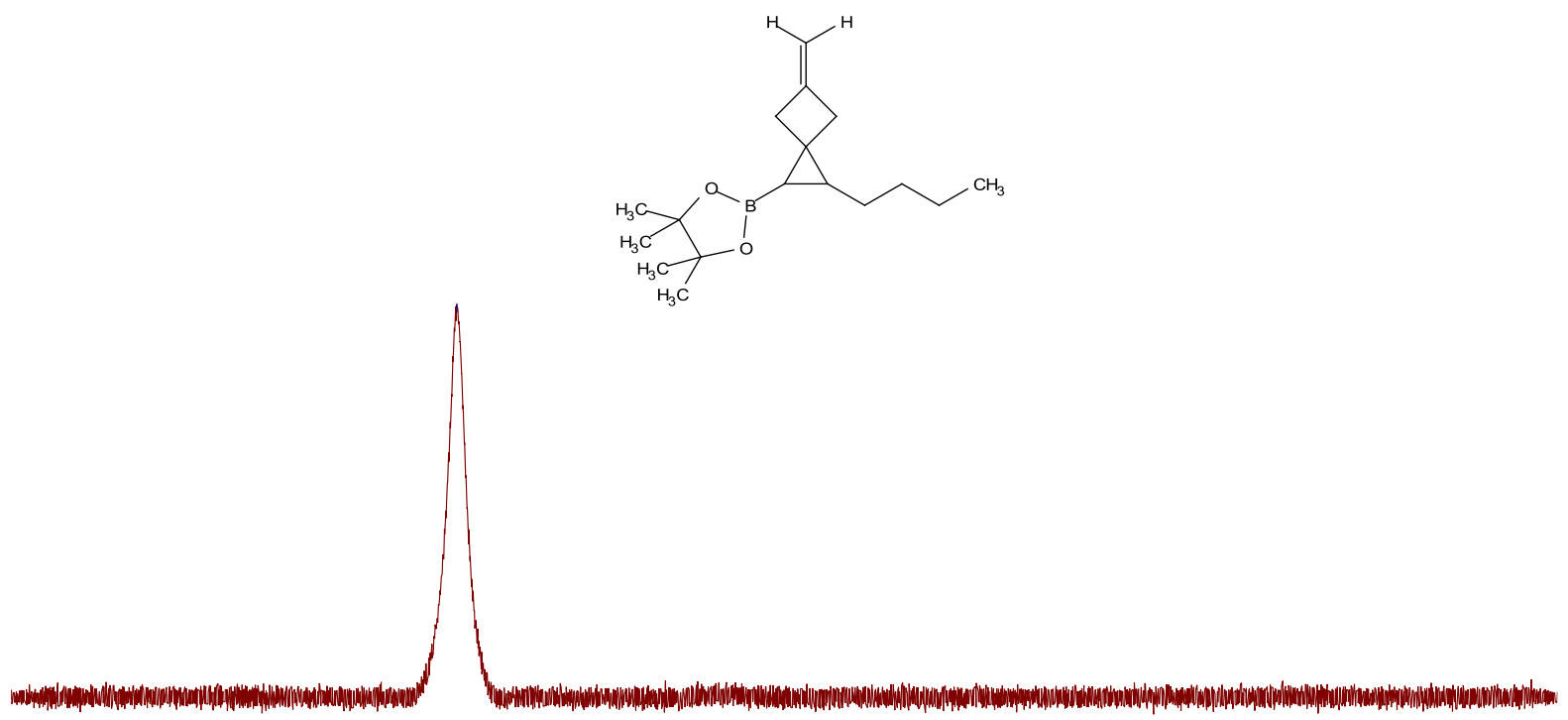

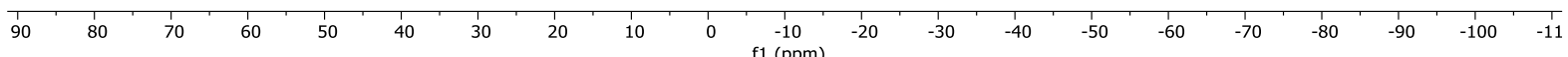



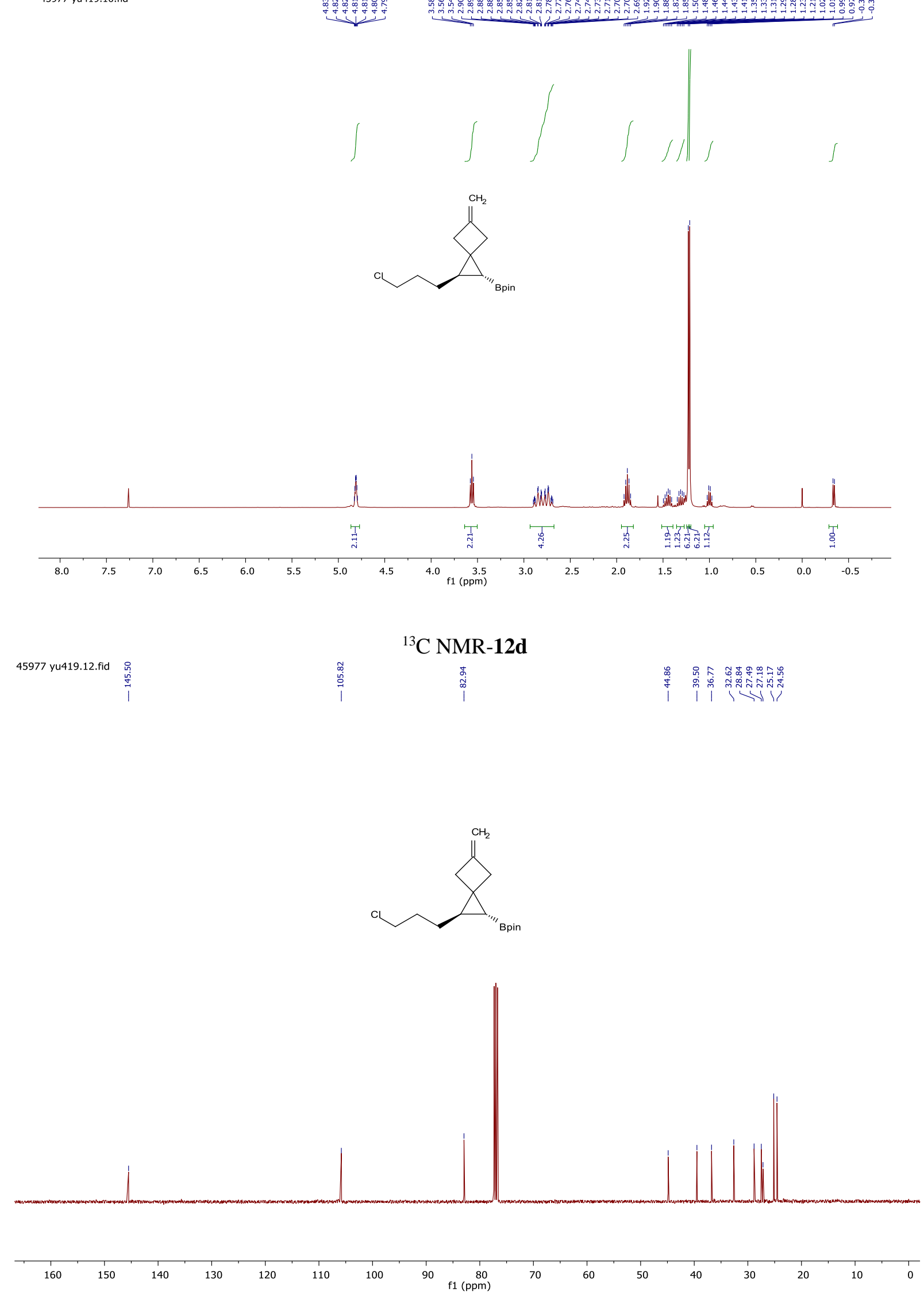

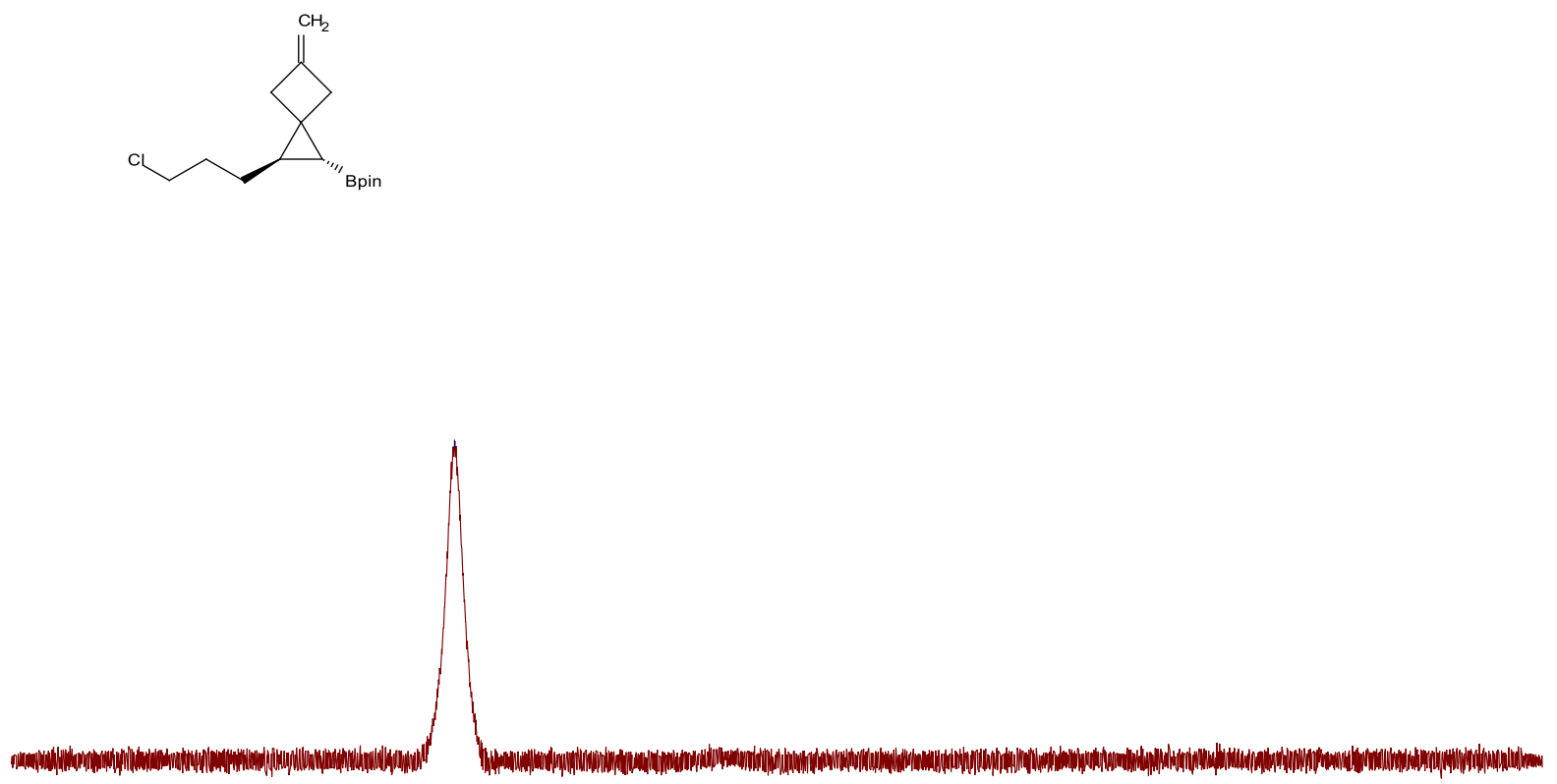

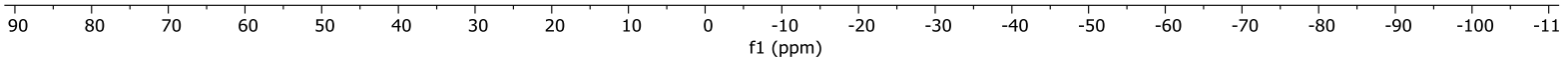




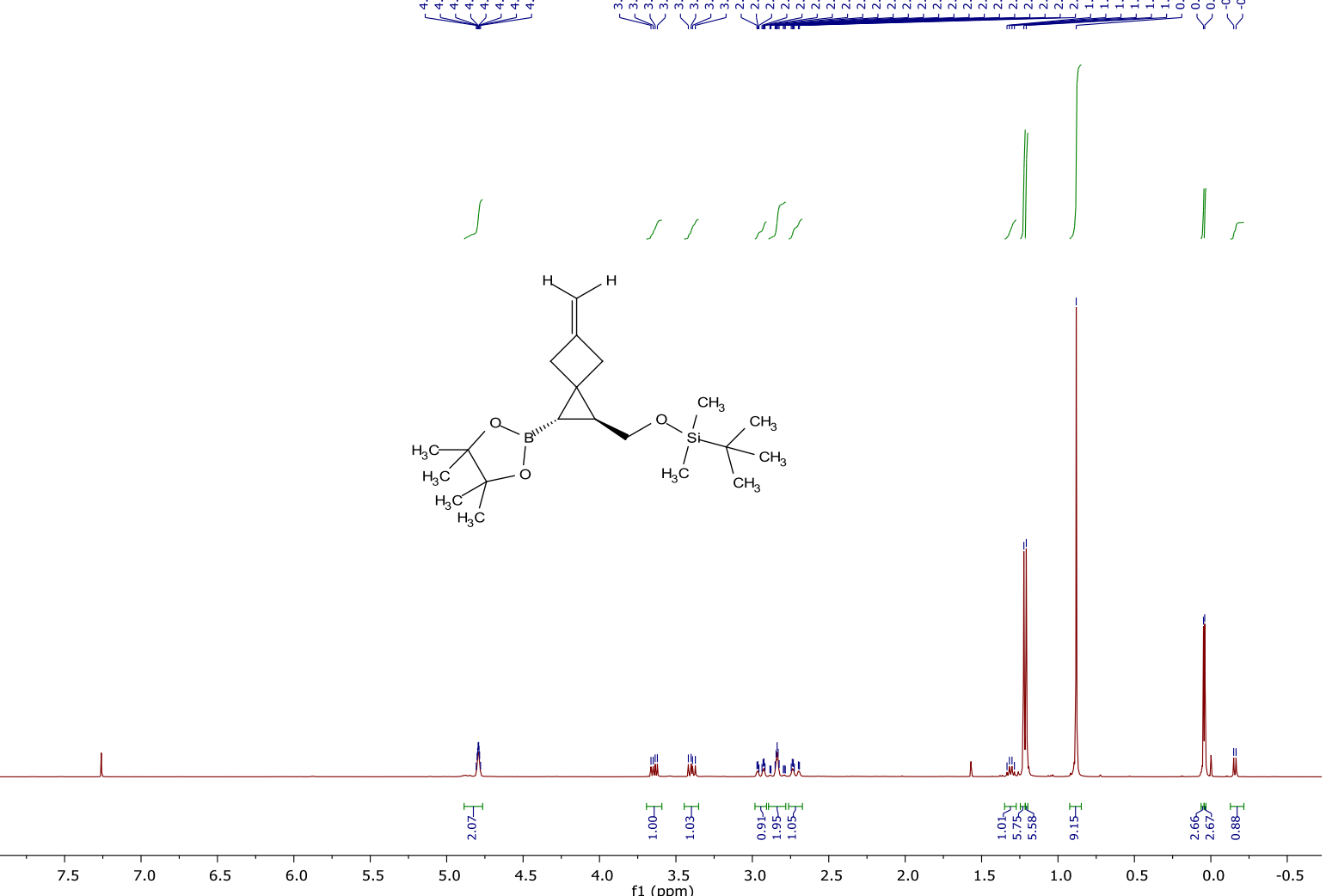

44642 yu378.12.fid
$\substack { \infty \\ \dot{g} \\ \begin{subarray}{c}{j \\ \mid{ \infty \\ \dot { g } \\ \begin{subarray} { c } { j \\ | } } \end{subarray}$

${ }^{13} \mathrm{C}$ NMR-12e

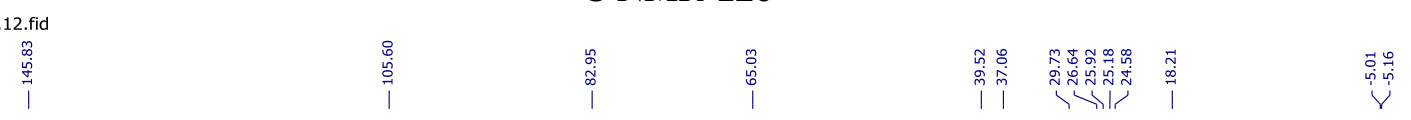

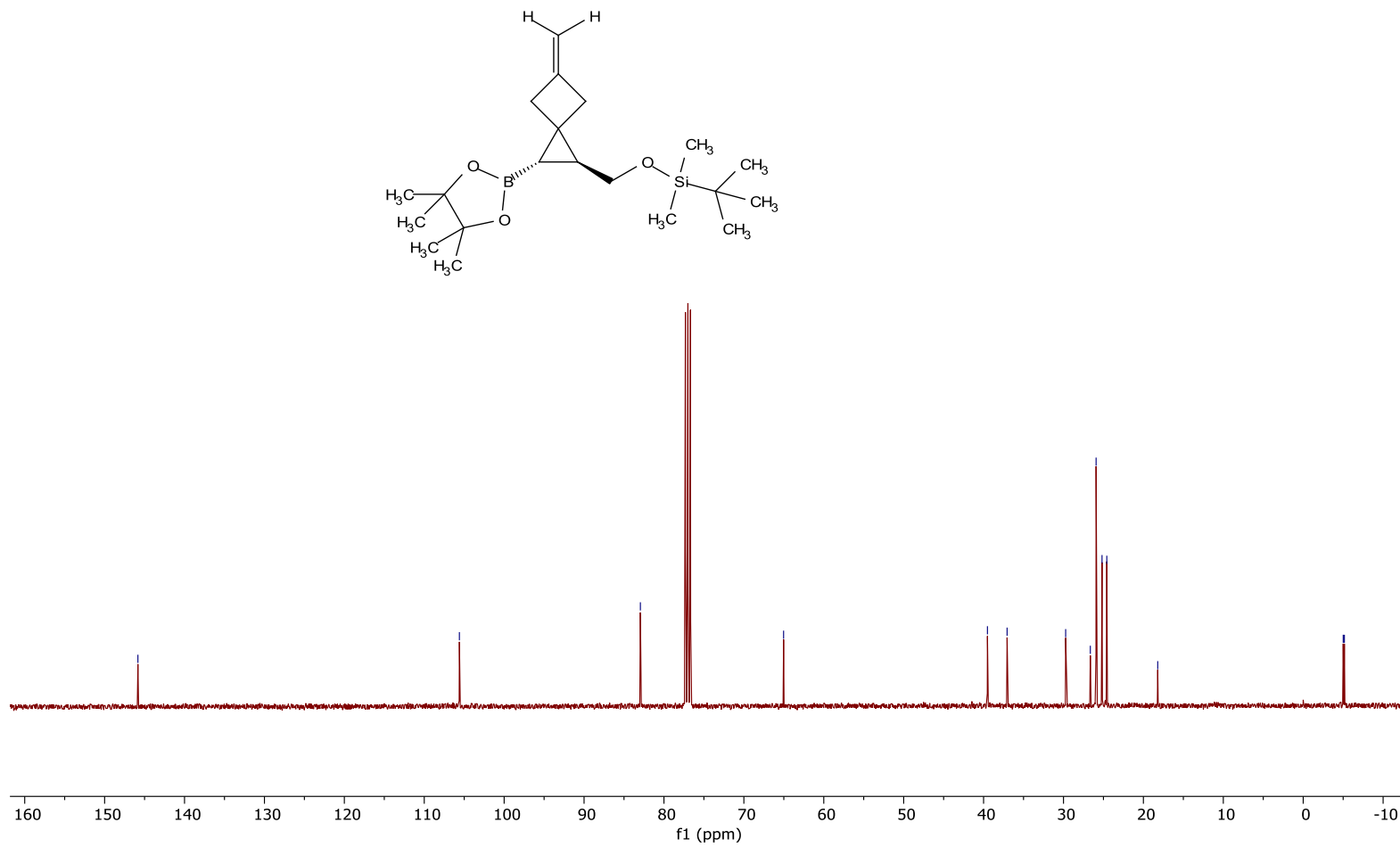



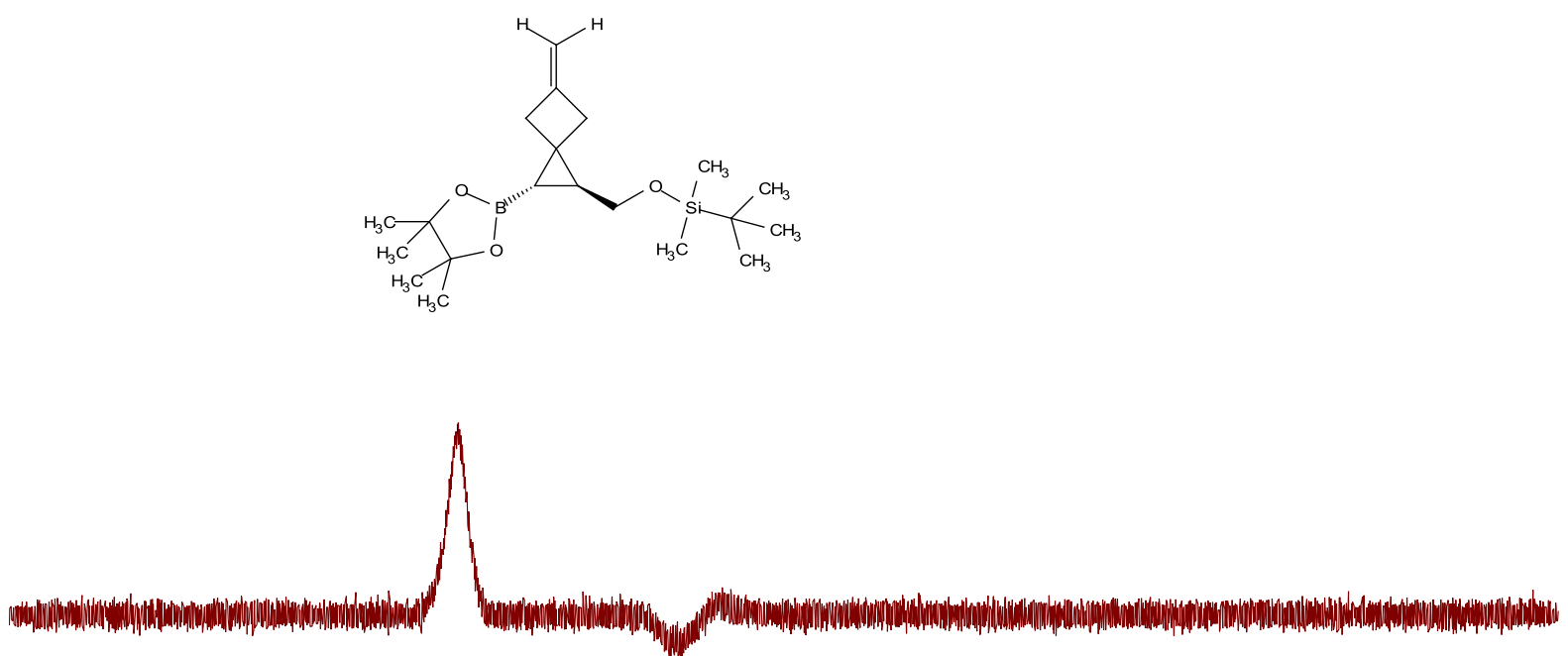

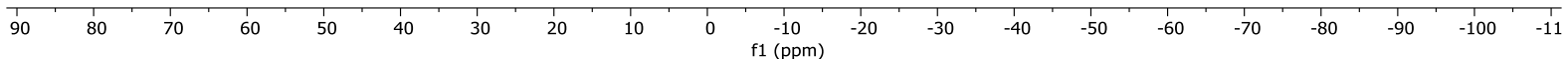




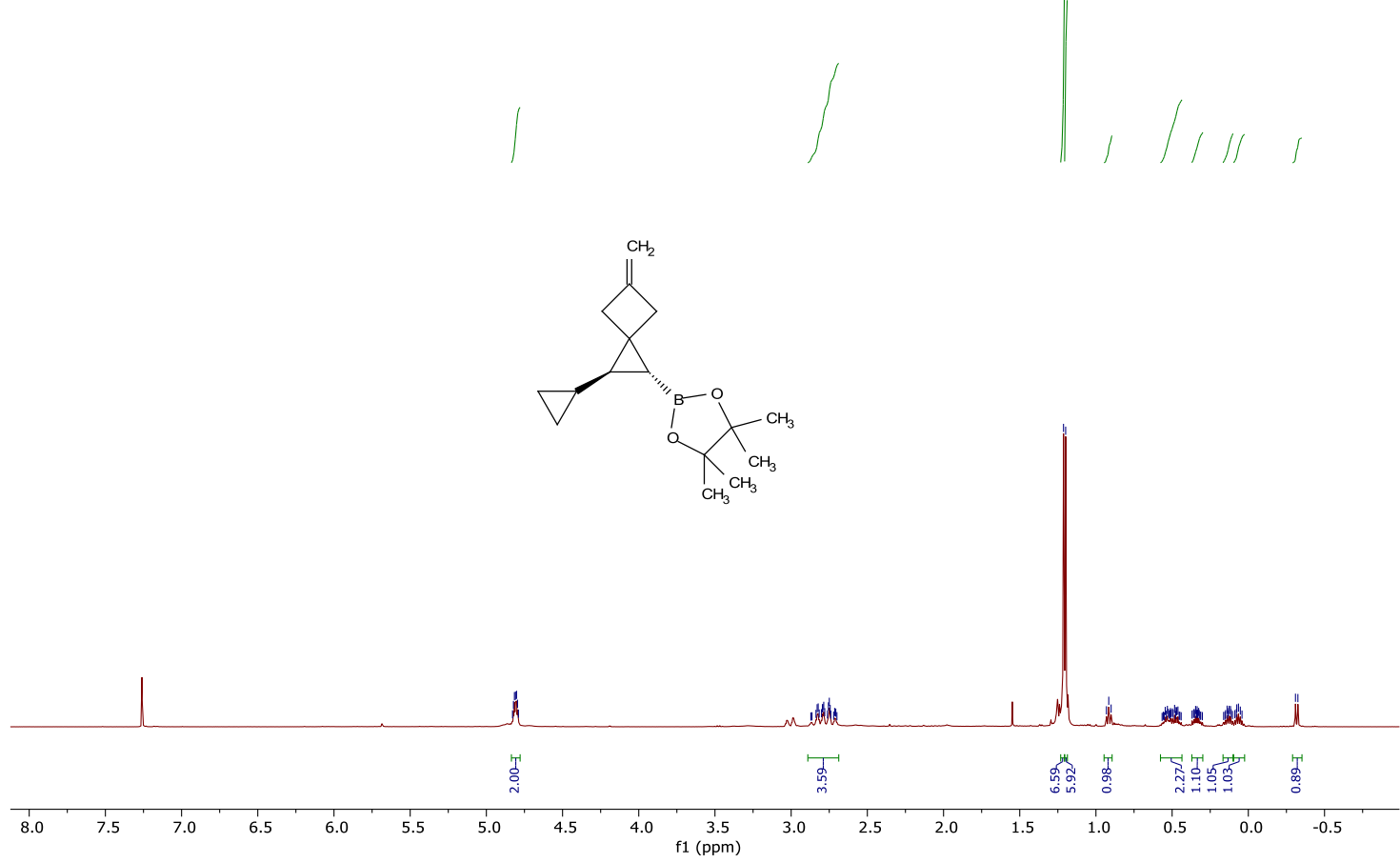

${ }^{13} \mathrm{C}$ NMR-12f

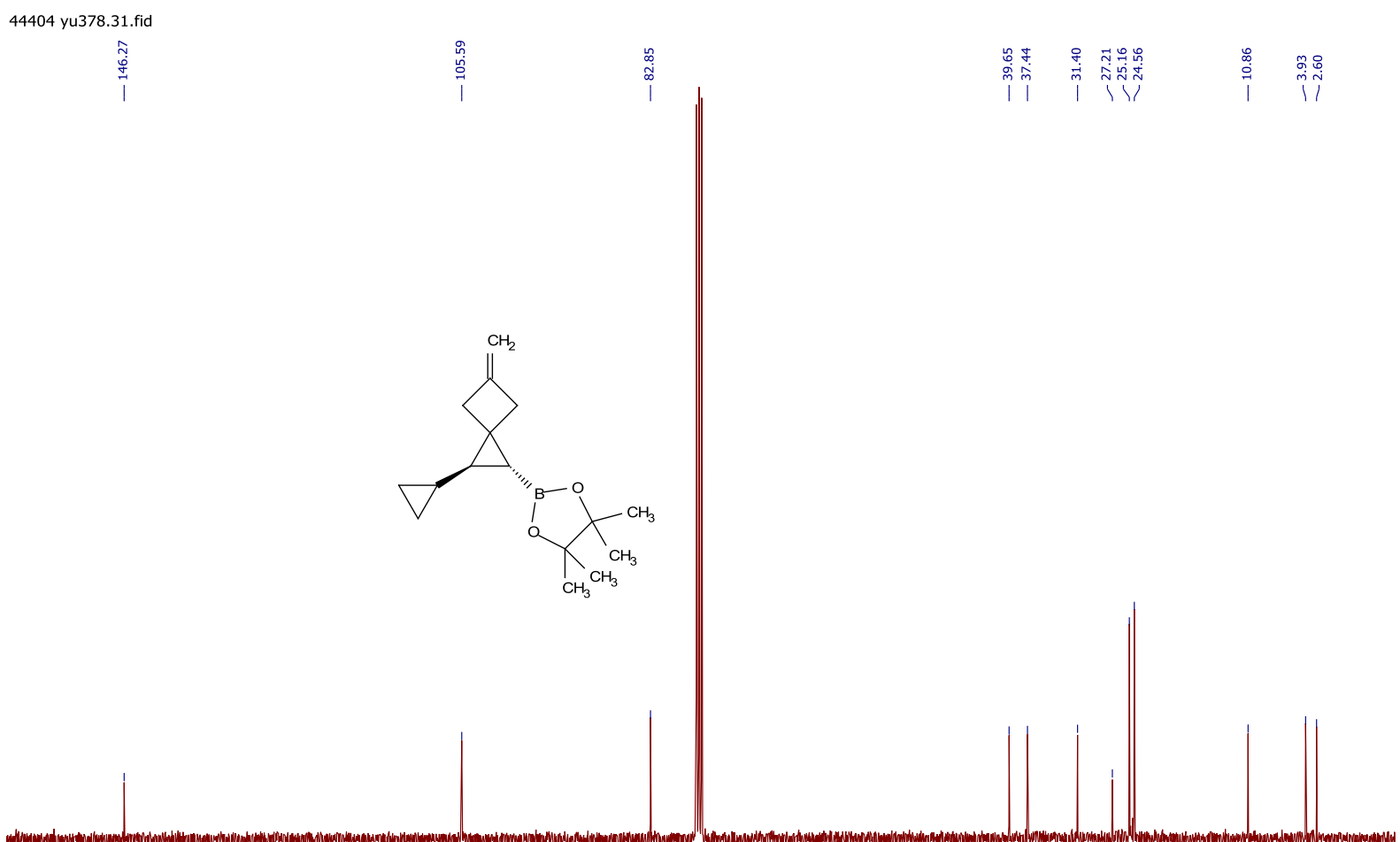

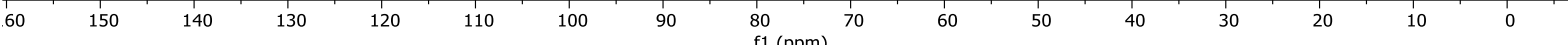


44404 yu378.32.fid

${ }^{11}$ B NMR-12f
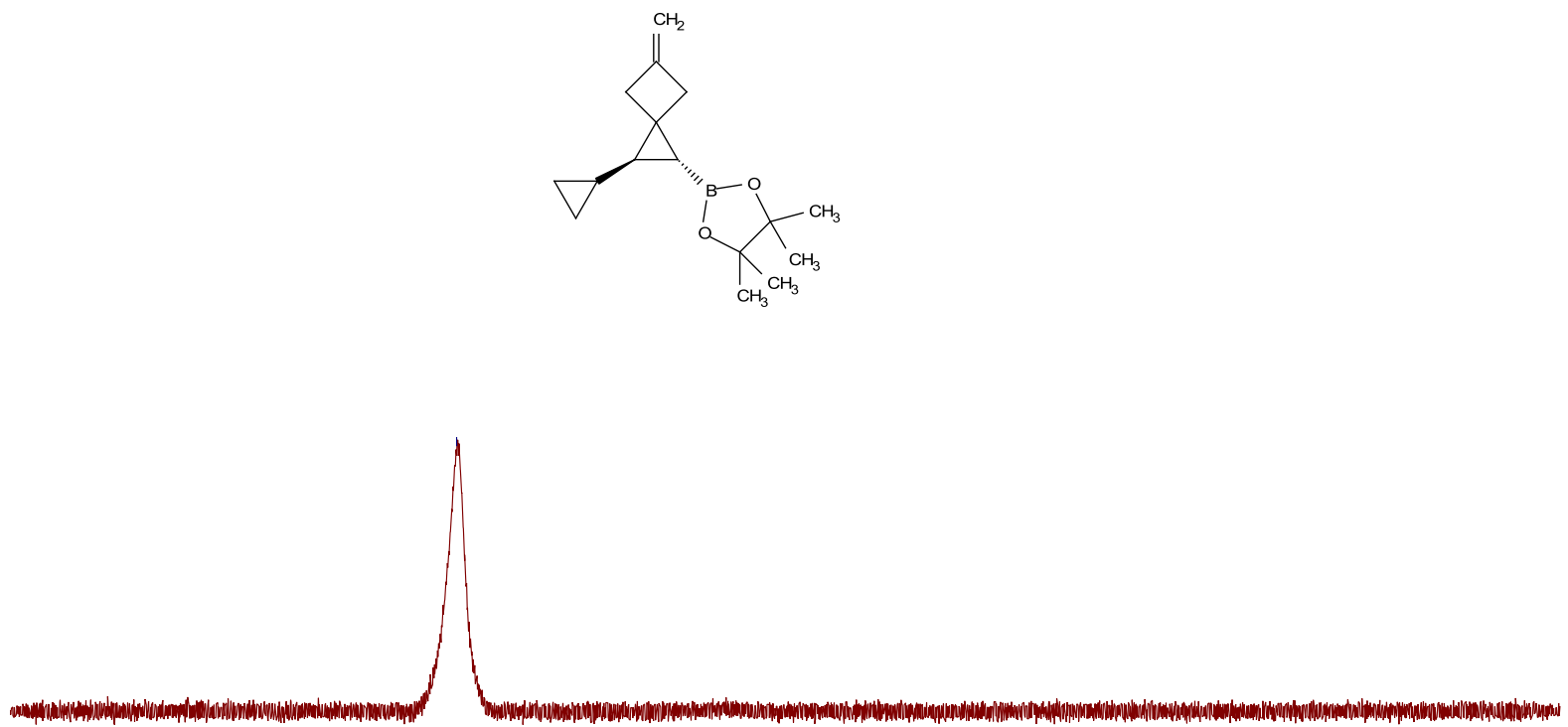

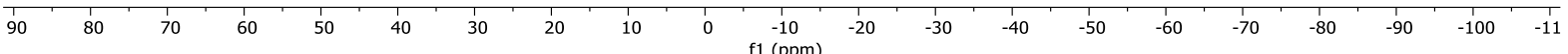


${ }^{1} \mathrm{H}$ NMR-12g

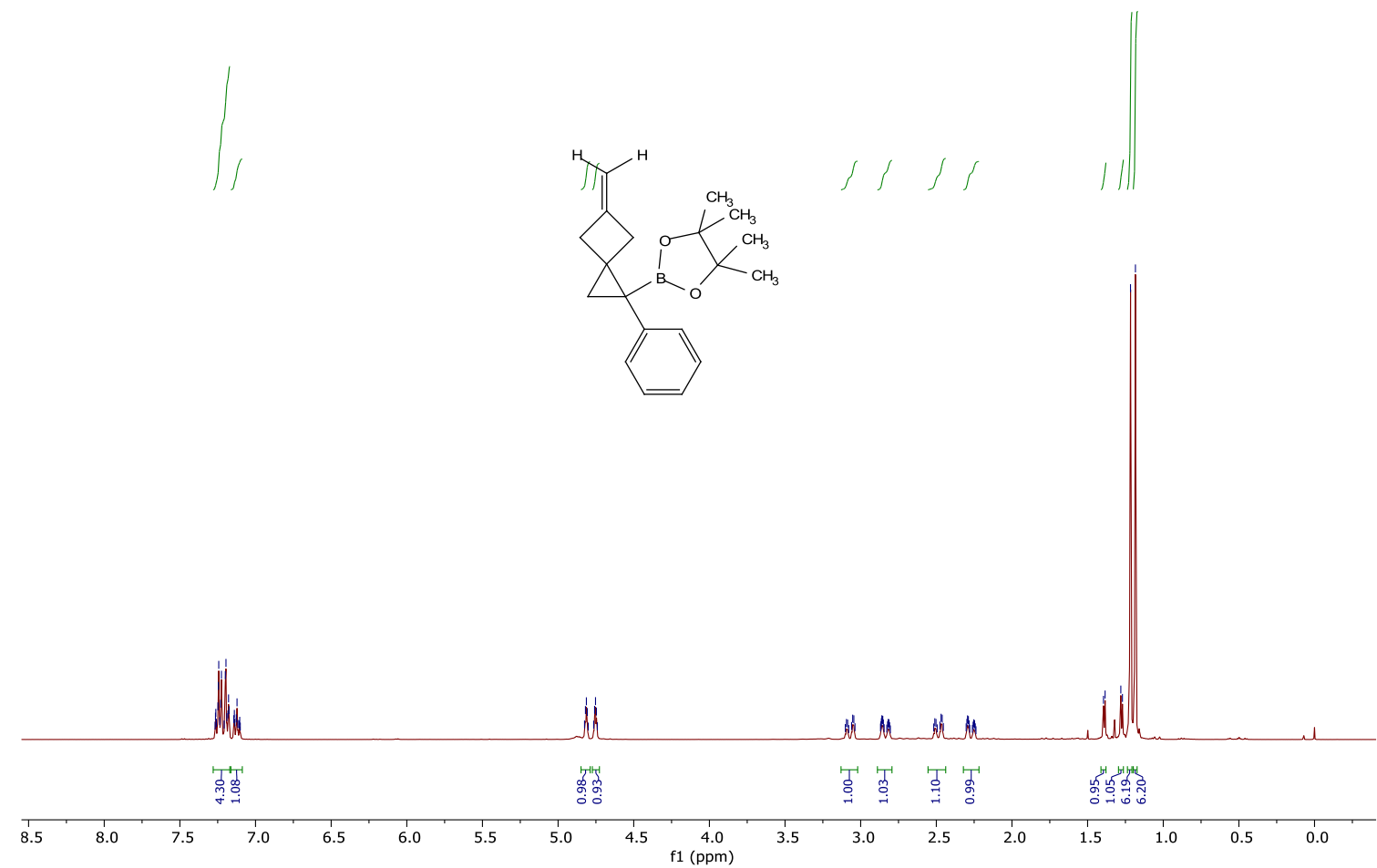

${ }^{13} \mathrm{C}$ NMR-12g

45795 yu415.12 fid

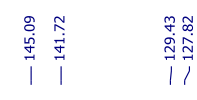

$\stackrel{\circ}{\stackrel{\circ}{\circ}}$

$\underset{\substack{n \\ m}}{\substack{\infty \\ \infty}}$

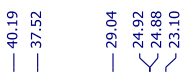
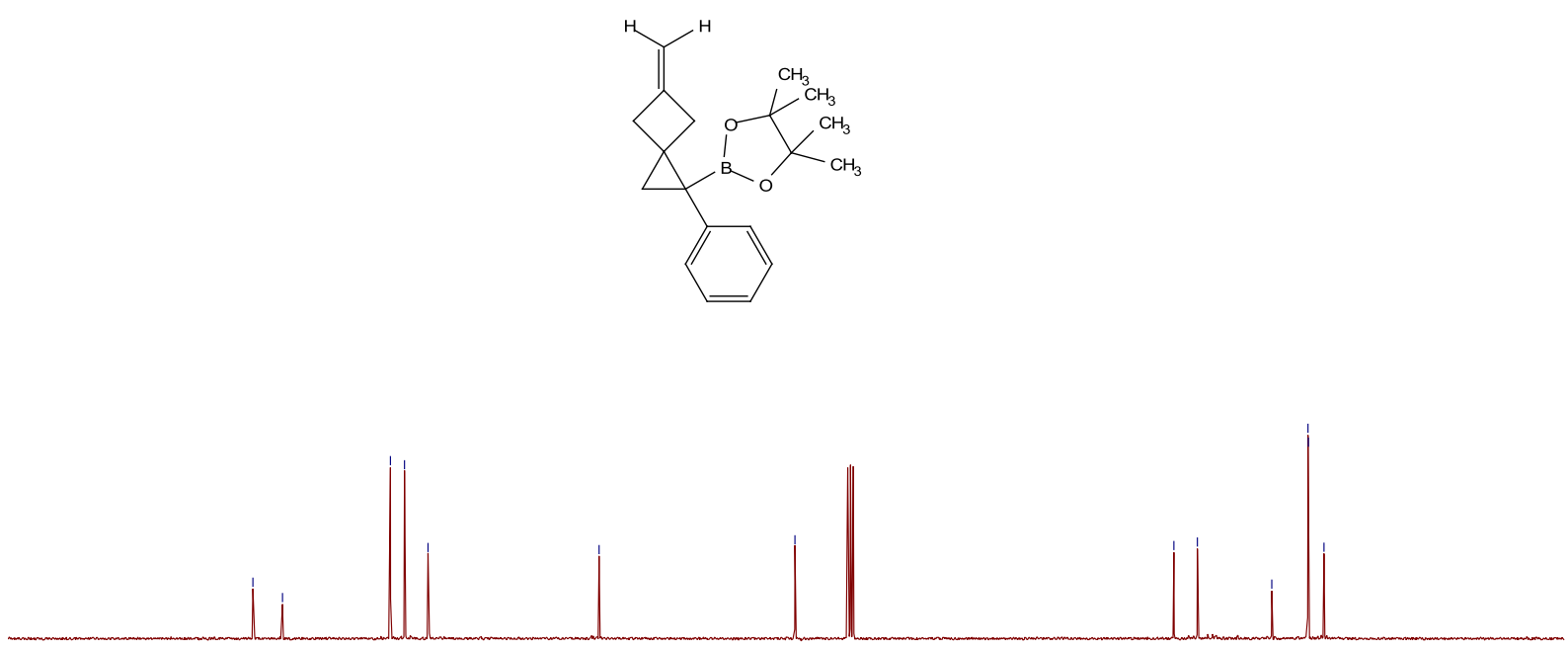

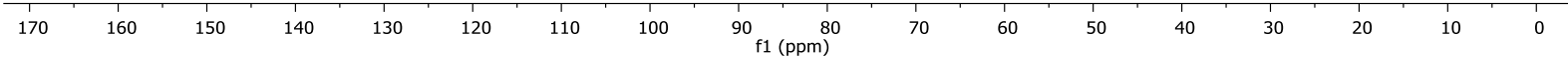


${ }^{11}$ B NMR-12g

45795 yu415.11.fid

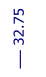
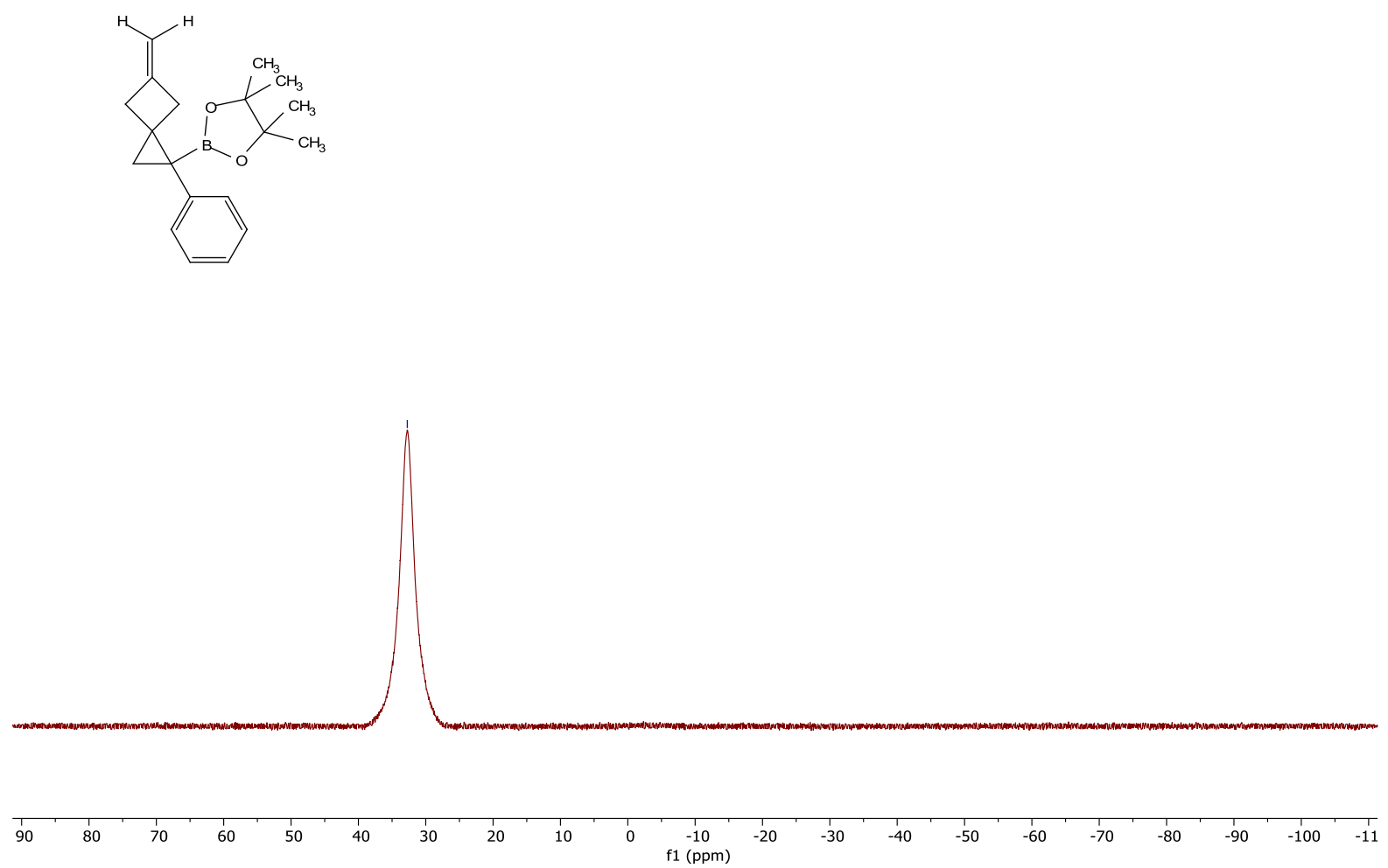
${ }^{1} \mathrm{H}$ NMR-12h

45778 yu412.10.fid

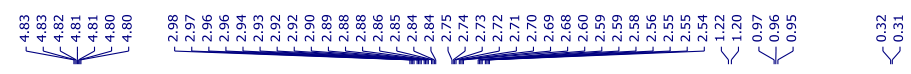

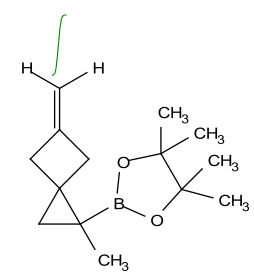

j/IS

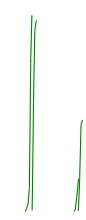

i.

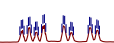

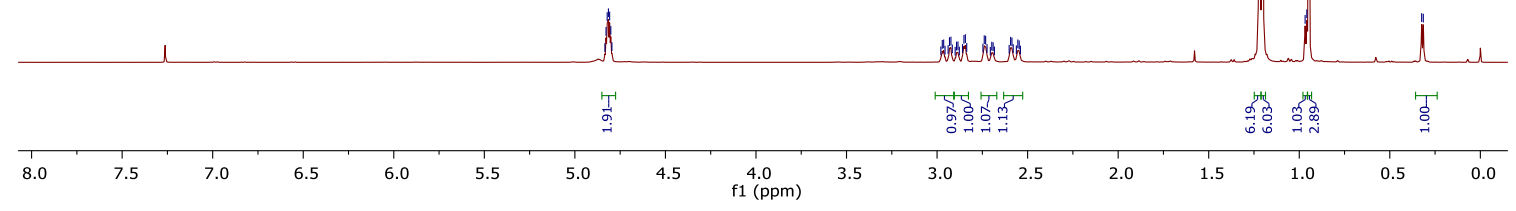

${ }^{13}$ C NMR-12h

45778 yu412.12.fid $\stackrel{\substack { m \\ \begin{subarray}{c}{j \\ j{ m \\ \begin{subarray} { c } { j \\ j } }\end{subarray}}{1}$
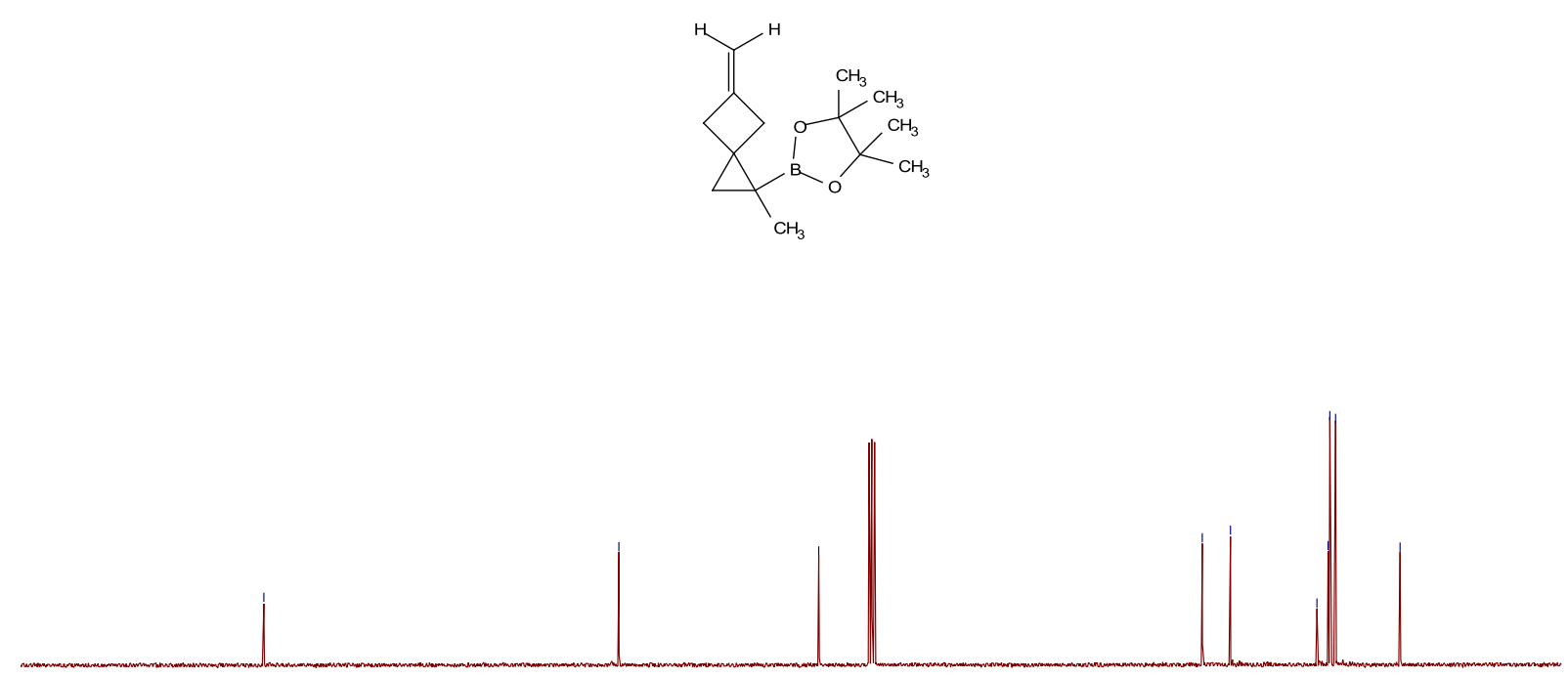

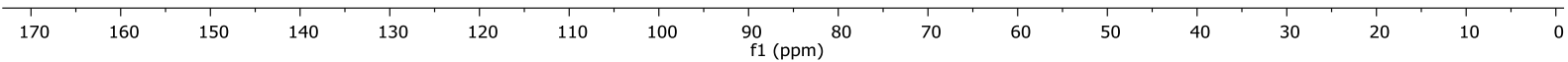


${ }^{11}$ B NMR-12h

45778 yu412.11.fid

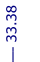<smiles>CC1(B2CC3(CC3)C(C)(C)O2)CC1</smiles>

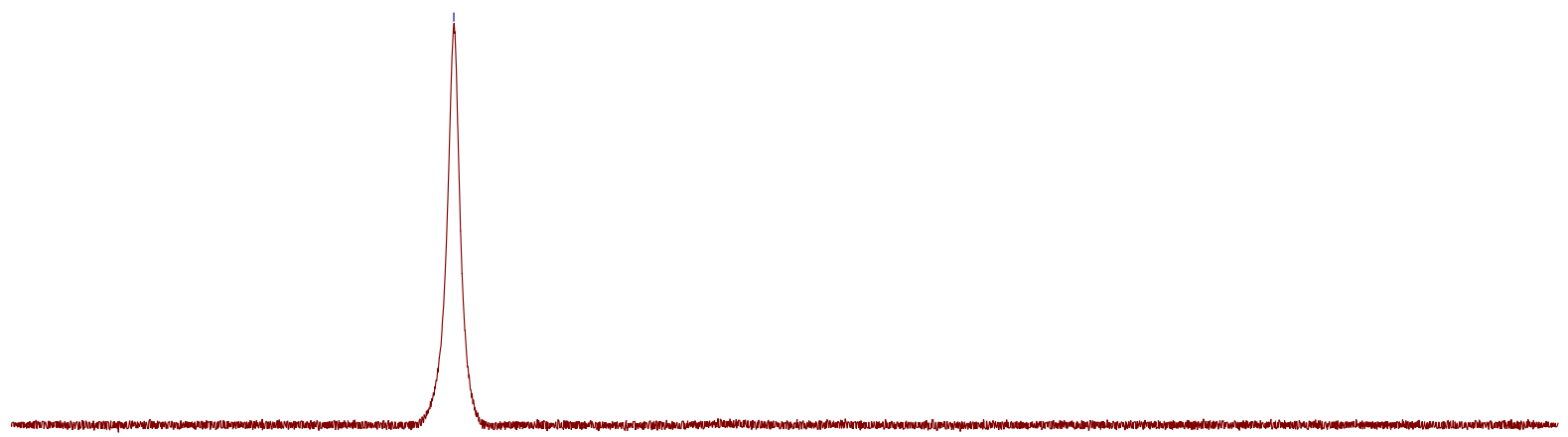

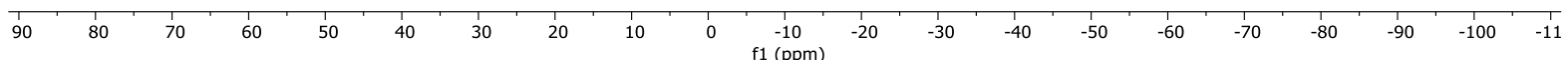


${ }^{1} \mathrm{H}$ NMR-12i

45981 yu420.10.fid

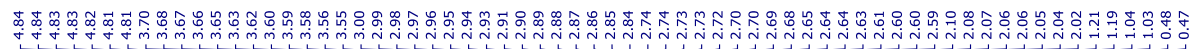
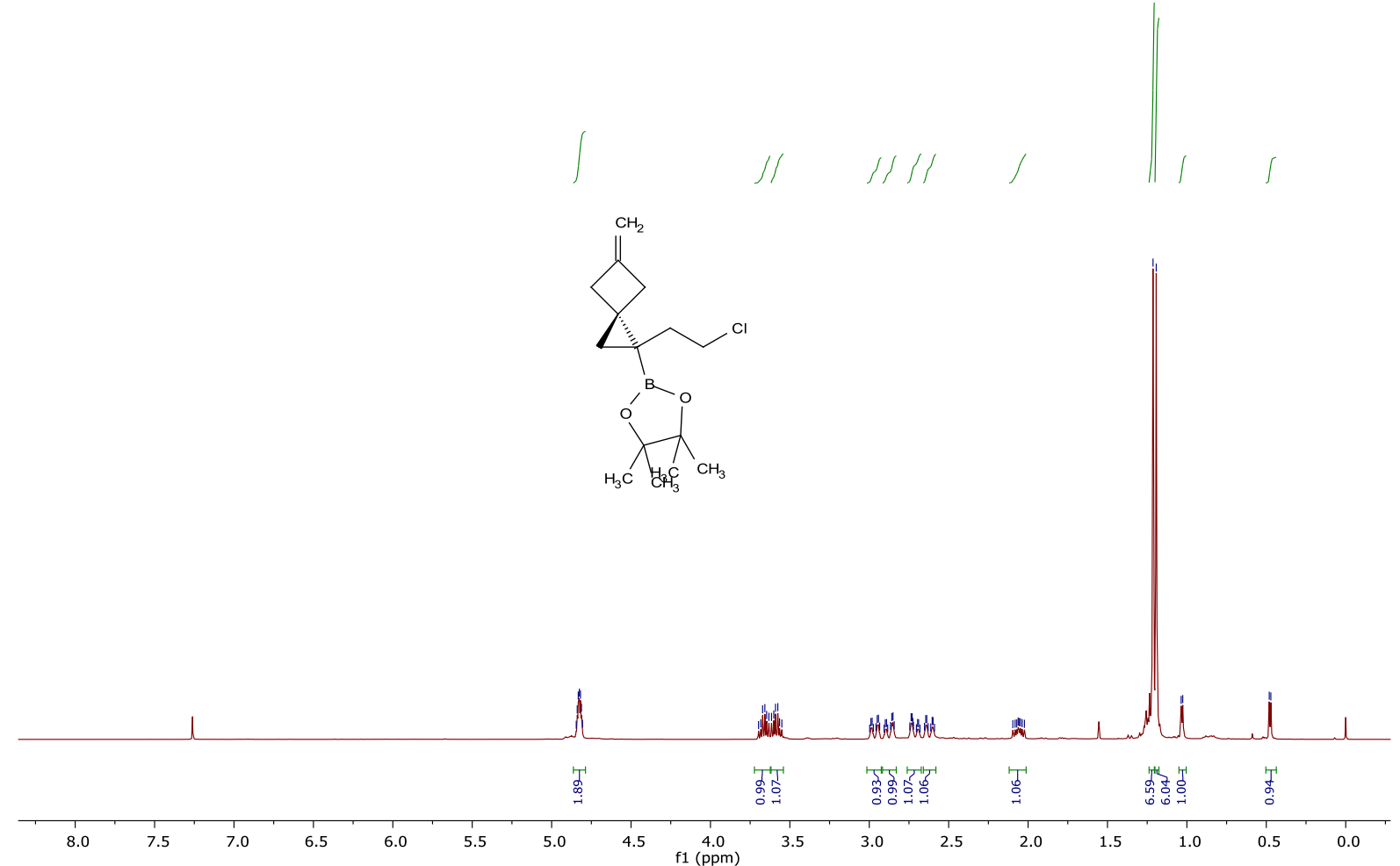

${ }^{13} \mathrm{C}$ NMR-12i

45981 yu420.12.fid

$\infty$
$\infty$
$j$
$j$
$j$

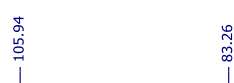

IVI

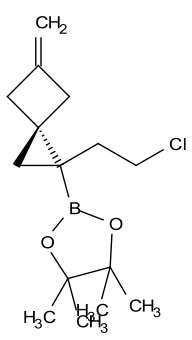

$\underset{\substack{\infty \\ \infty}}{\substack{\infty \\ \text { I }}}$

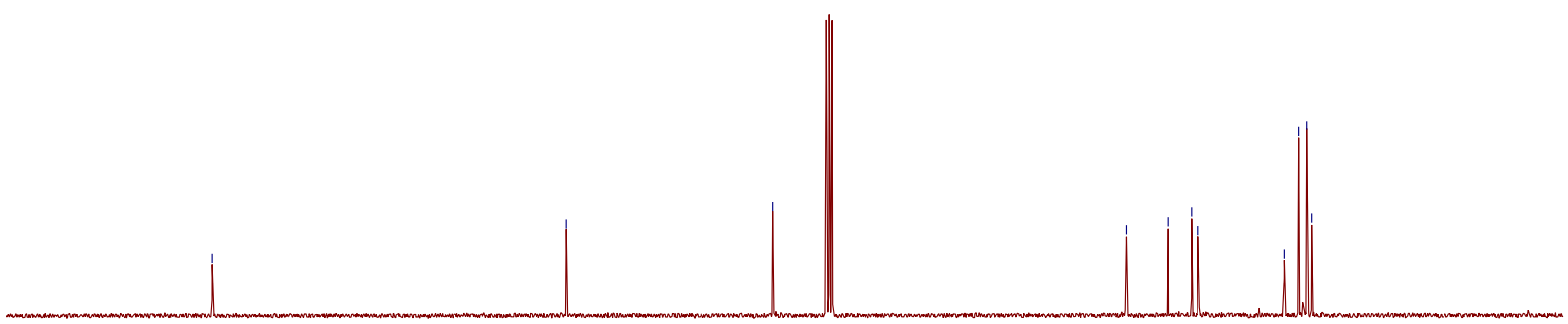

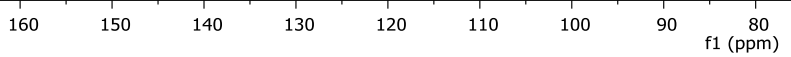


${ }^{11}$ B NMR-12i

45981 yu420.11.fid

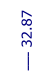
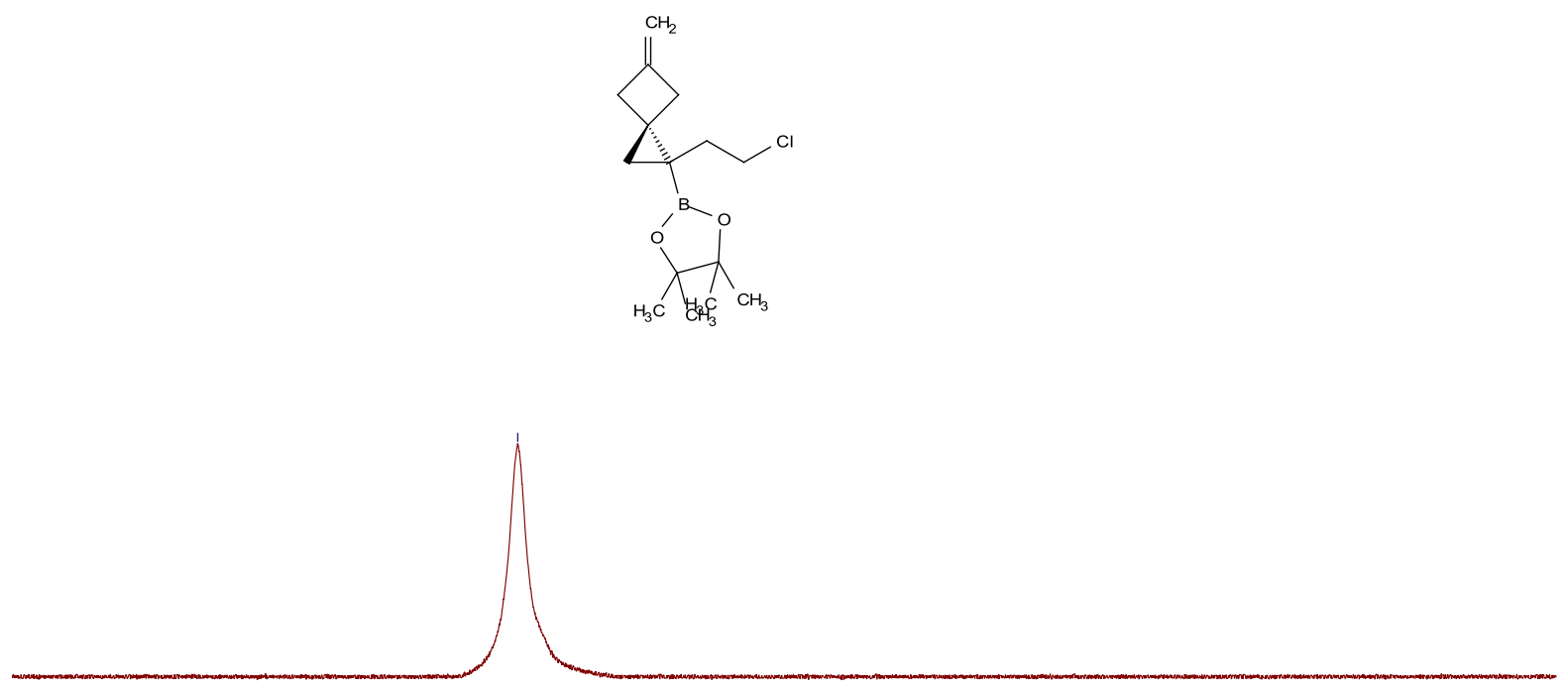

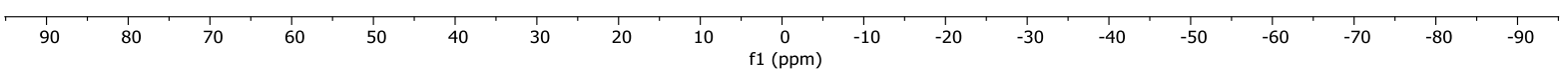


45251 yu390.10.fid

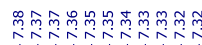

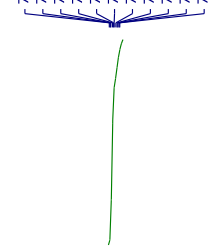

${ }^{1} \mathrm{H}$ NMR-13

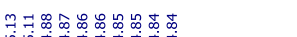
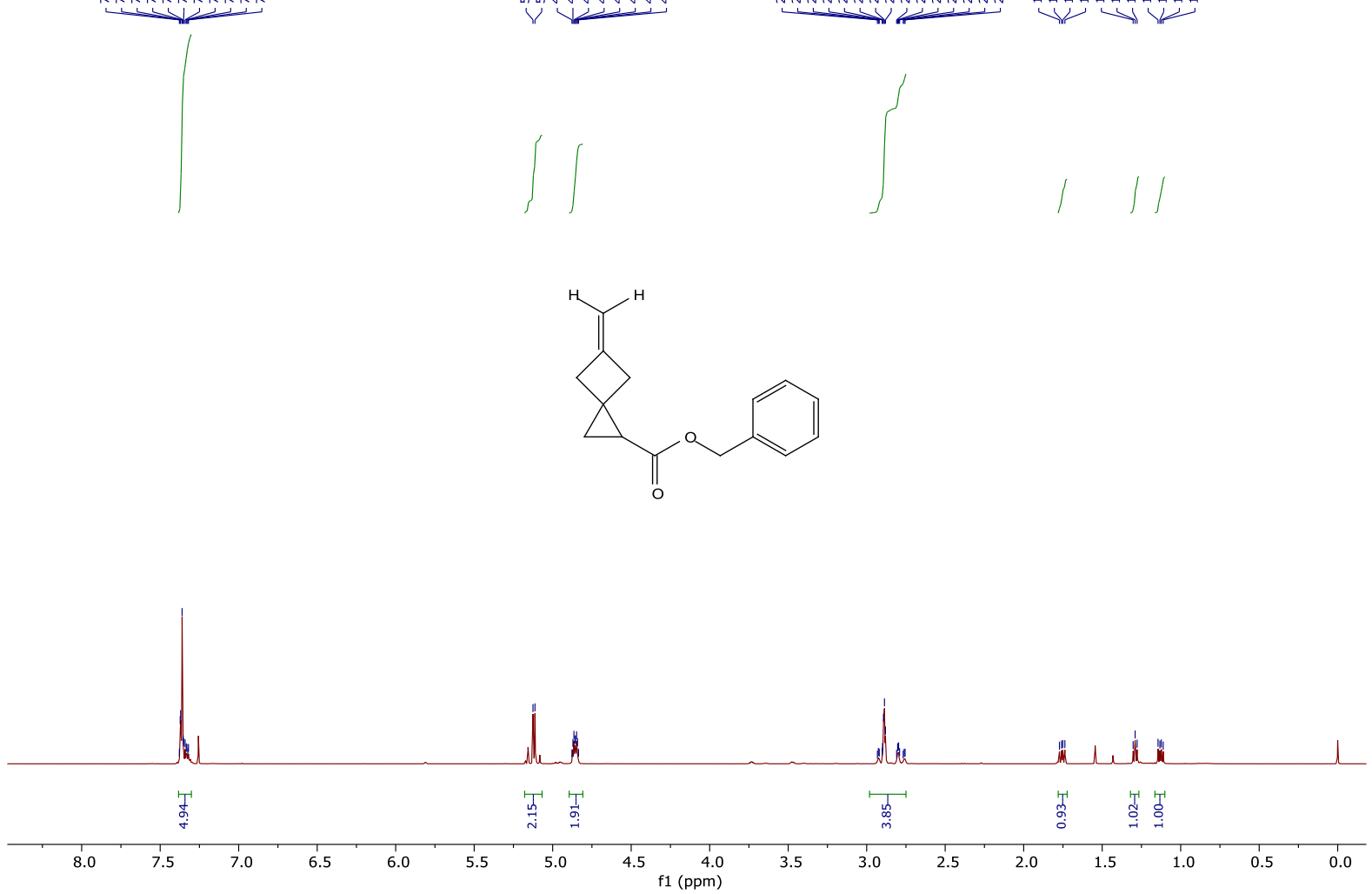

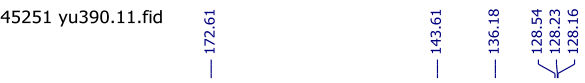

${ }^{13} \mathrm{C}$ NMR-13
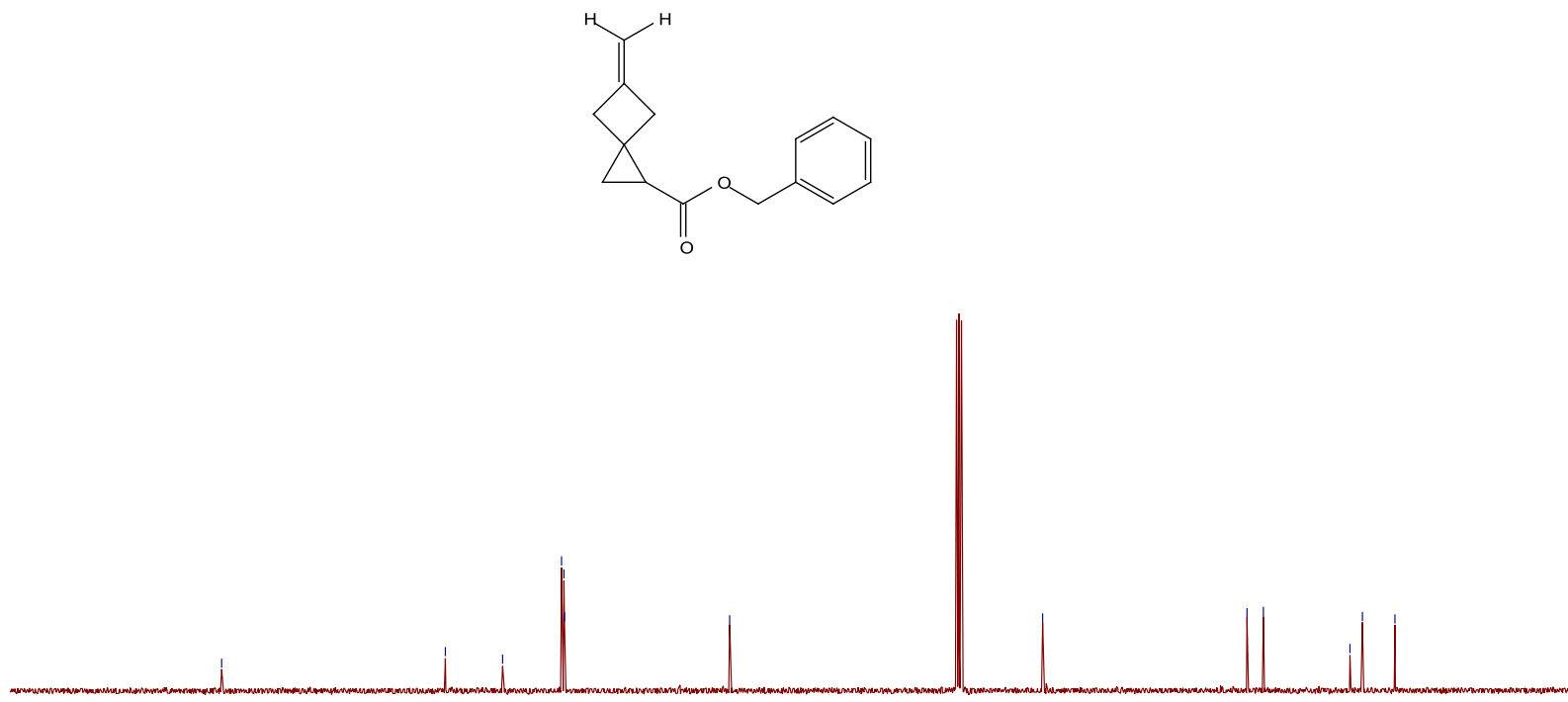

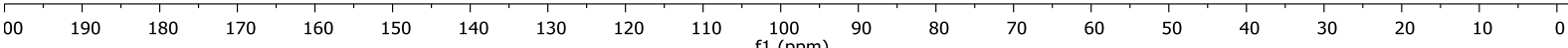



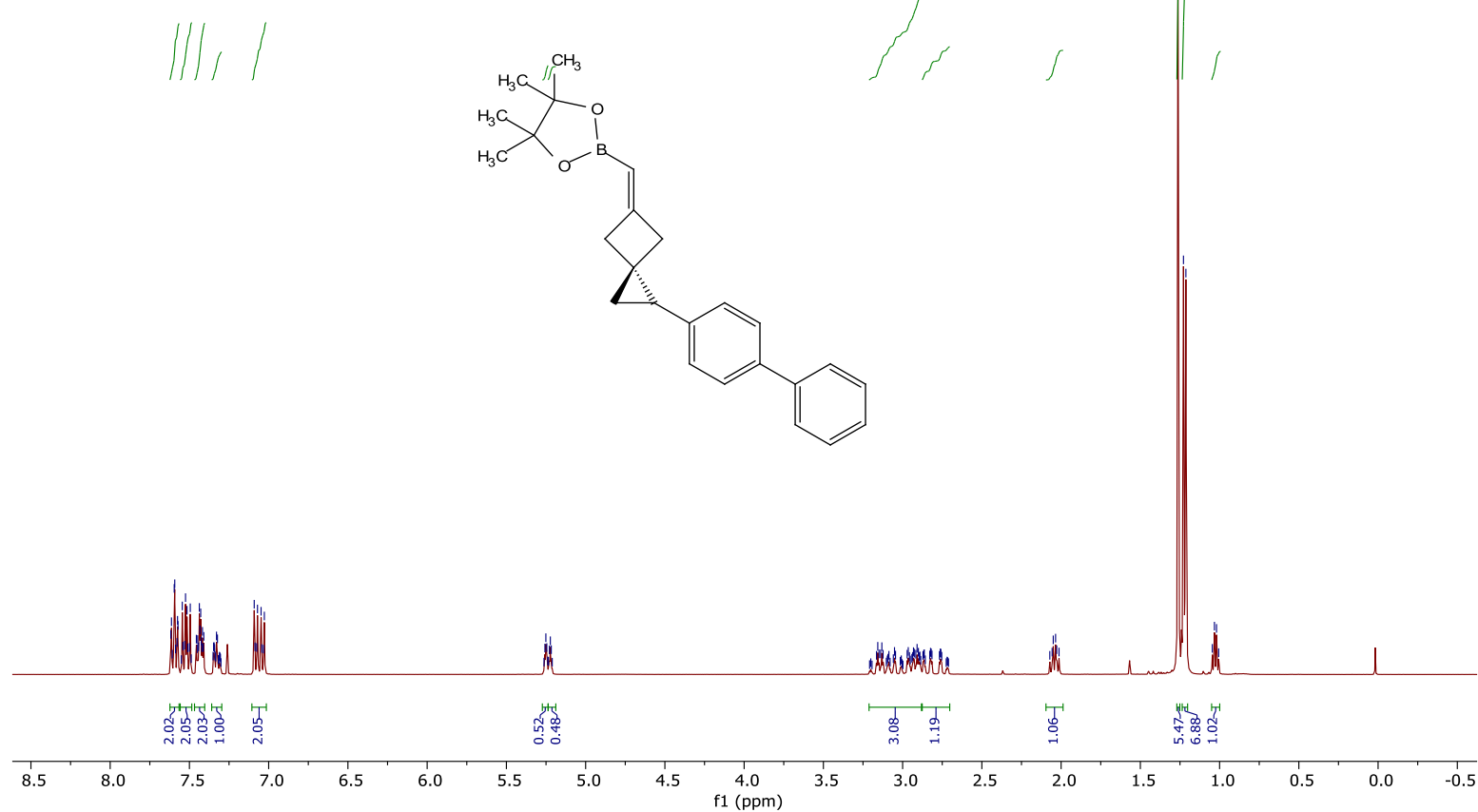

${ }^{13} \mathrm{C}$ NMR-14

45630 yu409.12.fid

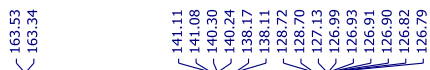

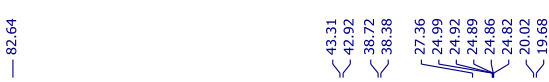

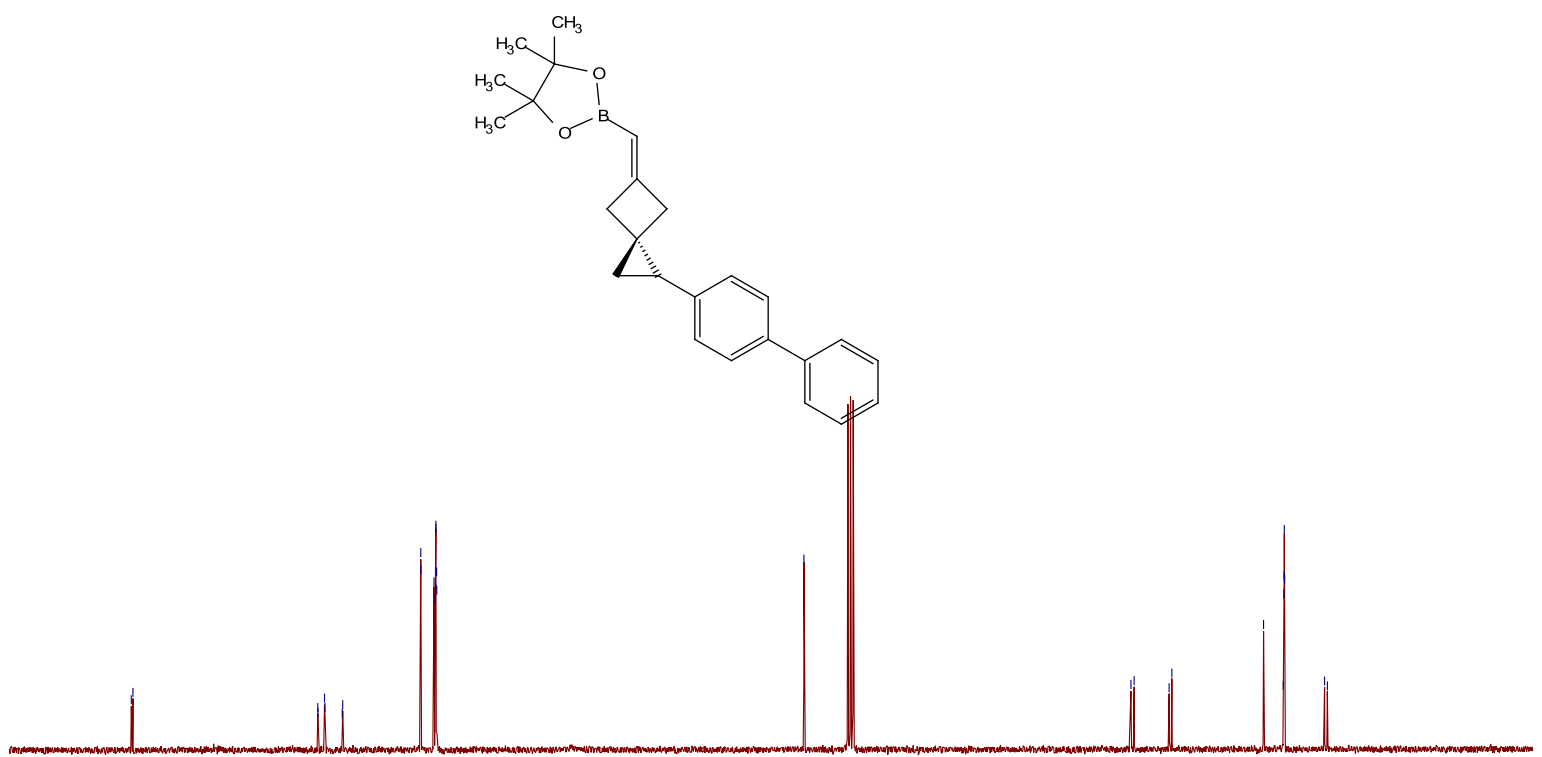

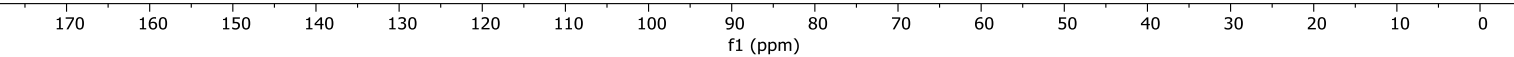


${ }^{11}$ B NMR-14

45630 yu 409.11. fid

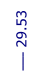

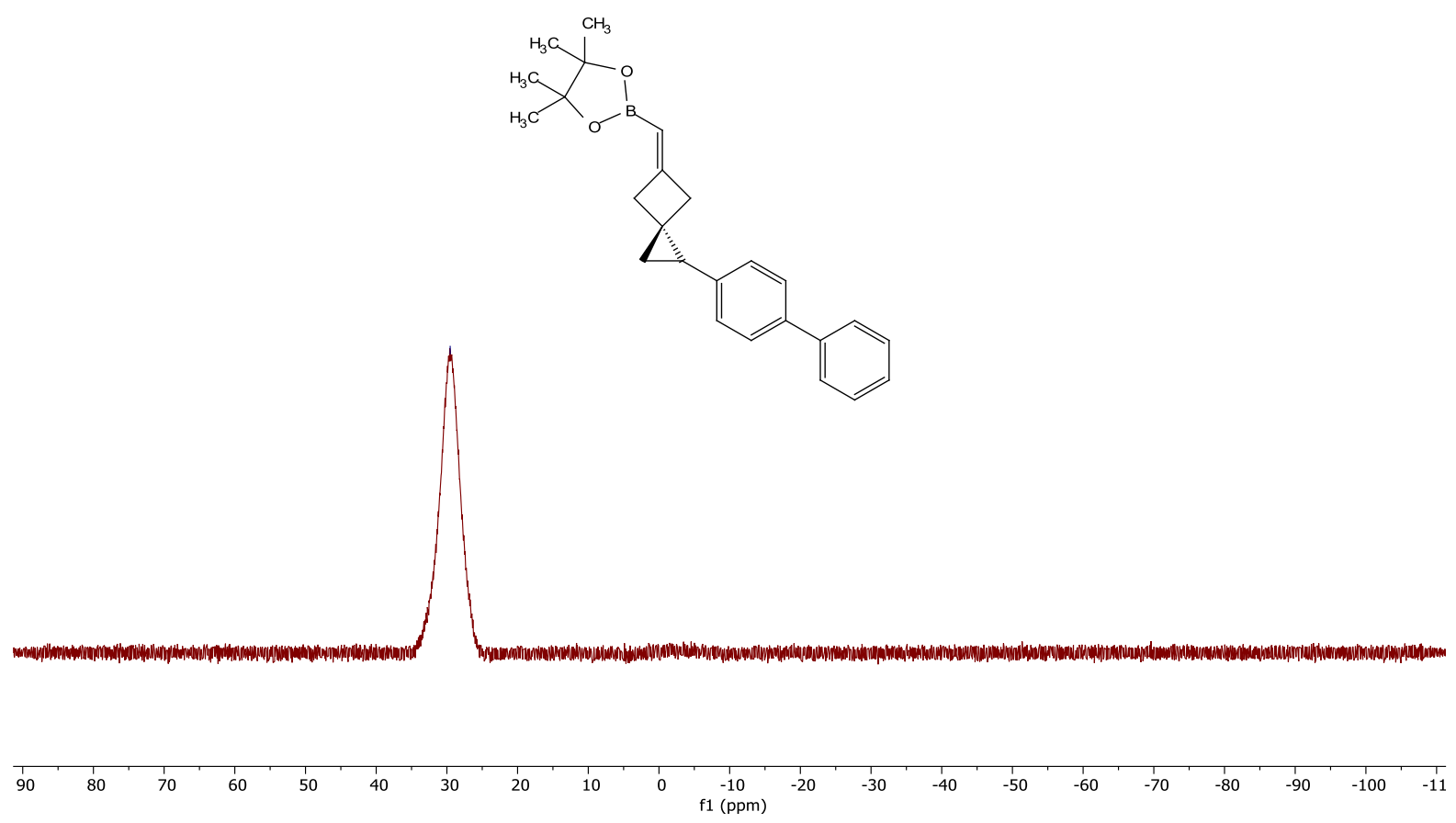

S111 

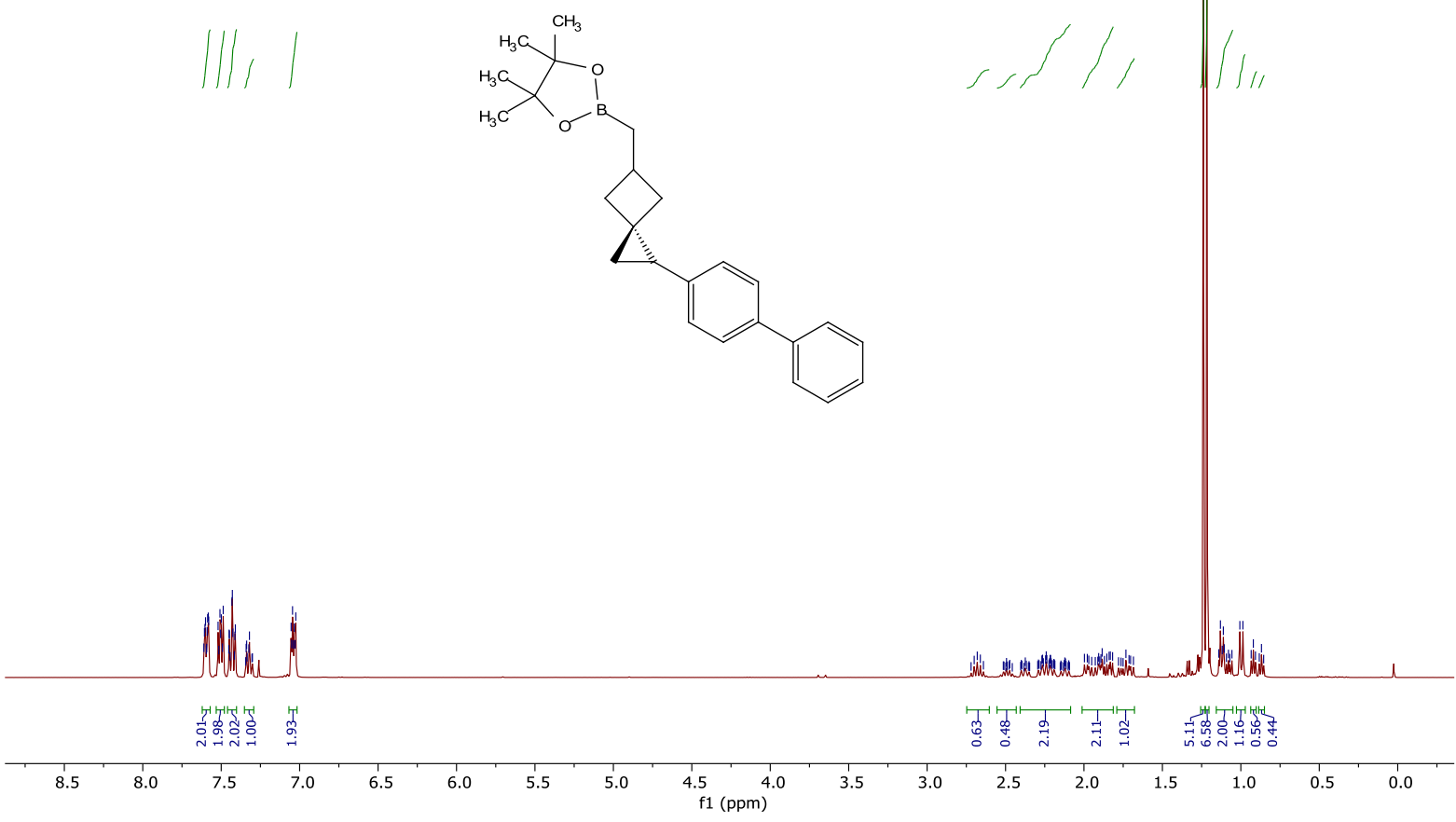

${ }^{13} \mathrm{C}$ NMR-15 45624 yu406.11.fid

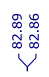

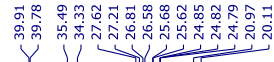

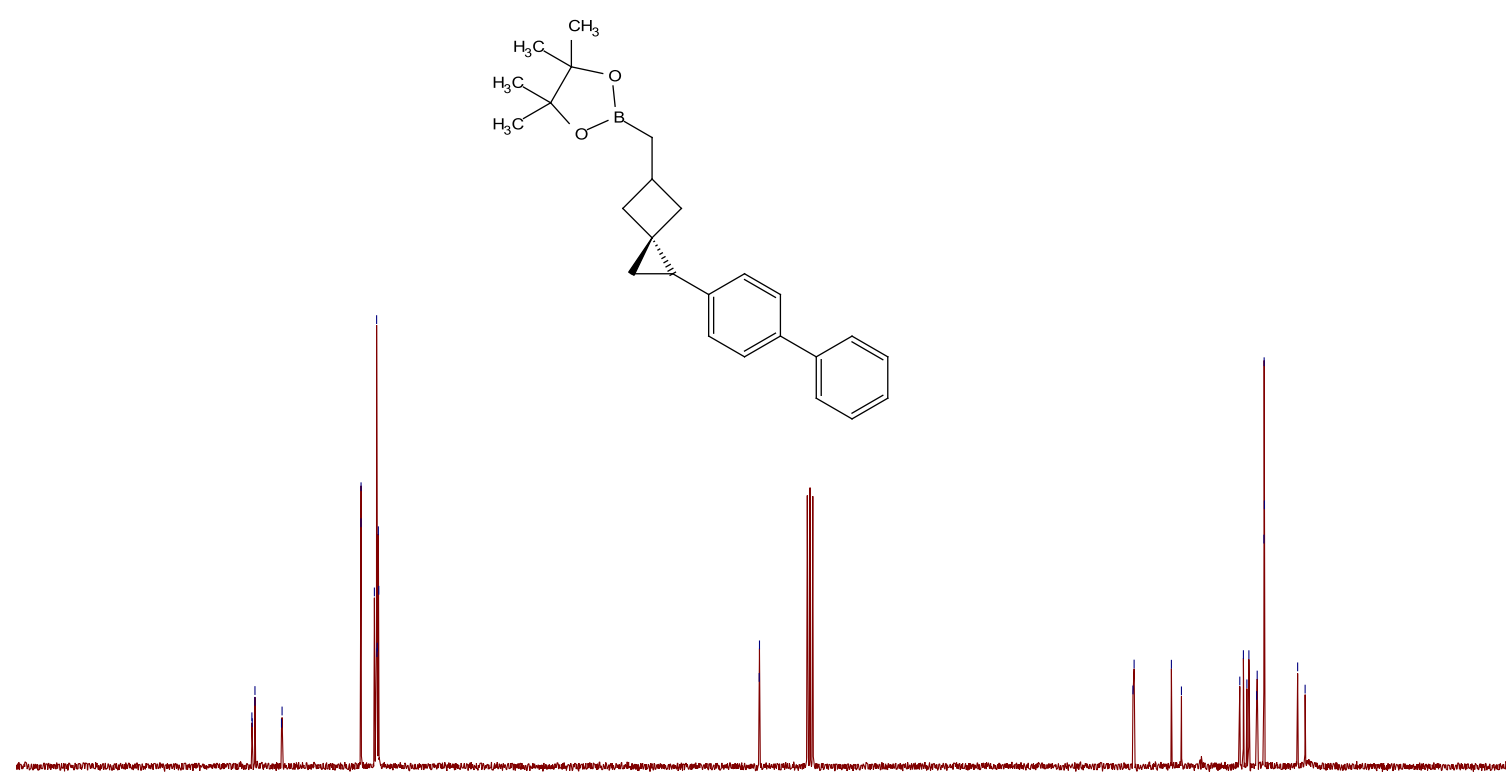

$\begin{array}{lllllllll}160 & 150 & 140 & 130 & 120 & 110 & 100 & 90 & 80\end{array}$ 
${ }^{11}$ B NMR-15

45620 yu406.11.fid

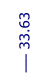

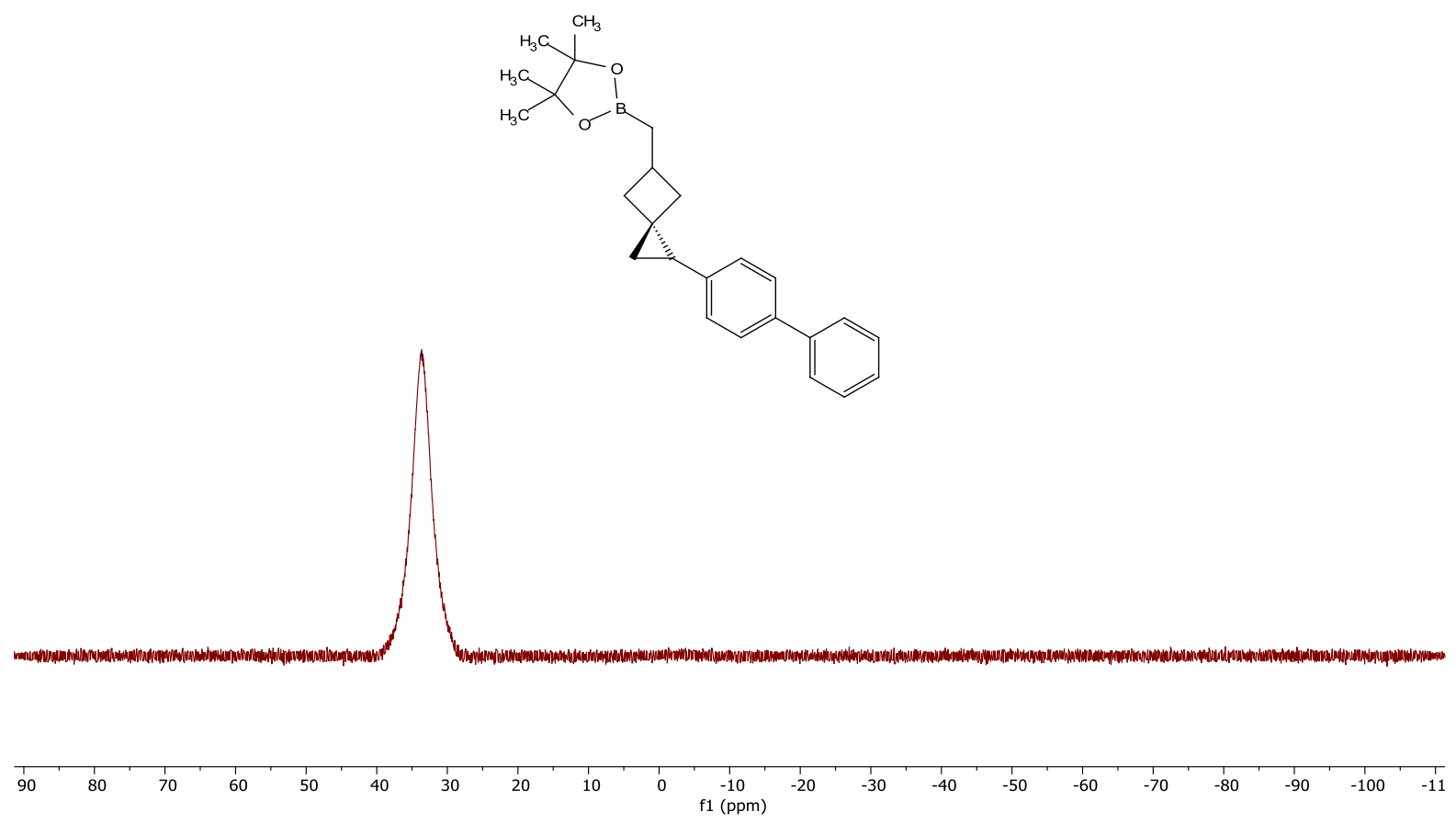

S113 
${ }^{1} \mathrm{H}$ NMR-16

46912 yu444.10.fid

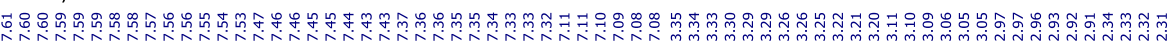

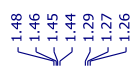

$\| / 4$
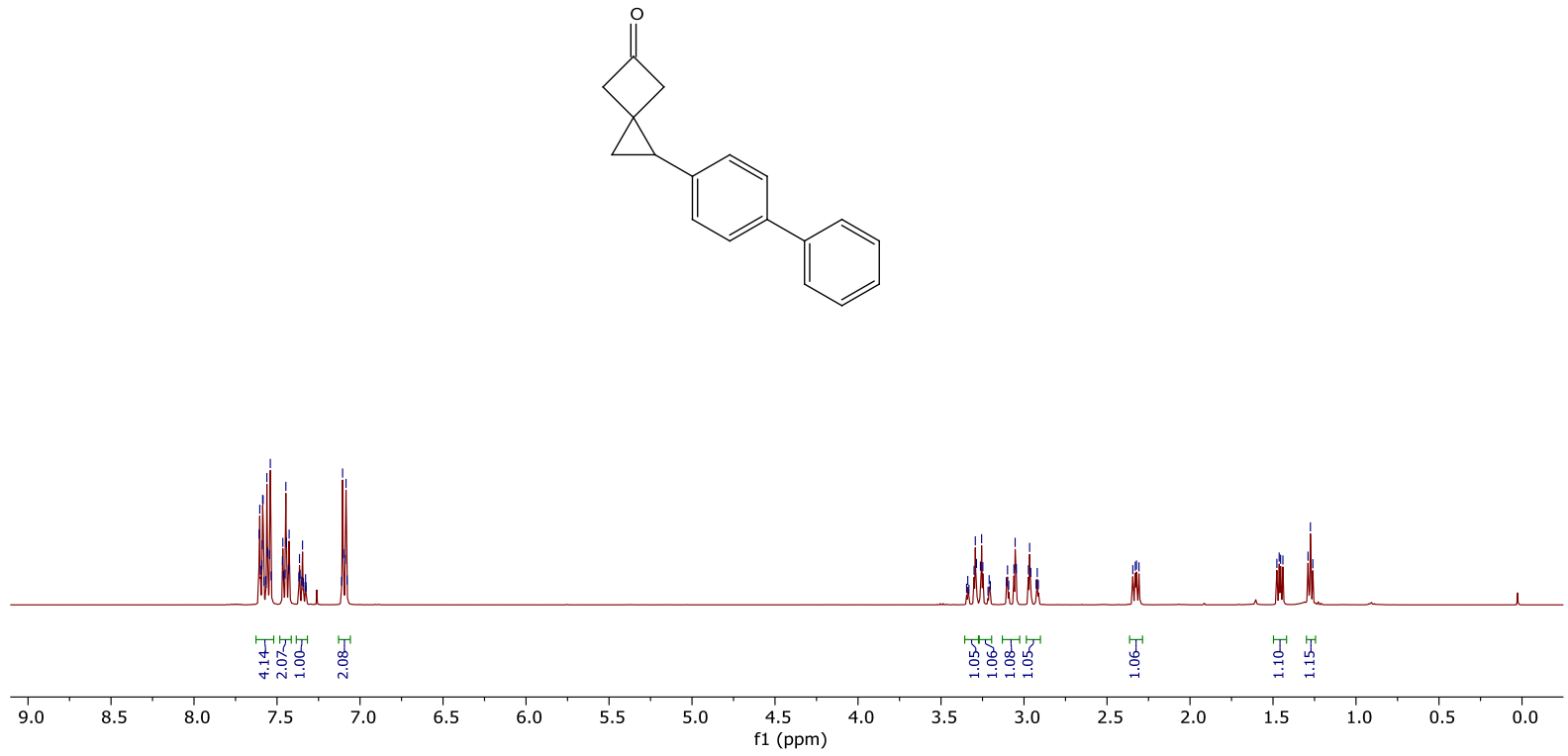

${ }^{13} \mathrm{C}$ NMR-16

46912 yu444.11.fid

لح

|

I
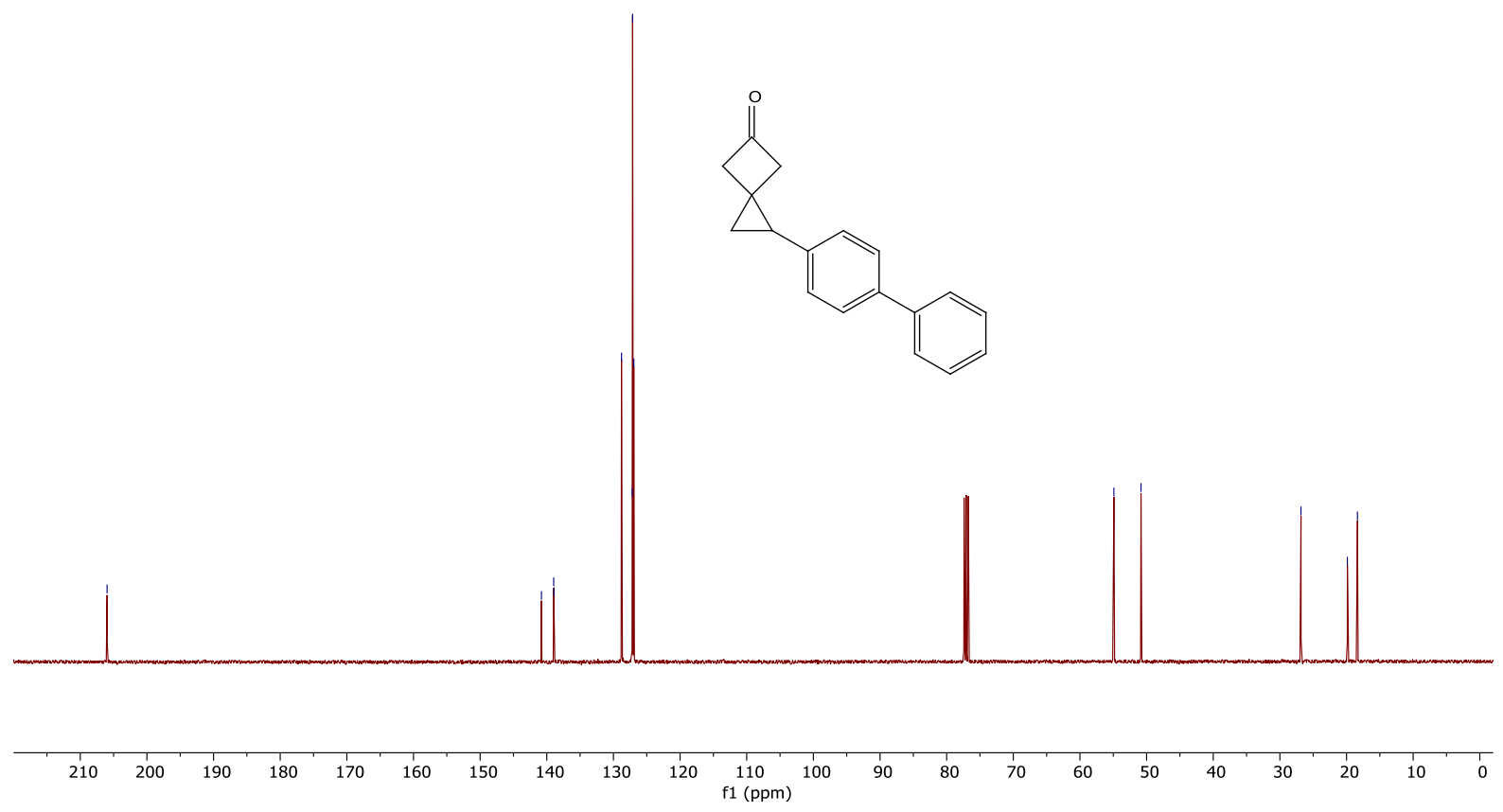

S114 


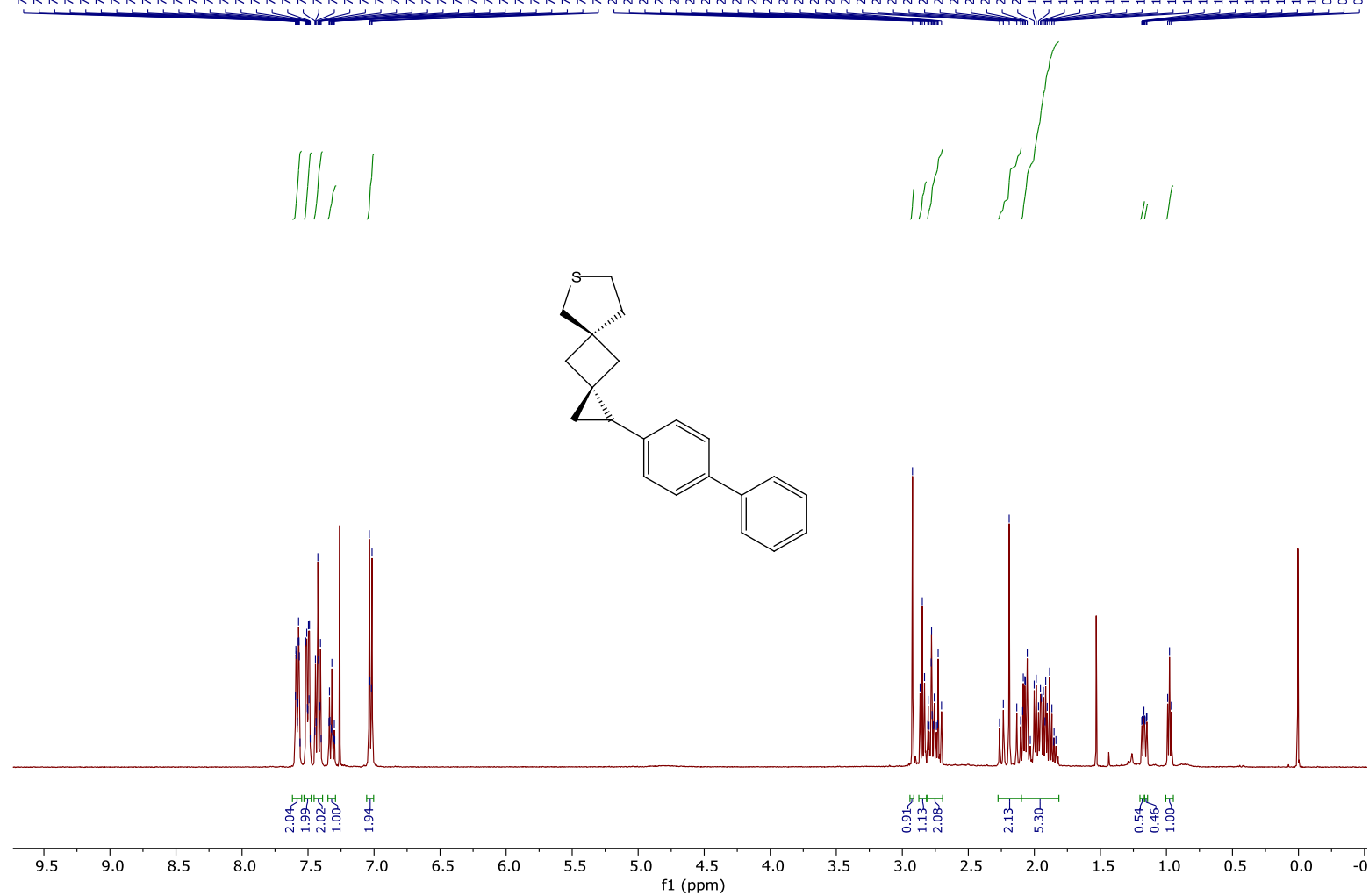

${ }^{13} \mathrm{C}$ NMR-17

45761 yu411-2.11.fid

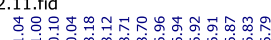

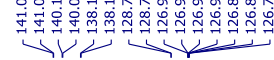

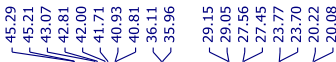
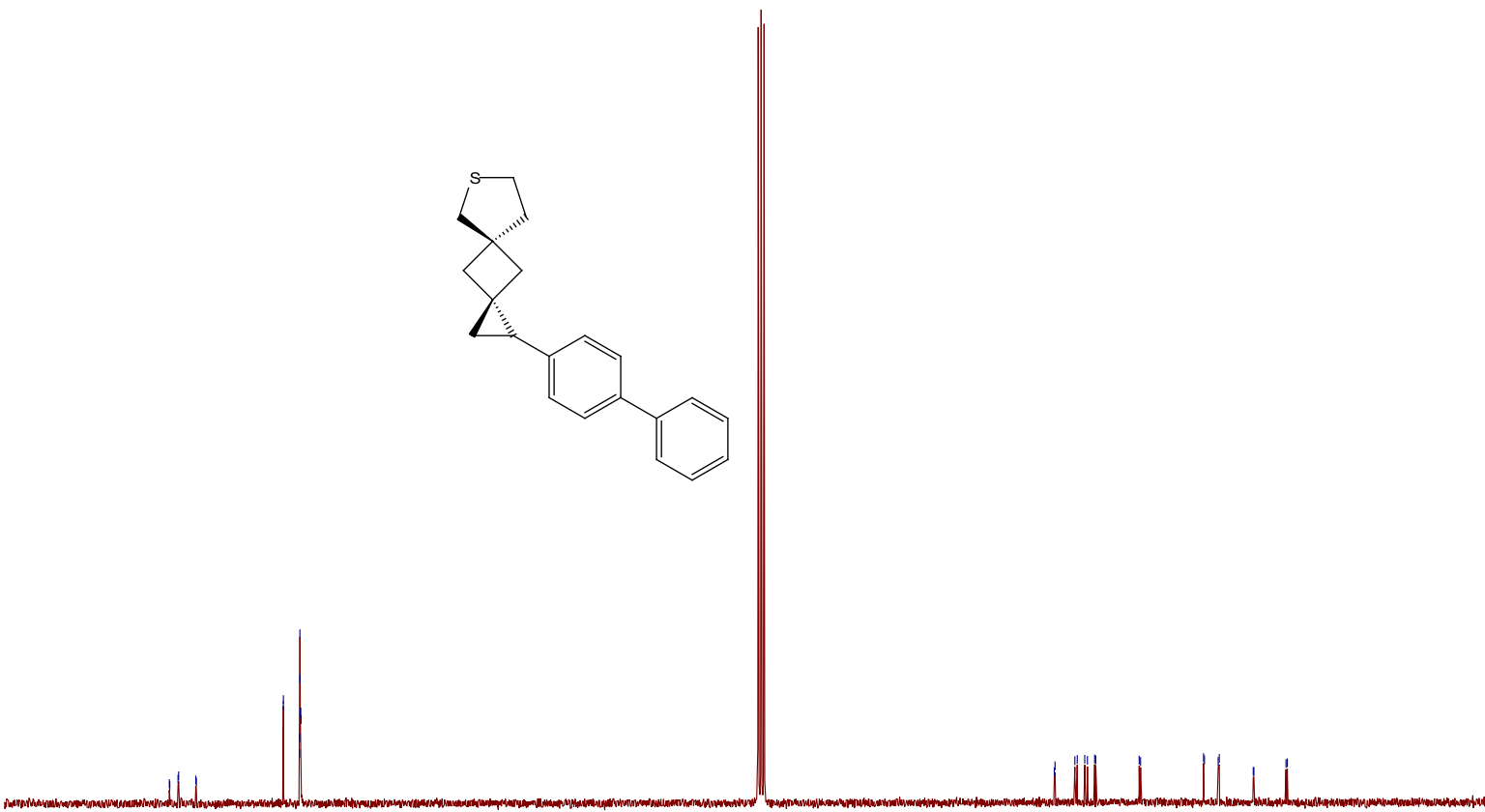

$120 \quad 110$

100

90

80
$f 1(p p m)$

$70 \quad 60$

$\begin{array}{llllll}1 & 1 & 1 & 1\end{array}$ 
${ }^{1} \mathrm{H}$ NMR-18

46630 yu437.10.fid

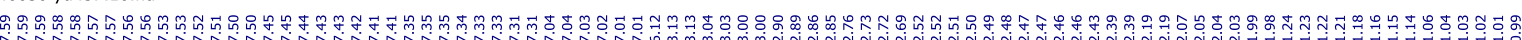
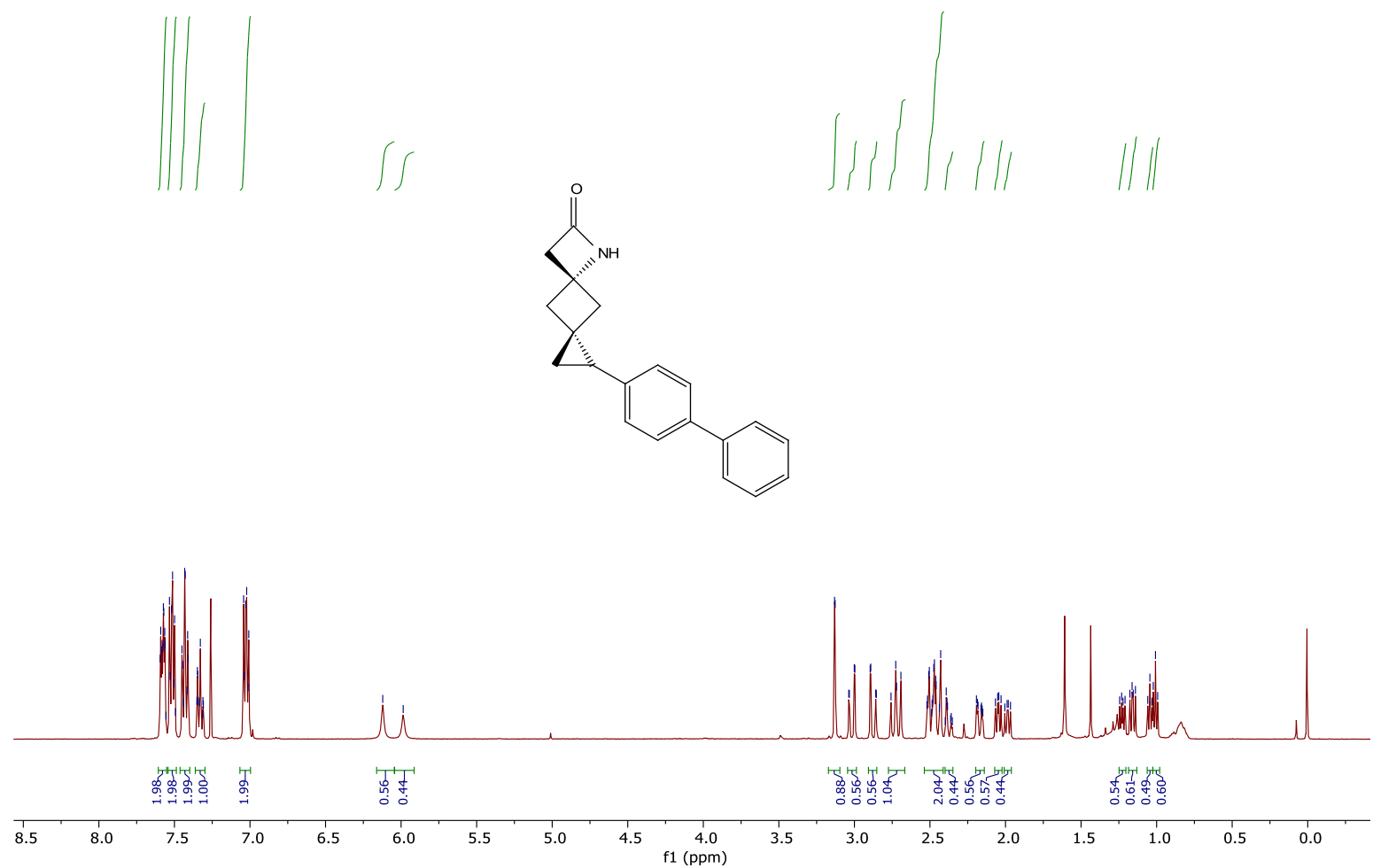

${ }^{13} \mathrm{C}$ NMR-18

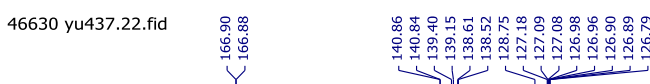

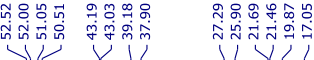
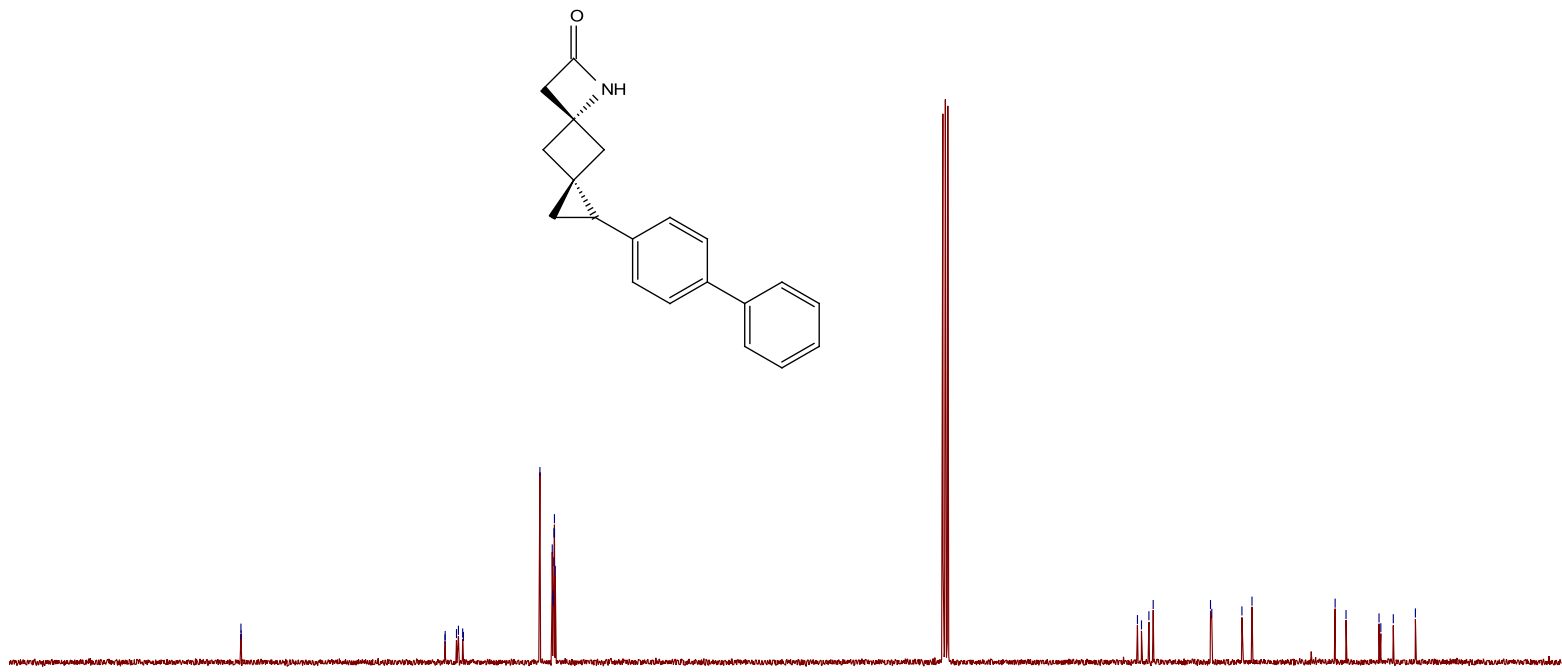

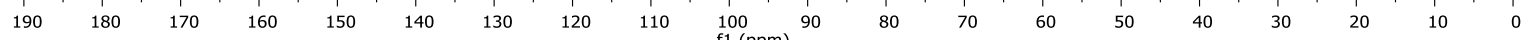


55457 yu594-1.10.fid

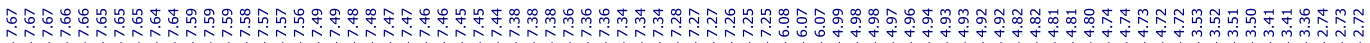

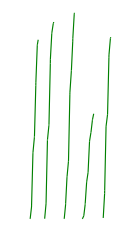<smiles>C#CC=C</smiles>
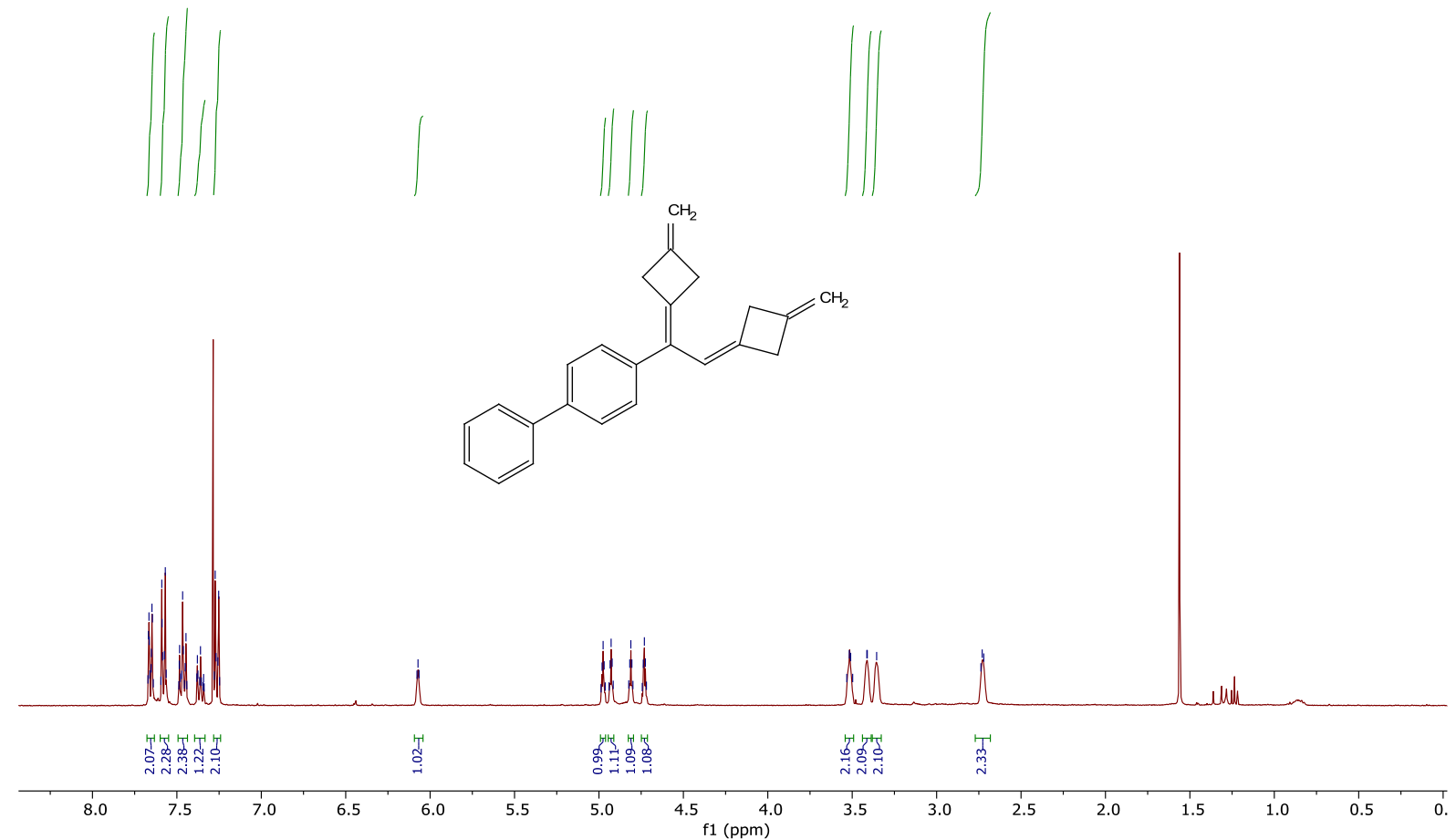

${ }^{13} \mathrm{C}$ NMR-10

55457 yu594-1.11.fid

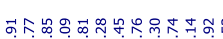

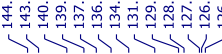

I)
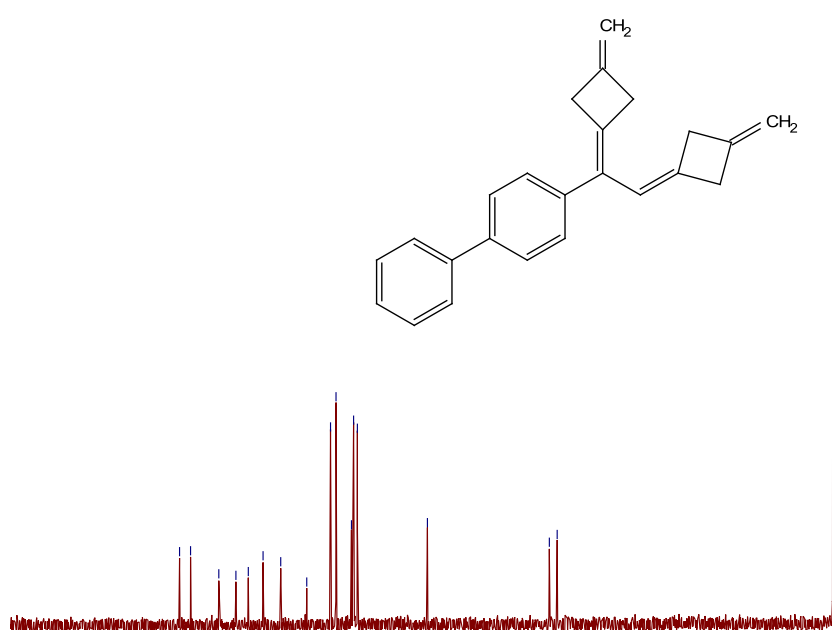